\title{
Application Protocol
}

Initial Graphics Exchange Specification (IGES)

RECEIVED

OCT 241934

OSTI

\section{Layered Electrical Product}
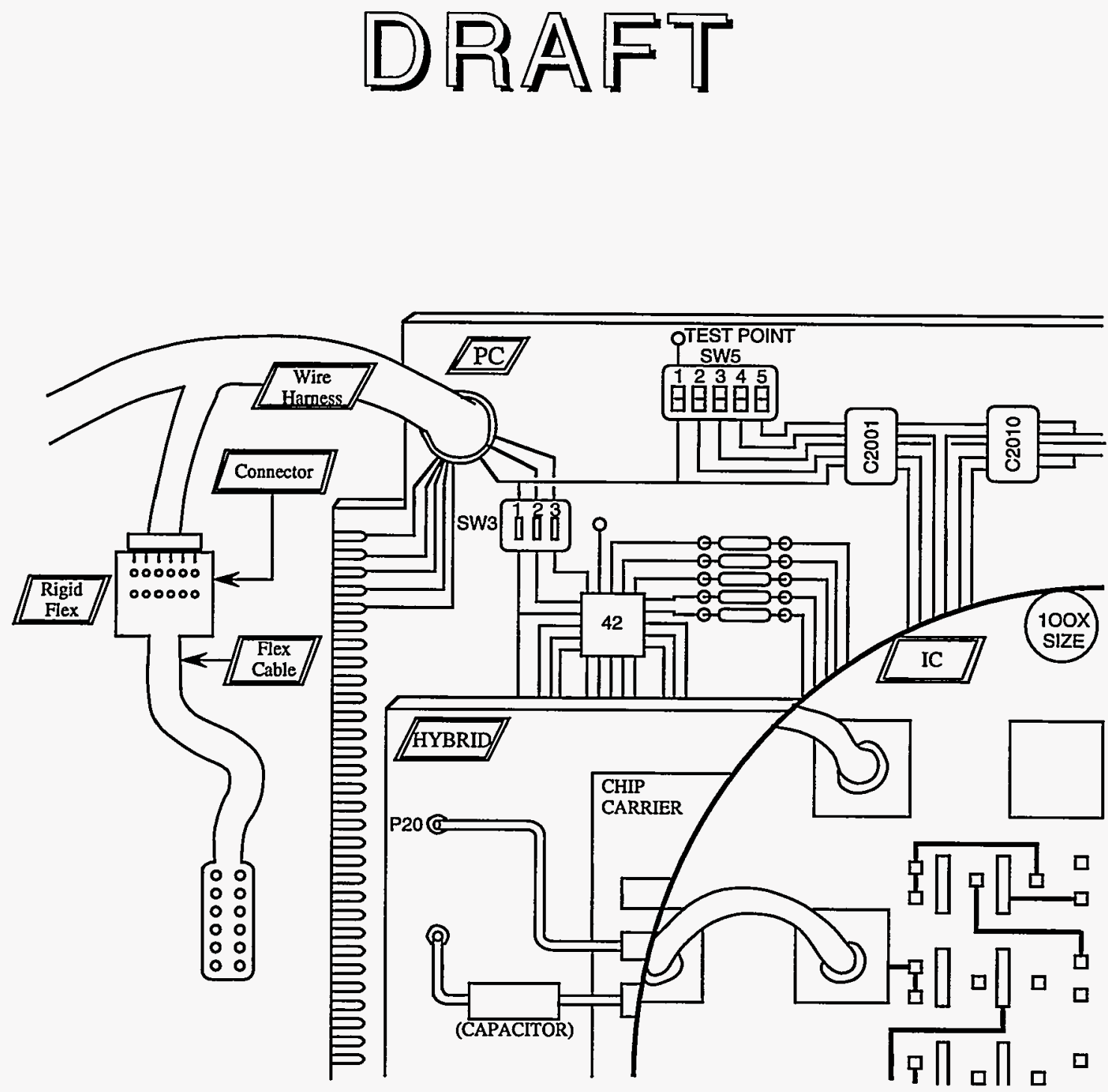

This work was supported by the United

States Department of Energy under

Conlract DE-AC?4-94AL\$5000. 


\section{DISCLAIMER}

This report was prepared as an account of work sponsored by an agency of the United States Government. Neither the United States Government nor any agency thereof, nor any of their employees, make any warranty, express or implied, or assumes any legal liability or responsibility for the accuracy, completeness, or usefulness of any information, apparatus, product, or process disclosed, or represents that its use would not infringe privately owned rights. Reference herein to any specific commercial product, process, or service by trade name, trademark, manufacturer, or otherwise does not necessarily constitute or imply its endorsement, recommendation, or favoring by the United States Government or any agency thereof. The views and opinions of authors expressed herein do not necessarily state or reflect those of the United States Government or any agency thereof. 


\section{DISCLAIMER}

Portions of this document may be illegible in electronic image products. Images are produced from the best available original document. 


\title{
"Information is neither Matter nor Energy"
}

Norbert Wiener

Initial Graphics Exchange Specification (IGES)

\section{LAYERED ELECTRICAL PRODUCT APPLICATION PROTOCOL (AP)}

\begin{abstract}
VERSION 1.0: This document is the IGES Electrical Applications Committee release of a complete application protocol for layered electrical products technology. Included is the collected set of design objects which the committee recommends for transfer of electronic product models and which are within the IGES domains of logical (e.g., schematic) and physical product drawing and computer presentation, data supporting manufacturing, and product testing. A broad range of electrical and electro-mechanical product models may be exchanged when this AP is applied together with the general product model exchange capabilities of IGES. The Committee recommends this AP version for translator development and encourages comments on the quality of the documentation and in particular the set of design data objects.
\end{abstract}

\begin{abstract}
An application protocol is an information systems engineering view of a specific product. The view represents an agreement on the generic activities needed to design and fabricate the product, the agreement on the information needed to support those activities, and the specific constructs of a product data standard for use in transfering some or all of the information required.

This applications protocol describes the data for electrical and electronic products in terms of a product description standard called the Initial Graphics Exchange Specification (IGES). More specifically, the Layered Electrical Product IGES Application Protocol (AP) specifies the mechanisms for defining and exchanging computer-models and their associated data for those products which have been designed in two dimensional geometry so as to be produced as a series of layers in IGES format. The AP defines the appropriateness of the data items for describing the geometry of the various parts of a product (shape and location), the connectivity, and the processing and material characteristics. Excluded is the behavioral requirements which the product was intended to satisfy, except as those requirements have been recorded as design rules or product testing requirements.
\end{abstract}




\section{PREFACE}

The Electricity Division of the Electronics and Electrical Engineering Laboratory and the Automated Manufacturing Research Facility of the Center for Manufacturing Engineering at the National Institute of Standards and Technology (NIST) completed a program in 1992 entitled "A Data Format Specification for Hybrid Microcircuit Assemblies." This project was sponsored by the Office of the Assistant Secretary of the Navy for Manufacturing Technology and was managed by the Naval Command, Control \& Ocean Surveillance Center, Research Development Test \& Evaluation Division (NCCOSC, RDT\&E), San Diego, Contract No. N0001991IPAKC1R, MOD/ AMEND No. 0A-001. The project objective was the development of a specification for a neutral format to promote the exchange of design and manufacturing data for hybrid microcircuit products. The resulting document "Initial Grapnics Exchange Specification (IGES) Hybrid Microcircuit Application Protocol," NIST TN 1295, January 1993 was distributed to Navy Contracting Officers, other program reviewers, and the Electrical Applications Committee (EAC) of the IGES Organization.

Reviews were held with industry, EAC, and the IGES Project from the Navy Program inception and throughout 1993. The discussions brought out the applicability of the Navy Hybrid Microcircuit work to a wide range of electrical product types. Following discussions in the EAC and with the Continuous Acquisition and Life Cycle Support (CALS) Interest Group, the editors of this document were tasked with bringing together the contributions of other authors and the NIST document contents mentioned above, resolving comments and experiences submitted during the years time.

The scope of this AP, formally stated in Section 1.2.3, includes the various aspects of all layered electrical products; the specification control drawings, circuitry and parts layout, and information concerning their fabrication. The specified data model is sufficiently detailed to support the design release, fabrication, and final assembly of a layered product system.

IGES is designed to support a broad range of applications and information, and it is recognized that few implementations will support all of the specification. An application protocol defines a logical subschema of the IGES specification, the usage of the subschema, and the necessary benchmarks for testing implementations. The application protocol for layered devices builds on the previous work of the Electrical Applications Committee of the IGES/PDES Organization. Such documents are seen as important for the development of STEP (Standard for the Exchange of Product Model Data) application protocols.

The data structures defined in this document are proposed in order to greatly improve the fidelity of layered electrical product applications data exchanged. The lack of fidelity has been termed "flavorings" which occur from two primary sources. The first flavoring source is attributed to inconsistent use of computer aided design (CAD) systems, often because of differing ways to describe the product within different organizations. The second source of flavorings often results when the data structures of different CAD systems are converted to or from IGES data entities. Where an identified subset of the IGES entities did not address the flavoring issue, this document specifies product objects which constrain both sources of flavorings. This AP is proposed as a replacement of MLL-D-28000 $\mathrm{A}^{2}$ Class 3. This revision will define a new MUL-D-28000 class for the transfer of layered electrical product data by use of future versions of IGES.

\footnotetext{
1. The Sandia National Laboratories on behalf of the Department of Energy contributed a great deal of information on the flavorings problem and possible solutions during the mid 1980s.
} 
The members of the EAC also note that other data formats may be employed. They would encourage continuation of work to achieve an industry consensus of the activity and reference model sections (Appendix A and 4.2 respectively) and additional interpretation (sec. 5) models as needed for each additional format supported. Comments on this document should be sent to:

Sandia National Laboratories

Larry O'Connell

Mail Stop 0955

P. O. Box 5800

Albuquerque, NM 87185-0955

(301) 844-1061

E-Mail: ljoconn@sandia.gov

\section{Future Directions}

The Application Activity Model (Appendix A) and Application Reference Model (sec. 4.2) contain sufficient detail to support design release, fabrication, and final assembly of electrical product system. The IGES models (sec. 5) in this document, however, are not meant to fully support product testing, analysis, and manufacturing. A full life-cycle data standard-possibly based on the Standard for the Exchange of Product Model Data (STEP)—will be necessary to support all phases of product processing.

This document does not complete the fabrication definition in terms of the sequence of processing steps and the parameters used in those steps.

This AP AIM is currently limited to layered electronic product (LEP) information contained in electronic computer-aided design (ECAD) systems. These systems are in widespread use. Their ability to translate data between dissimilar systems is a high priority in the user community. IGES serves as the implementation for this information because most ECAD suppliers are familiar with the specification and support it in their software packages.

A broadened capability for this AP that includes manufacturing, test, simulation, behavior, and documentation is desired by users and manufacturers of hybrids. Much of this information is outside the scope identified in of most ECAD systems and the IGES specification.

\section{About the Document}

The style, format, and much of the material for this application protocol was taken from the "3D Piping IGES Application Protocol." That AP specifies the mechanisms for defining and exchanging 3D piping system models in IGES format. ${ }^{3}$ The "3D Piping IGES Application Protocol" was the first IGES AP to be delivered to industry and led to the development of STEP (Standard for the Exchange of Product Model Data) application protocols. The authors of this document are indebted to Mark Palmer for his work and the guidance that it provides. It is the

2. MIL-D-28000 is the Department of Defense specification that identifies the requirements to be met when product definition data is delivered in IGES format.

3. See Section 2.2, Reference 16 (NOTE: This reference was the source of AP material, but is not the most current version of the IGES Piping AP.). 
authors' belief that if the CAD/CAM industry is to use application protocols consistently, we need consistent application protocols.

Preparation of this document is a tribute to the ability to move text and graphics between applications and computer systems. ${ }^{4}$ The text was originated in WordPerfect ${ }^{\mathrm{TM}}$ DOS $^{\mathrm{TM}}$ and filtered into FrameMaker ${ }^{\mathrm{TM}} 2.1$ on a Sun ${ }^{\mathrm{TM}}$ workstation for the original formatting and some of the graphics. The AAM model was developed in Design/IDEF ${ }^{\mathrm{TM}}$, and glossary in Hypercard; both on the Macintosh ${ }^{\mathrm{TM}}$. The ARM was drawn in MacDraw II ${ }^{\mathrm{TM}}$. The AIM was developed at International TechneGroup Inc. (ITI) where the text was written in Word ${ }^{\mathrm{TM}}$ for DOS and the graphics drawn in AutoCAD ${ }^{\mathrm{TM}}$. The graphics were transmitted to Raytheon in IGES format where they were edited and translated into FrameMaker ${ }^{\mathrm{TM}}$ MIF format. Final document assembly was done in FrameMaker ${ }^{\mathrm{TM}}$ 3.0/Mac and LASER-printed from Frame's PosTSCRIPT ${ }^{\mathrm{TM}}$ output.

\section{ACKNOWLEDGMENTS}

The editors thank the following individuals for contributions to the development of this document:

Authors and Contributors to the "IGES Hybrid Microcircuit Application Protocol":

Thomas Leedy - the author of the first work toward this document-and Curtis Parks, NIST; Roger McCollough and Charles Azu, NCCOSC; Larry Savage and Patrick Toomey, and Thomas Makoski, ITI, Inc. The product objects were developed by Diane Elmer, Scicards; Bernard Gilbert, Prime-ComputerVision; Dave Kehmeier, Mentor Graphics; Randy Lawson, Cadence Design Systems, Inc.; Thomas Makoski, ITI, Inc.; and Anthony Prince, Intergraph-Dazix.

Authors and Contributors to this Document:

Larry O'Connell (Chairman of the Electrical Applications Committee, IGES/PDES Organization), Sandia Laboratories; together with: Bill Loye, Loye \& Associates; Curtis Parks, National Institute of Standards \& Technology, John Russell, Honeywell Commercial Flight Systems; and the membership of the Electrical Applications Committee.

Special thanks to Gaye Garrison, PC Support Inc., for document editing and file preparations.

4. Word and DOS are trademarked by Microsoft Corporation; WordPerfect is trademarked by the WordPerfect Corporation; Framemaker is trademarked by Frame Technology Corporation; Sun is trademarked by Sun Microsystems, Incorporated; Design/IDEF is trademarked by Meta Software Corporation; Hypercard and Macintosh are trademarked by Apple Computer, Incorporated; MacDraw is trademarked by Claris Corporation; AutoCAD is trademarked by Autodesk, Incorporated; and PoSTSCRIPT is trademarked by Adobe Systems, Incorporated. Trademarked products were mentioned in this document as examples of the range of possible products and are not necessarily endorsed by the contributors to this document. 


\section{Table of Contents}

Abstract i

Preface ii

Future Directions iii

About the Document iii

Acknowledgements iv

\section{INTRODUCTION 1}

1.1. Purpose 1

1.2. Background 1

1.2.1. Electrical Assembly Complexity 2

1.2.2. Consistent Information for Concurrent Engineering 3

1.3. APPLICATION PROTOCOL CONTENTS 4

\section{APPLICATION AND CORE REQUIREMENTS 6}

2.1. Application Information Requirements 6

2.2. Application and ARM Correspondence 7

2.3. Normative References 9

2.4 Informative References 9 


\section{Contents}

\section{DEFINITIONS 13}

3.1. IGES Application Protocol Definitions 11

3.2. LEP Application Definitions 13

4. INFORMATION REQUIREMENTS AND APPLICATION REFERENCE MODEL 31

4.1 Application Data Views 31

4.2. Application Reference Model 31

4.2.1. Descriptions of the ARM Data Constructs 31

4.2.1.1. ARM Methods and Language 31

4.2.2. Data Construct Definitions 33

4.2.2.2. Data Construct Assertions 33

4.2.3. ARM Diagrams 34

\section{IGES APPLICATION INTERPRETED MODEL 43}

\subsection{ARM to AIM Mapping 44}

5.2. IGES Structure and Syntax of the Application Interpreted Model 44

5.2.1. IGES File Structure 44

5.2.1.1. START Section 45

5.2.1.2. TERMINATE Section 45

5.2.1.3. GLOBAL Section 45

5.2.1.4. DIRECTORY ENTRY and PARAMETER DATA Sections 45

5.2.2. Global Values and Entity List 45

5.2.3. Basic Syntax used in the AIM 50

5.2.3.1. Object Definition Block 50

5.2.3.2. Object Instance Block 51

5.2.3.3. Object Value Block 51

5.2.3.4. Object Reference Mechanism 52

\subsection{AIM Object Models 55}

5.3.1. Interface Object Models 55

5.3.1.1. LEP Part Library IGES File 55

5.3.1.2. LEP Physical Layout IGES File 56

5.3.1.3. LEP Technical Illustration IGES File 57

\subsubsection{LEP Property 59}

5.3.2.1. Component Placement Keepin/Keepout 60

5.3.2.2. Drawing Definition 61

5.3.2.3. Drawing Instance 62

5.3.2.4. Generic Part Definition 63

5.3.2.5. Generic Part Instance 64

5.3.2.6. Hole 65

5.3.2.7. Join 66

5.3.2.8. Jumper Wire 67

5.3.2.9. Keepin/Keepout 68

5.3.2.10. Land 69

5.3.2.11. Layer Outline 70

5.3.2.12. LEP Artwork Stackup Property (Type 406 Form 25) 71 
5.3.2.13. LEP Definition 72

5.3.2.14. LEP Instance 74

5.3.2.15. Level to LEP Layer Map Entity (Type 406 Form 24) 75

5.3.2.16. Net 76

5.3.2.17. Package Symbol Definition 78

5.3.2.18. Package Symbol Instance 80

5.3.2.19. Padstack Definition 81

5.3.2.20. Padstack Instance 82

5.3.2.21. Panel Definition 83

5.3.2.22. Panel Instance 84

5.3.2.23. Part Placement Boundary 85

5.3.2.24 Part Thermal Outline 86

5.3.2.25. Pin 87

5.3.2.26. Routing Keepin/Keepout 89

5.3.2.27. Test Point 90

5.3.2.28. Trace Keepout 91

5.3.2.29. Via 92

5.3.2.30. Via Keepin/Keepout 93

5.3.2.31. Wire Bond 94

\subsubsection{Display Geometry Object Models 95}

5.3.3.1. Arc 95

5.3.3.2. Circle 96

5.3.3.3. Closed Curve 97

5.3.3.4. Geometry Figure Definition 98

5.3.3.5. Geometry Figure Instance 99

5.3.3.6. Line Entity (Type 110, Form 0) 100

5.3.3.7. Multi-Line 101

5.3.3.8. Polygon 102

5.3.3.9. Point Entity (Type 116, Form 0) 103

5.3.2.10. Predefined Planar Shape 104

\subsubsection{Miscellaneous Object Models 105}

5.3.4.1. Generic Data Property (Type 406, Form 27) 106

5.3.4.2. Hierarchy Property (Type 406, Form 10) 111

5.3.4.3. Name Property (Type 406 Form 15) 112

5.3.4.4. Network Subfigure Definition Entity (Type 320, Form 0) 113

5.3.4.5. Network Subfigure Instance Entity (Type 420, Form 0) 114

5.3.4.6. Object Locator 115

5.3.4.7. Part Number Property (Type 406, Form 9) 116

5.3.4.8. Sectioned Area Fill Definition 117

5.3.4.9. Subfigure Definition Entity (Type 308, Form 0) 118

5.3.4.10. Subfigure Instance Enitiy (Type 408, Form 0) 119

5.3.4.18. Text String 120

\subsubsection{Semantic Item 121}

\subsubsection{DE Referenced Object Models 122}

5.3.6.1. Level Definition 123

5.3.6.2. Line Width Definition 124

5.3.6.5. Transformation Matrix 125

5.3.6.6. Transformation (Mirrored) Entity (Type 124, Form 1) 126

5.3.6.7. Transformation Matrix (Normal) Entity (Type 124, Form 0) 127

\subsubsection{DE Section Object Models 129}

5.3.7.1. Associativity DE 129

5.3.7.2. Definition DE 130

5.3.7.3. Geometry DE 131

5.3.7.4. Instance DE 132

5.3.7.5. Property DE 133 


\section{Implementation and Conformance Testing Guidelines 137}

6.1. PROCESSOR CONFORMANCE REQUIREMENTS 137

6.1.1. Testing Considerations 138

6.1.2. Conformance Considerations 138

6.2. Test Purposes and Test Groups 139

6.3. LEP Constructs 146

6.3.1. LEP Constructs Supporting Signal Conductivity 146

6.3.2. LEP Constructs Supporting a Physical Representation 147

6.3.3. LEP Constructs Supporting a Logical Representation 147

6.4. Entity Usage in a PDQ LEP Representation 147

6.4.1 DE Fields are Not Expected to Convey Meaning 148

6.4.2. Entities Which May Appear in a PDQ LEP Representation 149

6.4.2.1. LEP Usage of Geometry 149

6.4.2.2. LEP Usage of Annotation 150

6.4.2.3.LEP Usage of Existing Specific Property Entities 151

6.4.2.4. LEP Usage of the (Augmented) Generic Data Property Entity 152

6.4.3. Entities Which Shall NOT be Used in a PDQ LEP Representation 152

6.5. LEP Entity Usage for Core Applications 153

6.5.1. LEP Entity Usage for Padstack Representation 153

6.5.1.1. LEP Entity Usage for a Physical Padstack Definition 153

6.5.1.1.1. Entities Required to Add the Representation of Drilled Holes into a Padstack 154

6.5.1.1.2. Entities Required to Add Display Appearance of Holes in a Padstack 155

6.5.2. LEP Entity Usage for Physical Component Representation 157

6.5.2.1. LEP Entity Usage for Physical Component Definition 157

6.5.2.2. LEP Entity Usage for Physical Component Instance 158

6.5.2.3. LEP Usage for Physical Connect Point 160

6.5.2.4. LEP Entity Usage for Physical Via 160

6.5.3. LEP Entity Usage for Physical Mechanical Part Representation 162

6.5.4. LEP Entity Usage for Physical PWB Representation 163

6.5.4.1. LEP Physical PWB Definition 164

6.5.4.2. LEP Physical PWB Instance 165

6.5.4.3. LEP Physical Flow Associativity (Signal/Netlist) 166

6.5.4.4. LEP Physical LEP Signal Carriers (Traces, Shapes) 167

6.5.4.5. LEP Physical PWB Features: Fiducials 168

6.6. LEP Entity Usage for Specific Applications 169

6.6.1. LEP Entity Usage for Design/Engineering Applications 169

6.6.1.1. LEP Entity Usage for Netlist Reports 169

6.6.1.2. LEP Entity Usage for Component Placement Reports 170

6.6.1.3. LEP Entity Usage for Bill of Materials Reports 171

6.6.2. LEP Entity Usage for Manufacturing Applications 173

6.6.2.1. LEP Entity Usage for Photo-lithography Tools 173

6.6.2.2. LEP Entity Usage for LEP Test Aids 174

6.6.2.3. LEP Entity Usage for Numerically Controlled (NC) Production Files 175

6.6.2.3.1. LEP Entity Usage for Component Insertion 176

6.6.2.3.2. LEP Entity Usage for Component Pick and Place 178

6.6.2.3.3. LEP Entity Usage for Component Verification 180

6.6.2.3.4. LEP Enitity Usage for Wire Wrap 181

6.6.2.3.5. LEP Entity Usage for Labelmaker 182

6.6.2.4. LEP Entity Usage for Panelization 182

6.6.3. LEP Entity Usage for Drafting/Documentation Applications 183 
6.6.3.1. Major Differences Between the Documentation and the PDQ Model of a Conformant LEP AP 183

6.6.3.2. Changes and Additions to the PDQ LEP AP 183

6.6.3.3. LEP Entity Usage for Fabrication Documentation 184

6.6.3.4. LEP Entity Usage for Assembly Documentation 184

6.6.3.5. LEP Entity Usage for LEP Technical Illustrations 185

6.6.4. LEP Usage for CAD to CAD Exchange 185

6.6.4.1. LEP Entity Usage for Layer Assignment 185

6.6.4.2. LEP Entity Usage for Color 185

6.6.4.3. Semantic Properties for LEP Applications 185

6.6.4.4. Entity Usage for Presentation (Appearance) 193

\section{A. Activity Model Contents A-1}

B. Application Protocol Usage Guide B-1

C. Technical Discussions C-1 


\section{List of Figures \& Diagrams}

4. ARM Figures

4-1. Component Parts of an IDEF Relationship 32

4-2. Cardinality 33

4-3. The Categorization Structure 34

4. ARM Diagrams

View 4-1 Top-Level Entities 36

View 4-2 Expanded Dependents of Pattern 37

View 4-3 Expanded Dependents of Hybrid Version 38

View 4-4 Expanded Dependents of Hybrid Assembly Occurrence 39

View 4-5 Expanded Component Model 40

View 4-6 Expanded Dependents of Assembly Consumables and Deposition Component 41

\section{AIM Figures (Note: AIM Objects are listed in Table of Contents)}

5-1. IGES Object and Referencing 50

5-2. References to Previously Defined Object 51

5-3. Object Field Value Restrictions 52

5.-4. Reference Mechanism 53

5-5. Representation Mechanism 53

\section{Implementation and Conformance Testing Figures}

6-1. LEP Information Interface Requirements 139

6-2. General Structure of Objects Found in a Padstack 156 


\section{List of Tables}

2-1. Application to ARM Entity Correspondence 8

6-1. Overview of DE Settings for PDQ 148

6-2. DE Settings of Annotation Entities in a PDQ IGES File 151

6-3. IGES Data Fragments Showing Padstack Structures 156

6-4. DE Settings of Via Entities in a PDQ IGES File 161

6-5. DE Settings of LEP Instance Entities in a PDQ IGES File 166

6-6. Level Function Properties (Type 406, Form3) 186

6-7. Generic Properties of Top Level LEP 187

6-8. Generic Properties of Network 188

6-9. Generic Properties of Substrates 188

6-10. Properties of Component and/or LEP 188

6-11. Generic Properties of Component Definitions (Type 308 or 320) 189

6-12. Generic Properties of Component Instances (Type 408 or 420) 189

6-13. Generic Properties of Connect Point (Type 132) 190

6-14. Generic Properties of Closed Curves 190

6-15. Generic Properties of Open Curves 191

6-16. Generic Properties of Feature Definitions 191

6-17. Generic Properties of Vias 191

6-18. Production Engineering Generic Properties 192

6-19. Insertion Machinery Generic Properties 192

6-20. Placement Machinery Generic Properties 192

6-21. Wire Wrap Application Properties 193

6-22. Production Test Generic Properties 193 


\section{BLANK PAGE}




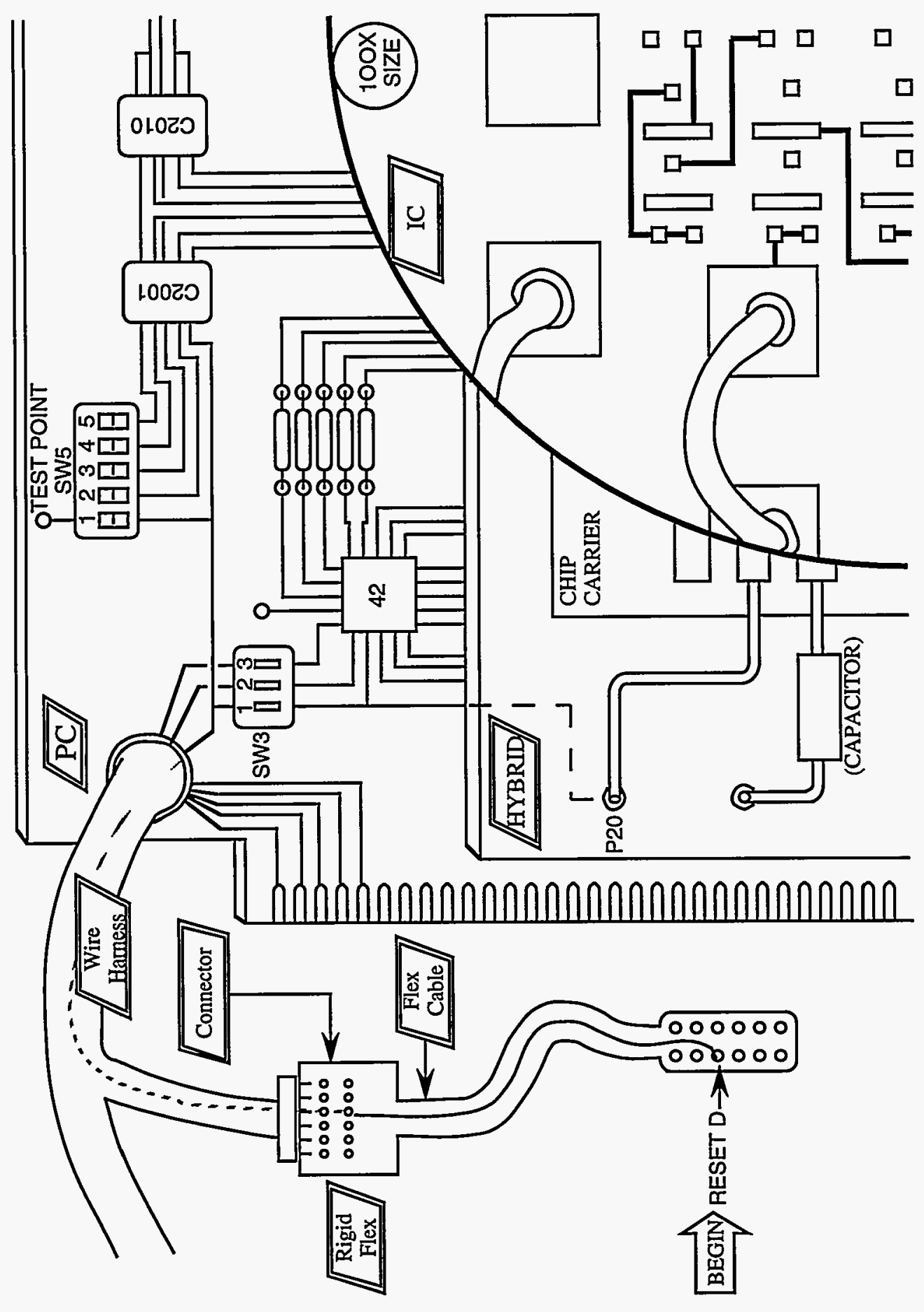

The illustration above has been included from the "Guide to IGES Electrical Entities," June 24, 1989 (Figure 1-2) which indicated that signal "Reset D" was involved with various technologies. Each of these technologies are considered within the scope of this document. 


\section{Initial Graphics Exchange Specification (IGES) LAYERED ELECTRICAL PRODUCT APPLICATION PROTOCOL}

\section{INTRODUCTION}

\subsection{Purpose}

This application protocol (AP) for layered electrical product (LEP) assemblies and parts to be incorporated onto such assemblies specifies the structure of Initial Graphics Exchange Specification (IGES) data for the representation of the product definition and for the exchange of these definitions from one LEP defining application to another. Since the LEP application protocol makes use of a specific interpretation of entities in the IGES file, both the sending and receiving processors must conform to this AP with regard to the information they process. It will not suffice to simply use IGES entities listed in a subset in the AP or in IGES or other documents. Proper packaging of connectivity information, for example, is crucial to success of some subsequent operations.

\subsection{Background}

The layered electrical product is a complex assembly made up of both graphic and non-graphic information, interelated through parent/child relations and associations.

LEPs are defined to be modules or subcircuits that are incorporated into larger electronic assemblies, in a hierarchy of devices for use in operational systems. The LEP may be either a monolithic device, such as an integrated circuit, or an assembly such as a hybrid microcircuit or printed circuit, or more than one assembly connected by means of a cable. Typically, an LEP is connected to the larger assembly with external leads or pins. An LEP assembly incorporates an insulating substrate onto which a mix of integrated circuits and other electronic components (such as thick- and thin-film devices) are interconnected.

A neutral data format serves several purposes: It permits the interchange of data between computer-aided design (CAD) and computer-aided manufacturing (CAM) systems. It allows the archiving of the LEP design in a format that can be used in the future, even if the original CAD or CAM systems or their software are no longer in use.

There are a number of motivations for developing a specified representation for LEPs. The most compelling motive is to reduce the errors created by different interpretations of a format as used for a particular product. A specified representation for LEPs can also minimize cost and maximize efficiency in the design and maintenance of translators. These uniform applications can provide means for coping with the increasing complexity of LEPs. In current practice, there is often the need for manual intervention in order to transfer the data between the CAD workstations and to the CAM stations that produce the LEP. Even in automated systems, the problem is compounded by the fact that a pair of data "translators" must be written for each pair of machines that must exchange data. If there are $n$ machines in the system-potentially $n *(n-1)$-translators are needed. 
Introduction

\section{Data hub concept}

A neutral format serves as a data "hub." Each station served must only translate data from its own format to the neutral format of the hub to be able to interchange with any other station interfacing with the hub. For example, data could be exported from a CAD workstation to the hub and be available there in neutral format for extraction by other CAD workstations and CAM machines. In this arrangement, for $\mathrm{n}$ machines in the system, there are potentially $2 *_{n}$ translators needed, this is a substantial reduction if $\mathrm{n}$ is greater than four machines.

\section{LEP Constructs Supporting Intelligent Representation}

Intelligent Representation of a LEP includes the ability to uniquely indentify different objects and attributes in the LEP. Subsequent applications may need to get "Product Data by Queries." Rather than refer to files and representations as "intellegent" we shall call them PDQ files or PDQ representations. For example, the ability to select the component by its reference designator of "R1" and its pin name of " 1 ". This will be critical for all automated shop floor processes, such as inserters, testers, pick/place, inspection, and others.

Automated assembly graphics designed to facilitate production build need such PDQ intelligence. Nearly any automated (or partially automated) process needs the ability to query signals, Components, Pins, Pads, Test Points, Vias, fiducials, tooling holes, and mounting hardware. PDQ intelligence also relates to the organization of the semantic information in the file. Semantic properties indicate the context of the data. The LEP is conceptually the same as the "model" in a mechanical system. It reflects a real world physical structure, with an unscaled coordinate system, and reference points to align the product and various manufacturing with inspection equipment.

\subsubsection{Electrical Assembly Complexity}

Assemblies have become increasingly complex in nature. One measure of this increased complexity is the ratio of the area of active elements (usually silicon chips) to the unoccupied area of the substrate of a hybrid microcircuit. In a single-chip package of a monolithic integrated circuit, the ratio is about 1:20; in today's typical hybrid, the ratio is from 1:10 to 1:6. Present engineering efforts have the goal of raising this ratio to $1: 1$, i.e., $50 \%$ of the substrate would be covered by silicon chips. These high-density hybrids are often referred to as multichip modules, or MCMs. To cope with this expanding complexity, hybrid manufacturing will increasingly depend on $\mathrm{CAD}$ and CAM techniques. For practical implementation of combined CAD and CAM techniques, it is important to have a single electronic representation of the CAD data available for interfacing with CAM environments. The representations described in this AP apply to MCM and conventional hybrid technology plus integrated circuits and printed boards and related products. The technologies of integrated circuits, printed wiring assemblies, flex cables and flex circuits have comparable density increases.

Many assembly manufacturers still use extensive paper documentation, such as prints and drawings, to document their product during manufacturing. Often, these drawings are produced on $\mathrm{CAD}$ stations that contain information that is not represented on the drawing and yet may be useful during the design process. As automated manufacturing methods become more available, such "paper" documentation will impede manufacturing. Further, as the complexity of electrical products increases, it will become much more necessary to convey this information to manufacturing machines in computer-comprehensible form. 


\subsubsection{Consistent Information for Concurrent Engineering}

Another benefit from a unified representation of the data describing a product is the ability to achieve concurrent engineering, which in the case of an LEP permits various automated and human resources to be applied to the design simultaneously. Since these resources share common data regarding the design, it is possible for various groups of engineers to refine the mechanical, electrical, thermal, and testability characteristics of the LEP in a much shorter time than would be required otherwise. In addition, concurrent engineering permits different application specialists to work in parallel with the designer. Thus, for example, those that are responsible for the manufacturing, assembly, quality, and reliability of an LEP design are able to provide suggestions concerning the design from its inception.

This method of business is in sharp contrast to the traditional methods where each department contributed sequentially to the design process of an LEP. Concurrent engineering methods promote a combined effort where all information builds on an existing model and changes can be easily accommodated through the separate functional areas. Since changes in the design are incorporated early in the design cycle, the costs of such changes is decreased. The result of effective concurrent engineering is a product at lower cost and with a shorter design cycle than would be realizable with traditional methods. Increased product quality results from accurate data transfer, as opposed to manual regeneration of CAD data on succeeding systems.

One of the ways in which the new method contrasts with the traditional methods is in the use of an architecture patterned after the ANSI-SPARC Three-Schema ${ }^{4}$ structures. In this architecture, a "conceptual schema" is created which functions as a means for managing the coupling between the "user views" of the information (the external schema), and the "database implementation" needed (the internal schema) to support the information system. The language reference manual for a transfer format standard usually describes the internal format, and within the descriptions the internal schema - to varying degrees of formality - may be found. The AP provides the external schema (herein called the Application Reference Model or ARM) for a particular application area and the way the data structures (herein called the Application Interpreted Model or AIM) are to be built from the primitive constructs of the standard. In this case, as in many others, the conceptual schema is not provided explicitly. Lacking an explicit conceptual schema, the mappings between the ARM and AIM are subject to interpretation.

There are several existing neutral file specifications to describe electrical and electronic functions. These include specifications developed by the Institute for Interconnecting and Packaging Electronic Circuits (IPC), the Electronic Design Interchange Format (EDIF), the Initial Graphics Exchange Specifications (IGES), and the VHSIC Hardware Description Language (VHDL). These neutral file specifications may support many of the data elements needed to represent an LEP design. Adding these formats is encouraged, and may be accomplished by adding the appropriate models to section 5 of this AP. During such additions, the remaining sections should be refined by agreement between the organizations that are responsible for the formats. Conflicting information requirements (e.g., sections 3 and 4 of this document) among different formats are not considered appropriate to the goals of product data consistency.

\footnotetext{
4. For more information on these data architectures see Reference 15.
} 


\subsection{APPLICATION PROTOCOL CONTENTS}

Section 2 of the AP provides a list of documents which are used in the construction of this protocol. Definitions of terms used in the AP are listed in alphabetical order in section 3. Part 3.1 of this section lists terms contained in the text of the AP while part 3.2 has terms contained in the various models (AAM, ARM, AIM) of the AP. Terms in both sections are listed along with reference to the models in which they appear. Section 4 contains the ARM; an engineer's view of data about the product being designed. In section 5, the "IGES-exchangable" information of the ARM is defined as product model objects defined in terms of IGES entities. Section 6 defines the tests for conformance to the IGES and the object definitions as required for information uses.

The following typesetting conventions have been used to identify the particular model referred to by semantic terms in the text of this document:

$\begin{array}{ll}\text { Roman, Initial Cap (followed by Type, Form numbers) } & \text { IGES entities } \\ \text { Italic, Initial Cap } & \text { ARM entities } \\ \text { ITALIC, ALL CAP } & \text { AIM objects }\end{array}$

\subsubsection{Terms and Definitions}

In addition to the definitions listed in Section 3, there are many terms associated with electrical product technologies as discussed below.

Products to which this AP apply are also distinguished by many "technology" terms. In particular, some of the terms which are associated with integrated circuits include custom, application specific integrated circuit (ASIC), gate array, digital, analog, mixed, and monolithic microwave integrated circuit (MMIC). Some of the terms which are associated with hybrid microcircuits include multi-chip module (MCM), single-chip module (SCM), microstrip assembly, thin film circuit, thick film circuit, green tape design, and surface mount technology. Some of the terms which are associated with printed circuit assembly (PCA) include printed circuit board (PCB), printed wiring board (PWB) or assembly (PWA), Flexible Circuits, Flexible Cables, and microstrip board.

The characteristics which distinguish the above domain of product types are their physical product model defined by features on one or more strata. Some features of various strata may be associated with a signal or signal bundle and/or various electrical properties.

The AP-applicable product types may also include features common to mechanical product types. Such features may include base plates, milled pockets, routed edges, threads or threaded inserts, mounting brackets, and heat sinks.

The AP information is applicable to the various ways that parts and components are defined. One way is the (external) package, usually as defined in Joint Electron Device Engineering Council (JEDEC) specifications, or the "footprint" for such a package. Another way may be the depiction of wire bonds electrically connecting a silicon chip to the leads of the package. Yet another way is the depiction of the part assembled as a component on another LEP such as an MCM or a PCA.

\subsubsection{Scope}

Layered Electrical Products include Flexible (Flex) Cables, Flex Circuits, Printed Circuit Assemblies, (includes Printed Wiring Boards), Hybrid Microcircuit Assemblies, Multi-Chip Modules, and Integrated Circuit die. The unifying element common to all these LEP technologies is the se- 
ries of photomasks used in their manufacture. Practitioners ${ }^{5}$ of each technoloy use some jargon different from all or most of the others, but the concepts are remarkably similar.

The scope of this Application Protocol includes:

- a reduced set of two-dimensional geometry sufficient to describe physical features of Layered Electrical Products, deposited components, and incorporated parts;

- connectivity of Traces, Conductive Areas, wirebonds, and Vias built into the LEP plus Pins, Pads, and Sockets of components incorporated into the LEP;

- patterns of photoplots and masks used in fabricating the LEP substrate;

- data plus context to support automated and semi-automated fabrication (such as numerically controlled drilling, panel layout, and automatic part insertion), testing of some kinds (such as bare board and in-circuit), technical illustrations (including process pictures and maintenance manuals), and Engineering Drawings (including schematics, netlists, bills of material, assembly drawings and layouts). Notice that this AP is not sufficient for these activities (for instance, a drafting AP may be needed to control dimensions and other annotation) but this AP controls the provision of much of the technical information needed by the activities.

The scope of this Application Protocol does not include:

- circuit simulation;

- behavior analysis;

- finite element analysis;

- drafting of engineering drawings;

- production of full color ilustrations;

- control of process operations;

- selection of component parts;

- control of automated testing;

- vendor qualification; nor

- dispensing of consumable properties.

The AP may cover some of the activities within its scope in less detail than users might like. If so, such users are invited to send constructive suggestions to the editor of this AP.

5. The Application Reference Model in 4.2.3 below was developed largely by people working in the domain of Hybrid Microcircuits. Some of the people involved had worked on the Cal Poly model years earlier. That model was developed by a team of people experienced with Printed Boards and Integrated Circuits. 


\section{APPLICATION AND CORE REQUIREMENTS}

This AP applies to electrical designs which were originated in a computer program (herein called "ECAD" system) designed to capture electrical-specific information. (Technically, this term often refers to Electrical Computer Aided Engineering (CAE) systems as well as to Electrical Computer Aided Design (CAD) systems.) Some electrical designs, drawings, or descriptions may be originated on non-ECAD systems sometimes called MCAD systems; IGES files from these systems shall follow the guidelines established within the IGES Drafting Application Protocol. The following subsections provide guidelines for determining the content of those applications which depend on the electrical data (most importantly connectivity data) defined within ECAD systems.

This AP requires that non-ECAD systems either display the graphics associated with the Network Subfigure Instances and Connect Point IGES entities, or convert them to their internal version of IGES Subfigure Instance and Point entities. Non-ECAD systems are not required to write out IGES files with Network Subfigure Instances and Connect Point entities

\subsection{Application Information Requirements}

The core requirements were determined by examining a portion of the product life cycle. These life cycle tasks were:

- Physical Design Layout

- Manufacturing

- Visualization (for assembly, troubleshooting, etc.)

- Testing

- Logistic Support Documentation

A single LEP design file may be used as the source for several different kinds of processes. Each independent entity in the ARM may also constitute an application requirement. Further, the child entities of these entities may, together with their existence-dependent entities, be used in an application structure. For a view of the relationships between core requirements and the ARM, see table 1 . The symbol "+" indicates that all child entities apply as well.

Parts are often involved in a lead preparation step such as lead bending and tinning prior to their being assembled on an LEP. Transfer of part models which specify these intermediate life cycle steps are considered within the domain of this AP.

During these life cycle stages there are data added such as pin swapping and netlist back annotation. These data are added within the CAD or CAE system; the IGES output file following such $\mathrm{CAE}$ operations is differentiated by a different file name. Different fabrication facilities have different capabilities and pre-defined processes. Thus it may be premature for the IGES file to contain detailed information governing the fabrication processes prior to the selection of a facility and receipt of a committment at that facility. As concurrent engineering becomes a reality, this will be less of a concern because the fabrication planner will participate in the definition captured and then transfered using IGES.

This AP is not applicable to the electrical product life cycle phases preceding the capture of the structural design by way of a schematic drawing or netlist representation unless the behavior is presented as a graphic such as a waveform. Where product behavioral definition exists in a data format other than IGES, provisions will be defined for the association of behavior at a logical pin (or "port") with a schematic or physical pin defined within the IGES file. Such association is fre- 
quently achieved in CAE systems by matching on the assembly component's reference designator and pin number occurring in each data definition.

The information for all life cycle phases may originate in a computer aided design (CAD) system. Some information may only be computer-processed within a Computer Aided Engineering (CAE) system. Or, part of the information for any category may be created by manual - non$\mathrm{CAD} / \mathrm{CAE}$ - means. LEP information from any source must be constrained semantically for use in the within the AP, and further, the requirements for the IGES form defined herein is (syntactically) constrained by the constructs in the application interpretation model.

All functionality categories which have been originated in a CAD system are capable of being transmitted to another, or an intermediate $\mathrm{CAD}$ or CAE system. In this event, much of the information may be "exchanged" without distinction. When electrical-specific information has been added by a CAE system, such information may not be comprehensible by a CAD system. The $\mathrm{CAD}$ system receiving a CAE-created file is required to ignore (i.e., to not process) the connectivity and electrical property entities, but to process and display the physical product model.

All information originated from a CAE system shall be processed by another CAE system; whether through direct conversion into the CAE native data, or through display to the CAE operator in such a way that the information may accommodated by manual entry means.

For example, a design exchanged between $\mathrm{CAD}$ systems may adopt a level of information content that is greater than the Drawing. The receiving CAD system would benefit by "knowing" the properties and rules defined in the source system in addition to the product as a drawing graphic.

\section{LEP Entity Usage for Core Applications}

Section 5 defines which entities are required to support a specific application. For example, production test requires Network Subfigure Instances (representing components) and Connect Points (representing pins), in order to identify each testable point uniquely.

Entities may not always map directly between various applications. For example, within the photolithography application, some programs will accept complex geometry, text strings, tapered lines, and subfigure constructions. Other programs can understand no more than simple fixed width lines (no arcs), and a few predefined geometries called flashes.

There are two different ways of looking at this problem. One approach would have the LEP AP detail which entities may be found in conformant files, implying a method to process those entities into something the application would accept. The second approach would detail which entities will probably be accepted by the application. The LEP AP follows the first approach, and assumes there is a tool available to perform required tasks of simplifying and remapping entities from the LEP AP conformant file.

\subsection{Application and ARM Correspondence}

The LEP is defined in this AP by a combination of documents (called presentation) and CAD/ ECAD data (called representation). The CAD/ECAD data consists of the screen display data and the CAD/ECAD product model itself. The ARM reflects the data distinction. For example, the product may be defined through a drawing produced manually or on a CAD system, such as a Specification Control Drawing. CAD systems may be utilized in such a way as to produce a "paper document" intended for human interpretation. Information such as the electrical schematic and waveforms are frequently treated as such drawing data. Some applications include the CAD 
or ECAD display information such as the color with which a particular system-layer is displayed. Some applications are developed such that lists may be extracted (e.g., net lists, test probe locations, and drill lists) from the ECAD or CAE model of the product.

For purposes of this AP, the following table indicates appropriate applications and the entities in the ARM which are related. The symbol " + " indicates that all child entities apply as well.

Table 2-1: Application to ARM Entity Correspondence

\begin{tabular}{|c|c|c|}
\hline Application & $\begin{array}{l}\mathrm{CAD} / \\
\mathrm{CAE}\end{array}$ & ARM Entities \\
\hline Bill of Materials & both & Component, Assembly Consumables, Substrate \\
\hline Package & $\mathrm{CAE}$ & Device Package \\
\hline Visualization & CAE & LEP Documentation + \\
\hline Geometry & both & Geometry \\
\hline Artwork & both & Pattern + \\
\hline $\begin{array}{l}\text { Surface mount pick \& } \\
\text { place }\end{array}$ & both & Area+, Flashed Geometry, Via Hole, Probe Pad \\
\hline Panel & both & Panel MultiUp, LEP Assembly \\
\hline Test programs & CAE & LEP Assembly Component \\
\hline Wirewrap & $\mathrm{CAE}$ & Pin + \\
\hline Flex tape & CAE & Device Package \\
\hline $\begin{array}{l}\text { DITMCO (continuity } \\
\text { test) support }\end{array}$ & CAE & Test, Net \\
\hline Thru-hole auto-insert & $\mathrm{CAE}$ & Process Step, LEP Assembly Component \\
\hline $\begin{array}{l}\text { Semi-automatic inser- } \\
\text { tion }\end{array}$ & CAE & Process Step, LEP Assembly Component \\
\hline $\begin{array}{l}\text { Combination test pro- } \\
\text { grams \& fixtures }\end{array}$ & CAE & Tool, Test \\
\hline $\begin{array}{l}\text { SMT LEP producibil- } \\
\text { ity }\end{array}$ & CAE & Process Step \\
\hline Adhesive dispencing & both & Process Step \\
\hline In-work assy graphics & both & LEP CAD Presentation \\
\hline
\end{tabular}

The Application Reference Model (ARM) is explained and presented in section 4. The ARM is constructed in terms used within the electronic design and fabrication community (i.e., an external 
schema). For example Part and Component are used, and are given definitions appropriate to those found in engineering as related to the use of CAD systems and supporting libraries. The DEF1X modeling technique is used to develop the ARM and a brief tutorial on how to read the model is included.

The ARM was constructed to depict the complete layout-to-manufacturing of the LEP; primarily to be understandable by the engineer and planner. The AIM, described below and closely related to the IGES file structures, may not contain all ARM structures but will be more detailed. The ARM entities which are not defined as AIM objects are graphically marked in the ARM.

The Application Interpretive Model (AIM) is described and presented in section 5. The mapping of the ARM to AIM is explained. The objects declared in this section are associated to general and specific CAD data items (i.e., an internal schema). A description of the IGES file structure and the breakdown of its sections is given. A modeling syntax is used to develop the AIM and a brief tutorial on how to read the model is included.

Section 6 details implementation and conformance guidelines for this Application Protocol. Process conformance requirements and testing purposes are given. Appendix A contains the Activity Model which indicates the processes which utilize ARM information. Appendix B has a parameter listing of typical LEP processing parameters/variables and their values.

\subsection{Normative References}

This AP is specifically applicable to IGES version 5.2. The versions of IGES listed below include electrical product capability; any one of them may be cited in an exchange. Earlier versions of IGES may contain electrical product-defining objects compliant with this AP.

IGES versions 1.0, 3.0, 4.0, and 5.2 have been registered as an American National Standard (ANS) Y14.26M by the American National Standards Institute. Additionally, IGES version 4.0 is registered by NIST as a Federal Information Processing Standard; FIPS PUB 177. IGES electrical-related entities and the versions in which each was first published are identified in Section 5 of this document.

1. IPC-T-50E, "Terms and Definitions for Interconnecting and Packaging Electronic Circuits," February, 1991, Institute for Interconnecting and Packaging Electronic Circuits, Lincolnwood, IL 60646.

2. MIL-D-28000A, 10 February 1992, and Amendment 1, February 1993; Digital Representation for Communication of Product Data; IGES Application Subsets and IGES Application Protocols, Director, CALS Evaluation and Integration Office, Pentagon Room 3D833, Washington, DC 20301

3. MIL-H-38534, "Hybrid Microcircuits, General Specification for," 31 March 1989.

4. The Initial Graphics Exchange Specification (IGES) Version 5.2, an American National Standard, US PRO/IPO-100, November, 1993. Available from the National Computer Graphics Association (NCGA) Technical Services and Standards, 2722 Merilee Drive Suite 200, Fairfax, Virginia 22031.

\subsection{Informative References}

5. Smith, B., Wellington, J., The Initial Graphics Exchange Specification (IGES) Version 3.0, 
NBSIR 86-3359, U.S. National Bureau of Standards, April 1986. Available from the National Technical Information Service (NTIS), Fairfax, VA 22031 as PB86-199759.

6. Smith, B., Rinaudot, G., Wright, T.; Reed, K., The Initial Graphics Exchange Specification (IGES) Version 4.0, NBSIR 88-3813, U.S. National Bureau of Standards, June 1988. Available from the National Technical Information Service (NTIS), Fairfax, VA 22031 as PB88-235452.

7. Reed, K., Harrod Jr., D., Conroy, W., The Initial Graphics Exchange Specification (IGES) Version 5.0, NISTIR 4412, National Institute of Standards \& Technology, September 1990. Available from the National Computer Graphics Association (NCGA) Technical Services and Standards, 2722 Merilee Drive Suite 200, Fairfax, Virginia 22031.

8. Reed, K.; Kelly, J.C., Harrod Jr., D., Conroy, W., The Initial Graphics Exchange Specification (IGES) Version 5.1, US PRO/IPO, September, 1991. Available from the National Computer Graphics Association (NCGA) Technical Services and Standards, 2722 Merilee Drive Suite 200, Fairfax, Virginia 22031.

9. O'Connell, L., "Guide to the IGES Electrical Entities," EACP-2.3, unpublished, June 24, 1989, Available from IPO Office, National Institute of Standards and Technology, Bldg. 220, Room A-127, Gaithersburg, Maryland 20899

10. Harrison, R.J. and Palmer, M. E., "Guidelines for the Specification and Validation of IGES Application Protocols" NISTIR 88-3646, January 1989. Available from the National Technical Information Service (NTIS), Springfield, VA 22161, October 1990, (110 pages).

11. "Integration Definition for Information Modeling (IDEF1X)," FIPS PUB 184, National Institute of Standards and Technology, Computer Systems Laboratory, Gaithersburg, Maryland 20899, December 21, 1993.

12. "Integration Definition for Function Modeling (IDEF0)," FIPS PUB 183, National Institute of Standards and Technology, Computer Systems Laboratory, Gaithersburg, Maryland 20899, December 21, 1993.

13. Parks, C., "Tutorial: Reading and Reviewingthe Common Schema for Electrical Design and Analysis," Proceedings of the 24th Design Automation Conference; IEEE \& ACM, June 30, 1987, paper 27.2.

14. Loomis, M., "Data Modeling - The DEF1X Technique," Proceedings of the IEEE 1986 Phoenix Conference on Computers and Communications, Phoenix Arizona, page 146-149.

15. Tsichritzis, D.; Klug, A.; The ANSI/X3/SPARC DBMS Framework Report of the Study Group on Database Management Systems, X3 project 226, 1977, AFIPS Press, 210 Summit Avenue, Montvale, NJ 07645

16. Palmer, M.E. and Reed, K.A., "3D Piping IGES Application Protocol, Version 1.0," NISTIR 4420 (257 pages), October 1990, Available from the National Technical Information Service (NTIS), Springfield, VA 22161. 


\section{DEFINITIONS}

Section 3.1 contains those definitions which apply to the notion of an application protocol; they are included as an assist in understanding the content of this document. Section 3.2 contains the context definitions; for functions (in Appendix A), entities (in Section 4.2.3), and objects (in Section 5).

\subsection{IGES Application Protocol Definitions}

APPLICATION: An enterprise process that puts product data to use. The scope of an application is defined by the class of product, the supported stages in the life cycle of the product, the uses of the product data, and the disciplines that participate in that use.

APPLICATION ACTIVITY MODEL (AAM) - A structured decomposition of the tasks which are followed to achieve the particular product defined by the AP. The AAM identifies the context of the AP as well as the activities which create and use the information being defined. The AAM is constructed to be generally representative of the industry, and includes processes which are, or could be, largely automated.

APPLICATION INTERPRETED MODEL (AIM) - An information model that identifies the information structures of a particular data format specification. The AIM is defined to accomplish a physical implementation of an associated application reference model (ARM). This AIM is prepared at a level of abstraction that is sufficient for selecting the necessary entities for an application.

APPLICATION PROTOCOL (AP): Defines the scope and information domain of an application and specifies the rules for using IGES, or some other standard, to enable unambiguous transfer of the application information, and the testing of those transfer implementations.

APPLICATION REFERENCE MODEL (ARM) - An information model that describes the information structures and constraints for an application area. The ARM is defined in terms of Entities, Attributes, and Relationships. The information model uses application specific terminology and rules familiar to an expert from the application area. The model is independent of any physical implementation and can be validated by an expert from the application area.

APPLICATION SUBSET - An unambiguous set of entities which span the data requirements of the specified application. The set of entities is determined on the basis of the Application Reference Model.

ASSEMBLY - In general (e.g., from Websters): Parts fitted together to make a whole. The assembly referred to in this AP is taken to mean parts placed on, and/or connected to, an interconnecting part generally in the form of a flat substrate on which has been deposited alternate layers of metalized patterns and insulation material; the whole of which has been defined as to provide a specified part of the function of a system. An assembly may be used as a part on another assembly.

ATTRIBUTE - A property or element of information associated with an Entity. An instance of the attribute must exist for any instance of the Entity to which it belongs.

ENTITY - The basic unit of data classes in the ARM. Also the basic data types identified in the AIM. The term applies to single units which may be individual elements of geometry, individual elements of annotation, or collections of geometry or annotation elements that are combined to 


\section{Definitions}

form more complex data structures.

IGES POSTPROCESSOR - A software unit that transfers CAD information from the IGES format to the CAD database format of a particular system. The software is usually developed and maintained by a commercial CAD system vendor.

IGES PREPROCESSOR - A software unit that translates CAD information from the CAD database format of a particular CAD system to the IGES format. The software unit is usually developed and maintained by a commercial $C A D$ system vendor.

INFORMATION CONFIGURATION CONTROL - An approach that consists of specifying, documenting, and controlling both the creation and modification of information and the subsequent translation and exchange of the information between different systems and formats.

The approach requires substantial documentation for both the syntax (the format) and the semantics (the meaning) attached to an item of information.

INSTANCE - In general (e.g., from Websters): A detail or circumstance. In this AP an instance indicates that a specific member of an object class is being referenced. An example of an object class may be the assembly as identified by a drawing number. An example of an instance may be a completed assembly identified by a serial number.

LAYERED ELECTRICAL PRODUCT (LEP) - A broad category of electronic product types which are characterized by a two or more laminar strata stacked in sequential order. The completed structure serves to conduct electrical signals and may contain passive or active electrical functional elements. The structure is usually designed using two-dimension (2-D) graphics which result in photo-process tooling. Additional components or other LEPs may be added to complete the assembly. Descriptions within this document which apply additionally to products such as integrated circuits, printed circuit boards, flex harnesses are labeled with this term.

OCCURRENCE - In general (e.g., from Websters): Anything that happens or takes place. In this AP the term is sometimes used to label an object class which exists as a result of a sepcific grouping of two or more other object classes.

PRODUCT - A result produced by specified activities or used for specified activities.

PRODUCT DATA - The set of data elements that is necessary to fully support a product over its expected life cycle. The set of data elements includes all of the product definition data plus other data pertaining to the operation and maintenance of the product until it is removed from service.

PRODUCT DEFINITION DATA - The set of data elements that completely define a product for a certain discipline; a subset of product data. This set of data elements includes the geometry, topology, features, tolerances, and relationships necessary to completely define a component product or an assembly of products and is structured such that it can be used by one or more applications.

RELATIONSHIP - The identified association of two entities to each other. The two entities and their relationship taken together constitute a natural language role expressed in the data model. Example: Assuming the entities "Car" and "Person" are associated with relationship "Owned" in a data model Then the role may be expressed in natural language as: "A Car is owned by a person." The exact role stated depends on the modeling language's interpretation specifications. SEMANTICS - The meaning that is given or assigned to an item of information. The meaning is 
assigned to an item of information on the basis of its application area.

SYNTAX - The structure of expressions in a language. [3] This structure is described in a specification such as IGES.

UNIT of FUNCTIONALITY - A collection of entities, attributes and relationships that conveys one or more well-defined concepts (within the scope of the AP) such that removal of any component would render the concept(s) incomplete or ambiguous. (See "Current Issues on STEP APs,", 10/9/91.)

\subsection{LEP Application Definitions}

The bracketed character following the term indicates that the definition applies primarily to the AAM [A], AIM [I], or the ARM [R].

Note: definitions preceded by (T50) are from IPC-T-50E, December 1991.

ANNOTATION - [R] Text or legend pertinent to a design; text may appear as data related to legend on a multilayer substrate, lettering on a drawing, or other types of symbols.

APERTURE CODE - [R] Code representing aperture number used in plot file intended for a plotter such as a Gerber Plotter.

APERTURE NUMBER - The number for a photoplotter aperture setting, number used in pin and pad tables, and formatter table corresponding to a position on the aperture wheel.

APERTURE SHAPE - [R] Shape of aperture, such as one of the following: round, square, donut. APERTURE SIZE - [R] The $x, y$ dimensions of the aperture such as width or diameter where appropriate.

ART WORK (T50) - [R] An accurately-scaled configuration that is used to produce the artwork master used for production.

ART WORK GEOMETRY (target) (outside circuit) - [R] A collection of primitive shapes such as arcs, areas, and lines.

ART WORK TEXT - [R] Identifying text which calls out the product number and configuration number as well as program configuration for the art work. It identifies layer, revision, product number.

ASPECT RATIO (of Deposition Resistor) - [R] The ratio of the length of a thick or thin film resistor to its width, or the ratio between the resistance of the resistor and the sheet resistivity of the ink. This is also the number of effective "squares" in the design of a resistor.

ASSEMBLY CONSUMABLES - [R] A type of consumable that becomes part of the finished product. See also Consumable.

ASSEMBLY ID - [R] All information needed to uniquely identify a particular assembly, manufactured in-house or purchased. The identification may include, but is not limited to, the Drawing Number, Revision, Revision Status, Dash Number, Cage Number, case style, and other product notation.

ASSEMBLY OCCURRENCE - [R] Either a single product which is defined to become part of an assembly, or two products which are identified as items to be assembled into the next higher assembly. The occurrence identified may be noted in a bill of materials, or may be in-process 
work which must be identified for manufacturing tracking.

ASSEMBLY OCCURRENCE ID - [R] All information required to uniquely identify (ID) a product and the assembly it is assembled to. The identification may include, but is not limited to, the Drawing Number, Revision, Revision Status, Dash Number, Cage Number, case style, and other product notation.

ASSEMBLY TYPE - [R] The name of the class of assembly which indicated the general stage of completion of the electronic product. Examples are screened substrate, subassembly, component assembly, and final assembly.

ATTACHMENT - [R] Method used to describe how a component can be held on to the substrate such as solder, or epoxy.

ATTACH MATERIAL PATTERN - [R] Pattern associated with a component for the purpose of specifying the location and shape of an attachment medium or material.

AUTOINSERTION - An automated process by which through-hole components are selected, grasped, and positioned into the appropriate component holes in a PCB. Bonding of the component to the PCB may employ solder paste, glue or other consumables.

BACK PAD POLARIZATION - $[R]$ Description of which pin of a component such as a diode or IC chip is surface-mounted to an LEP.

BACK PAD POLARIZATION NAME - [R] Name associated with the back pad such as anode.

BILL OF MATERIALS - [R] A formatted list of products (instances of LEP Assembly Components) used for a particular assembly.

BLIND VIA - A via that is visible from only one side or the other of a design. (Derived from Via attributes.)

BONDING PADS - [R] Areas of metallization on the component and the LEP substrate that permits connection of the wire or circuit elements.

BURIED VIA - A via hole not extending to the surface. A buried via is completely contained within the inner layers of a design and therefore is not visible from the outside layers. May be referred to as an interstitial via hole. (Derived from Via attributes.)

CAD LAYER - [R] A method of separating information in a CAD system for the purpose of display or processing. Some CAD layers correspond to artwork layers.

CAGE NUMBER - [R] The military-assigned identification of the manufacturer responsible for the product definition.

CAPACITOR COMPONENT - [R] A kind of component used to add capacitance to a circuit.

CAPACITOR MATERIAL - $[R]$ The type-name for a capacitor derived from the dielectric material of the capacitor

CATCH PAD - $[R]$ Metallization patterns on a conductive layer associated with a via which aid in the interconnection of a via with its associated traces.

CHECKPLOT SCALE (T50) - [R] Drawing scale used on interim drawing for graphical data verification. (called a check plot.)

COMPONENT ATTACH - [A] The placing of a component (chip) on a substrate or header in a manner which causes it to mechanically bond. 
COMPONENT LOCATION PLACEMENT TOLERANCE - [R] The amount of displacement form the specified location which is acceptable.

COMPONENT LOCATION X Y - [R] The coordinates specified for the location of a component within an assembly.

COMPONENT ORIENTATION (T50) - [R] The direction in which the components on a printed board or other assembly are lined up electrically with respect to the polarity of polarized components, with respect to one another, and with respect to the board outline.

COMPONENT PLACEMENT RESTRICTION - [R] An area on substrate where components can not be placed.

COMPONENT POSITION - [R] The location at which a component is placed.

COMPONENT SELECTION - [R] A set one or more alternate chips to be specified in the design of an assembly, one of which is selected at the time of manufacture of a particular product.

COMPONENT TOLERANCE - [R] The range of acceptable component values; usually specified as a percentage of the nominal specified value.

COMPONENT VALUE - [R] The parameter associated with some component types which may be used in the fabrication of the components.

COMPONENT VALUE TOLERANCE - $[R]$ A range of permissible product's values.

COMPONENT VOLTAGE RATING - [R] Maximum voltage specified that can be placed across a component.

CONDUCTIVE PATTERN - [R] The configuration or design of the conductive material on the substrate. For a lep, the pattern includes conductors, lands, and through connections (vias) when these connections are an integral part of the manufacturing process.

CONDUCTIVITY, ELECTRICAL - [R] The capability of a material to carry an electrical current. The reciprocal of resistivity.

CONSUMABLE - $[R]$ An in-process or an end item material supplied in bulk form.

CONSUMABLE ID - [R] All information needed to uniquely identify a particular consumable, manufactured in-house or purchased. The identification may include, but is not limited to, the Drawing Number, Revision, Revision Status, Dash Number, Cage Number, and other material notation.

CONSUMABLE NAME - [R] The name given to bulk-purchase materials used in the assembly process, such as solder, flux, etc.

CONSUMABLE PROPERTY - [R] Properties of a consumable such as shelf life, expiration date. CONSUMABLE PROPERTY NAME - [R] The name of a Consumable Property.

CONSUMABLE PROPERTY VALUE - [R] The measurement value associated with a Consumable Property.

CONSUMABLE TYPE - $[R]$ The generic classification given to consumables, such as epoxy.

CROSSOVER - The transverse crossing of metallization paths without mutual electrical contact achieved by the deposition of an insulating layer between the conducting paths at the area of crossing. Dielectrics can be used with crossover conductive patterns, acting as a bridge over 
previously screened conductive material. (derived from trace geometry.)

DEFAULT DESIGN RULE - [R] The specifications which are followed during layout of the patterns on the layers of an electronic assembly. Examples are the maximum trace width, or the minimum conductor spacing.

DEFAULT DESIGN RULE DESCRIPTION - [R] The description of a Design Rule that is employed when no other rule is specified.

DEFAULT DESIGN RULE NAME - [R] The name associated with a Design Rule.

DEPOSITION COMPONENT - [R] Components which are fabricated coincidentally with the interconnects as opposed to components which are attached to the substrate after the interconnect network is complete.

DEPOSITION RESISTOR - [R] The deposition of resistor material which is formed as part of the conductive circuitry on a substrate layer. The resistor is a component in the sense of the electrical function of the circuit, but not in the sense of a separate product to be assembled to the completed substrate.

DEPOSITION SEQUENCE NUMBER - [R] A particular layer's position on the lep substrate with respect to the first layer.

DEPOSITION TYPE - [R] A category used to describe the technology used (THICK or THIN film) to create the component.

DERATING - [R] A factor which is applied to a product parameter to insure that reliability requirements are met; such as derate resistor power rating by $50 \%$.

DESIGN RULE - [R] A guideline that determines behavior with respect to specified design parameters. Becomes a constraint on the functional and physical layout. An example is a rule that ensures that minimum distance between elements in a design file is maintained.

DESIGN RULE CHECK (DRC) - [R] A process to enforce the design rules.

DESIGN RULE VALUE - [R] The measurement assigned to a particular restriction for a product's design.

DEVICE PIN ASSOCIATIVITY- [R] Functional relationships among pins that are needed to allow operations such as gate swapping or pin swapping to improve trace routing

DIE - [A] The uncased and normally leadless form of an electronic component that is either active or passive, discrete active device or integrated circuit.

DIE BONDING - [A] The attachment of an integrated circuit chip to a substrate or header.

DIELECTRIC - $[R]$ An insulating material used to separate conductors.

DIELECTRIC CONSTANT - The ratio of the capacitance, $\mathrm{Cx}$, of a given configuration of electrodes with a specified dielectric, to the capacitance $\mathrm{Cv}$, of the same electrode configuration having a vacuum as a dielectric.

DIELECTRIC LOSS ANGLE - [R] The difference between 90 degrees and the dielectric phase angle given a set of operating conditions. May be referred to as the dielectric phase difference.

DIELECTRIC OUTLINE - $[R]$ The boundary of an insulating layer.

DIELECTRIC STRENGTH - The maximum voltage that a dielectric can withstand, under 
specified conditions, without resulting in a voltage breakdown. Usually expressed as volts per unit length.

DISSIPATION - [R] The tendency for fluids or energy to distribute evenly within a medium. This tendency is usually associated with the ability of a material shape to remove heat or power from physical devices and circuits.

DISSIPATION FACTOR - [R] A measure of insulating or dielectric materials to absorb some of the energy in an alternating current signal.

DOCUMENTATION TEXT - [R] Words (strings) which exists on documentation.

DRAWING NUMBER -[R] The unique identification of a drawing within a manufacturing operation.

EDGE CONNECTOR - (from IPC: 'Edge-Board Connector' -) A patterned group of conductive terminals that is used specifically for making interconnections betweeen the LEP traces and the next higher assembly or among regions of the LEP. An edge connector may be a set of PCB features or a discrete component bonded to the PCB. Not all discrete connectors are mounted at the edge of the PCB (i.e. within a few trace widths of the PCB outline).

EE COMPONENT - (from AP 210 3.4.2) An occurrence of a part having electrical characteristics used in an assembled layered electrical product. Each EE Component has one or more circuit connections and a unique reference designator.

EE CONNECTIVITY - (from AP 210 3.4.6) The flow or association of EE_Signal information, or energy between objects in an electrical product. Connectivity focuses more on the logical need for a 'link' between two or among many terminals. The physical 'join' guides the packaging and testing of the physical conductors providing the required connectivity.

EQUIPMENT - [R] The identification name of machine types that are used as the means to complete a process step during manufacture of a device. Examples are a pick-and-place machine or a solder-joint inspection machine.

\section{ELECTRONIC PACKAGE - [R] (See Package.)}

FIDUCIAL [I] - See Registration Mark

FILE ID - $[R]$ The unique identification of a data file stored in or intended for a computer system. For files containing product information, the identification may include, but is not limited to, the Drawing Number, Revision, Revision Status, Dash Number, Cage Number, and other product notation.

FILM - (See Thick Film or Thin Film.)

FLM MATERIAL - $[R]$ Material used in thin and thick film processes to fabricate components and circuitry.

FLEX CABLE - A type of LEP which conducts one or more signals between attached devices (e.g., connectors) which is fabricated on non-ridged substrate material.

FLEX CIRCUIT - A type of LEP which is fabricated on non-ridged material.

FOOTPRINT - (from IPC: 'Land Pattern' -) A pattern of lands and associated features that defines the allowable interconnection space for the mounting, interconnection, and possibly the testing of a particular component. The lands in the footprint provide part of the connectivity between the 


\section{Definitions}

substrate conductors and the component terminals (or cell ports).

FUNCTION TYPE - [R] The category describing the use of a particular artwork geometry such as Pad, Registration Mark, Conductor, Dielectric Edge, Text.

GENERIC NAME - [R] A commonly accepted product identification, such as 54LS04 or 2N2222. GEOMETRY - [R] The constructs which are used to describe shapes of objects to be presented, or to which a product is to be fabricated. The description is usually in terms of a set of spatial coordinates and the identification of the curve-type to be constructed from the coordinates.

GEOMETRY ELEMENT - [R] A feature used to identify the meaning of, or use for, a collection of geometries. Examples from assemblies are countersink, keyway or threads. Examples from electrical planar designs are line, circle, area, etc.

GEOMETRY LINE WIDTH - [R] A measurement of the distance across a line (i.e., normal to the line). This value is often specified for circuitry artwork lines to regulate or check the actual or the artwork image produced from a computer plot or other image master.

GEOMETRY TYPE - [R] A classification of the basic geometry such as Line, Arc, and Polygon.

GLAZE - [R] An insulating film material used to protect underlying circuitry.

I HEAT SINK - (IPC: 'Heatsink' -) A mechanical device that is made of a high thermal-conductivity and low specific-heat material that dissipates heat generated by a component or assembly.

HYBRID MICROCIRCUIT ASSEMBLY (HMA) - In general an integrated circuit that is not monolithic. The term hybrid denotes that the circuit elements are made by two or more different technologies. A typical hybrid may consist of semiconductor chips and capacitors attached to a ceramic substrate with printed resistors and interconnections which are screen printed and fired.

INTEGRATED CIRCUIT (IC) - A type of LEP. An electronic part which has been fabricated upon a monolythic semiconductor substrate.

INTELLIGENCE - A capability to support queries about relationships among and values of uniquely specified layered electrical product data.

INTERNAL PART NUMBER - A part identifier unique within the assigning enterprise to enable distinctions based on the business needs of the enterprise.

JUMPER - A conductor expressly connected to a particular pair of circuit access locations to provide required connectivity.

KEEPIN - A design rule determining the definition of an area or volume within which specified features are to be contained.

KEEPOUT - A design rule determining the definition of an area or volume from which specified features are to be excluded.

LAND - [R] A portion of a conductive pattern (also called Pad by some) usually used for electrical connection, component attachment, or both, or a Via feature to one or more traces on the associated layer of the LEP.

LAYER - A strata of material such as conductor, dielectric, or resistor material which forms part of the LEP structure.

LAYER DISPLAY COLOR - [R] The display color of a layer on a color drawing or on a CAD machine. 
LAYER NAME - [R] The name of a particular layer (dielectric or conductive) such as Glaze or top conductor.

LAYER SEQUENCE NUMBER - [R] A particular layer of material (conductor or dielectric) position on the LEP substrate with respect to the first layer. Usually begins with the first material placed on a substrate. These layers relate to process layers as contrasted with e.g., CAD layer.

LAYER TYPE - [R] A category used to describe the function performed by a deposited layer such as Conductor, Dielectric, Fill, Glaze, Resistor, Epoxy, Solder.

LAYER RESTRICTION - [R] A design restriction which is imposed on a particular layer of the product.

LEAD FRAME - [R] The metallic portion of the device package that completes the electrical connection path from the die or substrate conductors to the external circuitry.

LEAKAGE CURRENT - [R] The flow of electrons through the dielectric of a capacitor, usually expressed as a maximum which will be acceptable for the component.

LEP - [R] See Layered Electrical Product (Section 3.1).

LEP ASSEMBLY COMPONENT - [R] A product or an assembly which has been identified and located within an assembly.

LEP CAD PRESENTATION - [R] The design file that contains the electronically coded data available on a particular design.

LEP ID - [R] All information needed to uniquely identify a particular product, manufactured inhouse or purchased. The identification may include, but is not limited to, the Drawing Number, Revision, Revision Status, Dash Number, Cage Number, case style, and other product notation.

LEP NAME - [R] A descriptive name given to a product that usually describes its function.

LEP NUMBER - [R] The product number of a particular type.

LEP SUBSTITUTE PART NUMBER - [R] Other organizations identification (i.e., the customer may have alternate product number for the same product).

LEP VERSION - $[R]$ The revision level of a product; each change to the product design results in a new product Version.

LEVEL OF PREFERENCE - [R] A relative rating assigned to a product in a collection of functionally similar products which indicates its ranking of desirability for use in a product to be produced for the rating organization.

LIBRARY NAME - [R] The name of part in CAD system files of part information.

LINE RESISTANCE - The resistance of a conductor line on a substrate measured in total trace resistance.

LINE WIDTH - [R] (See Geometry Line Width.)

LOG REQUIREMENTS - [R] Indicates what information about the process step must be recorded and retained such as Machine $\mathrm{D}$, Component Lot Identification, Start Time, Stop Time.

LOSS FACTOR - [R] A property of an insulating material that is equal to the product of its dissipation and dielectric constant.

MANUFACTURER NAME - [R] The name a company is registered to do business under; may 
also include a company division name.

MAX SUBSTRATE SIZE - [R] The largest size of substrate which can be accommodated.

$\mathrm{MIL}$ - [R] A unit equal to 0.0254 millimeter (mm), (or $0.001 \mathrm{in}$ ).

MOUNTING_HARDWARE - Fasteners applied to attach one or more Pca_component occurrence(s) to the PCB or the PCB to its next assembly.

MOUNTING PAD - $[R]$ Metallization pattern on the substrate associated with a component for the purpose of indicating the region for physical attachment of the component to the substrate. See Attach Material Pattern.

MULTICHIP MODULE -[A] A complex multilayer circuit containing components (integrated circuit chips) which cover at least half of the available circuit area. Note that this term is not present in the CAD information.

MULTI-UP ID - [R] The unique identification of data which indicates the placement of an array of images nested together on a single photo tool artwork. For files containing product information, the identification may include, but is not limited to, the Drawing Number, Revision, Revision Status, Dash Number, Cage Number, and other product notation.

NET - An entire sequence of electrical connections from the first source point to the last target point, including lands and vias. The Net is identified by a Net Name which is unique within the product, and may also have a signal name.

NET DESIGN RULE - [R] Specified constraints on parameters related to the connectivity. Examples are maximum distributed inductance, or maximum circuit length.

NET DESIGN RULE NAME - [R] The assigned identification of a specification for some parameter of all conductive elements associated with an individual signal.

NET DESIGN RULE VALUE - [R] The measurement allocated to some parameter of all conductive elements associated with an individual signal.

NETLIST - A to-from listing of all the interconnections in the design. The netlist should correspond to the to-from listing of all the wires in the schematic. (Model; set of Net.)

NET NAME - A character string used to identify and distinguish nets; the name of the logical connection realized by the trace.

OBSTRUCTION - A physical entity which restricts where a component or a trace can be placed. See also Component Placement Restriction.

ORIENTATION - [R] The angle of rotation of a component relative to a predetermined direction.

PACKAGE - An integrated circuit, or a LEP circuit. It provides hermetic or nonhermetic protection, determines the form factor, and serves as the first-level interconnection externally for the device by means of package terminals.

PACKAGE DIMENSION - The physical measurements of the package. See Part Outline.

PACKAGE ID - $[R]$ The unique identification of a component enclosure. For files containing product information, The identification may include, but is not limited to, the Drawing Number, Revision, Revision Status, Dash Number, Cage Number, case style, and other product notation (e.g., TO-18; a JEDIC Standard number for a metal transistor package).

PACKAGE OUTLINE - [R] The 3-dimensional curve and surface geometry of a component pack- 
age.

PACKAGE PIN LOCATION - [R] The geometric position of a lead in a part package of an electrical product with respect to the part origin and reference axis.

PACKAGE PIN NAME - [R] A referencing word that describes a use for the lead of a component package; such as "Output1".

PACKAGE PIN NUMBER - [R] The identifying number assigned to the lead of a package for electronic components.

PACKAGE PIN TYPE - [R] The name given to the lead of a component package which indicates how the lead is to be attached to the assembly.

PAD - The metallized area on a substrate or on the face of an integrated circuit used for making electrical connections. Also see land.

PAD END FEATURE - [R] The type of joint formed on a wirebond wire at the place where the wire attaches to the circuitry of an assembly.

PAD TYPE - [R] The descriptive name that implies the function of a pad.

PADSTACK $[I]$ - An ECAD construct for all of the pieces of information (features) about a component pin needed to establish the interconnection /layer geometry on the LEP.

PAD WIREBOND END FEATURE - [R] The method of attaching a wirebond to a pad as denoted by the appearance of the joint such as wedge, ball.

PANEL MULTI-UP (PANEL) - [R] A fabrication artifact (often with test coupons) intended to contain one or more printed board details. A panel may be defined to realize process efficiencies or to minimize wasted material. I.e., multiple of the same image on one piece of material that will be separated after processing into multiple substrates

PART - [A, R, I] An individual functional element in a physically independent body that cannot be reduced or divided without destroying its stated function. When assembled onto the completed film network will form part of the circuit assembly. In a CAD system, represented as a graphic symbol with attached annotations. Parts are manufactured items; the information about which is usually stored in a library or listed in a catalogue.

PART GRAPHIC- [R] The graphic presentation of a component.

PART ID - [R] All information needed to uniquely identify a particular item, manufactured inhouse or purchased. The identification may include, but is not limited to, the Drawing Number, Revision, Revision Status, Dash Number, Cage Number, case style, and other product notation. PART KIND - [R] A category of component describing how it is used electrically such as mechanical, passive, active.

PART MANUFACTURERS NAME - [R] The corporate name used by the manufacturer of components.

PART NAME - [R] Description of the function of the component such as Coil, Cover, IC Chip, Transistor, Resistor Header, Substrate.

PART OUTLINE - [R] The boundary of component on the top assembly drawing or the CAD equivalent.

PART PROPERTY - [R] A pair of terms, one indicating the property name (see Component 


\section{Definitions}

Property Name), the other the value of the property (see Component Property Value).

PART PROPERTY NAME - [R] The name of a measured parameter associated with a device used in an assembly. Examples are capacitance, inductance, gain, resistance, and temperature range.

PART PROPERTY VALUE - [R] The value associated with a Component Property. Examples would be $10 \mathrm{~K}$ as a value for the resistance property, or 50 as a value for the low frequency gain property of a semiconductor.

PASSIVE COMPONENT - [R] A component which does not exhibit signal gain but does affect one or more other parameters of a signal. Examples are resistor, inductor, and capacitor.

PATH VERTEX - The X, Y location which specifies one end point of the linear segment of a trace.

PATTERN [R]- The configuration of materials on a panel or printed board layer. Pattern also denotes the circuit configuration on related tools, drawings, and artwork masters.

PATTERN ID - $[R]$ The unique identification for the artwork for a specified layer of a laminated product.

PATTERN VALUE - [R] The measurement value assigned to a deposited or etched component at some specified stage in the fabrication cycle. An example is $2300 \mathrm{ohms}$ prior to trimming.

PATTERN VALUE MIN \& MAX - [R] The tolerance associated to the deposited or etched component.

PHOTOLITHOGRAPHY [A]- The use of light or ultraviolet rays through a mask to define circuit conductor patterns. (Model; intractable taxonomy.)

PHOTOPLOT APERTURE- Mechanism which allow a certain amount and incidence of light to expose film, thereby making a "photograph" of design being plotted. See Aperture Size.

PHOTORESIST [A]- A photosensitive coating that is applied to a laminate and subsequently exposed through a photo tool (film) and developed to create a pattern that can be either plated or etched.

PHYSICAL LAYER (1 top) - A conductor, dielectric, or fill for each layer.

PICK/PLACE - An automatic process by which SMT parts are selected, grasped, and positioned onto an LEP.

PIN - The identification of a possible connection to a component providing a reference point for creating a netlist.

PIN ASSIGNMENT - [R] The name of the characteristic or electronic function assigned to the lead of a component package in a circuit.

PIN END FEATURE - [R] The type of joint formed on a wirebond wire at the place where the wire attaches to a component.

PIN LOCATION - The physical location of pins on a component package with reference to its assembly origin.

PIN NAME - $[R]$ The general functional notation for the signal role at the lead of a component 
package such as $\mathrm{E}, \mathrm{B}, \mathrm{C}$ on a transistor.

PIN NUMBER - A number used to distinguish pins on a component or package.

PIN TYPE - The name of a characteristic on a pin, such as input, output, tri-state analog.

PIN WIREBOND END FEATURE - [R] The characteristic of the wire at a joint with the material to which the wire is attached. The usual characteristics given are wedge or ball.

PLACEMENT NAME/PHYSICAL NAME - The name of component in CAD library.

PLACEMENT TOLERANCE - [R] The permitted positional variation of a component on an assembly.

PLANE - A portion of a roughly planar physical conductive layer of an LEP assigned a particular function or signal implementation, i.e. Ground_Plane, Signal_Plane, and Power Plane.

PLATING - (1) The metallic deposit on a surface, formed either chemically or electrochemically.

(2) the process of the chemical or electrochemical deposition of metal on a surface. (Model;

Deposition taxonomy.)

POINT - $[R]$ A singular location in a given coordinate system.

POINT X VALUE - [R] The numeric value in one (usually horizontal when viewed from a reference coordinate system) direction from a single point spatial reference.

POINT Y VALUE - [R] The numeric value of a single point reference, in a direction orthogonal to the $\mathrm{X}$ vector direction.

POWER DENSITY - [R] A measure of the concentration of energy, such as 1 watt per square inch.

POWER DISSIPATION - (T50) [R] In watts/meter ${ }^{2}$ (heat flux density), the energy used by an electronic device in the performance of its function.

POWER PLANE - A conductive area or set of closed areas on a particular physical layer of an LEP assigned the function of distributing supply voltage to the appropriate ports of components of the LEP.

PREFORM - [R] For soldering or adhesion functions, a form which is punched out of thin sheets of solder, epoxy, or eutectic alloy. The form is placed on the spot of attachment by soldering or by bonding prior to placing the object there to be heated. (a type of Part Name)

PRIMITIVE TYPE - [R] The category of simple geometric element that makes up artwork such as geometry or text.

PRINTED CIRCUIT ASSEMBLY - A PCB together with attached components.

PRINTED CIRCUIT BOARD - The Printed Circuit Board (PCB) is a processed substrate which provides most of the electrical connections among printed and packaged components. The PCB may be single-sided, double- sided, or multi-layered and may be rigid, flexible, or rigid-flex.

PRINTED WIRING ASSEMBLY - see Printed Circuit Assembly.

PRINTED WIRING BOARD - see Printed Circuit Board

PROBE PAD - A connection point for an external probe used to test some aspect of the LEP circuit function. For example, a probe pad may be used to make connections to a screened resistor during the trimming process. 
PROCEDURE - [R] A step-by-step description of the activities needed to complete a given operation.

PROCESS ID - $[R]$ The unique identification of the parameters used on one process step during manufacturing. The identification may include, but is not limited to, the Drawing Number, Revision, Revision Status, Dash Number, Cage Number, and other product notation.

PROCESSING CONSUMABLE - [R] Material or chemicals which are used in manufacturing processes which do not remain as part of the product being manufactured. Examples include cleaning fluids, flux, and photoresists.

PROCESS DESCRIPTION - [R] The narrative explanation of an identified step in the fabrication of a product or assembly.

PROCESS OPERATION - [R] The procedures sequentially described by a document identified by the Process $\mathrm{ID}$.

PROCESS STEP - [R] A single operation used to manufacture an Assembly Occurrence. Each step corresponds to one block on a flow chart.

PROCESS STEP NUMBER - [R] A sequential number assigned to a process step.

PROCESS VARIABLE NAME - [R] The identifier assigned to a particular fabrication step.

PROCESSING CONSUMABLE NAME - [R] The generic identification of a substance used during the fabrication of a product or assembly, but which does not remain as part of the product. Examples are flux, solvent, or resist.

PRODUCT CONSUMABLE - [R] Material or chemicals which are used in manufacturing processes and which remain as part of the product being manufactured. Examples include solder, epoxy, and plated metals.

PRODUCT CONSUMABLE NAME - [R] The generic name of material or chemicals which is used in manufacturing processes and which remain as part of the product being manufactured.

PRODUCT TOLERANCE - [R] The allowable deviation from the specified location of a product feature.

PRODUCTION TOLERANCE - [R] The allowable deviation from the specified location of a product feature.

PROGRAM - [R] A text string indicating the system a LEP was designed for or intended to be used in.

QUALIFIED VENDOR - [R] A company from which components are purchased and which has been identified as meeting specified requirements for certification and/or inspection of the components being sold.

REFERENCE DESIGNATOR - [R] A character string which defines an instance of an assembly component; unique within the product or system. The guidelines generally followed are found in ANSI Y32.16-1975 (e.g., R1=Resistor 1, U7=Microcircuit 7, A10= Assembly 10; thus A10R6 is the 6th Resistor in Assembly 10).

REGISTRATION - The proper alignment of layers with respect to other layers or to the substrate. The accuracy of registration is measured as the concentricity or relative position to a datum.

REGISTRATION MARK - $[A, R]$ The marks on a wafer or substrate that are used for aligning 
successive processing masks. Also known as alignment marks or fiducial marks.

REGISTRATION MARK NAME - [R] The term assigned to a locating orientation feature related to a particular use.

RESISTIVITY - The ability of a material to resist the passage of electric current. See also Resistor, Sheet Resistance or Line Resistance.

RESISTOR - A device that offers resistance to the flow of electric current in accordance with Ohm's law: $R=E / I$, where $R$ is resistance, $E$ is voltage, and $I$ is current.

RESISTOR GEOMETRY - The film resistor outline. The four system generated geometries for many CAD systems are as follows: rectangle, tophat, $\mathrm{u}$ - shaped, serpentine.

RESISTOR MAX ASPECT RATIO - A greatest-value factor obtained by dividing the specified width by the length and multiplying the numerator and denominator by a constant such that the resulting factor is a simple number, such as $4: 1$. Such a design rule may constrain the automated design system, and is transferred with the design. The rule is not necessarily intended to be applied to the finished product.

RESISTOR MIN ASPECT RATIO - A least-value factor obtained by dividing the specified width by the length and multiplying the numerator and denominator by a constant such that the resulting factor is a simple number, such as 1:6. Such a design rule may constrain the automated design system, and is transferred with the design. The rule is not necessarily intended to be applied to the finished product.

RESISTOR MIN LENGTH - A design rule-or metric - which establishes the least acceptable dimension a resistance trace length, such as 40 mil. Such a design rule may constrain the automated design system, and is transferred with the design. The rule is not necessarily intended to be applied to the finished product.

RESISTOR MIN WIDTH - A design rule-or metric-which establishes the least acceptable dimension across a resistance trace, such as $20 \mathrm{mil}$. Such a design rule may constrain the automated design system, and is transferred with the design. The rule is not necessarily intended to be applied to the finished product.

RESISTOR OVERLAP - A thick-film conductor pad that overlaps and makes contact with a thick-film resistor area.

RESISTOR PASTE VALUE - [R] Material used to create thick film resistors rated with a nominal ohm per square value.

RESISTOR SHAPE - $[R]$ The term which is characteristic of the topology used for a deposited or etched resistor component.

RESISTOR TERMINATION - The contact area between a thick- or thin-film resistor and an adjacent conductor layer.

REVISION - [R] Number(s) and/or character(s) that uniquely identify a version of documentation and the products built to that documentation.

ROUTE REGION - (T50) Region where routing paths can automatically be placed between points to be interconnected.

SCHEMATIC - (T50) A drawing that shows, by means of graphic symbols, the electrical connections, components, and functions of a specific circuit arrangement. 
SCHEMATIC ID - [R] All information needed to uniquely identify a particular circuit functional diagram, manufactured in-house or purchased. The identification may include, but is not limited to, the Drawing Number, Revision, Revision Status, Dash Number, Cage Number, case style, and other product notation.

SCREEN ID - [R] All information needed to uniquely identify a particular screen, manufactured in-house or purchased. The identification may include, but is not limited to, the Drawing Number, Revision, Revision Status, Dash Number, Cage Number, and other product notation.

SCREEN PRINTING - [A] A process for depositing material in a pre-defined pattern by forcing material through a stencil screen with a squeegee.

SCREEN STENCIL - [A] A network of metal or fabric strands, mounted snugly on a frame, and upon which the film circuit pattern and configurations are superimposed by photographic means. (from ISHM Industry Guide, 1990)

SHEET RESISTIVITY - [R] Electrical resistance of a thin sheet of a material with a uniform thickness as measured across opposite sides of a unit square pattern expressed in ohms per square. (from ISHM Industry Guide, 1990)

SIGNAL CONDUCTIVE PATTERN - [R] The association of geometry and the electrical characteristic that is intended to be carried on the material finished to that geometric shape.

SIGNAL NAME - [R] The user defined functional name, usually referring to an electrical impulse of predetermined voltage, current, polarity, and pulse width. See also Net Name.

SILK SCREEN - A physical layer of the LEP providing text and/or graphic annotation by means of screen printing. The resulting annotation is usually visible from the top side or from the bottom side of the LEP. (IPC: 'Screen Priniting') - The transferring of an image to a surface by forcing a suitable medium with a squeegee through an imaged-screen mesh.

SMT - Surface Mount Technology (IPC: 'Surface Mounting' -) The electrical connection of components to the surface of a conductive pattern that does not utilize component holes.

SURFACE MOUNT - see SMT.

SOLDERING [A]- A process of joining metallic surfaces by melting solder.

SPECIFIED TOLERANCE - [R] The amount of (usually) dimensional deviation which has been called for in the product specification.

START LAYER - [R] The first of one or more layers through which a via or passage will pass.

STATION - [R] The place or the equipment which is identified (in planning) as the location of a processing or an assembly step during the manufacturing of a product.

STEP X COUNT - [R] The number of patterns in X direction in panel multi-up.

STEP X DISTANCE - [R] The $X$ step distance between patterns in panel multi-up.

STEP Y COUNT - [R] Number of patterns in Y direction in panel multi-up.

STEP Y DISTANCE - [R] The Y step distance between patterns in panel multi-up.

STOP LAYER - [R] The last layer through which a via or passage will pass.

SUBSTRATE [R]- The supporting structural material into and/or upon which the passivation, metallization and circuit elements are placed. MIL-STD-883C (Usually aluminum oxide (alumina)). 
SUBSTRATE OUTLINE [R]- The graphical representation of the substrate shape.

SUBSTRATE MATERIAL - [R] The name of the substance from which the base layer is formed. SUBSTRATE THICKNESS - [R] The nominal material cross section of the base material of a laminated product.

SURFACE RESISTIVITY - [R] (See Sheet Resistivity.)

SWAPPING - [A] The process that exchanges connections from one pin to another pin to improve routing, provided the pins are in the same gate and in the same swap group.

TEMPERATURE COEFFICIENT OF CAPACITANCE - [R] A multiplier which represents the linear change of a capacitance value per degree change in temperature. It may be positive, negative, or zero and is usually expressed in parts per million per degree Celsius, or $10^{-6}$.

TEMPERATURE COEFFICIENT OF RESISTANCE - [R] A multiplier which represents the linear change of a resistance value per degree change in temperature. It may be positive, negative, or zero and is usually expressed in parts per million per degree Celsius, or $10^{-6}$.

TERMINATOR MOUNTING - [A] Metallization pattern on the substrate associated with a film component. The purpose of the pattern is to indicate the region for electrical connection of the film component to its connecting traces.

TEST - $[R][A]$ The examination of a product during or after fabrication for conformance to specified criteria for that product.

TEST CONDITION - [R] The specification of the setup needed to carry out a specific test of a product. Examples include supply voltage, temperature, and initializing signals.

TEST COUPON (IPC: -) A portion of quality conformance test circuitry that is used for a specific test, or group of related tests, in order to determine the acceptability of a product.\} (Test Coupons are often provided to enable detailed testing of adherence, positioning, plating, etching, and other process attributes even if some of the tests are destructive.)

TEST DESCRIPTION - [R] The narrative which is followed in performing the specified test.

TEST MAXIMUM - $[R]$ Value of the upper limit of a range of test values.

TEST MINIMUM - [R] Value of the lower limit of a range of test values.

TEST POINT (from IPC: -) A designated point of access to an electrical circuit that is used for electrical testing purposes.

TEST SEQUENCE NUMBER - [R] A number assigned to a test in a series of tests.

TEST SYMBOL - $[R]$ Short character sequence that allows easy reference to test such as Vin.

TEST TOLERANCE - $[R]$ The acceptable deviation from the specified test results parameter.

TEST TYPE - [R] Category of test being performed such as Final, Pre-burn, Qual, Sample.

TEST UNITS - $[R]$ Measurement units of the Test Minimum and Test Maximum values for the test.

TEXT - [R] An ordered sequence of characters (glyphs) to which a meaning is ascribed.

TEXT ATTRIBUTE - [R] The characteristics that describe the appearance of text such as size, font, slant, layer. 
THERMOCOMPRESSION BONDING - [R] The process involving the use of temperature and pressure to join two materials by interdiffusion across a boundary. (from ISHM Industry Guide, 1990)

THERMOSONIC BONDING - $[R]$ The process involving the use of temperature, pressure and applied ultrasonic energy to join two materials by interdiffusion across a boundary. (from ISHM Industry Guide, 1990)

THICK-FILM CIRCUIT - [R].A circuit that is fabricated by the deposition of paste materials such as screen-printed ceramic material (cermet) pastes on a ceramic substrate, which are fired in a kiln to create permanent circuit patterns.

THIN-FLM CIRCUIT - [R] Conductive, resistive, or dielectric material, usually less than 50,000 angstroms in thickness, that is deposited onto a substrate by vacuum evaporation, sputtering, or other means. (from MIL-STD-883C, Notice 12)

THRU-Hole - see HOLE. ("Thru" is a spelling variant of the word "through.")

TOOL - [R] (See Tool Type.)

TOOL DESCRIPTION - [R] A narrative which indicates the use of the implement.

TOOL ID - [R] All information needed to identify uniquely a particular manufacturing implement-manufactured in-house or purchased. The identification may include, but is not limited to, the Drawing Number, Revision, Revision Status, Dash Number, Cage Number, and other product notation.

TOOL TYPE - [R] The name given to a device used, as part of product manufacturing, to accomplish an operation. Examples include the product traveler, a cutting device, or a machine.

TOOLING HOLE - (from IPC: -) A tooling feature in the form of a passage in an LEP or fabrication panel which may often be a non-plated hole for fixturing, measuring, or testing by means of the measuring feature(s) provided by the passage.

TRACE (IPC: 'Conductor' -) A single conductive path in a conductive pattern. 'Conductive Pattern' - The configuration or design of the conductive material on a base material. (This includes conductors, lands, vias, heatsinks, and passive components when these are an integral part of the printed board manufacturing process.) The role of 'join' which provides physical connections is the common characteristic here.

TRACE KEEPOUT - [R] Symbolic representation of a region (layer-specific) associated with component which may not be used for trace routing.

TRACE LAYER - [R] The physical layer on which the given trace exists.

TRACE WIDTH - [R] The physical width of the trace segment. The width data is typically used to control the photoplotter aperture or the pattern generator setting.

TRACE WIDTH TOLERANCE - [R] The allowable range of the dimension across a conductive line.

TRIM PATH - $[R]$ The path made by trimming (using an abrasive or a laser) a component to obtain the design value.

UNITS (e.g., Volt, Meter) - [R] A definite amount or quantity used as a standard of measurement. USER DEFINED PROPERTIES - [T] Structured data describing information that is not 
previously defined. This property is often used for information not yet defined for the data structure.

USER INFORMATION - [R] Unstructured text information inserted as user comments, such as drawing notes.

VACUUM DEPOSITION - The deposition of a metal film onto a substrate in a vacuum by metal evaporation techniques.

VARIABLE VALUE - [R] Quantitative data that is associated to a particular named data element. VENDOR ADDRESS - [R] The postal location of the organization from which items are procured.

VENDOR NAME - [R] The identifying text of the company, which may include the company division, that supplied materials, equipment, or service.

VIA - An electrically conductive passage connecting two or more lands on distinctly specified layers of an LEP.

VIA HOLE - [R] A hole (i.e., a void or passage) through insulating layer (s) used to make a connection (electrical or thermal), but for which there is no intention to insert a component lead or other reinforcing material. The hole may be any shape as defined by a Via Shape.

VIA KEEP OUT - [R] The symbolic representation of a region (layer-specific) associated with a via where traces cannot exist.

VIA LAYER - [R] A layer which is used principally (in a CAD system) for placing vias holes.

VIA LOCATION - [R] The X, Y position on which a via is positioned.

VIA SHAPE - [R] The shape of the top view of a via.

VIA SIZE - [R] The width and length or the diameter of a via.

VOLTAGE ALLOWANCE - [R] A design rule that requires extra spacing between specified traces (e.g., to allow higher voltages to be carried).

VOLUME RESISTIVITY - [R] The volume resistance between two electrodes (of unit area and unit distance apart) that are in contact with or embedded in a specimen. The value is the ratio of the direct voltage applied to the electrodes in proportion to the current between them that is distributed through the volume of the specimen. Usually expressed in ohms per centimeter.

WIRE BOND - [A, R] A completed wire connection that provides electrical continuity. Bonding wires are used to connect between component pads, or package pin, or conductive pads on the LEP substrate.

WIRE BOND MAX LENGTH - [R] A design rule-or metric-to limit total length of a wirebond wire, such as 140 mils maximum.

WIRE BOND MIN LENGTH - [R] A design rule-or metric-to limit the least-length parameter of a wirebond wire, such as 10 mils minimum.

WIRE BONDING - [A] The method used to attach very fine wire to make electrical connections. WIREBOND SEQUENCE NUMBER - [R] The number assigned to a wirebond that specifies the order in which wirebond is to be made.

WIRE SIZE - [R] The diameter of wire, such as $0.8,1,5$ mils. 
Definitions

\section{Blank Page}




\section{INFORMATION REQUIREMENTS AND APPLICATION REFERENCE MODEL}

In this section, LEP information needed for the documentation and manufacture of the end item is described in the Application Reference Model (ARM). In addition, the task of identifying collections (or groupings) of information, termed Application Information Requirements, has been assembled into categories and described in section 4.1. In section 4.2.1, the methodology used to convey information and the method's implied constraints, are described. Lastly, in section 4.2.3, the results of utilizing the method to capture all of the data entities (i.e., information primitives) as they relate to each other are diagrammed.

It should be noted that information that describes the logical information structures required for accomplishing the physical implementation of an associated Application Reference Model is called an Application Interpreted Model (AIM) and is found in section 5. The indication of when the information is used during the overall design and manufacturing process is documented in the Application Activity Model (AAM) in Appendix A.

\subsection{Application Data Views}

Units of Functionality was an organization of data with a focus on the activities that are to be performed. A new construct, Application Information Requirements (see Section 2.1) has been created to shift the emphasis from the activities to the information needed to support those activities. In this context we are using information to mean both the data and the meaning (semantics) of that data.

\subsection{Application Reference Model}

The Application Reference Model was created to define the semantics (data and relationships) of all information needed for the manufacture and documentation of the end item product. The model may relate to information which is not typically captured during design. Examples are the Test environment (TEXT STRING) supplied in a procurement drawing, or the actual hand-written signatures found in the drawing title block. The model does not include data related to enterprise management or to the use of the LEP.

\subsubsection{Descriptions of the ARM Data Constructs}

The following sub-sections define the interpretation methods which shall be followed. These subsections are not intended to provide a tutorial on the subject. For further explanation of the syntax and methods the reader is directed to manuals, information, and tools references available from the IDEF-Users Group, 1900 Founders Drive, Kettering, OH 45420; telephone (513) 259-4702

\subsubsection{ARM Methods and Language}

The ARM is presented in the $\mathrm{IDEF}_{1} \mathrm{X}[13]$ language. $\mathrm{DEF}$ derives its name from the activity that developed the methodology, the Air Force Integrated Computer Aided Manufacturing (ICAM) Definition Program. The program developed several languages. $\mathrm{DEF}_{1} \mathrm{X}$, was developed to model data-the semantics of information. A data model may be considered as a connecting link between the user's view of the data(referred to as the external schema) and the manner in which the data is stored in a computer, or database (the internal schema). Common to these two views of the 
information is a conceptual schema and the data model is the visual presentation of the conceptual schema. In general, the data model is presented as a series of drawings that contain diagrams and accompanying text. The text forms a glossary that defines the terms used in the diagrams and more fully explains their relationships and context.

The component parts of an IDEF model are the entities, their attributes and their relationships as illustrated in figure 4-1. An entity is an item (an object or an event) about which we wish to keep data. In general, rectangles are used to graphically represent entities. If the entity is not within the scope of the present model, the outline of the entity is dashed, notifying the reader to look elsewhere for the definition of the entity. Each entity box has a name (the entity-class label) that is given above the rectangle and which is defined in the model glossary.

Attributes of an entity are shown inside the entity rectangle. An attribute will have one value

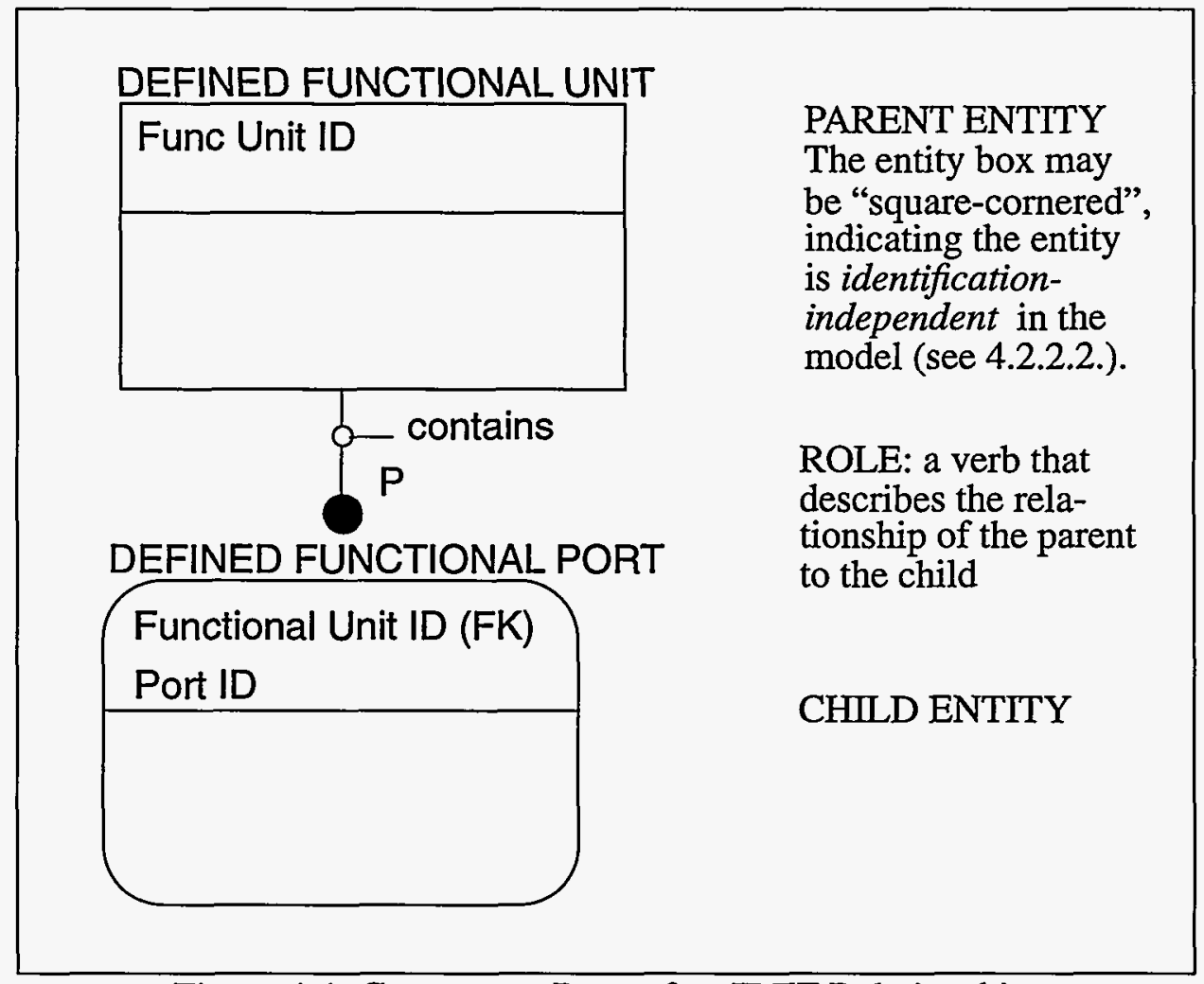

Figure 4-1. Component Parts of an IDEF Relationship.

(instance) for each instance of the subject entity. Some attributes that uniquely define the instance of an entity, called keys, are located above a line near the top of the entity rectangle. Keys are of several types and the general concepts of key migration follow those concepts as used to develop relational database structures. For example: the key attribute set from the parent entity is propagated downward through the model structure and such instances are called foreign keys. Attributes that are not keys are located below the horizontal line of the entity rectangle.

The relationships between entities are shown by the lines drawn between them in the model diagram. The relationship consists of the role and the cardinality. Text associated with the interconnecting lines provides a clear meaning (the role or predicate) of the relationship between the entities. One end of the line is connected to the entity rectangle denoting that this entity is the 
independent entity. At the other end of the line-at the dependent entity end-a terminator symbol (a "big dot") may carry cardinality information on how to read the model as in figure 4.2. If there is no letter adjacent to the terminator symbol, this means that there may be "zero, one or many" entities associated with the relationship. Likewise, the letter $\mathrm{Z}$ designates "exactly zero or one," P designates "one or many," and the letter n designates some exact integer number of occurrences.

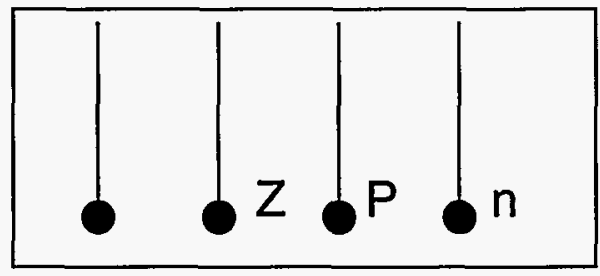

Figure 4-2. Cardinality.

Lastly, models may contain information that denote the "categorization" or types-of entities as diagramed in figure 4-3. Each instance of the generalization parent must be one of the types. A double horizontal bar indicates that all types of dependent entities are present (for example, all people are either male or female). If all of the various types of entities are not listed (the model equivalent to a written ellipsis (...)), then a single horizontal bar is used to show that there may be relationships not shown and the categorization is assumed to be incomplete.

A related pair of entities are read, that is, retrieved as two English sentences, in the form of assertions. The pair may be read in both directions. From the top down (i.e., toward the entity at the big dot end of the relation line), the set is read as follows: the independent entity becomes the subject; the relation label becomes the predicate; and the dependent entity becomes the object in the assertive sentence. From the bottom up the sentence is constructed using the dependent entity as the subject and the independent entity as the object; the usual relation is "always has exactly one."

Additional details may be obtained that will enable those who are interested in working with IDEF to become more proficient at reading the models. Several good references[15, 16] are available.

\subsubsection{Data Construct Definitions}

The definitions of entities for the Layered Electrical Product ARM are found in section 3.2.

\subsubsection{Data Construct Assertions}

The method and language $\left(\mathrm{IDEF}_{1} \mathrm{X}\right)$ used for this AP ARM carries several assertions important to the interpretation and application of the ARM.

Attributes:

All attributes which appear within the entity box exist whenever an instance of the entity occurs, unless designated by the (null $\mathrm{OK}$ ) notation. 


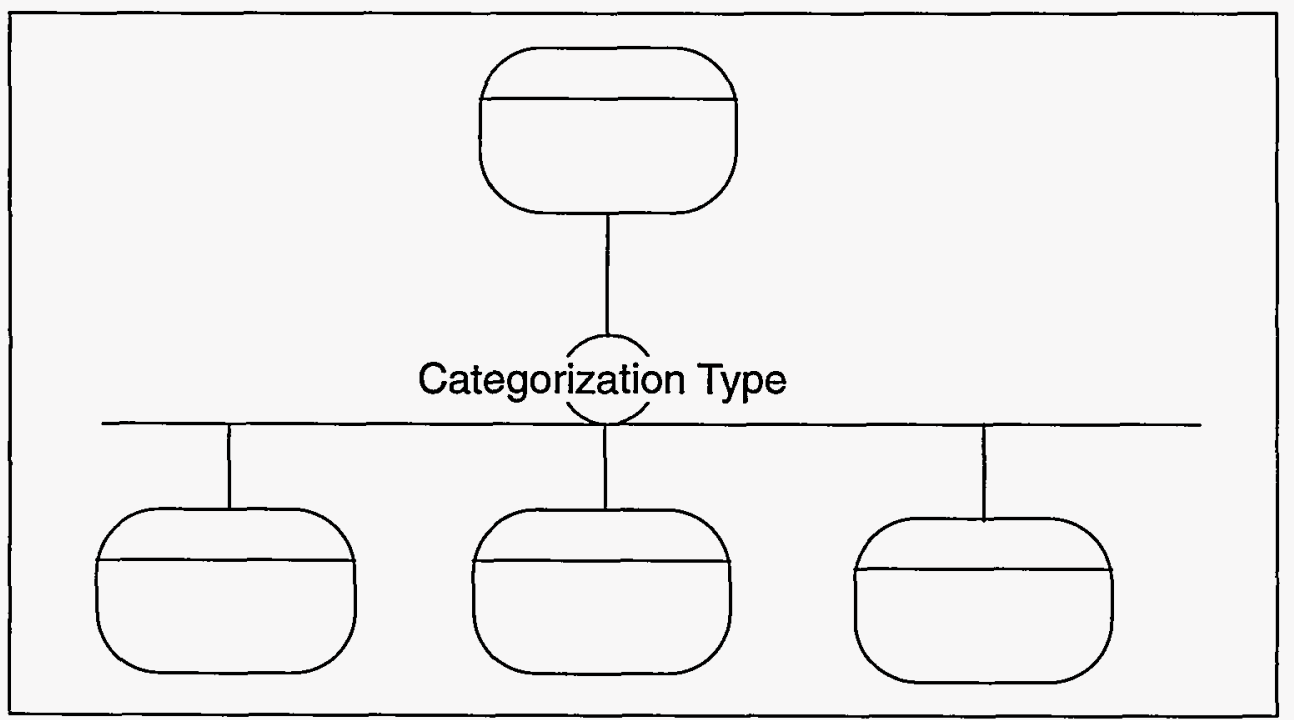

Figure 4-3. The Categorization Structure.

Relationships:

The ARM is intended to be interpreted independently of any computer database structure. Thus, the entities may be considered as representing objects in an Object Oriented (OO) system, or as tuples in a Relational system. As such, the keys have not been migrated as required by relational notation. The relationships (connecting lines) may be used to define $O O$ methods, or to migrate relational foreign keys. The entity at the attached end of a relationship line is considered the parent of the two entities. The following dependencies apply to the parent-child relation.

Existence Dependencies: The existence of a relationship (line) indicates that, should an instance of the child entity exist (at the "cardinality" end), the related parent must exist. An example of this dependency from the ARM is that: A Process Step instance cannot exist unless an Assembly Occurrence exists.

Identification Dependencies: In addition to the existence dependency above, if the relationship is a solid line, the unique identification of the child requires the key of the parent in addition to the key declared in the child. An example is the Process Step can only be uniquely identified by knowing the Assembly Occurrence to which it "belongs."

Round corners are used on an entity-boxes to indicate that there exists a parent entity which provides some of the key for identification. For entity-boxs with square corners, all of the entity's key is owned by the entity; the entity exists independently within the given domain of the model.

\subsubsection{ARM Diagrams}

The ARM is intended to be an enterprise-view of the information about the LEP as a product. The 


\section{Information Requirements}

ARM serves as a reference-point for the data implementation as captured in drawings, in the CAD system, and specified for IGES exchange in the next section.The ARM diagrams (views) constitute an information model that documents the information structures needed to support the activities in the Application Activity Model (AAM) and to develop the corresponding IGES Application Interpreted Model (AIM).

Product Types or "technologies" associated to the ARM Entities
a) Integrated Circuits
b) Hybrid Microcircuit Assemblies (or Micro-chip Modules)
c) Printed Circuit Assemblies
d) Flex (FLEX) Circuits
e) Flexible (Flex) Cables 


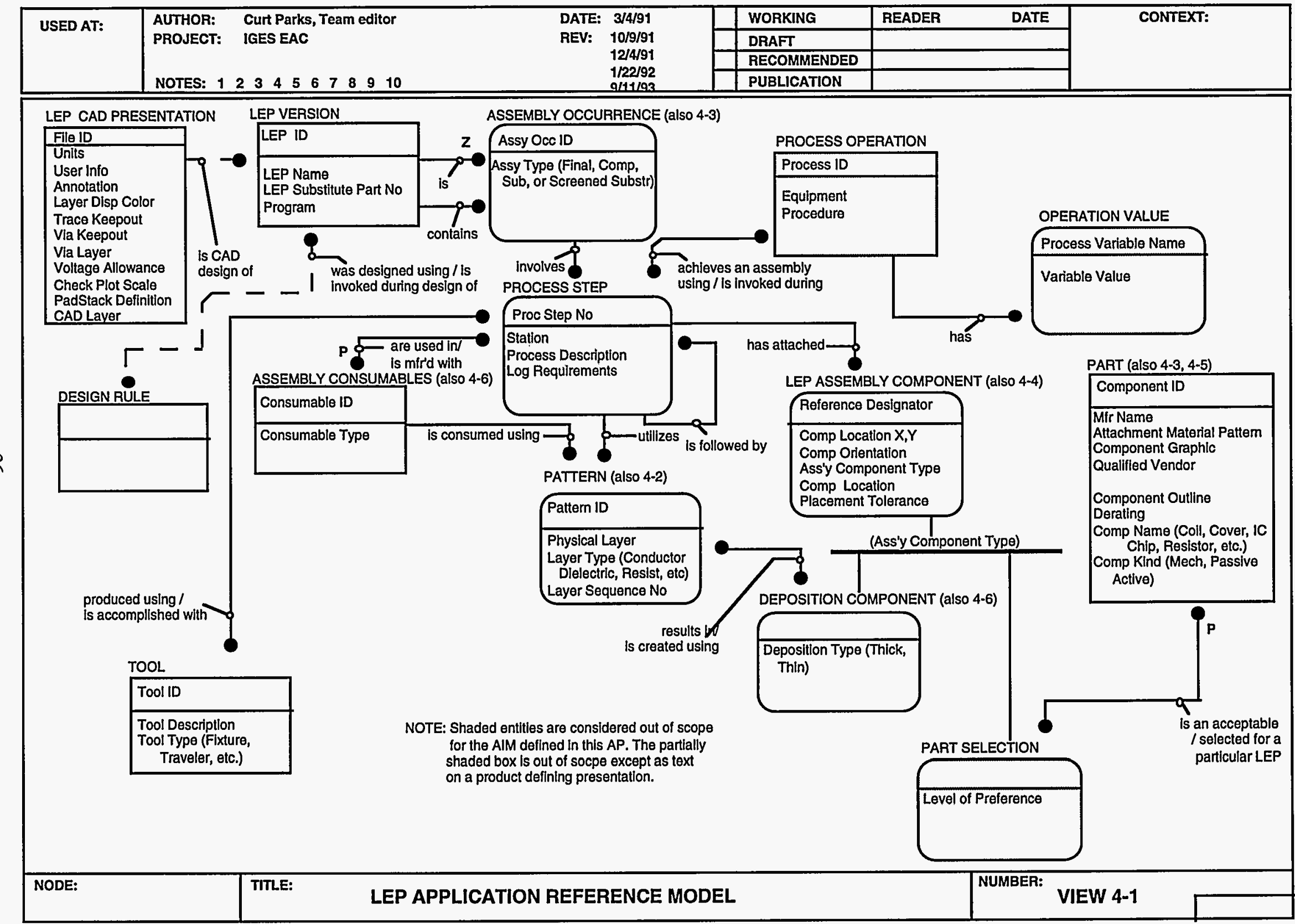




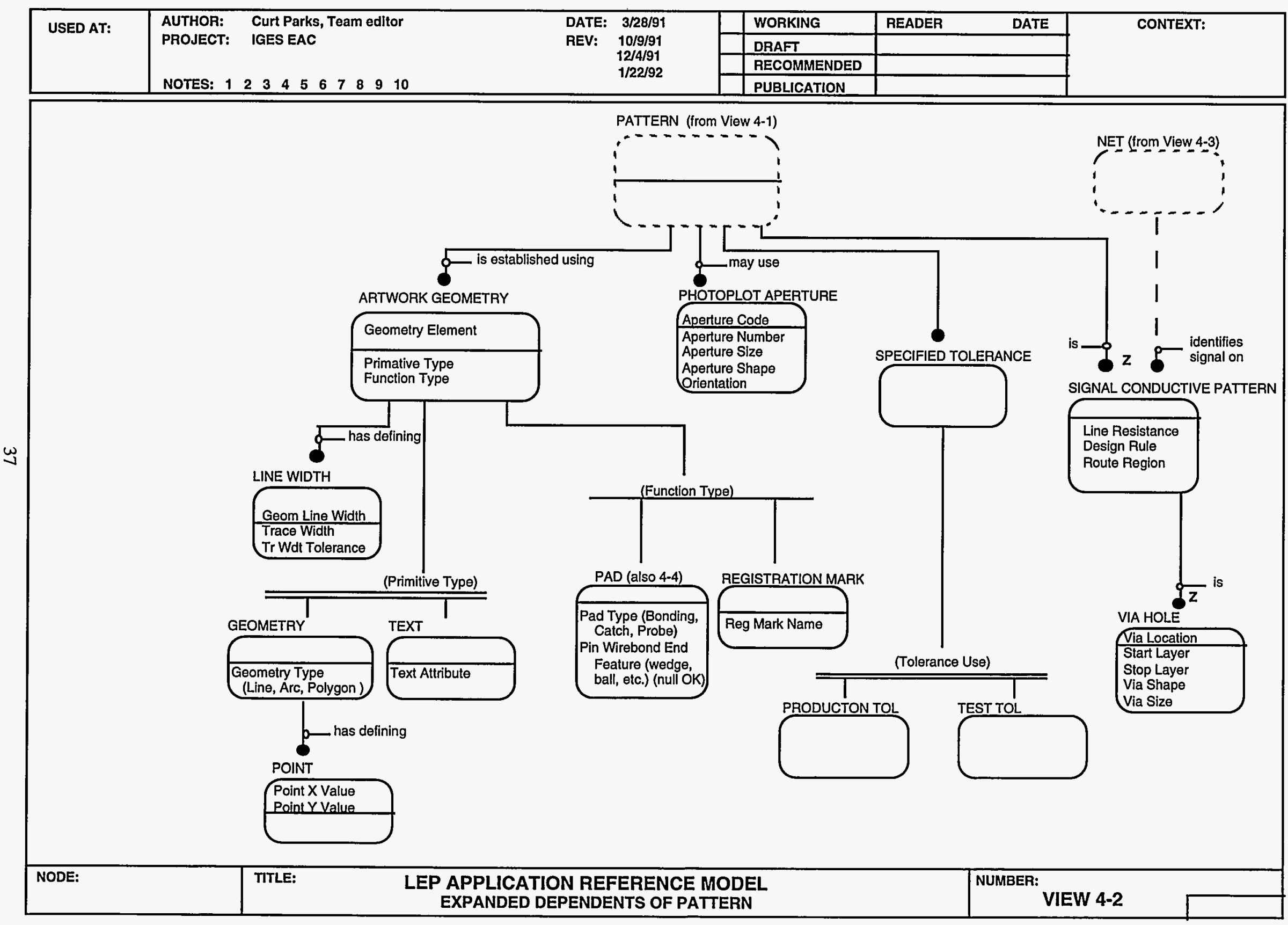




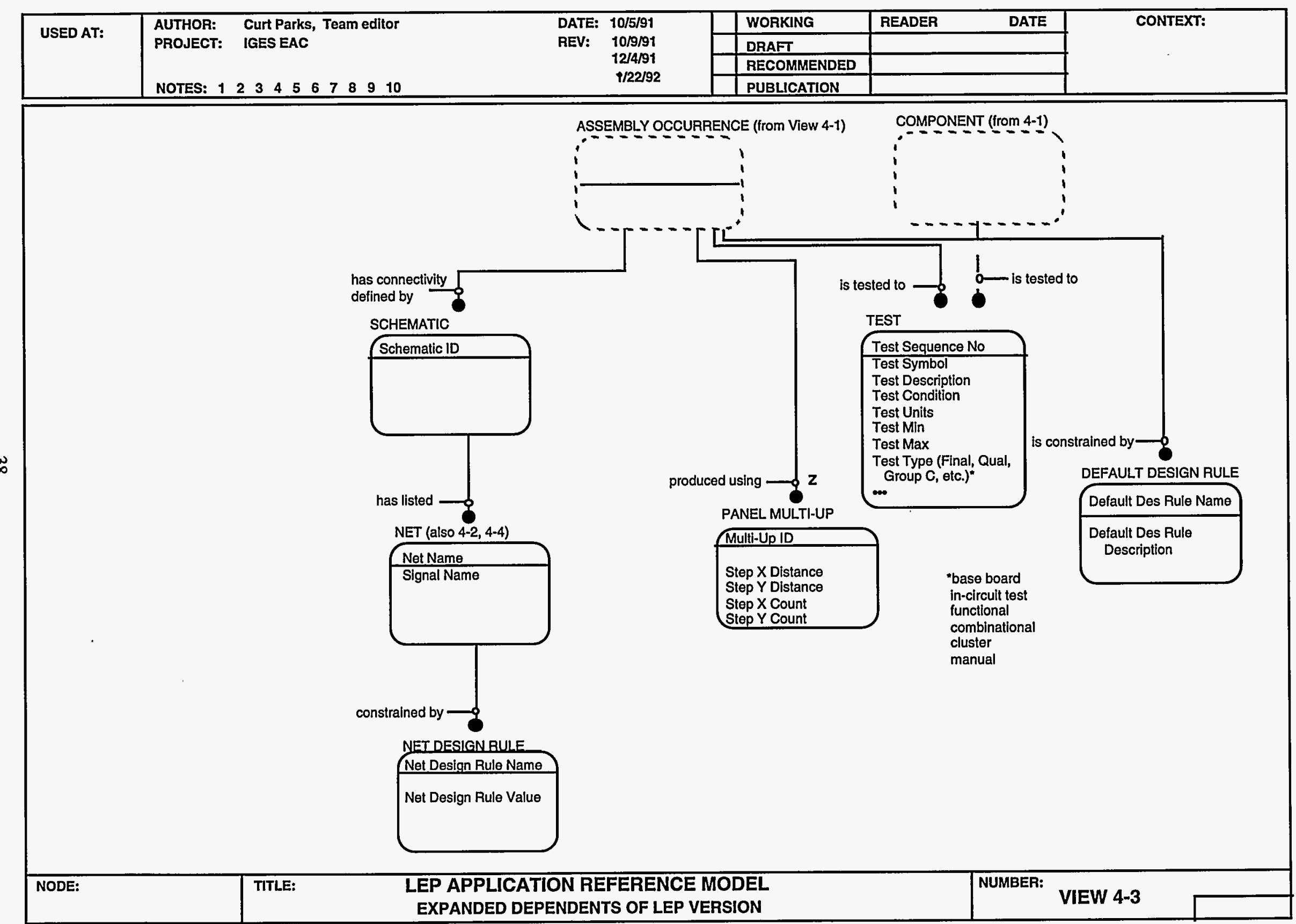




\begin{tabular}{|c|c|c|c|c|c|c|c|c|}
\hline \multirow{4}{*}{ USED AT: } & \multirow{3}{*}{\multicolumn{2}{|c|}{$\begin{array}{ll}\text { AUTHOR: } & \text { Curt Parks, Team editor } \\
\text { PROJECT: } & \text { IGES EAC }\end{array}$}} & \multirow{4}{*}{\multicolumn{2}{|c|}{$\begin{array}{ll}\text { DATE: } & 10 / 5 / 91 \\
\text { REV: } & 12 / 4 / 91 \\
& 9 / 15 / 93\end{array}$}} & WORKING & READER & DATE & \multirow[t]{4}{*}{ CONTEXT: } \\
\hline & & & & & DRAFT & & & \\
\hline & & & & & RECOMMENDED & & & \\
\hline & NOTES: & 0010 & & & 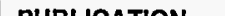 & & & \\
\hline
\end{tabular}

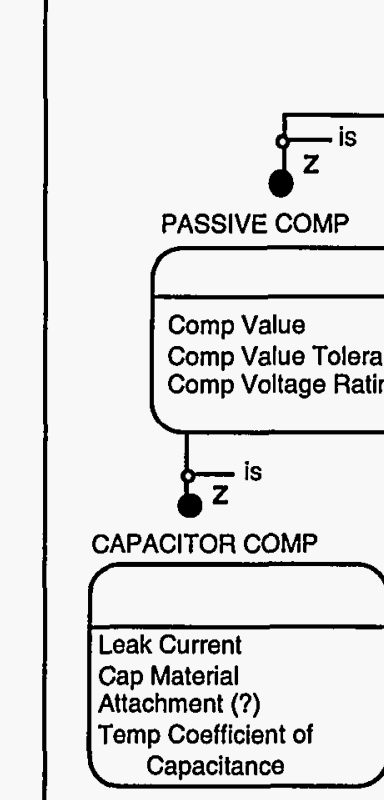

LEP ASSEMBLY COMPONENTS (from View 4-1)

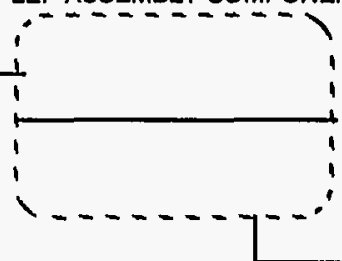

NET (trom View 4-3)
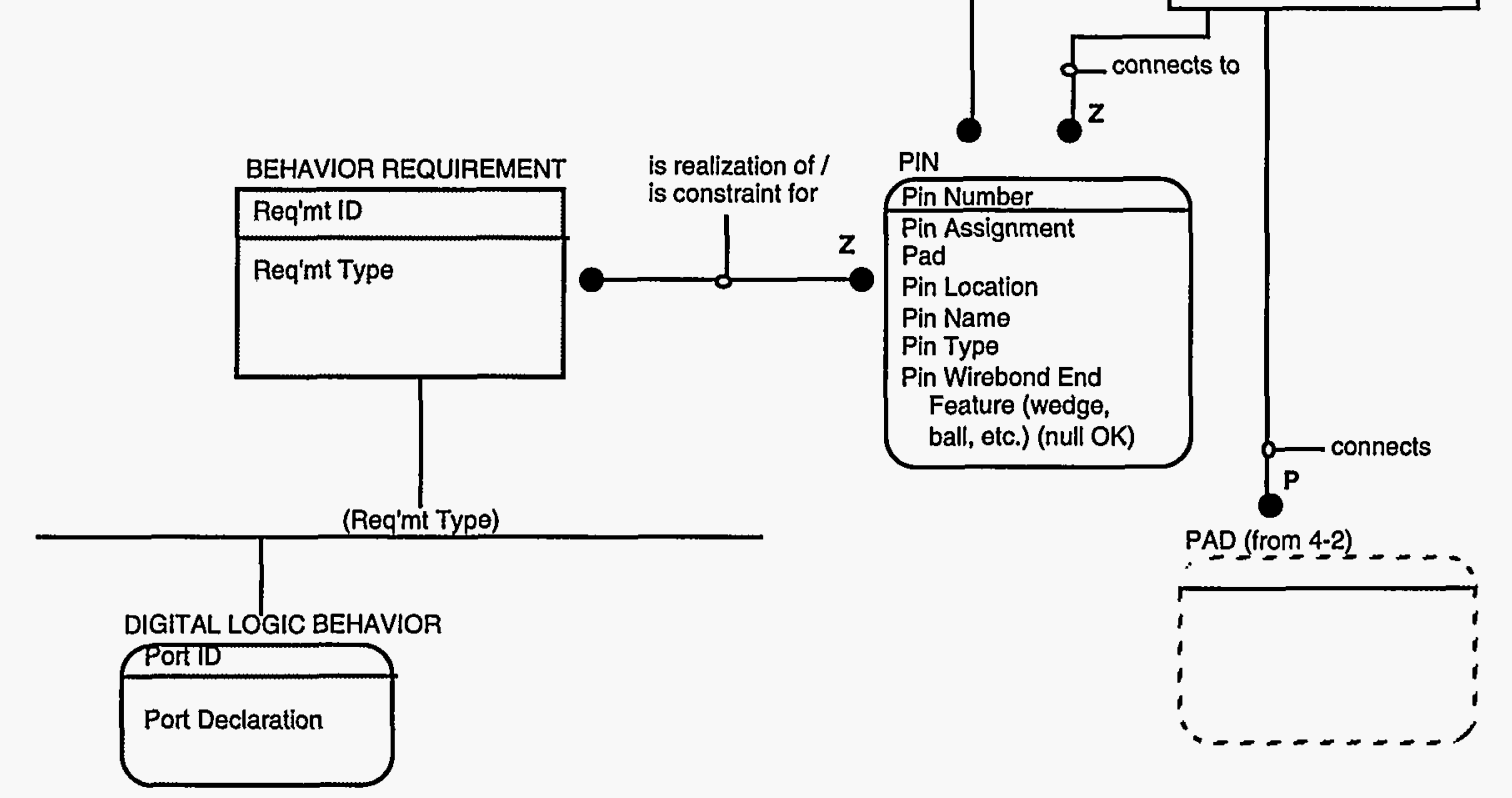

PIN

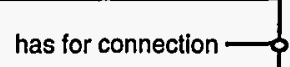

identifies

(also 4-6)

Wirebond Sequence No

Wirebond Min Length

Wirebond Max Length

Wire Size 


\begin{tabular}{|c|c|c|c|c|c|c|}
\hline \multirow{4}{*}{ USED AT: } & \multirow{4}{*}{$\begin{array}{l}\begin{array}{l}\text { AUTHOR: } \\
\text { PROJECT: }\end{array} \\
\text { IGES EAC } \\
\text { NOTES: } 1 \begin{array}{lllllllll}1 & 2 & 4 & 5 & 6 & 7 & 8 & 9 & 10 \\
\end{array}\end{array}$} & \multirow{4}{*}{ 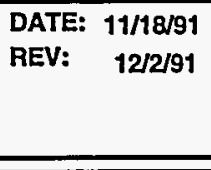 } & $x$ & WORKING & READER & \multirow[t]{4}{*}{ CONTEXT: } \\
\hline & & & & DRAFT & & \\
\hline & & & & RECOMMENDED & & \\
\hline & & & & PUBLICATION & & \\
\hline
\end{tabular}

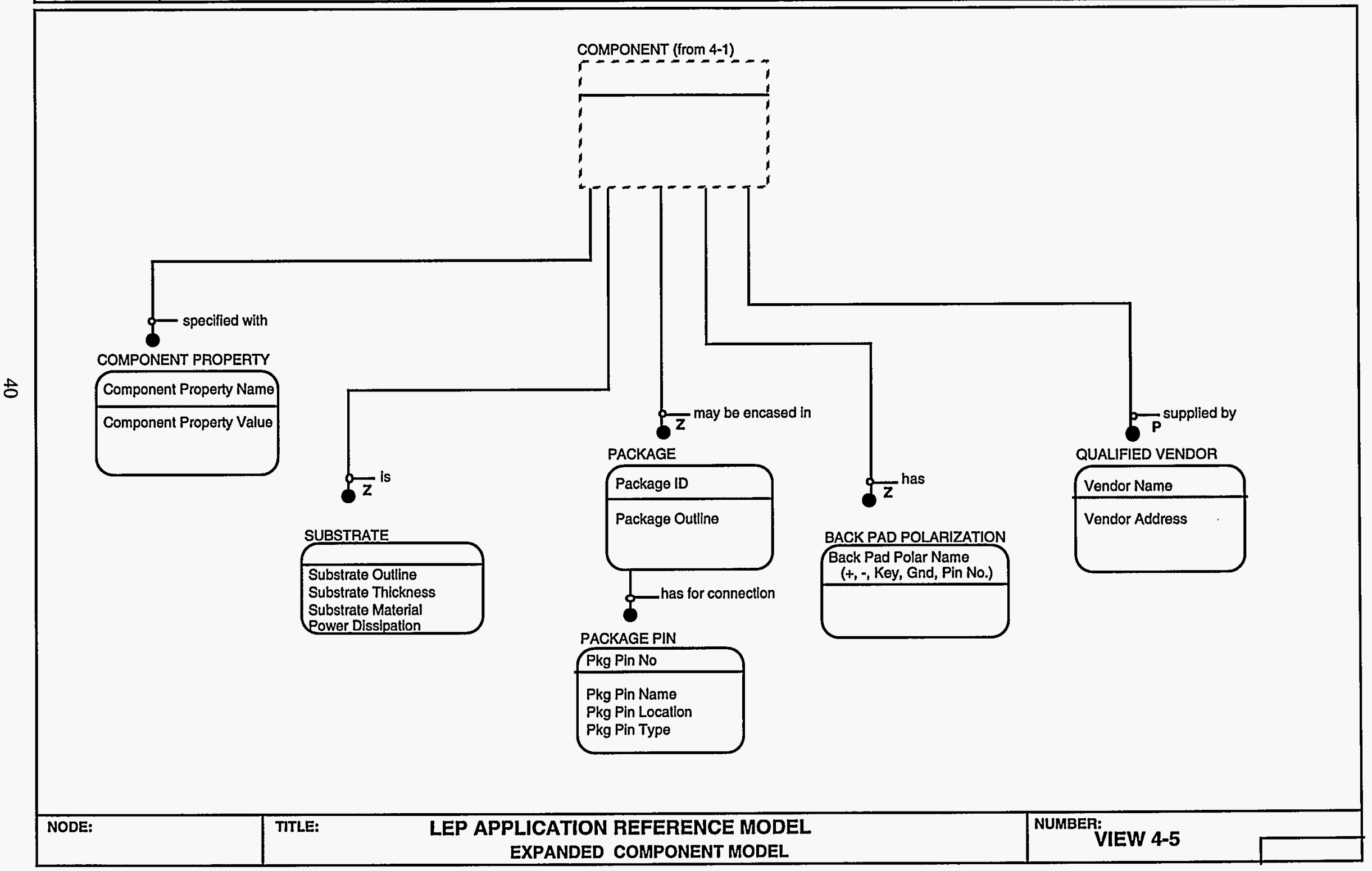




\begin{tabular}{|c|c|c|c|c|c|c|c|}
\hline \multirow{4}{*}{ USED AT: } & \multirow{4}{*}{\multicolumn{2}{|c|}{$\begin{array}{l}\begin{array}{l}\text { AUTHOR: } \\
\text { PROJECT: }\end{array} \begin{array}{l}\text { Curt Parks, Team editor } \\
\text { IGES EAC }\end{array} \\
\text { NOTES: } \begin{array}{llllllllll}1 & 2 & 3 & 4 & 5 & 6 & 7 & 8 & 9 & 10\end{array}\end{array}$}} & \multirow{4}{*}{$\begin{array}{ll}\text { DATE: } & 10 / 5 / 91 \\
\text { REV: } & 10 / 9 / 91 \\
& 11 / 29 / 91 \\
& 122 / 91 \\
& 1 / 14 / 92\end{array}$} & WORKING & READER & DATE & \multirow[t]{4}{*}{ CONTEXT } \\
\hline & & & & DRAFT & & & \\
\hline & & & & RECOMMENDED & & & \\
\hline & & & & - & & & \\
\hline
\end{tabular}

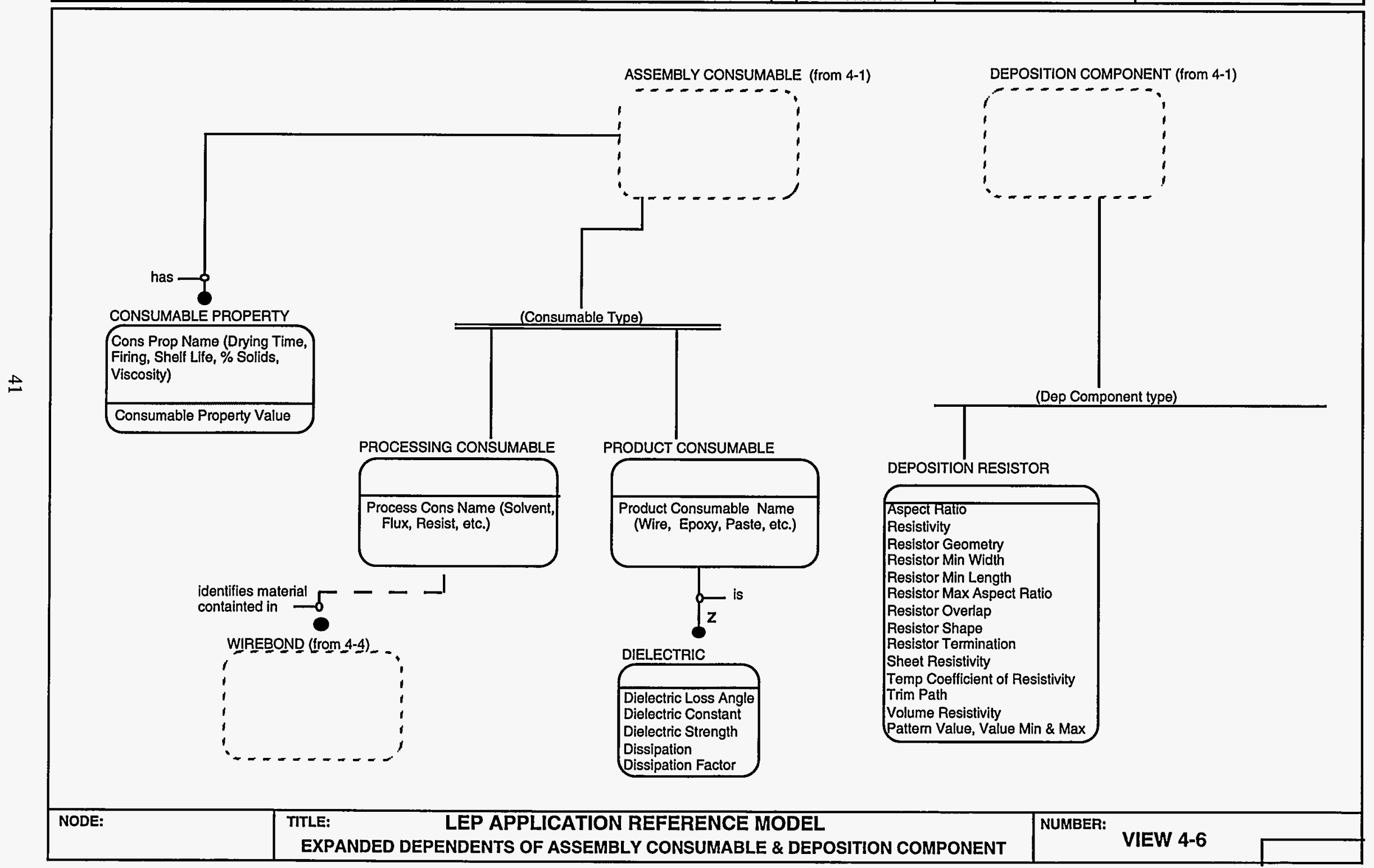


Information Requirements

\section{Blank Page}




\section{IGES APPLICATION INTERPRETED MODEL}

The Application Interpreted Model (AIM) is an information model that describes the logical information structures required for accomplishing a physical implementation of an associated application reference model. This AIM is prepared at a level of abstraction that is sufficient for selecting the necessary IGES ${ }^{7}$ entities for an application protocol.

The scope of this AIM is currently limited to LEP representation information contained in computer-aided design (CAD or CAE) systems. These systems are in widespread use and translation between dissimilar systems is a high priority in the user community. IGES serves as the implementation for this information because many CAE suppliers are familiar with the specification and support it in their software packages.

A broader scope for this AP that includes manufacturing, test, simulation, behavior and documentation is desired by users and manufacturers of LEPs. Much of this information is outside the scope (see also Abstract) of most CAE systems. Some of this information is beyond the scope of the IGES specification.

The graphical constructs of the AIM are presented in section 5.2 followed by the AIM diagrams in section 5.3. The AIM diagrams are divided into subsections as follows:

\subsubsection{Interface}

5.3.2 LEP Specific

5.3.3 Display Geometry

5.3 4 Miscellaneous

5.3.5 DE Referenced

5.3.6 DE Section

Interface object models are defined in this section. Each interface object model represents a "view" or "perspective" of an LEP in which one may exchange data. The interface object models describe the set of independent entities (DE Attribute - Subordination Status equals 0) that are in an IGES file which describe some aspect of an LEP. There are currently three interface objects; LEP Part Library IGES File, LEP Physical Layout IGES File and LEP Technical Mlustration IGES File. Future interface objects might be LEP Schematic IGES File or LEP Manufacturing IGES File.

This section defines objects that are unique and specific to LEPs.

This section defines objects that are common to any $\mathrm{CAE} / \mathrm{CAD} / \mathrm{CAM}$ system that utilizes 2-dimensional geometry.

This section defines subordinate objects which, when used in the indicated combination, represent an LEP specific object.

This section defines objects that are only referenced from the DE section of another object.

This section defines value objects that represent pre-defined $\mathrm{DE}$ section values.

In order to conform to this AIM an CAE system shall be capable of reading and writing files that conform to the entire LEP PHYSICAL LAYOUT IGES File (see 5.3.1.2) object. It is appropriate to support all objects, but it is not necessary in terms of conformance.

\footnotetext{
7. This section depends on the IGES document cited in section 2 for definitions and explanations of the for-
} mat structure and the entities cited. 


\subsection{ARM to AIM Mapping}

The ARM found in section 4 is an information model that documents the user view of the information structures of the subject application and provides the baseline from which candidate interpreted models are developed. For an IGES AP, the ARM is then used to develop the corresponding IGES Application Interpreted Model (AIM). The AIM shows how the information content from the ARM is to be expressed by a subset of IGES entities. Annotated outside the AIM object boxes (for reference) are the ARM entities which provided the requirement. (Note: The reader should not infer a one-to-one mapping; the AIM has a finer level of detail than the ARM.)

The PDQ (Product Data by Queries) IGES file represents the final product, fully assembled, with both electrical and mechanical parts installed. It has tooling holes, fiducials, and all the other PWB features needed by the product processes. This PDQ file serves information needs from conceptual design through packaging, shipping, and maintenance. In addition to being physically complete, the file also contains a significant amount of non-graphic information, including connectivity/ netlist information, component values, traces, flows, properties, part numbers, and more.

The IGES entities selected for use in the AP specification should be selected to best convey the information needed in subsequent processes subject to constraints defined in this section. The options for the use of the entities within this subset have been restricted so that only one method is available for carrying each element of information from the ARM. The set of IGES entities and the necessary restrictions on the Global, Directory Entry, and Parameter Data Section field values have been developed by using the ARM and the AIM.

\subsection{IGES Structure and Syntax of the Application Interpreted Model}

Some graphical objects will be defined in this section to correspond closely to the constructs of the IGES file structure.

\subsubsection{IGES File Structure}

The following is the brief review of the IGES file structure from a predecessor ${ }^{8}$ to this document: "IGES provides format guidance for data transfer/archive. It defines several data structures or entities, whose pre-defined purpose may be geometry, annotation, or structure. The individual vendor can then map information contained in his proprietary database into this neutral format, then transfer that information to other vendor's applications, or archive the file for future use. IGES is fully three dimensional, and includes finite element, graphics, documentation/annotation, manufacturing and electrical entities.

... The transfer mode will commonly be character (ASCII) ... The logical record length is 80 characters (block to 800 on tape) and is formatted for human readability and interaction. The advantage of the ASCI format are at the sacrifice of file size. However, there are standard file compression algorithms which may be used to achieve great reductions in file size, but the compressed files must be decompressed prior to translation. Also see ANSI X3.27-1978 labeled variable block.

The IGES data file structure consists of five sections. The start section, contains $72 \mathrm{col}-$ umns of user comments which are not to be processed by the program other than listing to

\footnotetext{
8. see Appendix E of [11].
} 
screen or printer. Next is the global section, a free format area specifying certain file attributes such as creation date, time, location, designer, units, display resolution, etc. The third section is the Directory Entry (DE) section containing fixed format attribute data (level, pen, translation, view, etc.) for each entity. The Parameter Data (PD) section follows and contains actual parameters for each of the entities in the DE section. Finally, the terminate section contains a single record containing the count of the records in each section. The entities come in two classes, those that are pre-defined and documented by the IGES committee (numbers 0001 to 5000), and those that are implementor definable (5001 to 9999). The constructs for a valid IGES file shall conform to those specified in the current version of the IGES Specification."

\subsubsection{START Section}

The IGES file Start section shall be in accordance with MLL-D-28000A.

\subsubsection{TERMINATE Section}

The IGES file Terminate section shall be in accordance with the IGES document identified in Global parameter number 23 .

\subsubsection{GLOBAL Section}

The IGES file Gobal section shall be in accordance with the IGES document identified in Global parameter number 23. Further information on particular global parameters are described in Section 5.2.2 below.

\subsubsection{DIRECTORY ENTRY and PARAMETER DATA Sections}

The IGES file Directory Entry and Parameter sections shall be in accordance with IGES version 5.2 or later. The values applicable to these section entries are dependent on the IGES entities and the objects (information structures) contained in the file. Those entities and objects shall be structured as defined in the remainder of this section.

\subsubsection{Global Values and Entities List}

The following are IGES constructs that are used to fulfill the requirements of the AIM (View 4) for LEP technology.: The IGES entities have been selected to ensure the constructs uniquely attributable to an LEP will be correctly transferred when they are postprocessed. Other entities defined in the IGES Specification may be used for other purposes, however, these other entities should not be used for the purposes stated in the AIM. Parenthetical statements that follow the entities guide the reader to the relationships defined in the AIM. The IGES Specification should be consulted for the details of the syntactical constructs needed to produce valid IGES files.

Globals: (these are used to identify general product-file attributes)

Global No. Description

Product identification from sending system

$4 \quad$ File name

5 System identification 
$13 \quad$ Model space scale

$14 \quad$ Unit flag

15 Units

$21 \quad$ Name of author

22 Author's organization

23 IGES Version Code number

25 Date and time the model was created or last modified

\section{IGES Entities used in the AIM:}

The AIM defines objects needed by the designer of translators and other software. The information requirements for these objects are defined within the ARM. The ARM information elements, excepting those identified as "out of scope," have been developed as detailed IGES-specific objects in the AIM.

All versions of IGES subsequent to version 3.0 are capable of transfering LEP information. To provide guidance needed to interpret files written to various versions, this section contains information on each applicable IGES entity; the initial version for the entity, and the existence of committee work which may extend or define its functionality. The following categories for IGES documented entities are utilized within this AIM. Individual object definition models in the AIM contain usage restrictions (i.e., usage constraints) appropriate to the application. Please refer to the applicable IGES document for detailed information regarding these entities. The "Version" column specifies the status of the entity. Applicable values are:

Vn The entity is valid in this and subsequent versions and is not expected to be modified to be used within this AIM.

Gray The entity currently exists and does not need to be modified to be used within this AIM. The entity is approved, but may not have been verified through testing.

$+\quad$ The entity either has proposed extensions and is being considered by the Electrical Applications Committee or has been submitted to the IGES Project for ballot. The number in parenthesis following the " + " relates to the "Request for Change" (RFC) document defining the new attributes proposed for the entity.

WIP Work in Progress. The entity has been submitted to the Electrical Applications Committee for consideration; it is not a valid entity for this AP version, however exchange of data by agreement between parties is encouraged. Each of these entities may be written into a "by agreement" file as a user-defined entity to be numbered in the range of 5000 to 9999 . The last three numbers indicated for the entity relate to the "Request for Change" which defines the attributes proposed for the entity.

Please contact either of the editors for a copy of a cited Request for Change of interest. Implementors are encouraged to return results from implementation and exchange through the use of the constructs defined in an RFC. Implementors are also encouraged to write an RFC where user- 
defined IGES entities have demonstrated a capability.

\begin{tabular}{|c|c|c|c|}
\hline Version & Type & Form & Description \\
\hline V1.0 & 100 & 0 & Circular Arc \\
\hline V1.0 & 102 & 0 & Composite Curve \\
\hline V1.0 & 106 & 11 & Copious Data - Planar Piecewise Linear Curve \\
\hline V2.0 & 106 & 63 & Copious Data - Simple Closed Planar Curve \\
\hline V1.0 & 110 & 0 & Line \\
\hline V1.0 & 116 & 0 & Point \\
\hline V1.0 & 124 & $0-1$ & Transformation Matrix \\
\hline V2.0 & 125 & $0-4$ & Flash \\
\hline V3.0 & 132 & 0 & Connect Point \\
\hline V3.0 & 212 & $0,1,6,7 \& 8$ & General Note \\
\hline V4.0 & 230 & 0 & Sectioned Area (used as filled region) \\
\hline V1.0 & 308 & 0 & Subfigure Definition \\
\hline V3.0 & 312 & 1 & Text Display Template \\
\hline V3.0 & 314 & 0 & Color Definition \\
\hline V3.0 & 320 & 0 & Network Subfigure Definition \\
\hline V1.0 & 402 & 1 & Group - Unordered With Back Pointers \\
\hline V3.0 & 402 & 14 & Group - Ordered With Back Pointers \\
\hline$V 3.0+(544)$ & 402 & 18 & Flow Associativity \\
\hline V1.0 & 406 & 1 & Property - Definition Levels \\
\hline $\mathrm{V} 2.0+(538)$ & 406 & 2 & Property - Region Restriction \\
\hline$V 2.0+(545)$ & 406 & 5 & Property - Line Widening \\
\hline $\mathrm{V} 2.0$ & 406 & 9 & Property - Part Number \\
\hline V3.0 & 406 & 15 & Property - Name \\
\hline $\mathrm{V} 2.0$ & 406 & 10 & Property - Hierarchy \\
\hline $\mathrm{V} 5.0+(537)$ & 406 & 24 & Property - Level to LEP Layer Map \\
\hline V5.0+(543) & 406 & 26 & Property - LEP Substrate Hole Property \\
\hline V5.1, Grey & 406 & 27 & Property - Generic Data \\
\hline WIP & 406 & 27 & Property - LEP Component Placement Boundary \\
\hline WIP & 406 & 27 & Property - LEP Physical Layer Description \\
\hline V1.0 & 408 & 0 & Subfigure Instance \\
\hline WIP(513) & 408 & 1 & Modified Subfigure Instance (under revision) \\
\hline V3.0 & 420 & 0 & Network Subfigure Instance \\
\hline
\end{tabular}

Some of the AIM objects are specific to a particular product type. These are listed below.

Printed Circuit Board Objects

DRILLED HOLE 


\section{LEP Constructs Supporting Signal Conductivity}

Traces Traces use simple geometry objects such as LINES and ARCS. They interconnect electronic components and carry signals. Physically, they have a specific width and may pass between the different layers by means of vias and through-pins.

Jumpers A physical interconnect which may be implemented as a component or a trace, but which will have only one signal name.

Planes Planes, such as ground and power planes, outline the appropriate (filled) area.

Vias Vias are holes or voids made between two or more layers on the LEP. They are plated so they can carry the signal. The holes are made by different means such as drilling, milling or cutting. This variability makes it hard to select specific entities which relate to some aspects of vias. However one makes the hole or void, the functions of the via are still the same. Vias may be through, blind, or buried. Vias are often used as test points.

Pad stacks (through and SMT) Pad stacks provide the electrical interface between the LEP substrate and the component. Pad stacks can be either through_hole or surface mount.

\section{LEP Constructs Supporting Components}

Network Subfigure Definition The Network Subfigure Definition (Type 320) defines an electrical component. It has specific points where connections between it and a signal carrier can be made. These points are component terminals of the LEP. The Connect Point Entity (Type 132) models the component terminal. They may be pins, pads, wire bonds, or any other means of connecting a component to a signal. In addtion to Connect Points (Type 132), the Network Subfigure Definition (Type 320) contains geometry defining functionality, such as thermal outline, footprint outline, and placement outline.

The Network Subfigure Definition (Type 320) may also contain properties, things like technology type (through-hole or surface mount), board side placement rules, value, tolerance, version number, and any special instructions. A Network Subfigure Instance (Type 420) places the electrical component defined by the Network Subfigure Definition (Type 320). The LEP AP requires each instance to have a corresponding definition within the same file. More than one Network Subfigure Instance (Type 420) in the file may reference the same Network Subfigure Definition (Type 320). Each Network Subfigure Instance (Type 420) is unique, it is placed at a specific location, and it has a unique reference designator. The instance uses the geometry and the properties defined in the defintion. The Network Subfigure Instance (Type 420) has one Connect Point (Type 132) for each pad/pin. Each has a counterpart in the Network Subfigure Definition (Type 320). The counterpart Connect Points (Type 132) must correspond because different signals connect to the same generic definition PIN at each instance. For example, both R1 and R2 may reference the Network Subfigure Definition (Type 320) having the name (axial.100). Distinct Connect Points (Type 132) in the instances are needed to connect R1 pin 1 to sig_1 while R2 pin 1 connects to ground.

Connect Point A Connect Point (Type 132) defines a specific and unique point of connection between two or more signal carriers. It provides a physical location, a function flag, and an identifier. The Connect Point (Type 132) references an ordinary Subfigure Definition (Type 308) which 
contains information such as pad size and shape, drill hole (if applicable), and display geometry. The most common examples of Connect Points are vias, test points, component pins, or SMT dedicated through-pins.

\section{LEP Constructs Supporting Connectivity}

Connectivity is represented by appropriate use of the Network Subfigure Definition (Type 320); the Network Subfigure Instance (Type 420); the Connect Point (Type 132); and the Flow Associativity (Type 402, Form 18). The Flow Associativity (Type 402, Form 18) may include connecting geometry.

The Flow Associativity provides the signal name and pointers to all features associated with that signal. Such features include pins, pads, stand-alone vias, traces and planes. There is one Flow Associativity (Type 420 , Form 18) for each signal in the file. Note that it is possible to include both the logical and physical product data in one file using the Flow "Type" flag or entity level assignments to discriminate between the two product representations. Though permitted by IGES, having both logical connectivity and physical connectivity in the same file is not conformant to the LEP AP.

"Backpointers" pointing from each of the features back to the Flow Associativity are not required for LEP AP conformance.

\section{LEP Constructs Supporting A Physical Representation}

A physical LEP is represented by simple closed planar GEOMETRY depicting its outer perimeter. An LEP may have more than one defined outline. There may be keepin/keepout outlines for component placement, traces, and vias, or tool path outlines, and other objects. All LEP outline geometry defining a specific function are logically required to be on the same IGES level. For example, the top layer traces of the actual LEP might be on level 1, the component keepin outline on level 2 , etc.

All entities on the LEP are subordinate to it. This includes: all components as Network Subfigure Instances (Type 420) and as Network Subfigure Definitions (Type 420), traces (JOIN), flow associativities (NET), padstacks (PADSTACK DEFINITION and PADSTACK INSTANCE), vias (VIA), tooling holes, fiducials, through holes, mounting holes, mounting hardware, and other objects. This means there must be a top level Network Subfigure Instance (Type 420) of the LEP which calls the top level Network Subfigure Definition (Type 320) of the LEP. If the top level instance is not present, nothing will be displayed.

What then is not subordinate to the LEP? Dimensions are not. Any assembly information, views/ draws, scales, grids, and certain kinds of details and cross-sections are not subordinate to the LEP representation.

Several applications require a PDQ LEP representation. This refers to one in which Product Data may be Queried. Computer controlled equipment can then extract needed data without manual intervention. For example, component insertion equipment can put a component at the $\mathrm{X}, \mathrm{Y}$ location specified by the component's Network Subfigure Instance. Operations which modify the physical representation of the LEP, such as scaling or rotation, will have adverse affects on some application systems. Constructs which are beyond the scope of an automated operation, such as 
dimensions and view/draw could stall or disable the processing system.

\subsubsection{Basic Syntax used in the AIM}

A graphic notation has been adopted to describe the IGES data structures.The objective of these diagrams is the ease of developing unambiguous IGES translators and proof-reading files. The notation is not intended for use as a conceptual modeling language. The brief descriptions which follow explain the use of principal elements of the notation; the Object Definition Block, the Object Instance Block, the Object Value Block, and the Cardinality code.

\subsubsection{Object Definition Block}

The Object Definition Block is used to depict an IGES entity type, form, directory entry values, parameter data values, and relationships to other IGES entities. There are several forms of the Object Definition Block depending upon how the object is being used within the model.

Each Object is depicted at least once using the Object Definition Block.

The graphic in Figure 5-1 is used to depict data and relationships to other objects for a particular object (usually an Application Information Requirement). The graphic shows the field name and required value(s) - if appropriate-for a particular field within the definition or instance of an object. A Value Block (see 5.2.3.3) may be referenced by either an Object Definition Block or an Object Instance block.

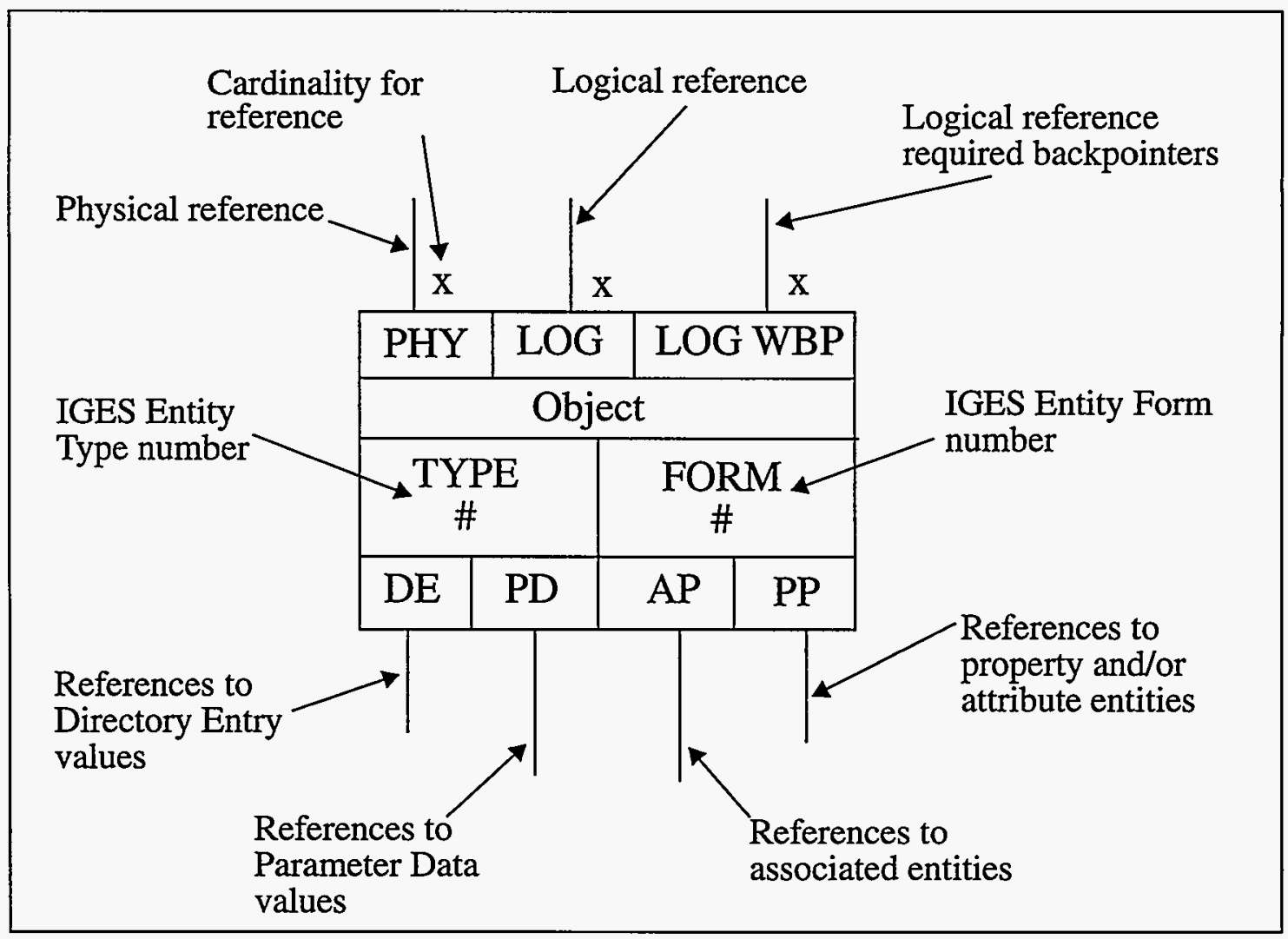

Figure 5-1. IGES Object and Referencing. 


\subsubsection{Object Instance Block}

The graphic in Figure 5-2 illustrates a particular reference to a previously defined object. The long form is available if the particular instance needs to reference property, attributes, and/or associativity entities.

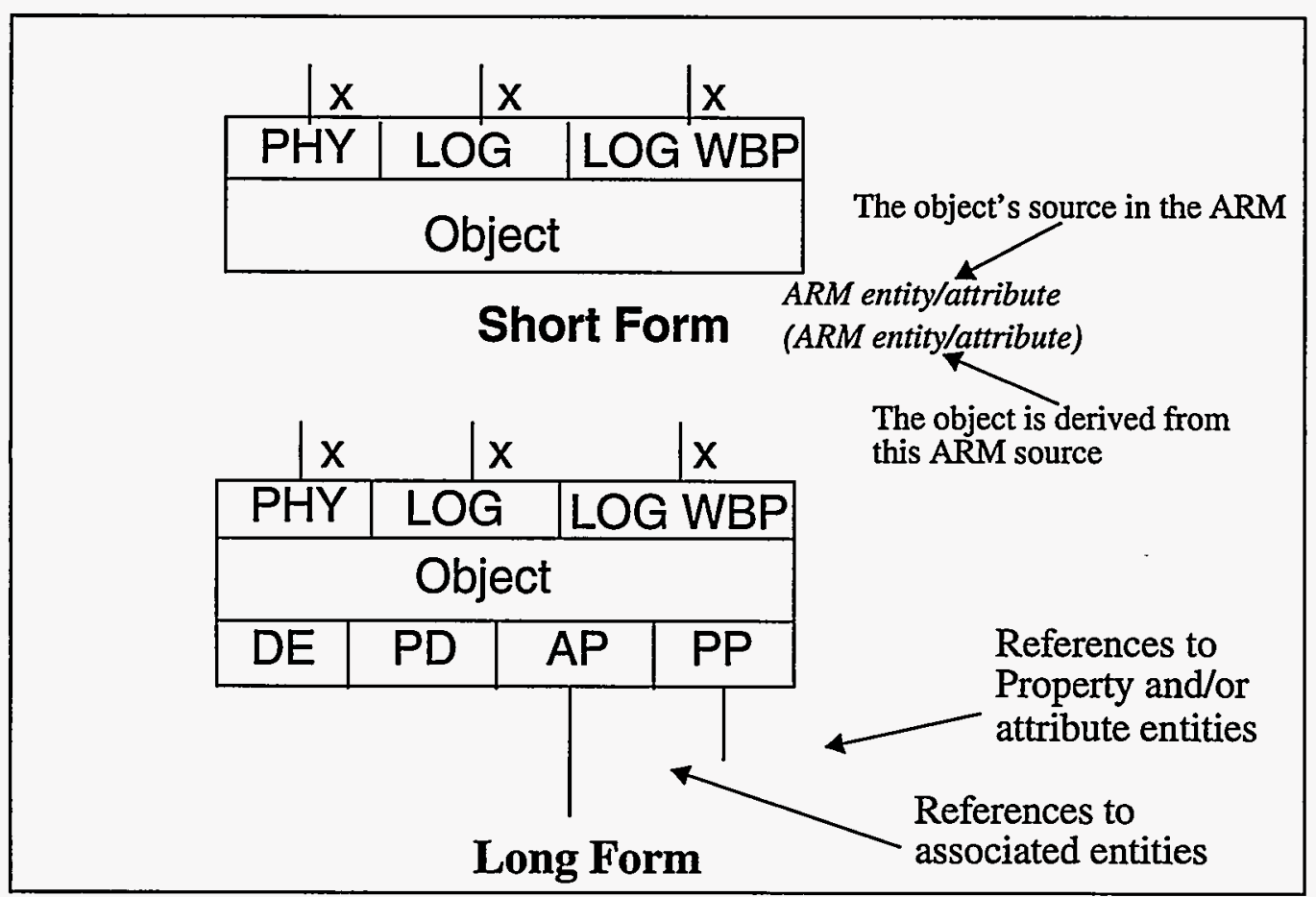

Figure 5-2. References to Previously Defined Object.

\subsubsection{Object Value Block}

The graphic in Figure 5-3 illustrates the field name and required value(s), if appropriate, for a particular field within the definition or instance of an object. This Value Block shown corresponds to the IGES parameter data section.

The Value Definition Block is used to show a set of Object reference mechanisms. The mechanisms provide ways to show either Directory Entry (DE) values or Parameter Data (PD) values. These values may reference other IGES Entities via their DE identifying numbers.

A Value Block may be referenced by either an Object Definition Block or an Object Instance 
Block.

\begin{tabular}{|c|c|c|c|}
\hline \multicolumn{3}{|c|}{ Value } & \multirow{8}{*}{$\begin{array}{l}\text { Field value as appropriate } \\
\text { Any acceptable value } \\
\text { Pointer to a predefined Instance Block } \\
\text { Value or pointer to a predefined Object Instance Block } \\
\text { Value will be ignored }\end{array}$} \\
\hline Index & Name & Value & \\
\hline 1 & name1 & Value & \\
\hline 2 & name2 & $*$ & \\
\hline 3 & name3 & $\Rightarrow$ & \\
\hline 4 & name4 & value $\mid \Rightarrow$ & \\
\hline 5 & name5 & N/A & \\
\hline$\cdots$ & name... & $\ldots$ & \\
\hline
\end{tabular}

Figure 5-3. Object Field Value Restrictions.

\subsubsection{Object Reference Mechanism}

The Object Definition Block or the Object Instance Block each may reference subordinate /child objects. Each object block will distinguish a reference to a subordinate object by any of the following mechanisms:

1) a directory entry (DE) pointer

2) a parameter data (PD) pointer, or (when appropriate)

3) an associativity backpointer (AP) or

4) a property pointer (PP)

as shown in Figure 5-4.

The reference to the subordinate object may involve either of the two kinds of dependencies described in 2.2.4.3.9.2 of IGES. The three dependency mechanisms indicated in Figure 5-4 are:

1) physically subordinate (Physical),

2) logically subordinate (Logical), or

3) logically subordinate with backpointer (LogW/BP).

The backpointers for the third mechanism are never explicitly shown in the figures that follow. The representations of cardinality indicators are shown in Figure 5-5. 


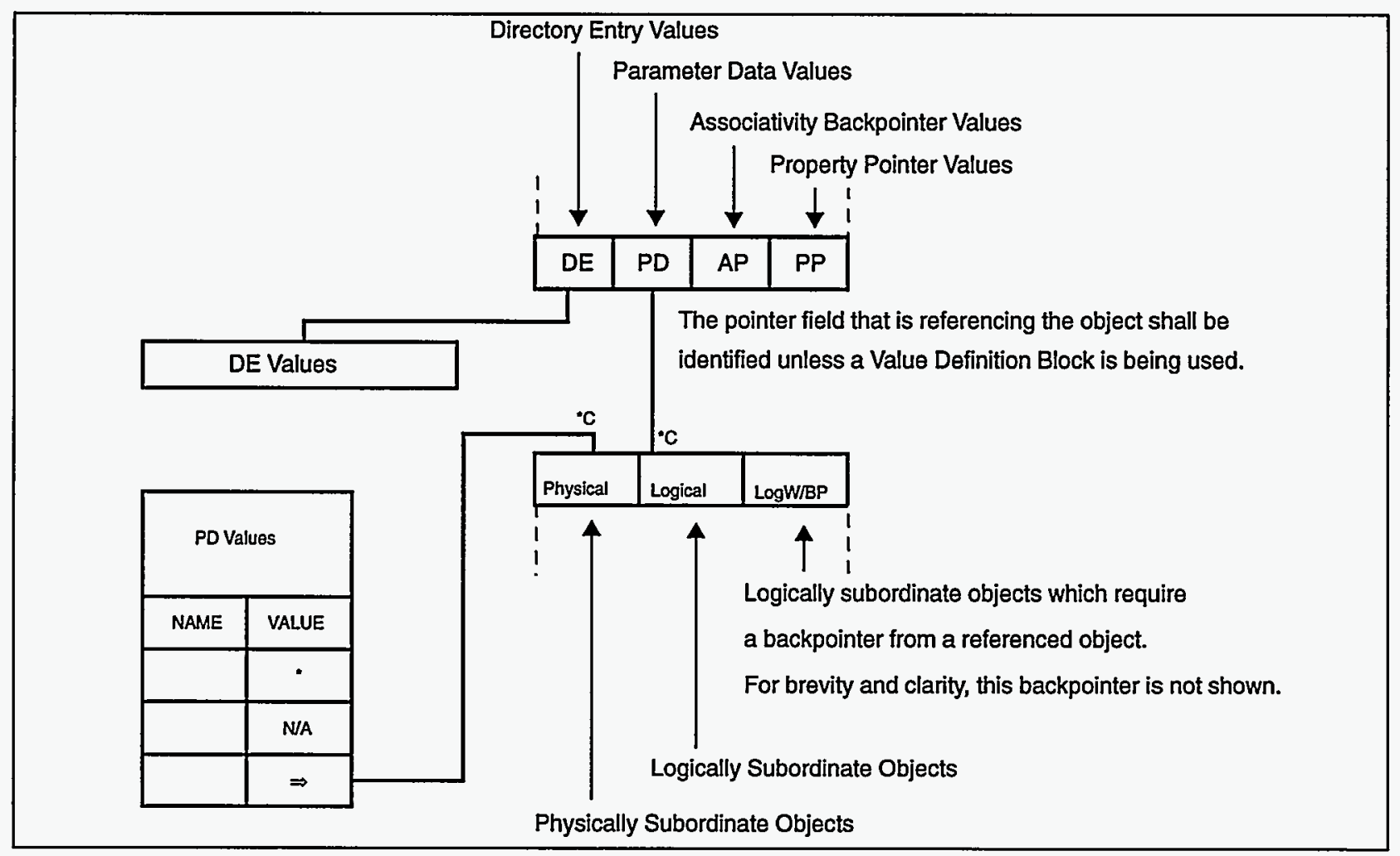

Figure 5-4. Reference Mechanism.

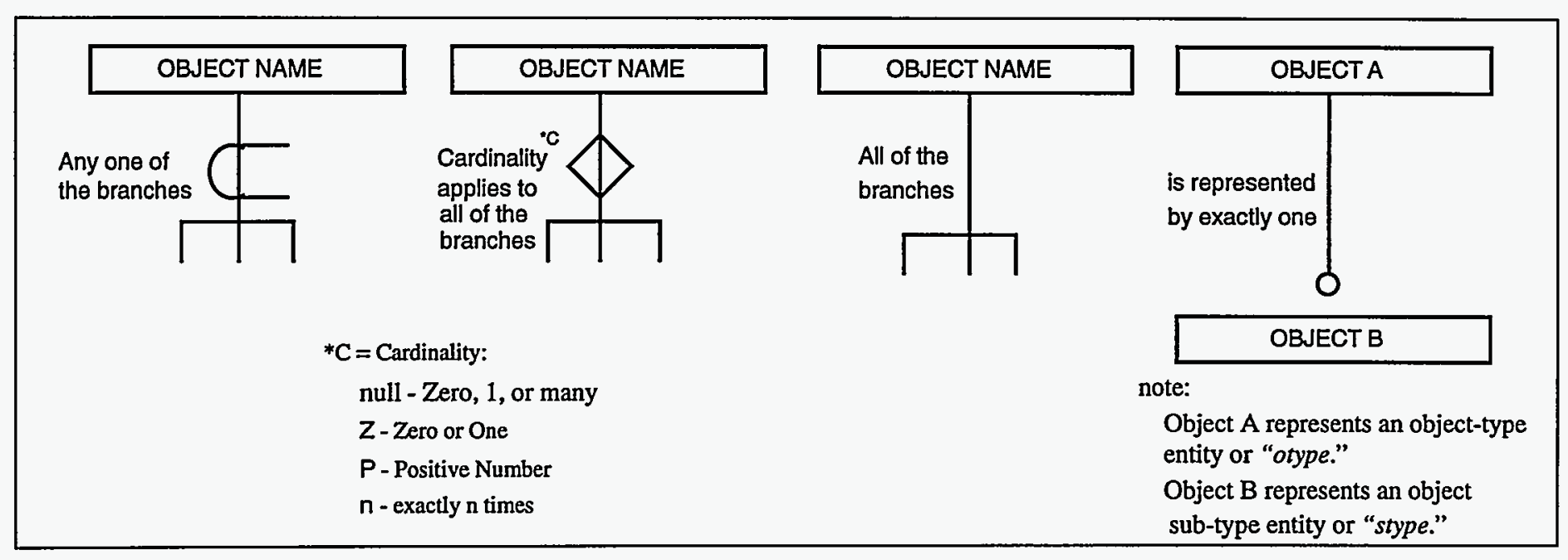

Figure 5-5. Representation Mechanism. 
Application Interpreted Model

BLANK PAGE 


\subsection{AIM Object Models}

Six categories of object models are documented below.

\subsubsection{Interface Object Models}

Three categories of object models have been defined for interfaces. Interfaces to a LEP part library IGES file, a LEP physical layout IGES file, and to a technical illustration IGES file are included.

\subsubsection{LEP Part Library IGES File}

\section{Description:}

The LEP PART LIBRARY IGES FILE object is an interu face model which enables computer-aided systems to write or read a library of LEP parts (e.g., Package Figure Definitions, Padstack Definitions, or Generic Part Definitions).

Requirements/Restrictions:

Translation Usage Notes:

General:

Output:

Input:

Please refer to IGES

\section{Section 3.6.4 for this object.}




\subsubsection{LEP Physical Layout IGES File}

\section{Description:}

The LEP PHYSICAL LAYOUT IGES FILe object is an interface model which enables computer aided systems to write or read LEP Physical Layout data.

\section{Requirements/Restrictions:}

1. When this object defines the layout of an LEP Panel, the GENERIC DATA PROPERTY which is referenced from the LEP INSTANCE object shall specify

$$
\text { panel_offset }
$$

\section{Translation Usage Notes:}

茨

\section{General:}

Existence of a Property Entity (Type 406, Form 27)

means something is there by default.

\section{Output:}

Input:

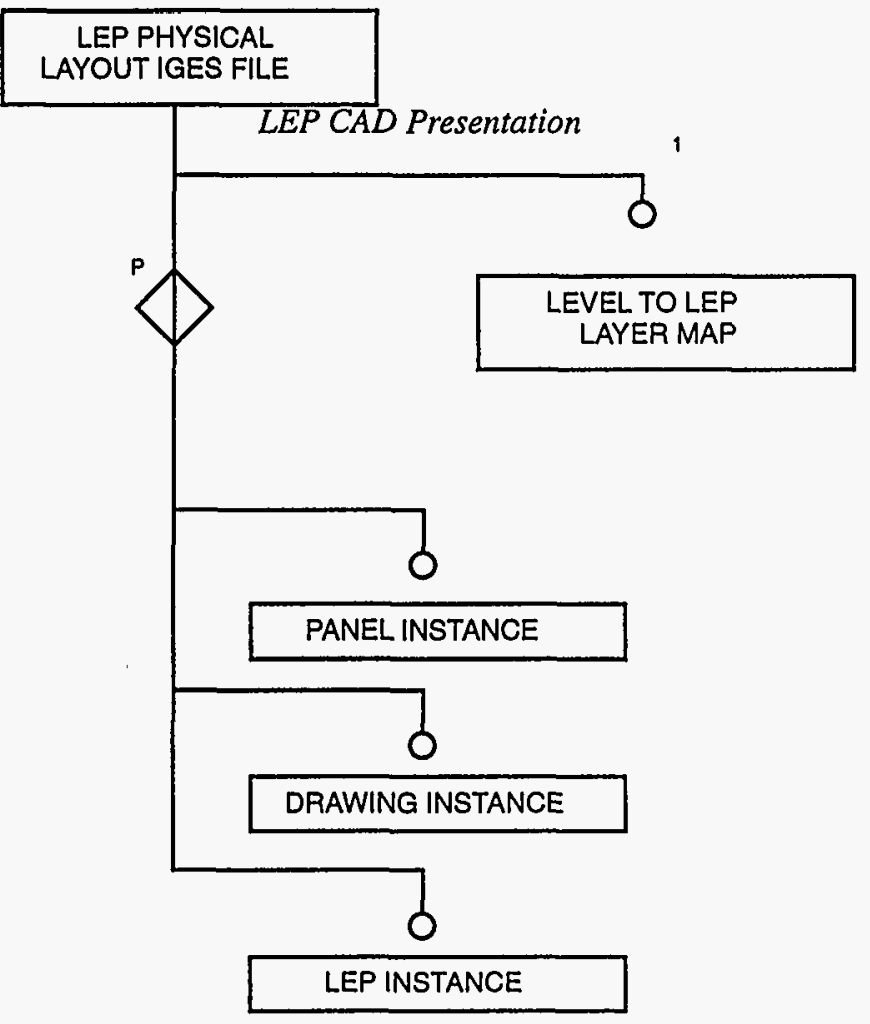




\subsubsection{LEP Technical Mlustration IGES File}

\section{Description:}

The LEP TECHNICAL ILLUSTRATION IGES FILE

object is an interface model which enables computeraided systems to write or read a graphic representation of LEP data. This interface model describes only geometry and annotation constructs.

\section{Requirements/Restrictions:}

Translation Usage Notes: y

General:

Output:

Input: 
LEP-Specific Object Models

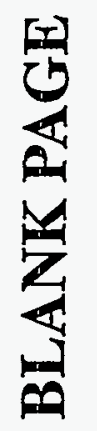




\subsubsection{LEP Property}

TheLEP PROPERTY object maps directly to the IGES Generic Property. Please refer to the IGES for additional information.

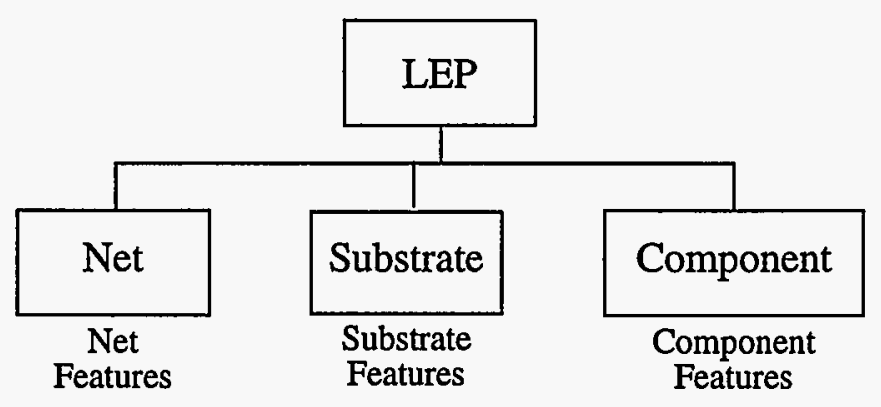

นั 1 . Net features include reference designator, pin number, signal name

2. Substrate features include outline, edge finger connectors, fiducial annotation (e.g. silk screen)

3. Component features include outline, PACKAGE SYMBOLDEFINITION and INSTANCE.
The following LEP object types and sub-types reference the LEP Property object:

5.3.1.2. LEP Physical IGES File

5.3.2.1. Component Keepin/Keepout

5.3.2.2. Drawing Definition

5.3.2.3. Drawing Instance

5.3.2.4. Generic Part Definition

5.3.2.5. Generic Part Instance

5.3.2.6. Hole

5.3.2.7. Join

5.3.2.13. LEP Definition

5.3.2.14. LEP Instance

5.3.2.17. Package Symbol Definition

5.3.2.18. Package Symbol Instance

5.3.2.19. Padstack Definition

5.3.2.20. Padstack Instance

5.3.2.21. Panel Definition

5.3.2.23. Part Placement Boundary

5.3.2.24. Part Thermal Outline

5.3.2.25. Pin

5.3.2.29. Via

5.3.2.30. Via Keepin/Keepout

5.3.2.31. Wire Bond 


\subsubsection{Component Placement Keepin/ Keepout}

\section{Description:}

The COMPONENT PLACEMENT KEEPIN/KEEPOUT object is a CLOSED CURVE, which bounds within or excludes the instantiation of all Package Figure Instances.

\section{Requirements/Restrictions:}

The IGES file shall have exactly one COMPONENT PLACEMENT KEEPIN/KEEPOUT per placement layer.

\section{\& Translation Usage Notes:}

\section{General:}

The values for the Generic Property Entity (Type 406, Form 27) shall be as follows:

$$
\text { component_keep_outline }
$$

with a value of either "in," or "out" to state that the component shall be inside or outside the region defined by the closed curve. (NOTE: The CAE system is expected to utilize this object to provide component placement control or rules checking.)

\section{Output:}

Input: see KEEPIN/KEEPOUT (Section 5.3.2.9.). 


\subsubsection{Drawing Definition}

\section{Description:}

The DRAWING DEFINITION object is a Subfigure Definition which represents an engineering drawing of the $L E P$, a $P A N E L$, or some portion of the $L E P$.

\section{Requirements/Restrictions:}

1. The GENERIC DATA PROPERTY which is referenced from the SUBFIGURE DEFINITION object shall specify

$$
\text { date }
$$

2 physical_outline

schematic_number or assembly_number.

2. The NAME field in the SUBFIGURE DEFINITION shall be unique within the scope of the IGES File.

Translation Usage Notes:

General:

Output:

Input:
DRAWING DEFINITION

(LEP CAD Presentation)

0

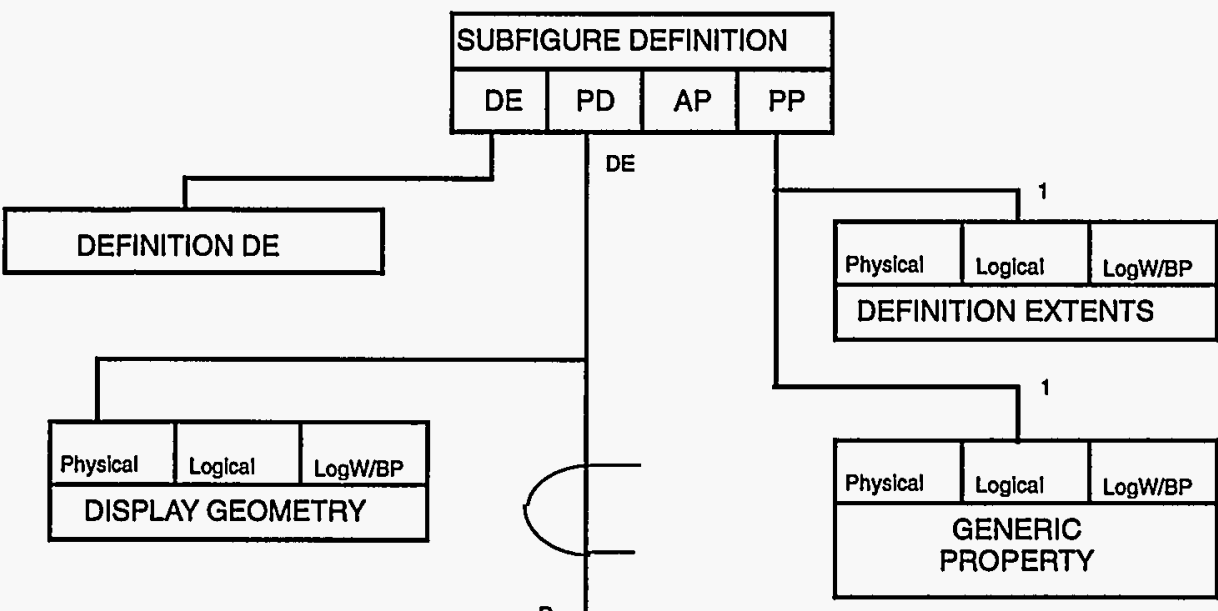

$P$

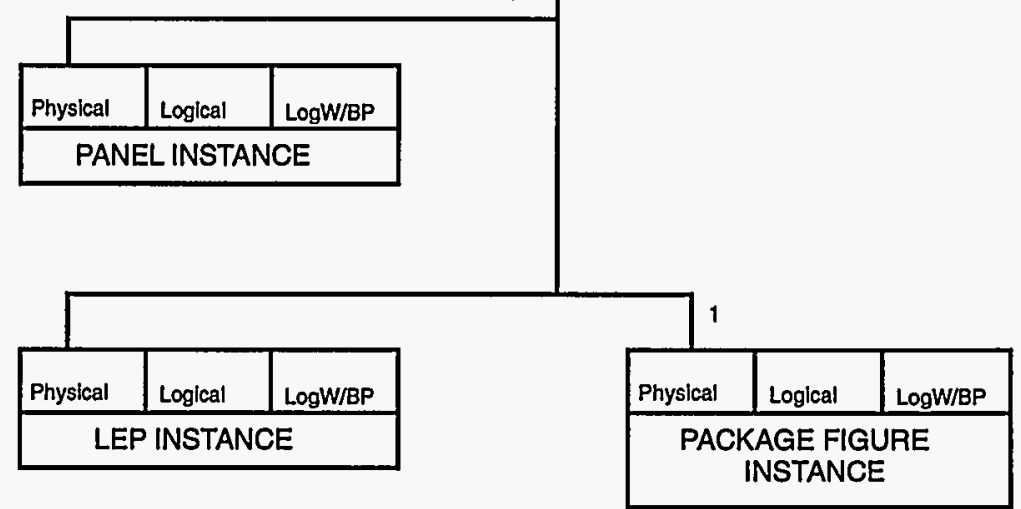




\subsubsection{Drawing Instance}

\section{Description:}

The DRAWING INSTANCE object is an instantiation of a DRAWING DEFINITION.

\section{Requirements/Restrictions:}

1. The GENERIC DATA PROPERTY which is referenced from the SUBFIGURE INSTANCE object shall specify

$$
\text { top_level_assembly_instance. }
$$

\section{Translation Usage Notes:}

今े

General:

Output:

Input:

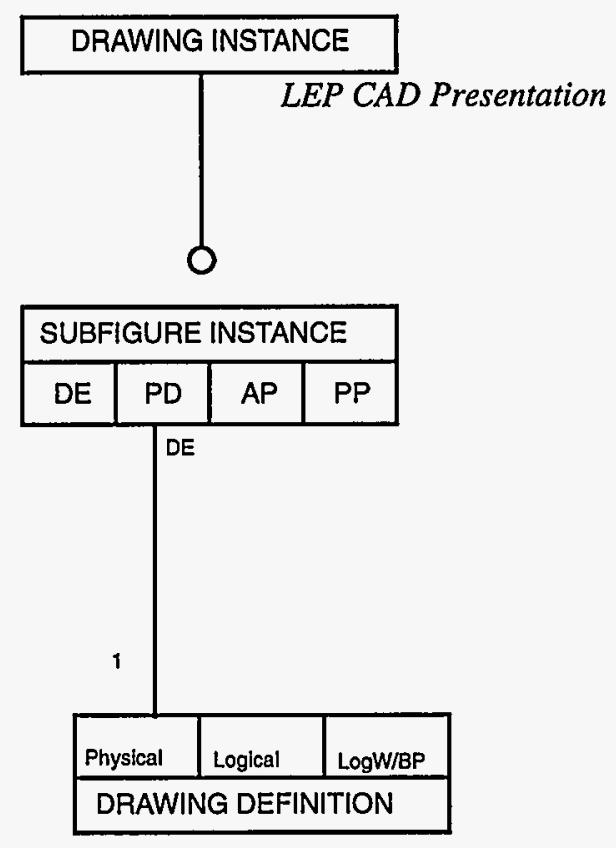




\subsubsection{Generic Part Definition}

\section{Description:}

The GENERIC PART DEFINITION object is a SUBFIGURE DEFINITION, which lists a set of constituent objects for the purpose of treating them like a fixed set or group (e.g., heatsink).

\section{Requirements/Restrictions:}

1. A ROUTING KEEPIN/KEEPOUT or COMPONENT PLACEMENT KEEPIN/KEEPOUT shall not be subordinate to a Generic Part Definition.

2. The GENERIC DATA PROPERTY values referenced

8 from the GENERIC PART DEFINITION are specifed by the PACKAGE SYMBOL DEFINITION.

3. The NAME field in the Subfigure Definition Entity (Type 308) shall be unique (for all Generic Part Definitions) within the scope of the IGES File.

\section{Translation Usage Notes:}

\section{General:}

NOTE: This Generic Part Definition is not applicable to electrical parts; see NETWORK SUBFIGURE DEFINITION.

\section{Output:}

Input:

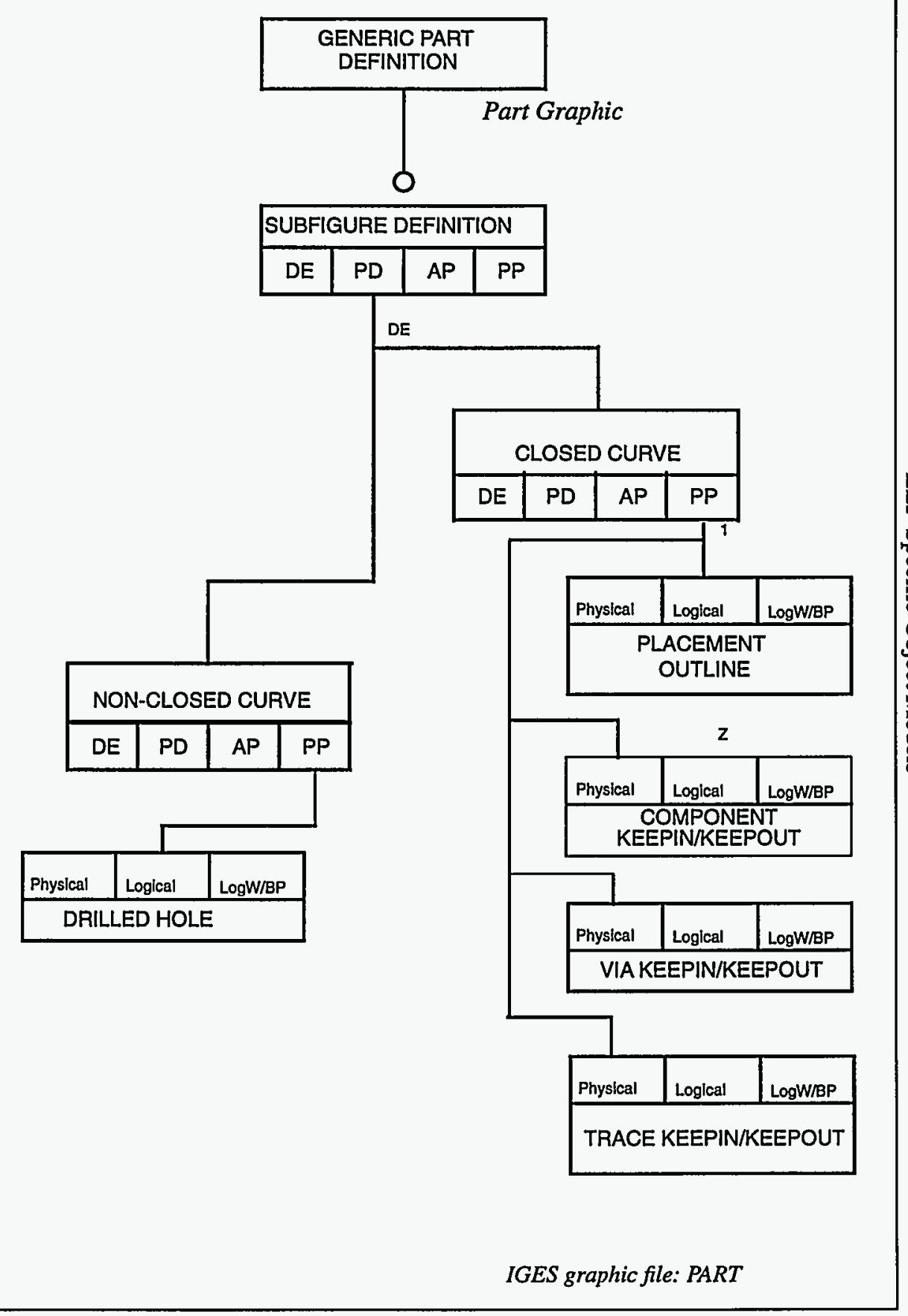




\subsubsection{Generic Part Instance}

\section{Description:}

The GENERIC PART INSTANCE object is an instantiation of a GENERIC PART DEFINITION.

\section{Requirements/Restrictions:}

1. The SEMANTIC ITEM which is referenced from the SUBFIGURE INSTANCE object shall specify.

\section{Translation Usage Notes:}

\section{General: \\ Output:}

क

\section{Input:}

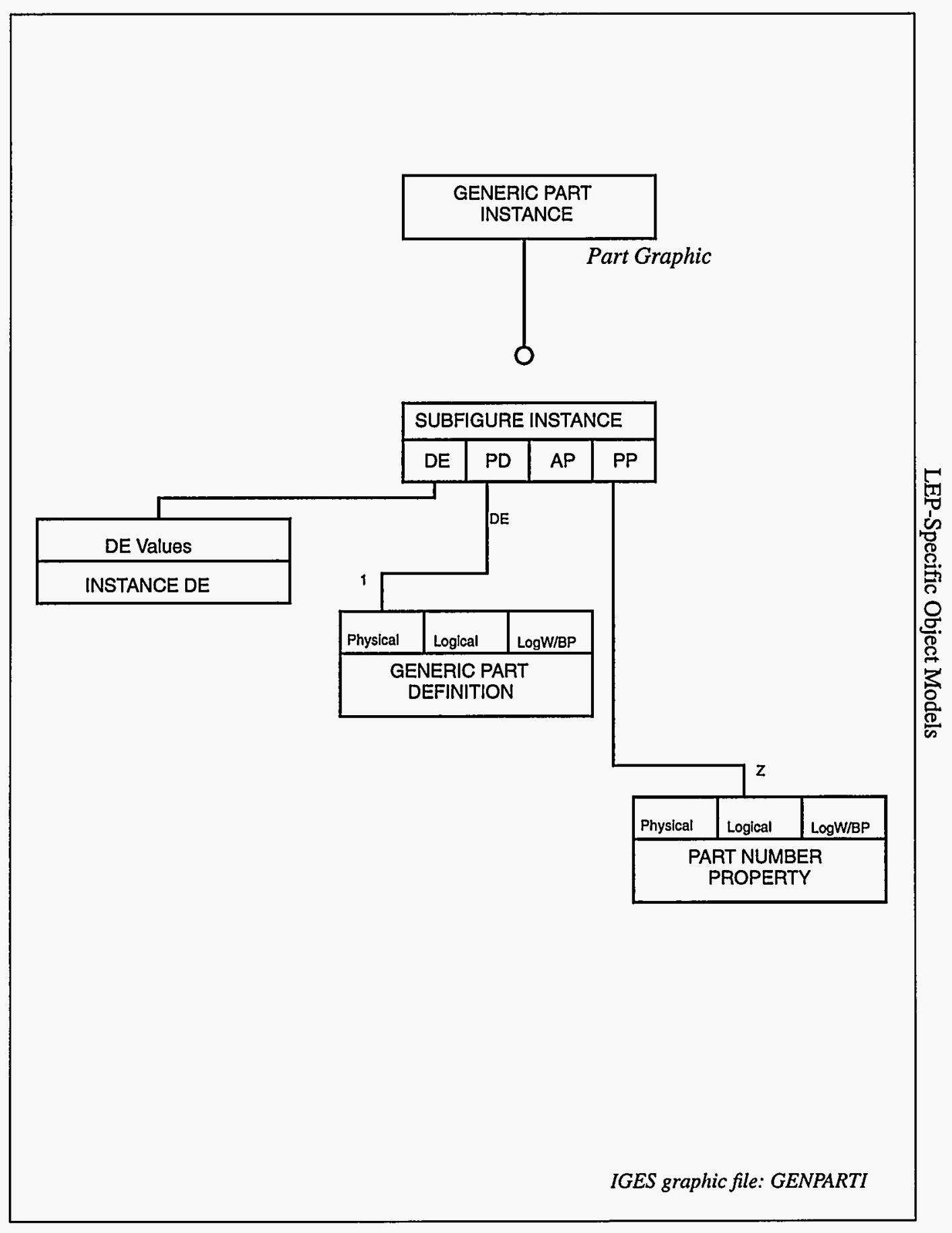




\subsubsection{Hole}

\section{Description:}

The HOLE object documents the location of a hole in one or more layers of the LEP. The hole may be any shape and may be manufactured in a variety of ways (e.g., drilled, punched, milled, or burned).

\section{Requirements/Restrictions:}

1. If the $H O L E$ is subordinate to a definition that is intended to be instantiated on the LEP (e.g., Package Figure Definition), it shall reference a PADSTACK $D E F I N I T I O N$. If the HOLE is subordinate to a LEP

i DEFINITION, it shall reference a PADSTACK INSTANCE.

2. The SEMANTIC ITEM, which is referenced from the POINT or CONNECT POINT object, shall specify physical_outline.

3. The Hole property shall be attached to a Point Entity (Type 116) or Connect Point Entity (Type 132). Either the Point or Connect Point may be independent or dependent (e.g., padstack)

\section{Translation Usage Notes:}

\section{General:}

Output:

Input:

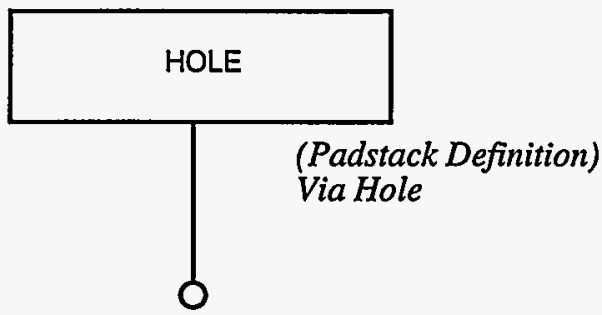

\begin{tabular}{|c|c|c|c|}
\hline \multicolumn{4}{|c|}{ POINT or CONNECT POINT } \\
\hline DE & PD & AP & PP \\
\hline
\end{tabular}




\subsubsection{Join}

\section{Description:}

The JOIN object logically represents any physical conductive material (e.g., conductive filled area, conductive path, or via) that is electrically common within a Net.

\section{Requirements/Restrictions:}

1. A JOIN object shall be part of (pointed to from) a NET.

2. The SEMANTIC ITEM which is referenced from each JOIN object shall be specified as follows:

Generic Property Entity (Type 406, Form 27)

"Name" values

LEP object

Non-Closed Curve

Gen. Prop Name

Closed Curve

Path

Jumper Wire

Area

Via

Jumper Wire

Via

\section{Translation Usage Notes:}

General:

Output:

Input:

Each LEP JOIN object shall include a SEMANTIC

ITEM as defined in requirement 2 above.

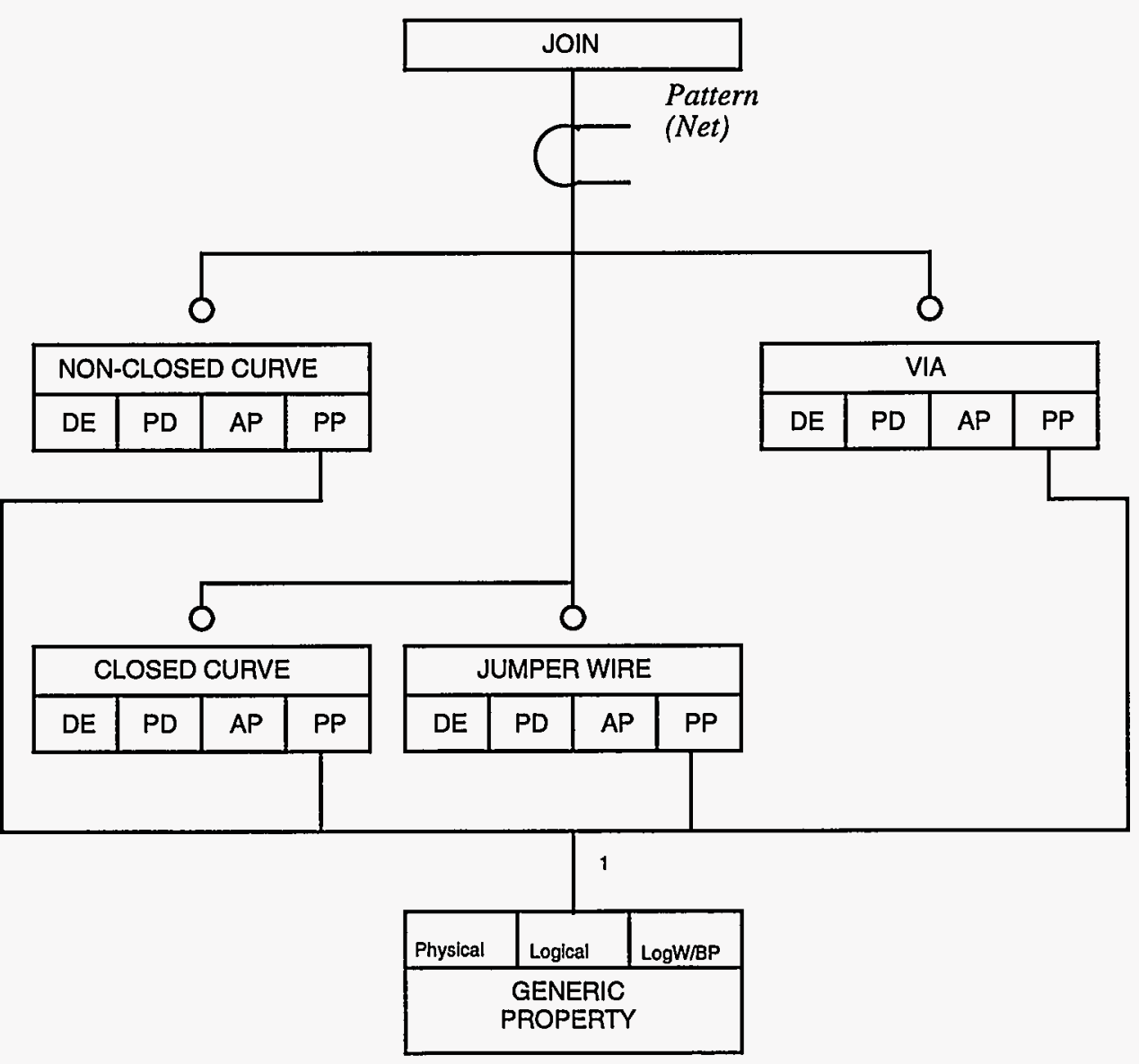

IGES graphic file: JOIN 


\subsubsection{Jumper Wire}

\section{Description:}

The JUMPER WIRE object (often called a "haywire,") is a conductive wire that is used when necessary to electrically connect two or more conductive paths. It is typically used to join two separate substrate nets into one design net.

A WIREBOND is to be encoded as a JUMPER WIRE object.

\section{Requirements/Restrictions:}

9 1. The non-closed curve object shall be tagged with a SEMANTIC ITEM specified as follows: jumper_wire, or wire_bond.

\section{Translation Usage Notes:}

\section{General:}

Output:

\section{Input:}

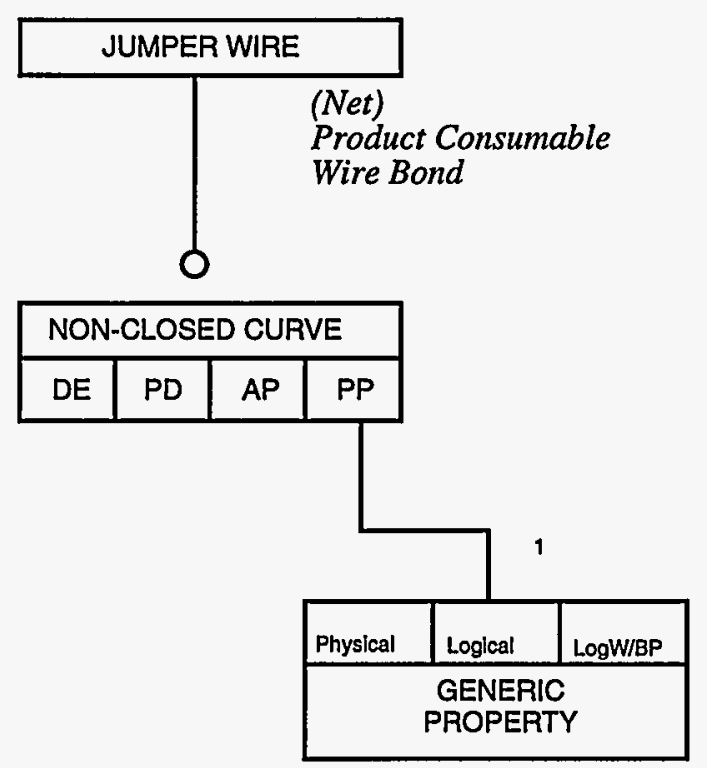




\subsubsection{Keepin/Keepout}

\section{Description:}

A closed-geometry-defined region of an LEP identified as having a design placement restriction.

\section{Requirements/Restrictions:}

\section{Translation Usage Notes:}

\section{General:}

See COMPONENT KEEPIN/KEEPOUT and VIA $K E E P I N / K E E P O U T$ for the appropriate GENERIC DATA PROPERTY which applies.

ळे Output:

Input:

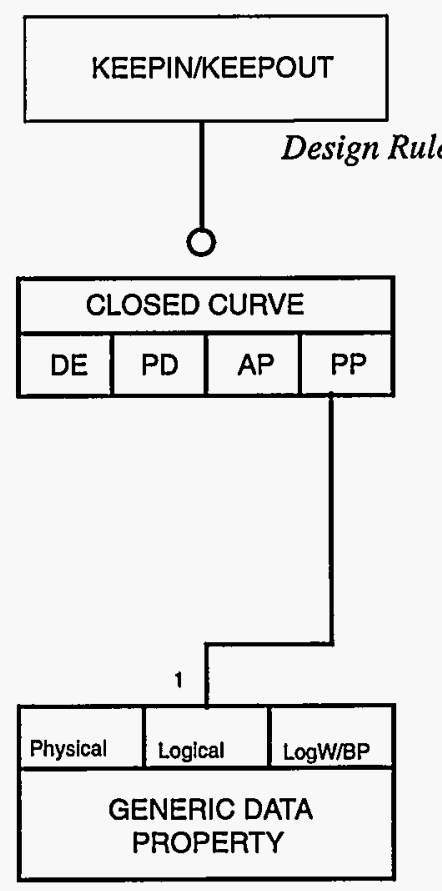




\subsubsection{Land}

\section{Description:}

The $L A N D$ object records and depicts the geometry of a conductive pattern which is used for electrical connection or package PIN attachment.

\section{Requirements/Restrictions:}

\section{Translation Usage Notes:}

\section{General:}

When a $L A N D$ object is subordinate to a PADSTACK I DEFINITION, the IGES file may have distinct land geometry for each possible $L A N D$ subtype. When a $\angle A N D$ object is subordinate to a PADSTACK INSTANCE, the IGES file shall have only one land geometry (anti, regular or thermal) for a particular padstack. The IGES file may have only one LAND object for each LEP functional layer that the particular padstack pierces.
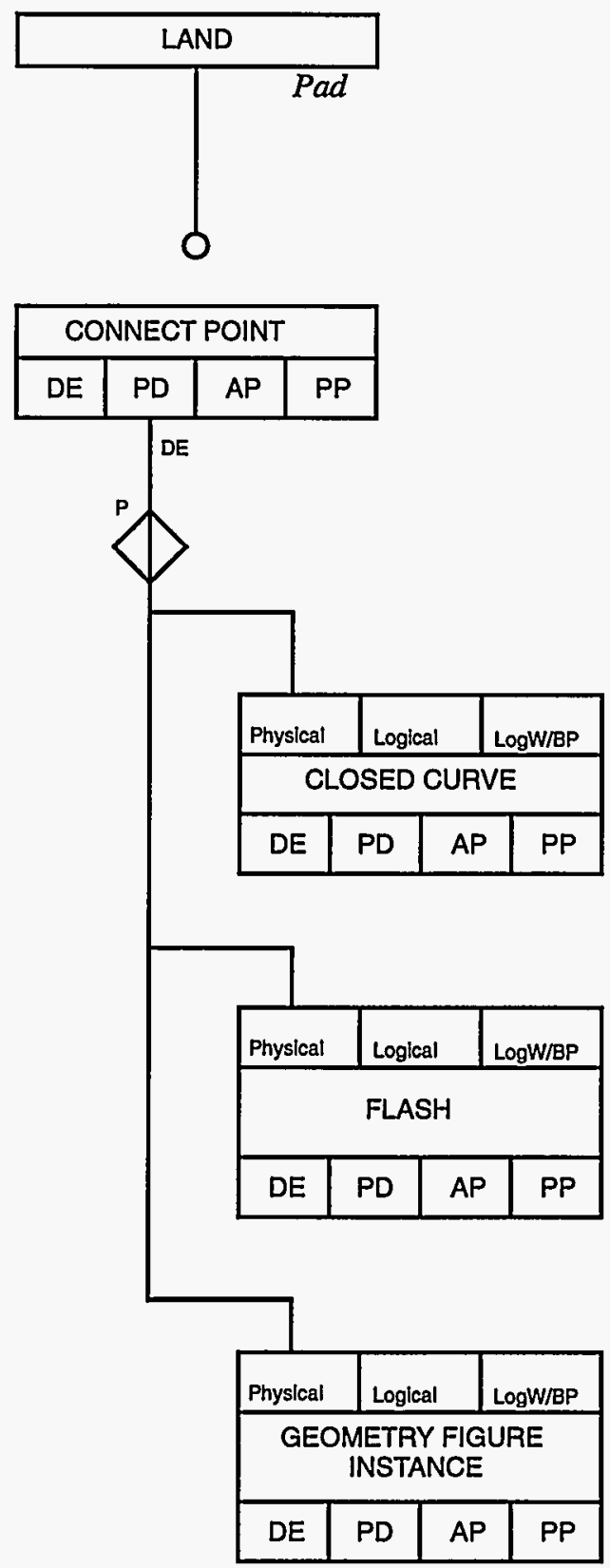


\subsubsection{Layer Outline}

\section{Description:}

The LAYER OUTLINE object depicts the outline (boundary) for a specific physical layer of the LEP (i.e., substrate, power plane, etc.).

\section{Requirements/Restrictions:}

\section{Translation Usage Notes:}

\section{General:}

Exactly one LAYER OUTLINE shall exist for each LEP Physical Layer.

\section{Output:}

Input: see Semantic Item (Section 5.3.5.). 


\subsubsection{LEP Artwork Stackup Property}

\section{Description:}

The LEP ARTWORK STACKUP PROPERTY object maps directly to the IGES PWB Artwork Stackup Property (Type 406, Form 25). Please refer to IGES for additional information.

\section{Requirements/Restrictions:}

Translation Usage Notes:

$\Xi$ General:

Output:

Input: 


\subsubsection{LEP Definition}

\section{Description:}

The LEP DEFINITION OBJECT is a NETWORK SUBFIGURE $D E F I N I T I O N$ object, which relates all of the constituent objects that are required to define the LEP.

\section{Requirements/Restrictions:}

1. The Generic Property Entity (Type 406, Form 27), which is referenced from the NETWORK SUBFIGURE DEFINITION object, shall specify

date

schematic_number, or

$\stackrel{\text { assembly_number. }}{2 \text { 2. The NAME field in the Network Subfigure Definition Entity }}$ (Type 320) shall be unique (for all LEP Definitions) within the scope of the IGES File.

\section{Electrical and LEP Manufacturing Attribute List}

$$
\text { (ALT=5) }
$$

20 Electrostatic Discharge Rating

22 LEP Design Thickness

Translation Usage Notes:

General:

Output:

Input: 

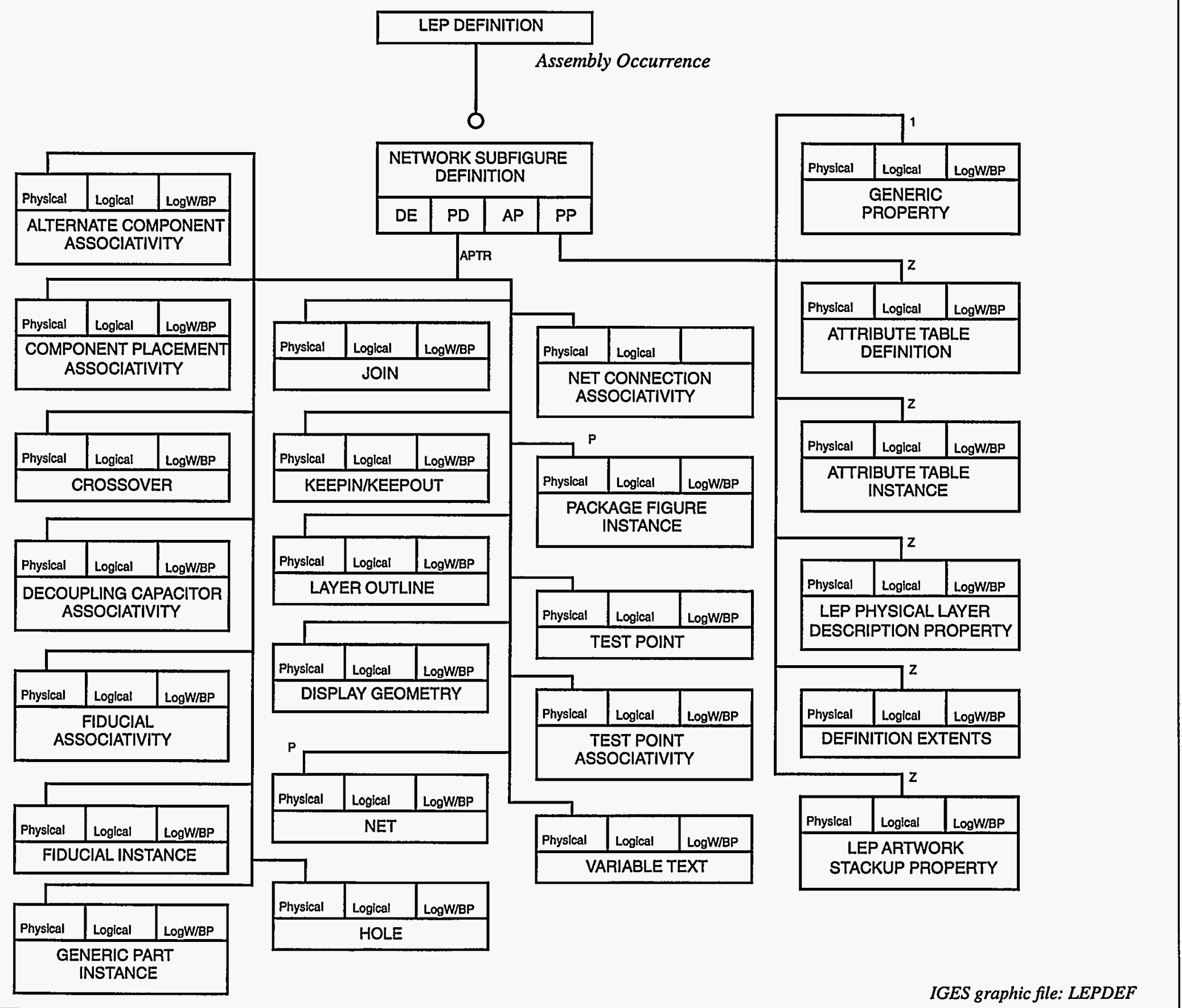


\subsubsection{LEP Instance}

\section{Description:}

The LEP Instance object is an instantiation of an LEP Definition.

\section{Requirements/Restrictions:}

1. The GENERIC DATA PROPERTY, which is referenced from the Network Subfigure Instance object, shall specify

top_level_assembly_instance.

2. The following Attribute Table objects may be referenced from the LEP Instance:

\section{Electrical Attribute List}

6024 Generic Design Rule

\section{Electrical \& LEP Manufacturing Attribute List (ALT=5) \\ 1 Component Physical Orientation \\ 3 Component Physical Thickness \\ 20 Electrostatic Discharge Rating \\ 22 LEP Design Thickness}

\section{Translation Usage Notes:}

\section{General:}

Output:

Input:

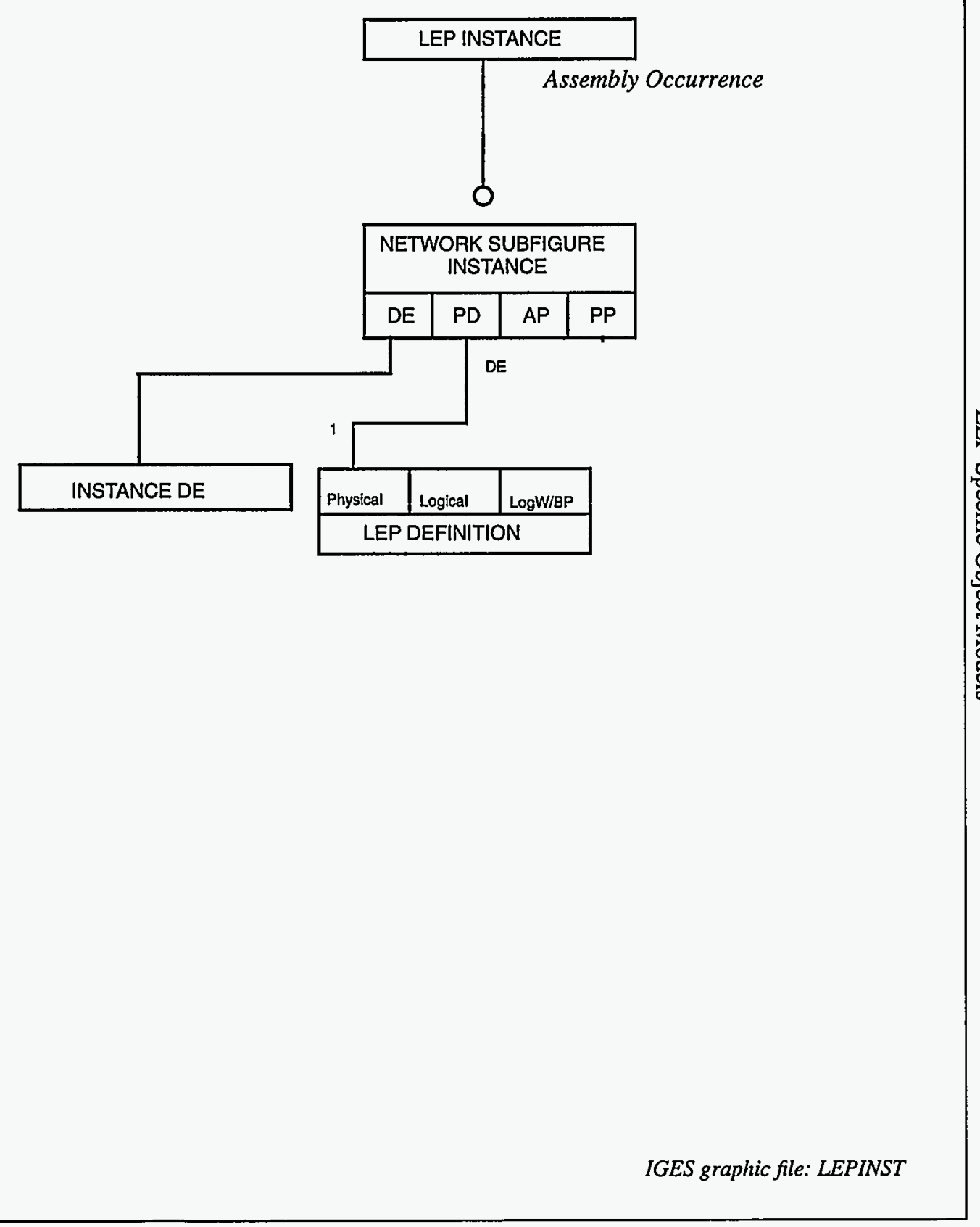




\subsubsection{Level to LEP Layer Map}

\section{Description:}

The LEVEL TO LEP LAYER MAP object maps directly to the IGES Level To LEP Layer Map Property (Type 406, Form 24). Please refer to IGES for additional information.

\section{Requirements/Restrictions:}

1. The Level To LEP Layer Map Property Entity (Type 406, Form 24) is always independent (DE Attribute Subordination equals 0 ) within the IGES file, thus it uे pertains to all other entities in the file.

\section{Translation Usage Notes:}

\section{General:}

\section{Output:}

\section{Input:}

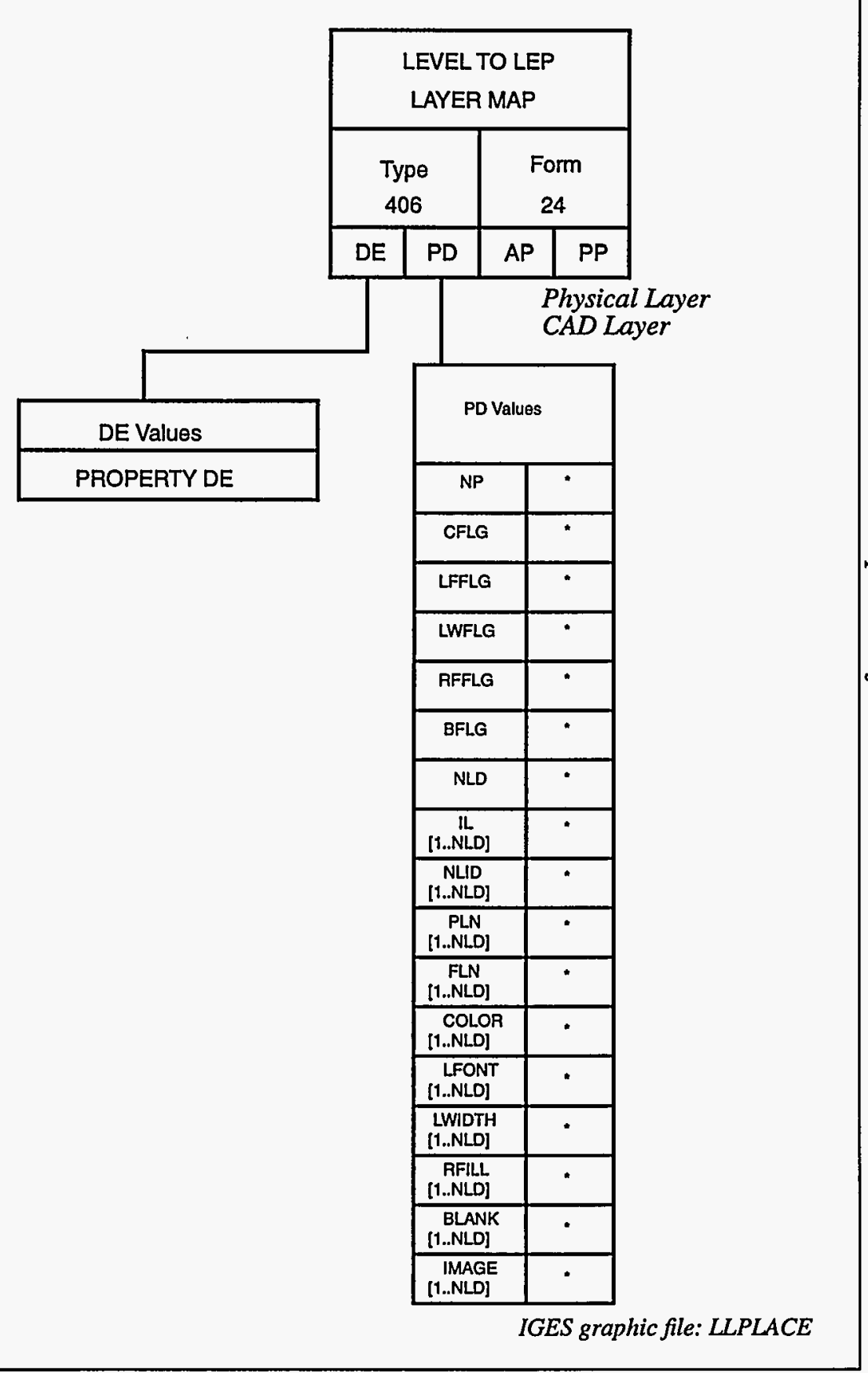




\subsubsection{Net}

\section{Description:}

The NET object associates all of the electrically common PINs and physical JOIN geometry (i.e., conductive filled areas, conductive paths and vias). The NET is intended to represent the LEP design net. If you are concerned with the substrate net you may analyze the structure of the JOINs to determine which Pins are electrically connected at the substrate level (i.e., not through the use of JUMPER WIREs).

\section{Requirements/Restrictions:}

$\sigma 1$. The NAME field in the Flow Associativity Entity (Type 402, Form 18) shall be unique (For all NETs) within the scope of the LEP Definition.

2. Pins may be referenced by a PIN object.

3. All objects that are subordinate to the Flow Associativity Entity (Type 402, Form 18) -with the exception of Pins - shall be physically dependent on the same parent as the Flow Associativity.

4. There shall be 2 or more Pin objects subordinate to the NET.

\section{Translation Usage Notes:}

\section{General:}

The Text Display Template Entity (Type 312)shall be

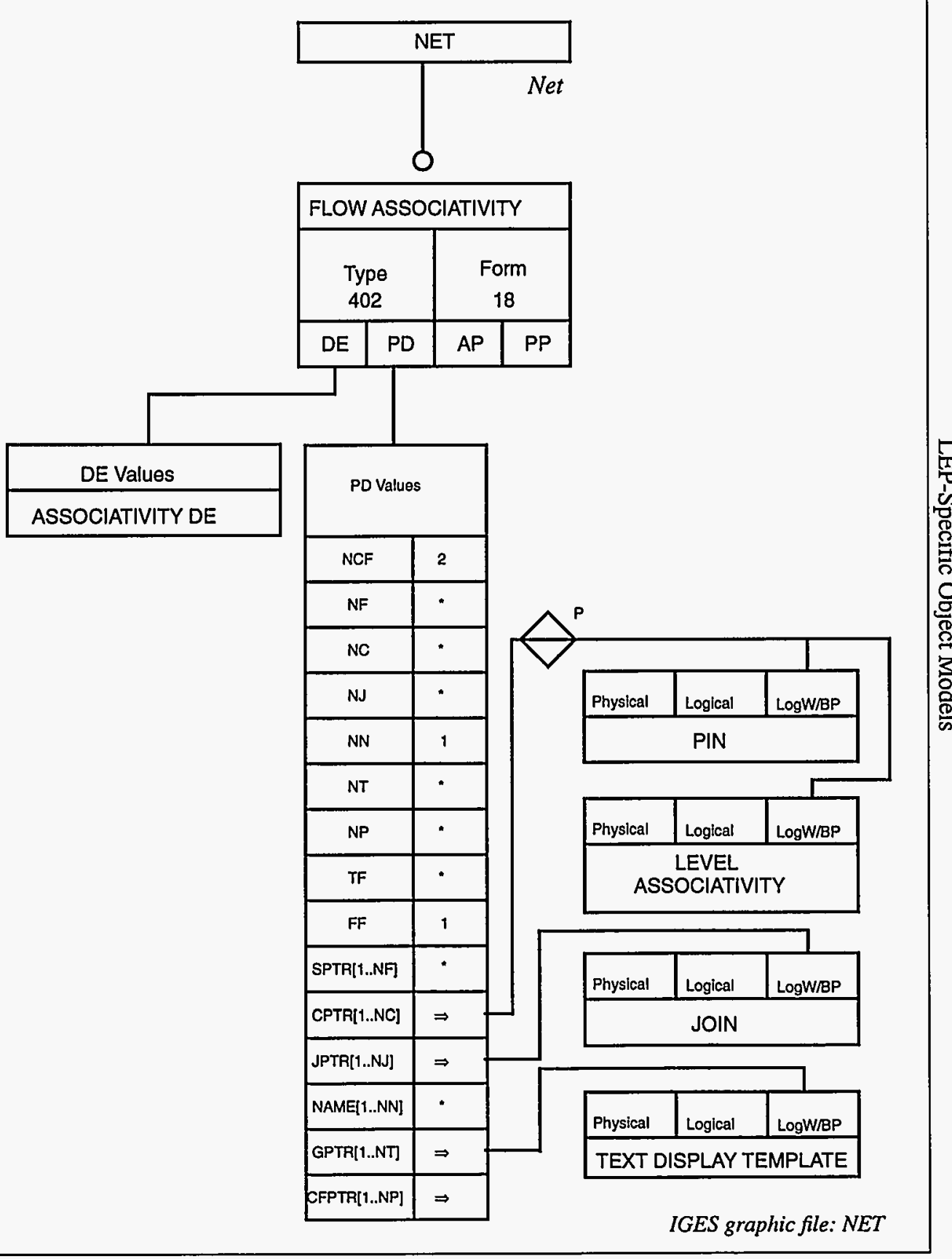


used to display the net name (PD NAME) or identification.

\section{Output:}

Input: 


\subsubsection{Package Symbol Definition}

\section{Description:}

The PACKAGE SYMBOL DEFINITION object is a Subfigure Definition Entity (Type 308) or a Network Subfigure Definition Entity (Type 320), which specifies all of the constituent objects required to define, as a minimum, the "footprint" of a package figure which is assembled to the LEP.

\section{Requirements/Restrictions:}

1. The GENERIC PART DEFINITION object shall be used to represent the definition of a non-electrical component (an attached part).

2. The NETWORK SUBFIGURE DEFINITION object $\infty \quad$ shall be used to represent the definition of an electrical component (an attached electrical part).

3. The GENERIC DATA PROPERTY, which is referenced from the PACKAGE SYMBOL DEFINITIONobject, shall specify

component_std_name

component_version

component_pkg_type

component_tech_type

component_layout_surface

component_insertion_origin

component_placement_feeder_location (*)

component_insertable (*)

component_height

component_max_size

component_outline_overhang $\left(^{*}\right)$.

* When applicable.

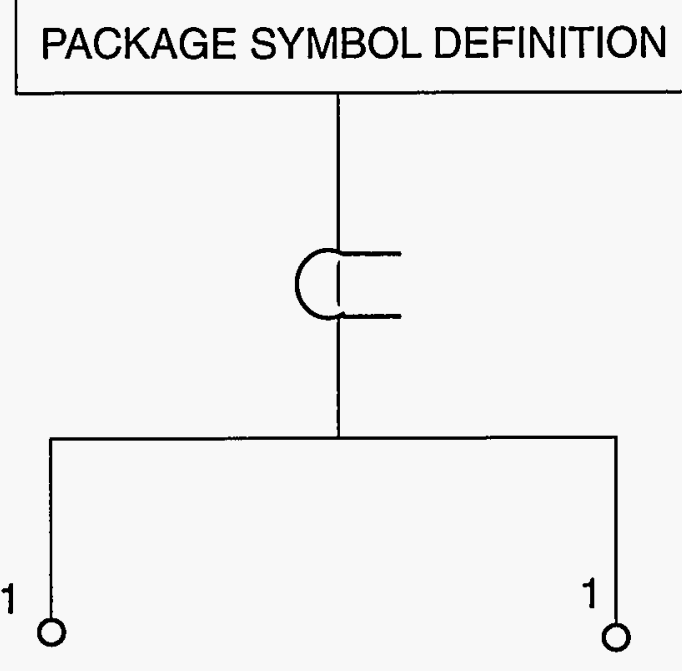

\section{GENERIC PART} DEFINITION 


\section{Translation Usage Notes:}

\section{General:}

The part representation may be a symbolic figure, a minimal outline, a 2 dimension detail, or a 3 dimension

detail as required for the application.

Output:

Input: 


\subsubsection{Package Symbol Instance}

\section{Description:}

The Package Symbol Instance object is a Subfigure Instance Entity (Type 408) or a Network Subfigure Instance Entity (Type 420), which specifies all of the constituent objects required to define, as a minimum, the "footprint" of a package figure which is assembled to the LEP.

\section{Requirements/Restrictions:}

1. The GENERIC PART INSTANCE object shall be used to instance the definition of a non-electrical component (an attached part).

$\stackrel{\circ}{2}$. The NETWORK SUBFIGURE INSTANCE object shall be used to instance the definition of an electrical component (an attached electrical part).

3. The GENERIC DATA PROPERTY, which is referenced from either sub-type of the PACKAGE SYMBOL INSTANCE object, shall specify

abs_voltage_max

component_instance_side

component_preplace $\left(^{*}\right)$

component_via (*)

* When applicable.

Translation Usage Notes:

General:

Output:

Input:

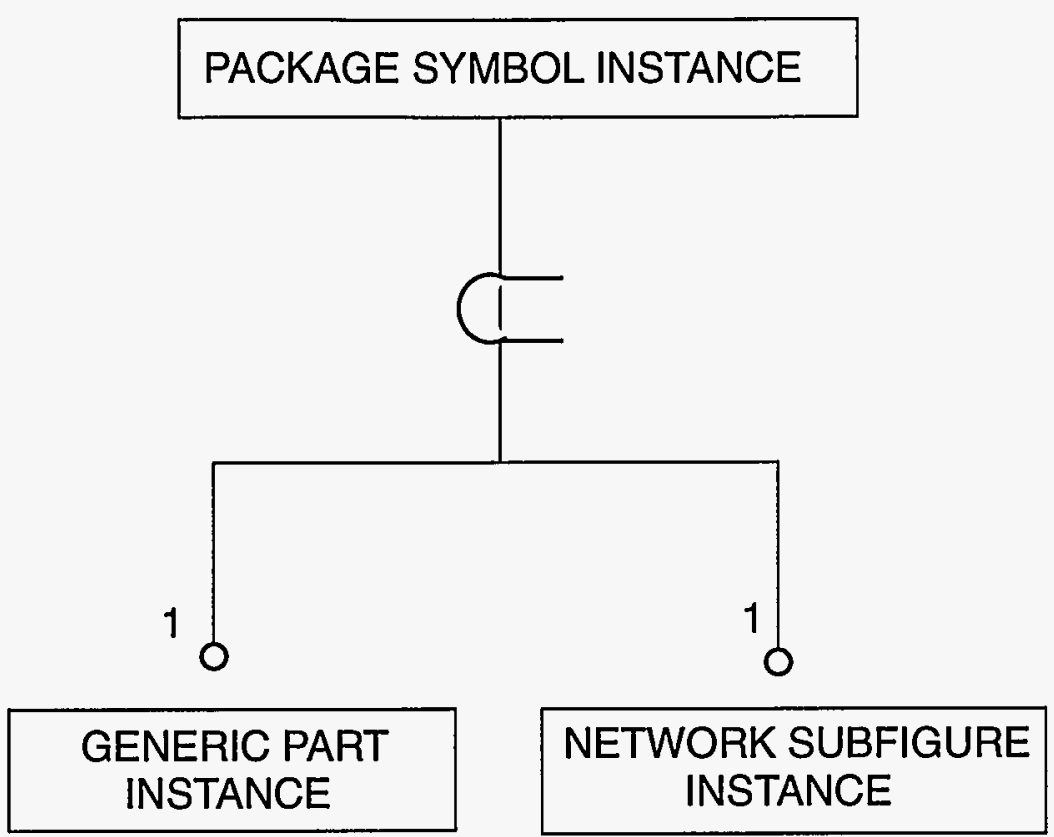




\subsubsection{Padstack Definition}

\section{Description:}

The PADSTACK DEFINITION object is a Subfigure Definition Entity (Type 308), which documents and depicts all of the constituent objects, that are required to list the characteristics of the volume in which a package figure is electrically connected to the LEP.

The characteristics of each layer that is associated with the padstack shall be defined within the PADSTACK DEFINITION.

\section{Requirements/Restrictions:}

$\stackrel{\infty}{\oplus}$

1. The GENERIC DATA PROPERTY, which is referenced from the SUBFIGURE DEFINITION object, shall specify

$$
\begin{aligned}
& \text { physical_padstack_definition } \\
& \text { component_pkg_type (= pad). }
\end{aligned}
$$

2. The NAME field in the Subfigure Definition shall be unique (for all Padstack Definitions) within the scope of the IGES File.

\section{Translation Usage Notes:}

\section{General:}

The DE Level attribute of the HOLE shall be used to specify which levels (physical layers) the padstack pierces. In order to affect more than one level, a LEVEL DEFINITION object shall be used.

\section{Output:}

Input:

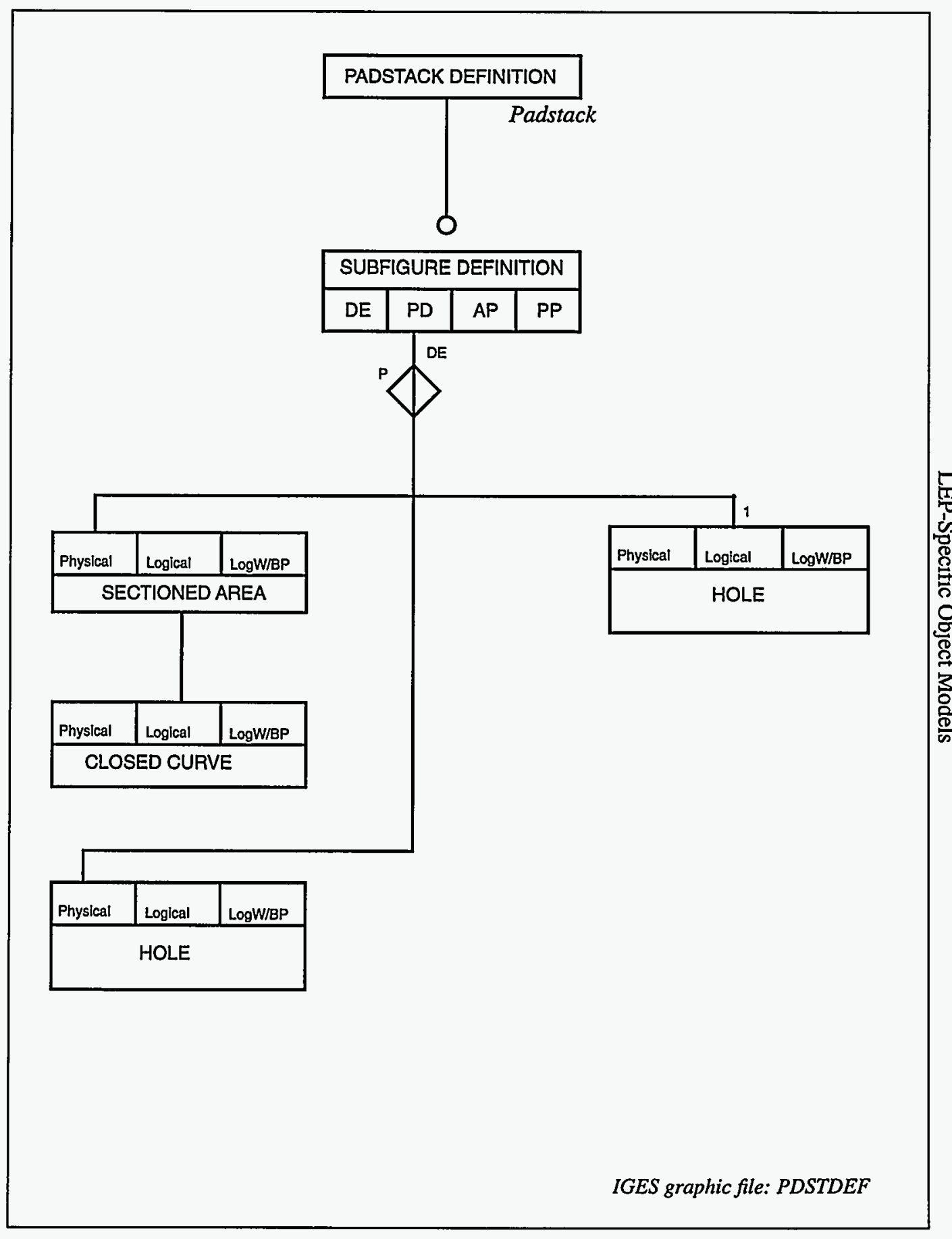




\subsubsection{Padstack Instance}

\section{Description:}

The PADSTACK INSTANCE object is an instantiation of a PADSTACK DEFINITION.

\section{Requirements/Restrictions:}

\section{Translation Usage Notes:}

\section{General:}

The DE Level attribute is used to indicate which levels (physical layers) the padstack affects. In order to affect $\infty$ more than one level, a LEVEL DEFINITION object shall be used.

\section{Output:}

Input:

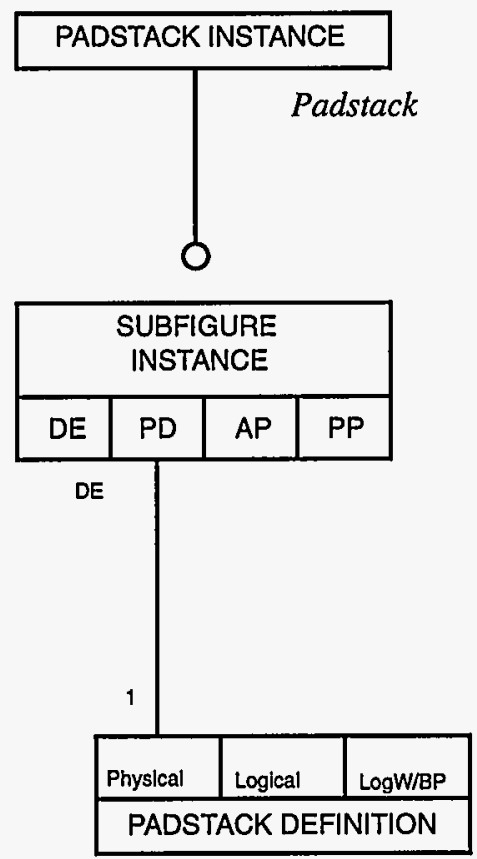




\subsubsection{Panel Definition}

\section{Description:}

The PANEL DEFINITION object is a Subfigure Definition Entity (Type 308), which relates all of the constituent objects that are required to depict a manufacturing panel.

\section{Requirements/Restrictions:}

1. The GENERIC DATA PROPERTY, which is referenced from the GENERIC PART DEFINITION object, shall specify

$$
\text { panel. }
$$

$\stackrel{\infty}{\omega}$ 2. The NAME field in the SUBFIGURE DEFINITION shall be unique (for all PANEL DEFINITIONs) within the scope of the IGES File.

\section{Translation Usage Notes:}

\section{General:}

The Display Geometry is included only as needed to describe the Panel.

\section{Output:}

\section{Input:}

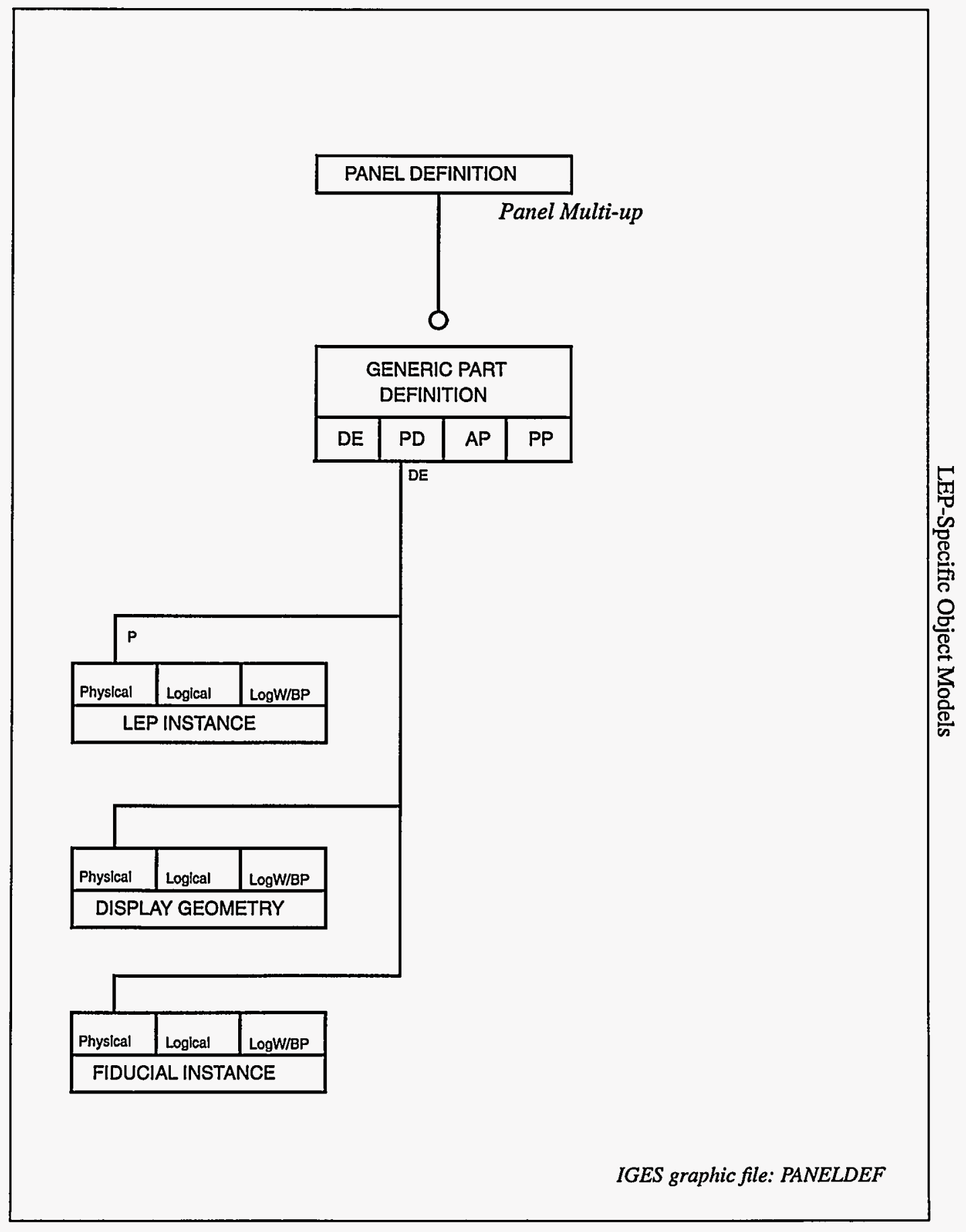




\subsubsection{Panel Instance}

\section{Description:}

The PANEL INSTANCE object is an instantiation of a PANEL DEFINITION.

\section{Requirements/Restrictions:}

1. The PANEL INSTANCE may be implemented using a Subfigure Instance Entity (Type 408).

\section{Translation Usage Notes:}

\section{General:}

$\stackrel{\infty}{+}$

\section{Output:}

\section{Input:}




\subsubsection{Part Placement Boundary}

\section{Description:}

The PART PLACEMENT BOUNDARY object is a closed curve, which shows the area on the LEP that a Package Figure Definition encompasses. The Part Placement Boundary encompasses a volume formed by an $\mathrm{XY}$ area plus a lower and upper height for the area.

\section{Requirements/Restrictions:}

1. The CLOSED CURVE shall only be an Arc, a Simple Planar Curve Entity (Type 106, Form 63), or a Subfigure Definition Entity (Type 308) which is coplanar, closed, and non-crossing.

i 2. The GENERIC DATA PROPERTY, which is referenced from the GENERIC PART DEFINITION object, shall specify

$$
\text { component_max_size. }
$$

\section{Translation Usage Notes:}

This object may exist on any level.

General:

\section{Output:}

\section{Input:}

LEP 'Part Placement_Outline' Property is an IGES Generic Property Entity (Type 406, Form 27).

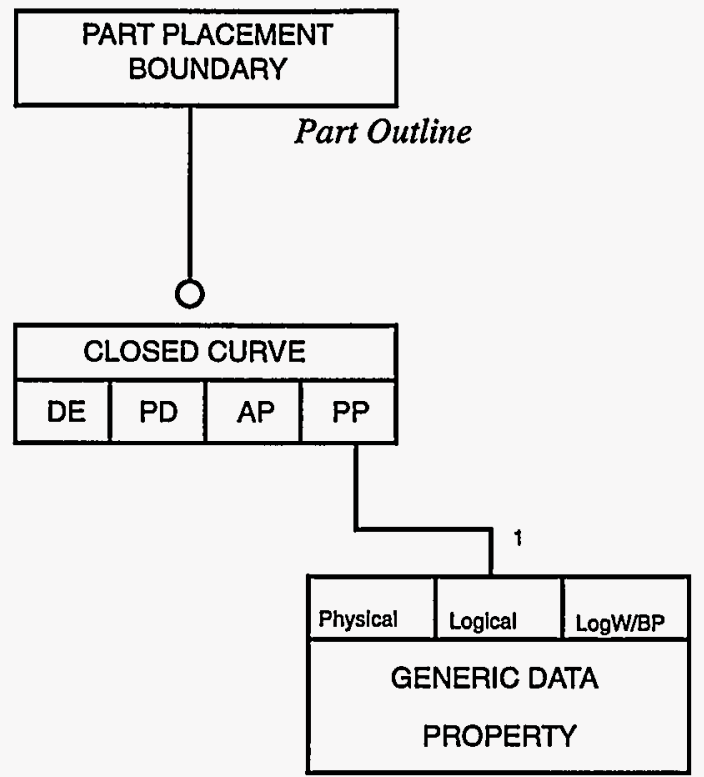




\subsubsection{Part Thermal Outline}

\section{Description:}

The PART THERMAL OUTLINE object is a CLOSED CURVE, which represents the area of the PACKAGE SYMBOL INSTANCE that has associated thermal characteristics.

\section{Requirements/Restrictions:}

Translation Usage Notes:

\section{General:}

$\approx$ The value for the Generic Property Entity (Type 406, Form 27) shall be:

$$
\text { thermal_outline }
$$

to state that the component has a thermal boundary defined at the closed curve.

Output:

Input:

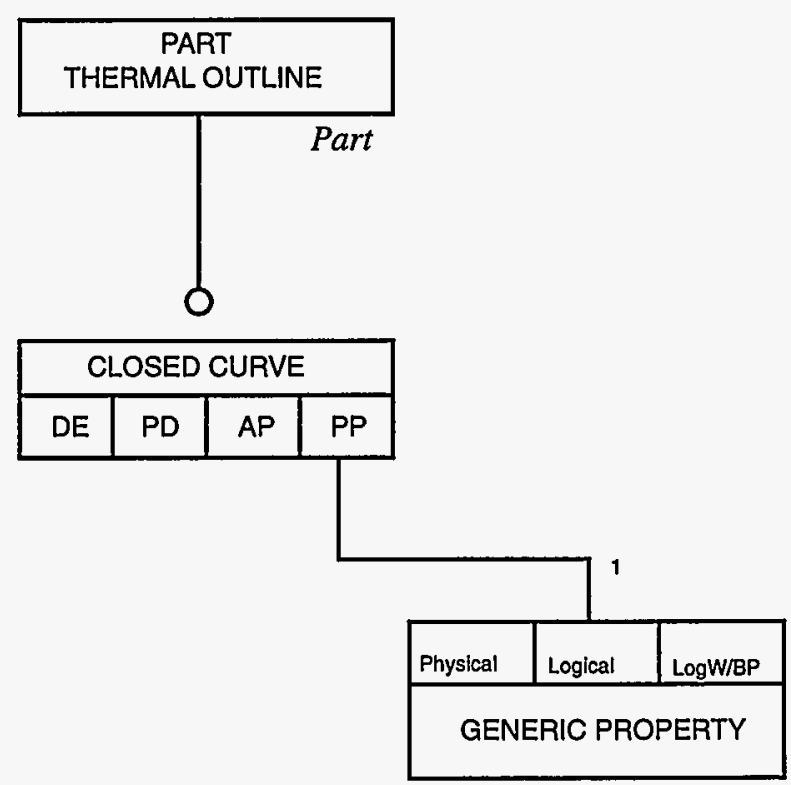




\subsubsection{Pin}

\section{Description:}

The PIN object documents the location, and functional characteristics of the electrical connection of a package pin to the LEP.

\section{Requirements/Restrictions:}

1. There is no subordination associated with the Subfigure Instance (PSFI) pointer. It is an informational reference only.

2. The Connection Point Identifier (CID) field in the Connect Point Entity (Type 132) shall be unique

$\$$ within the scope of the Package Figure Definition or Package Figure Instance.

3. The Connect Point Identifier (CPID) field in the Connect Point shall be unique within the scope of the Package Figure Definition or Package Figure Instance.

4. The following Attribute Table objects may be referenced from the Pin.

\section{Electrical Attribute List (ALT=2)}

\section{Pin Size \\ 6024 Generic Design Rule}

Electrical and LEP Manufacturing Attribute List

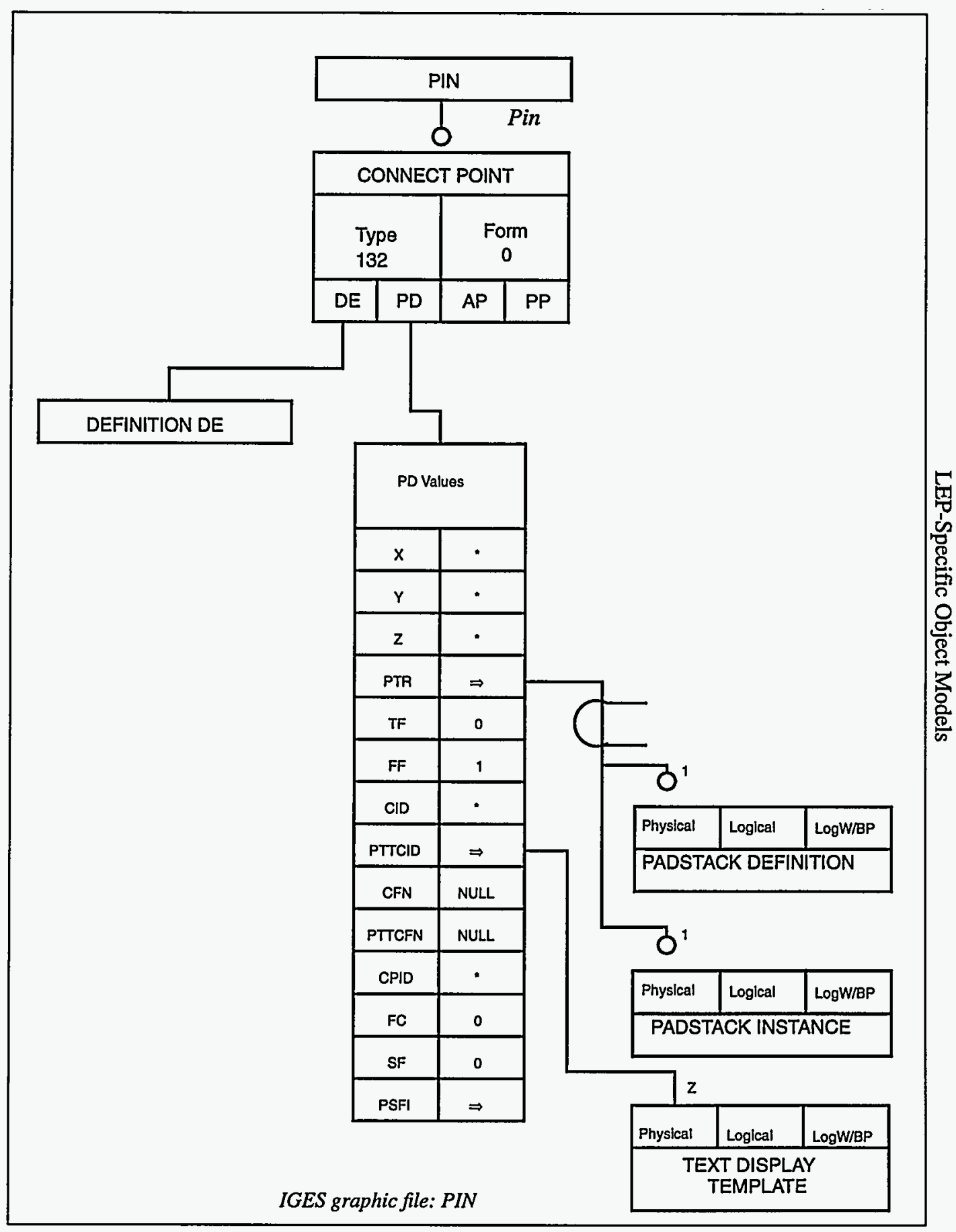




\section{$(\mathrm{ALT}=5)$}

4 Component Placement Lead Forming

\section{Translation Usage Notes:}

\section{General:}

The value for the Generic Property Entity (Type 406, Form 27)

for the PIN designated as "pin 1" shall be:

$$
\text { pin1 }
$$

\section{Output:}

Input: 


\subsubsection{Routing Keepin/Keepout}

\section{Description:}

The ROUTING KEEPIN object is a CLOSED CURVE, which represents an outline which totally encloses all routing objects (e.g., conductive filled areas, conductive paths, and vias).

\section{Requirements/Restrictions:}

Translation Usage Notes:

General:

\&

Output:

Input:

see SEMANTIC ITEM (Section 5.3.5.). 


\subsubsection{Test Point}

\section{Description:}

see SEMANTIC ITEM (Section 5.3.5.).

The TEST POINT object indicates a location which is designated for probing during a specific electrical test procedure (e.g., in-circuit, bare board, functional).

\section{Requirements/Restrictions:}

Translation Usage Notes:

General:

¿ Output:

Input: 


\subsubsection{Trace Keepout}

\section{Description:}

see SEMANTIC ITEM (Section 5.3.5.).

The TRACE KEEPOUT object is a closed curve, which represents an outline in which there shall be no traces

(e.g., conductive areas or conductive paths).

\section{Requirements/Restrictions:}

Translation Usage Notes:

General:

O Output:

Input: 


\subsubsection{Via}

\section{Description:}

The VIA object represents a special type of Join. It is used to electrically connect conductive material between the physical layers of the LEP.

\section{Requirements/Restrictions:}

1. The GENERIC DATA PROPERTY, if referenced from the $P I N$ object, shall specify

$$
\text { component_via. }
$$

2. If the Via is subordinate to a definition that is intended to be instantiated on the LEP (i.e., Package

i Figure Definition), it shall reference a Padstack Definition. If the Via is subordinate to a LEP Definition, it shall reference a Padstack Instance.

\section{Translation Usage Notes:}

\section{General:}

\section{Output:}

\section{Input:}




\subsubsection{Via Keepin/Keepout}

\section{Description:}

The VIA KEEPIN/KEEPOUT object is a CLOSED $C U R V E$, which represents an outline in which there shall be no Vias.

\section{Requirements/Restrictions:}

\section{Translation Usage Notes:}

\section{General:}

The values for the Generic Property Entity (Type 406, i Form 27) shall be as follows: via_keep_outline

Output:

Input: 


\subsubsection{Wire Bond}

\section{Description:}

The WIRE BOND object is a special type of JOIN. It is used to electrically connect a pre-packaged IC or HMA to the surface of an MCM. A Wire Bond is typically soldered, thermally bonded, or compression bonded to a special type of Padstack (wire bond pad).

\section{Requirements/Restrictions:}

1. The Generic Property Entity (Type 406, Form 27), which is referenced from the Non-Closed Curve object, shall specify

$$
\begin{aligned}
& \text { wire_wrap } \\
& \text { gauge } \\
& \text { wire_color } \\
& \text { twisted_pair } \\
& \text { wrap_class (optional). }
\end{aligned}
$$

\section{Translation Usage Notes:}

\section{General:}

\section{Output:}

Input: 


\subsubsection{Display Geometry Object}

\section{Models}

These objects are used to depict shapes of physical objects in the layered electrical product and are also common to files describing mechanical products.

\subsubsection{Arc}

\section{Description:}

The $A R C$ object represents a non-closed circular arc.

\section{Requirements/Restrictions:}

识 1. The start and end point of the IGES Circular Arc Entity (Type 100) shall not be coincident (with respect to the Global Section Minimum User-Intended Resolution).

2. The radius shall be greater than or equal to the Minimum User-Intended Resolution.

\section{Translation Usage Notes:}

\section{General:}

Output:

Input:

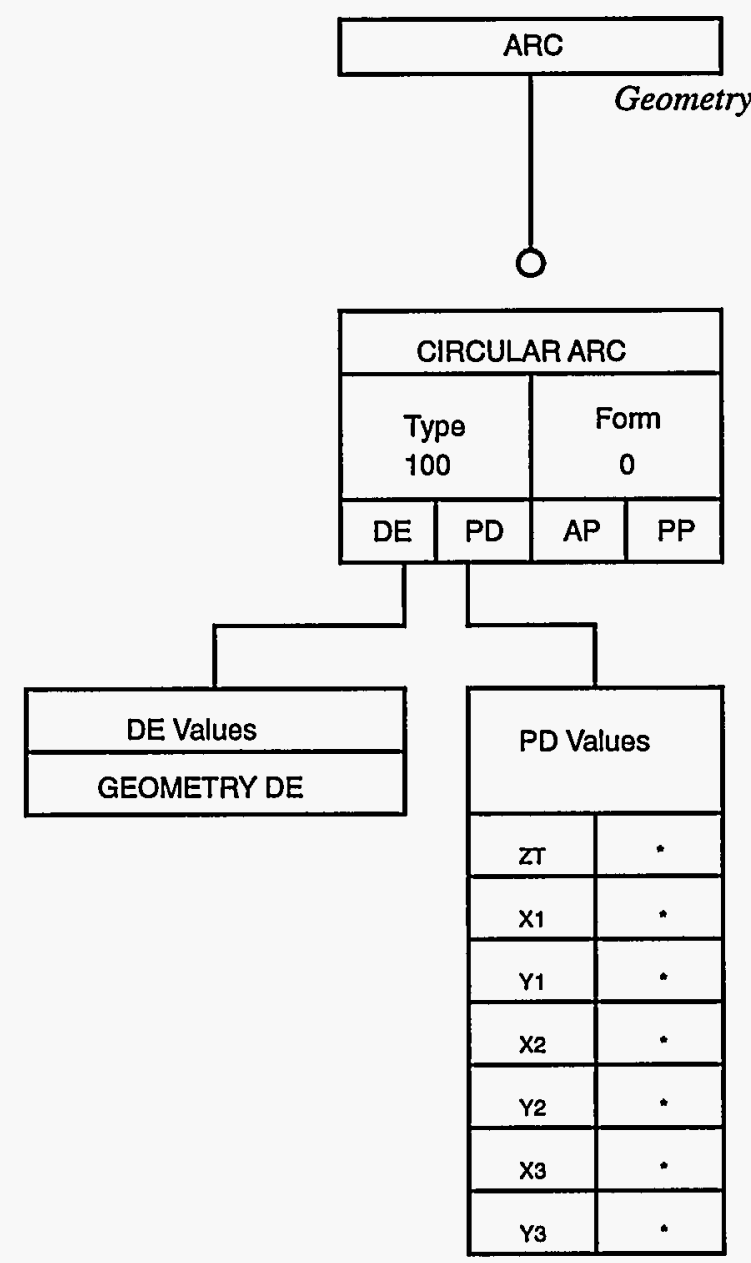

IGES graphic file: ARC 


\subsubsection{Circle}

\section{Description:}

The CIRCLE object represents a closed circular arc.

\section{Requirements/Restrictions:}

1. The start and end point of the IGES Circular Arc Entity (Type 100) shall be coincident (with respect to the Global Section Minimum User-Intended Resolution).

2. The radius shall be greater than or equal to the Minimum User-Intended Resolution.

\section{ฉ̊ Translation Usage Notes:}

\section{General:}

\section{Output:}

\section{Input:}

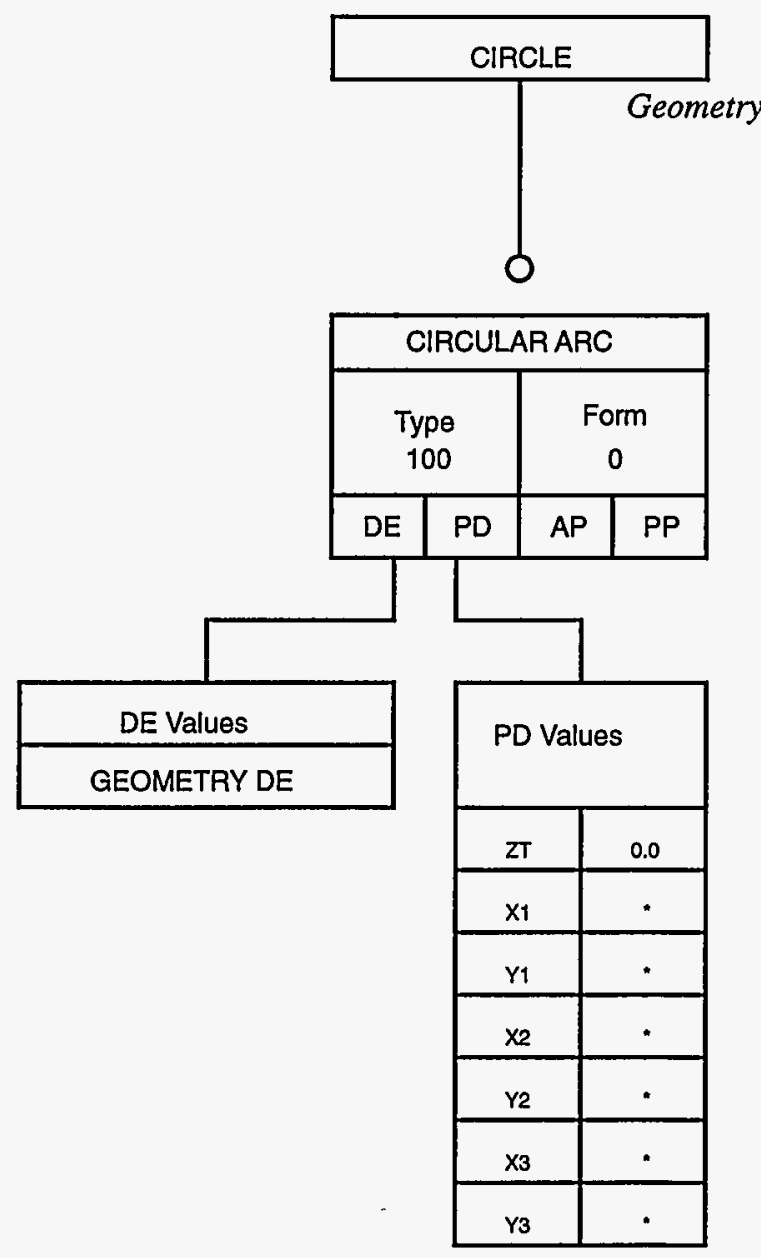

IGES graphic file: CIRCLE 


\subsubsection{Closed Curve}

\section{Description:}

The CLOSED CURVE object is any one of the predefined curves which is capable of defining a closed curve.

\section{Requirements/Restrictions:}

1. A CLOSED CURVE shall not self intersect or become tangent with itself at any point other than the start point which shall be coincident with the end point.

\section{Translation Usage Notes:}

q General:

Output:

Input: 


\subsubsection{Geometry Figure Definition}

\section{Description:}

The GEOMETRY FIGURE DEFINITION object is a Subfigure Definition Entity (Type 308), which contains only graphic display geometry objects.

\section{Requirements/Restrictions:}

1. The LEP Object Type/Sub-Type property, which is referenced from the Subfigure Definition object, shall specify (otype $=$ Geometry_Figure, stype $=*$ ).

2. The NAME field in the Subfigure Definition shall be unique (for all Geometry Figure Definitions) within

$® \quad$ the scope of the IGES File.

\section{Translation Usage Notes:}

\section{General:}

Output:
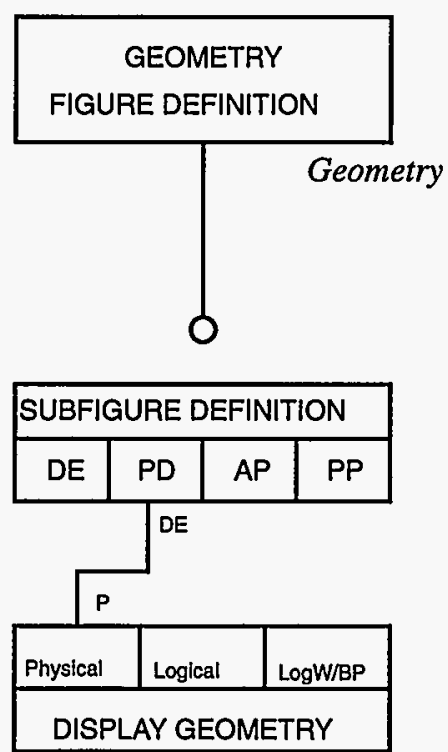

Input: 


\subsubsection{Geometry Figure Instance}

\section{Description:}

The GEOMETRY FIGURE INSTANCE Object is either a Simple Geometry Figure Instance or a Modified Geometry Figure Instance.

\section{Requirements/Restrictions:}

1. The LEP Object Type/Sub-Type property, which is referenced from the Subfigure Instance object, shall specify (otype $=$ Geometry_Figure, stype $=*$ ).

Translation Usage Notes:

\&

\section{General:}

Output:

Input:

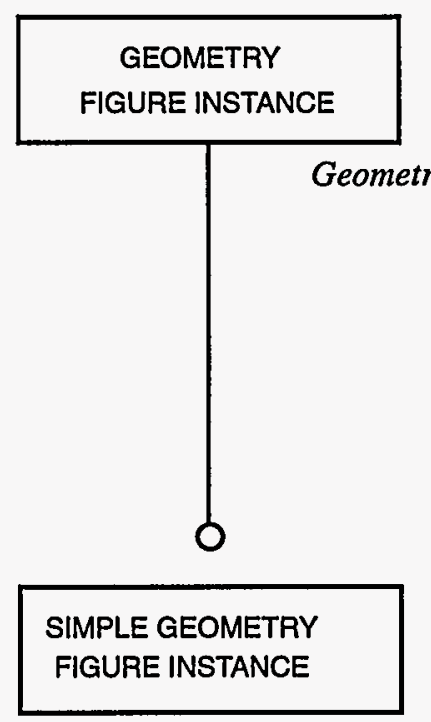




\subsubsection{Line}

\section{Description:}

The LINE object represents a line segment.

\section{Requirements/Restrictions:}

1. The start and end point of the IGES Line Entity (Type 110) must not be coincident (with respect to the Global Section Minimum User-Intended Resolution).

\section{Translation Usage Notes:}

\section{General:}

$\overrightarrow{8}_{\text {Output: }}$

Input:

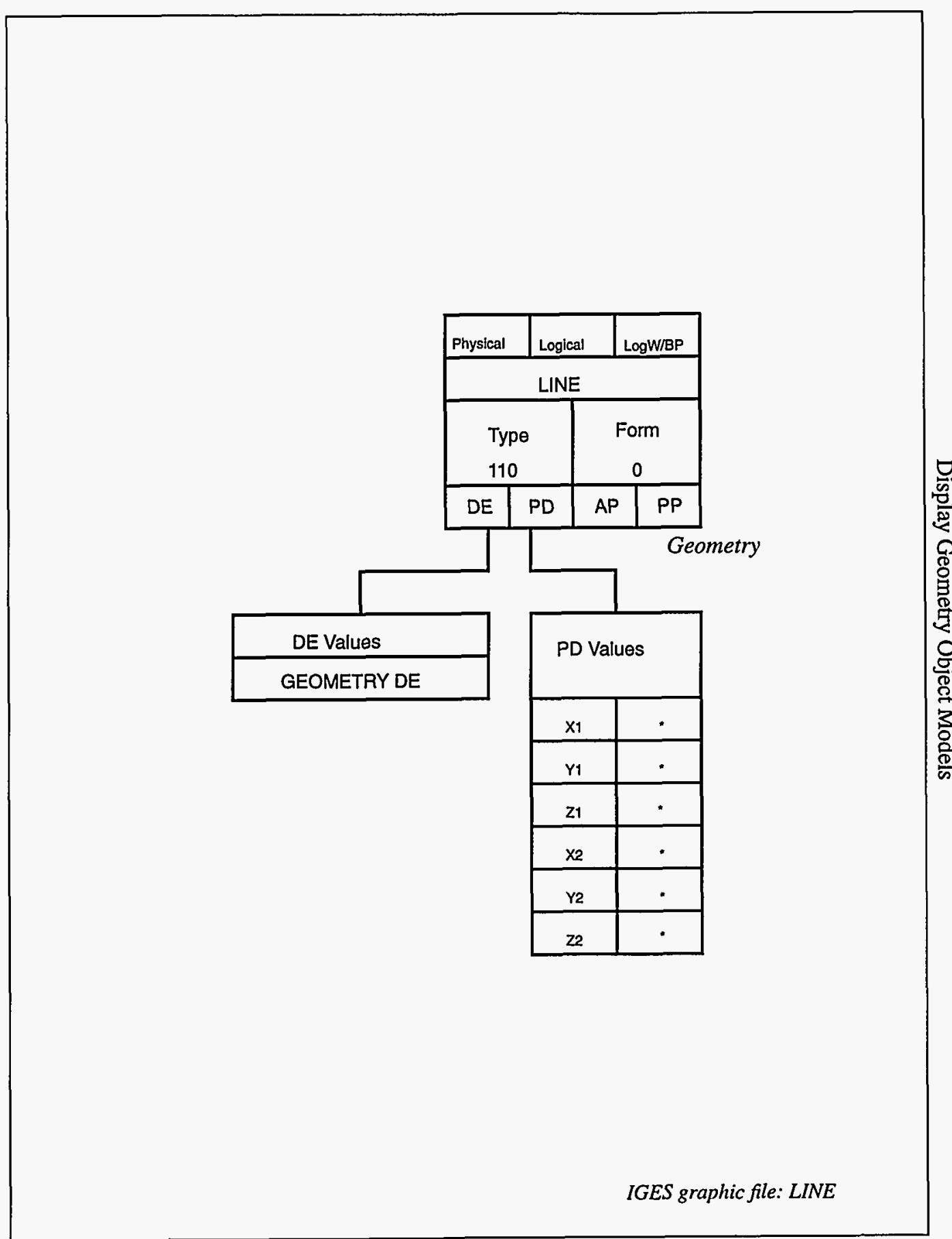




\subsubsection{Multi-Line}

\section{Description:}

The MULTI-LINE object represents more than two points connected by a series of line segments. MultiLines are not closed curves.

\section{Requirements/Restrictions:}

1. The MULTI-LINE may or may not be closed.

2. The MULTI-LINE shall contain more than two points.

\section{Translation Usage Notes:}

๖ General:

\section{Output:}

Input:

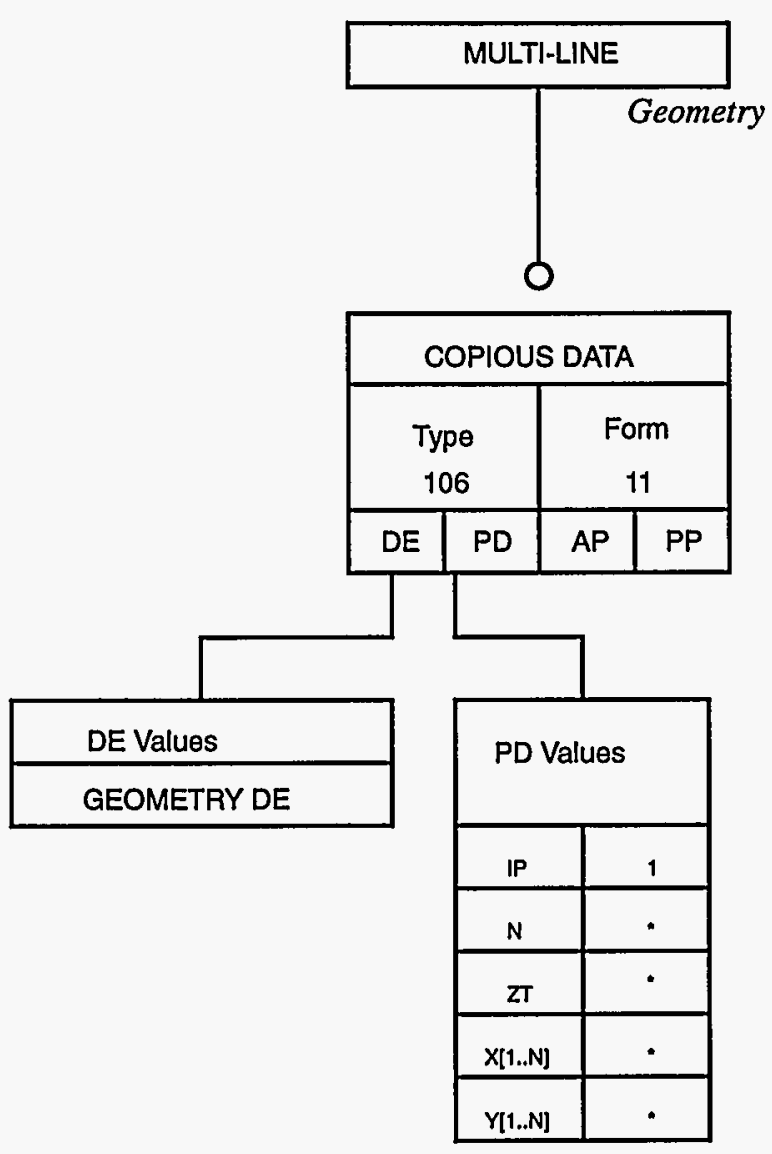




\subsubsection{Polygon}

\section{Description:}

The POLYGON object is a closed multi-line that does not self intersect nor become tangent with itself at any point, other than the start and end point. Because the polygon is a CLOSED CURVE, the start point and end point are coincident.

\section{Requirements/Restrictions:}

\section{Translation Usage Notes:}

$\sigma^{\circ}$ General:

Output:

Input: 


\subsubsection{Point}

\section{Description:}

The POINT object represents a 2-D geometric construction point.

\section{Requirements/Restrictions:}

\section{Translation Usage Notes:}

\section{General:}

Output:

'̊̊ Input:

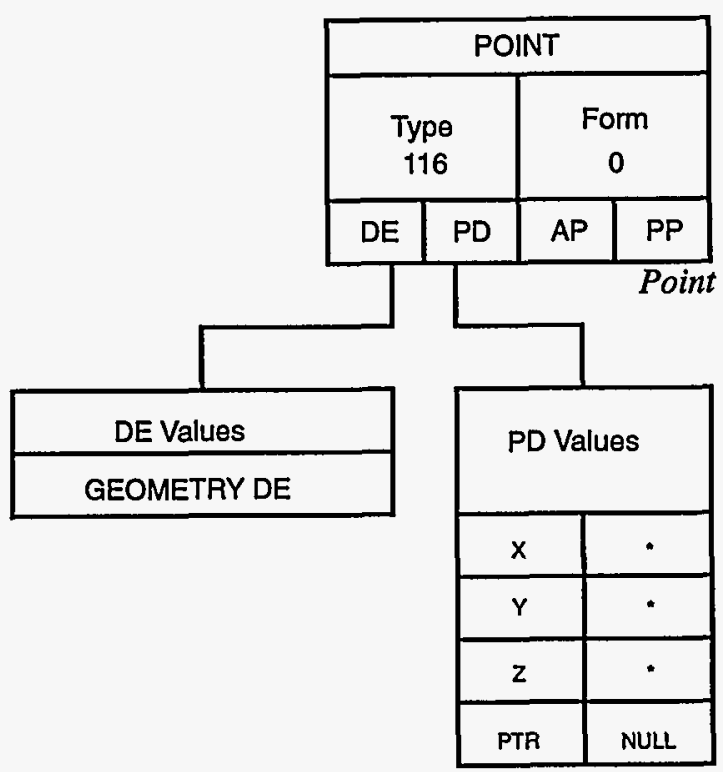




\subsubsection{Predefined Planar Shape}

\section{Description:}

The PREDEFINED PLANAR SHAPE object is a closed curve, which represents one of a set of predefined shapes, where the specific shape is defined by three floating point parameters. (This object is defined as the Flash Entity (Type 125); see IGES section 4.19).

\section{Requirements/Restrictions:}

\section{Translation Usage Notes:}

$5^{\text {General: }}$

Output:

Input: 


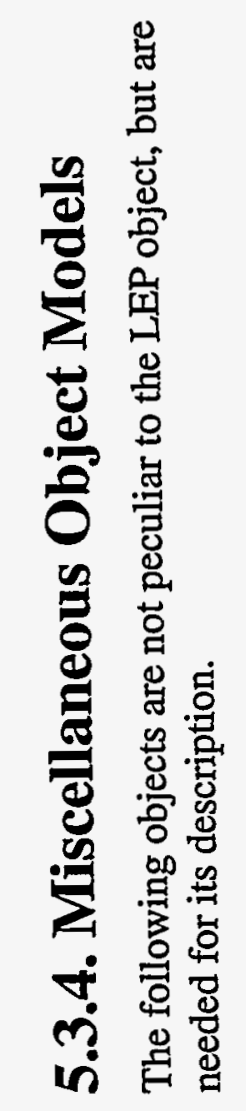




\subsubsection{Generic Data Property}

\section{Description:}

The GENERIC DATA PROPERTY object maps directly to the IGES Generic Data Property Entity (Type 406, Form 27). Please refer to IGES for additional information.

\section{Requirements/Restrictions:}

1. The GENERIC DATA PROPERTY object may be referenced from the Property Pointer list of any other object in the IGES model.

\section{๖ू Translation Usage Notes:}

Where possible, select the PD Name value from the predefined list beginning on the next page, allowing the receiving system to interpret the meaning of the Name string consistent with this AP.

General:

Output:

Input:

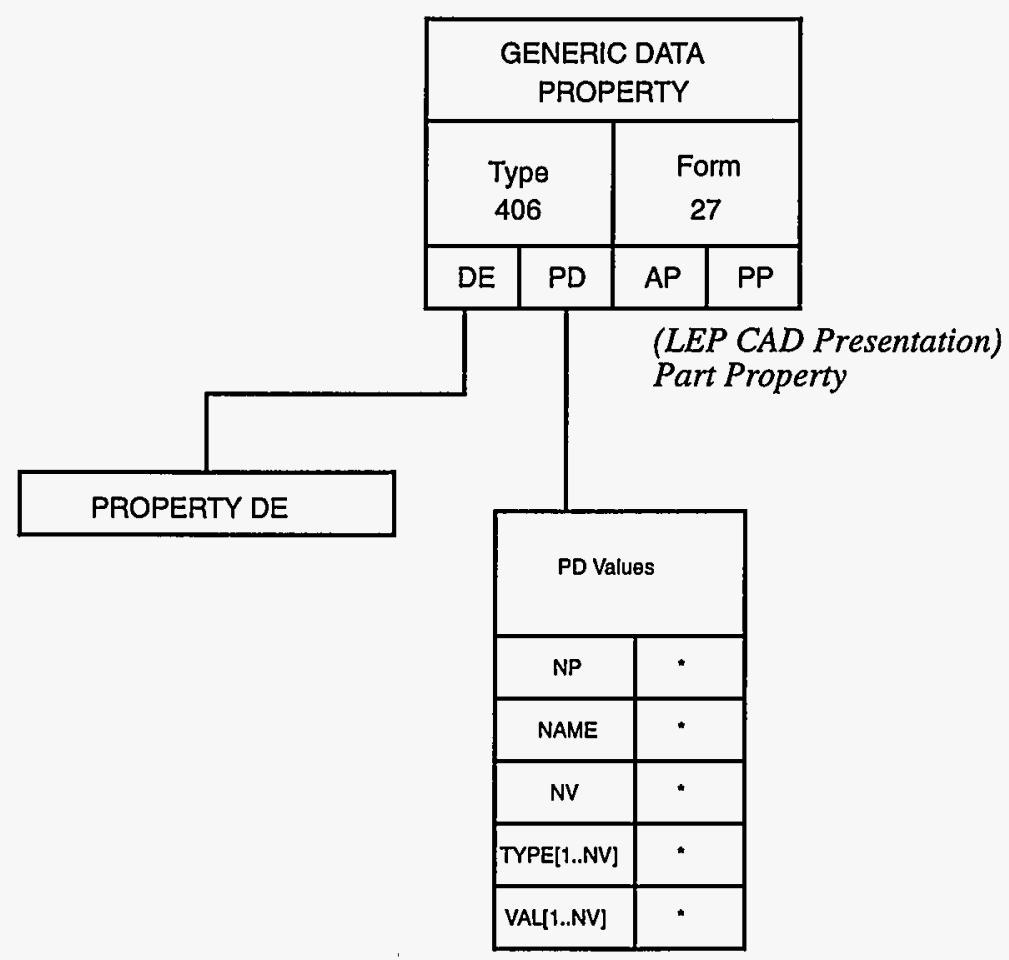




\section{Predefined values for the Generic Property "Name" parameter:}

abs_voltage_max The property (of the PACKAGE SYMBOL INSTANCE) which provides simulation and analysis programs an upper limit on the expected EMF to be applied to the component.

allowable_test_point The logical property (of a Connect Point Entity (Type 132) indicating it MAY be probed by the tester.

area The logical property of a JOIN object indicating that the surface bounded is conductive and is associated with a NET.

assembly_number The property (of the LEP PHYSICAL LAYOUT IGES FILE) which provides the text string name of the LEP assembly drawing file (assigned by the enterprise that created the LEP design).

component_default_padstack The logical property (of a PADSTACK DEFINITION) to indicate that nothing special has been added or removed from the Padstack selected to be generic.

component_height The length measure (of the PACKAGE SYMBOLDEFINITION) which provides insertion processes a $\mathrm{z}$ dimension measured from the nearer surface of the LEP below which the component shall reside after insertion (or an indication no value is provided).

component_insertable The logical property (of the $P A C K$ $A G E S Y M B O L$ DEFINITION) which provides insertion processes an indication as to whether the component lends itself to processing or not.

component_insertion_force A real number providing the maximum insertion force to be applied.

component_insertion_height $A$ length measure providing the maximum difference in the $\mathrm{z}$ direction between the nearer surface of the LEP and the farthest point, edge, or surface of the component.

component_insertion_origin The length measure (of the PACKAGE SYMBOL DEFINITION) which provides insertion processes the real $\mathrm{X}$ and $\mathrm{Y}$ offset from the definition space origin to the origin used by the inserter.

component_instance_side The text string (of the PACK$A G E S Y M B O L$ INSTANCE) which provides other processes a text string indicating on which side (top or bottom) of the LEP the component will be found.

component_keep_outline The logical property of a CLOSED CURVE in the $\mathrm{Z}=0.0$ plane flagging it as an $\mathrm{X}, \mathrm{Y}$ region of inclusion or exclusion of LEP Components.

component_layout_surface The text string(of the $P A C K$ AGE SYMBOL DEFINITION) which indicates which LEP surface the component defined occupies. \{ front/back/top/ bottom/both/unknown \}

component_outline_overhang The property (of the CLOSED CURVE) depicting the outline of a connector or other component which extends beyond the edge of the LEP outline.

component_max_size The property (of the PACKAGE SYMBOL DEFINITION) which provides insertion processes three maximum dimensions inside which the component can be contained.

component_physical_thickness The property (of the PACKAGE SYMBOL DEFINITION) which provides insertion processes with the depth space in which the component body is contained. 
component_pkg_type The textual property (of the $P A C K$ $A G E S Y M B O L D E F I N I T I O N$ ) which provides the package type name of the component defined.

component_placement_angle An angular measure providing the insertion machinery the rotation to be applied between grasp and insertion relative to the default component rotation.

component_placement_body A string providing a text code corresponding to the package type of the component to be inserted.

component_placement_center The length measures providing the insertion machinery the $\mathrm{X}, \mathrm{Y}$, and $\mathrm{Z}$ offset from the component origin to the origin presumed by the inserter.

component_placement_form_code An integer providing the insertion machinery an integer code to indicate the forming needed for the referencing component.

component_placement_form_code_description A string providing a text description of the forming needed for the referencing component.

component_placement_lead_diameter A length measure providing the insertion machinery the nominal diameter of the pin to be inserted.

component_placement_yn A logical property indicating whether the referencing entity models a component which is to be inserted or not.

component_placement_zspan A string providing a text code corresponding to the insertion machine depth stop.

component_preplace The property (of the PACKAGE SYM$B O L$ INSTANCE) which provides placement systems a predefined location and/or mounting side for that particular component instance. component_std_name The textual property (of the $P A C K$ $A G E S Y M B O L D E F I N I T I O N$ ) which provides the name of the component defined.

component_tech_type The textual property (of the $P A C K$ $A G E S Y M B O L D E F I N I T I O N$ ) which provides the name of the technology type (SMT or through-hole) of the component defined.

component_version The textual property (of the $P A C K$ AGE SYMBOL DEFINITION) which provides the (alphanumeric) version identifier of the component defined.

component_via The marking of the VIA instance to indicate it will be built around a component PIN.

conductive_trace_function The descriptive text property of an open curve defining a conductive JOIN on a particular layer or set of layers of an LEP intended to connect Pins, Pads and Vias to a power source, a ground return or a particular signal.

date The textual property (of an LEP) providing a value of the form $\mathrm{mm} / \mathrm{dd} / \mathrm{yy}$ plus a second value from the set \{file_creation, last_revision, revision_effective \}

fiducial The compound textual property (of an LEP CLOSED CURVE) to indicate its use as a positional reference. The first value indicates the nature of the owning entity. \{component/cluster/board/panel\} The second value indicates whether the locating symbol appears on the top or the bottom surface of the LEP. $\{$ top/bottom \}

jumper_wire The logical property of a JOIN object indicating that the object is conductive and is associated with a NET.

junction_max_t The temperature measure (of the $P A C K$ $A G E S Y M B O L$ INSTANCE) which provides simulation and analysis programs a value of the maximum temperature rating of the semi-conductor junction in the component. 
not_allowable_test_point The logical property (of a Connect Point Entity (Type 132) indicating it shall NOT be probed by the tester.

pad_tech_type The textual property (of the Connect Point Entity (Type 132) modeling the $P A D, P I N$, or socket acting as Port to the LEP or one of its components) which indicates the type of technology used in implementing the port.

panelThe logical property of a PANEL DEFINITION object indicating that the object is an LEP manufacturing item created to facilitate the fabrication of one or more PWBs.

panel_offset A pair of length measures providing the $\mathrm{X}, \mathrm{Y}$ offset from the LEP PHYSICAL LAYOUT IGES FILE to the origin of the fabrication panel.

pathThe logical property of a JOIN object indicating that the non-closed curve is conductive and is associated with a NET.

physical_outline The required property of a CLOSED CURVE surrounding the silhouette viewed in a plane parallel to a layer of the LEP.

physical_padstack_definition The logical property (of the set of entities depicting the sequence of PADs at a particular $\mathrm{X}, \mathrm{Y}$ location of an LEP) which tags the parent entity as the definition. Some of the $P A D$ s are to be connected to each other and to any conductor penetrating the hole at the $\mathrm{X}, \mathrm{Y}$ location. Other $P A D$ s may be intentionally isolated electrically from any conductor penetrating the hole at the $\mathrm{X}, \mathrm{Y}$ location.

pin1 The logical property (of the Connect Point Entity (Type 132) modeling the $P A D, P I N$, or socket acting as Port to the LEP or one of its components) which tags the port as "Pin 1" of the device.

placement_outline The orientational and positional property of a CLOSED CURVE indicating the intended position of a particular component on a physical layer of the LEP.

planar_shape_function The textual property of a CLOSED CURVE defining a conductive region of a particular layer or set of layers of an LEP intended to connect PINS, PADs and VIAs to a power source, a ground return or a particular signal.

pwb_number The textual property (of the LEP PHYSICAL LAYOUT IGES FILE defining the substrate) which provides the name of the LEP design drawing file (assigned by the enterprise that created the LEP design).

pwb_physical_outline The logical property (of an LEP CLOSED CURVE) which indicates the curve represents the physical outline of the LEP substrate.

schematic_number The textual property (of the LEP TECHNICAL ILLUSTRATION IGES FILE) which provides the name of the schematic drawing file (assigned by the enterprise that created the LEP design),

selected_test_point The logical property [of a Connect Point Entity (Type 132)] indicating it has been targeted to be probed by the tester.

silkscreen_bot The property indicating the referencing Text and/or graphics are to be applied to the Bottom Layer of the LEP.

silkscreen_top The property indicating the referencing Text and/or graphics are to be applied to the Top Layer of the LEP.

standalone_via The tagging of the VIA instance to indicate it will not be built around a component Pin.

station_action The textual property (of the SUBFIGURE INSTANCE representing the work station where some processing is to occur) which indicates what kind of processing is to be done. 
station_number A string providing the I. D. of the work station where some processing is to occur.

test_point_pwb_id The textual property (of a stand-alone $V I A)$ to provide a unique name to facilitate test descriptions.

test_point_nail_id The property (of a tester probe) to uniquely identify the probe to be put in contact with the referencing LEP feature.

therm_cond The textual property (of the Component Instance) which provides simulation and analysis programs a value of the nominal thermal conductivity coefficient (see IGES Tabular Data Property Entity (Type 406, Form 11), PTYPE=19) of the component case material.

therm_jc The property (of the Component Instance) which provides simulation and analysis programs a value of the nominal thermal resistance between the semiconductor junction and the outer case surface in watts per degree Centigrade.

therm_r The property (of the PACKAGE SYMBOL

INSTANCE) which provides simulation and analysis programs a value of the nominal thermal resistance from case to ambient in watts per degree Centigrade.

thermal_outline The graphic property of a CLOSED CURVE indicating the extent of thermal emission or sensitivity of the component(s) mounted in the vicinity.

tolerance The permitted range of the value referencing referencing this property.

top_level_assembly_instance The logical property (of the LEP entity) tagging it as the highest level instance within the scope of the model.

trace_bot The logical property of a JOIN indicating it appears on the Bottom layer of the LEP.

trace_keep_outline The property (of a CLOSED CURVE in the $\mathrm{Z}=0.0$ plane) defining an $\mathrm{X}, \mathrm{Y}$ region of inclusion or exclusion of LEP Traces.

trace_n The property of a JOIN indicating it appears on layer number $n$ of the LEP.

trace_top The logical property of a JOIN indicating it appears on the Top layer of the LEP.

trace_2 The property of a JOIN indicating it appears on layer number 2 of the LEP.

via The logical property of a JOIN object indicating that a hole is conductive and is associated with a NET.

via_keep_outline The property (of a CLOSED CURVE in the $Z=0.0$ plane) defining an $X, Y$ region of inclusion or exclusion of LEP VIAs.

wire_wrap The logical property (of the PIN) indicating the connection is wirewrapped.

gauge The numeric property (of the JOIN) indicating the wire gauge (usually 26 or 30 ; occasionally others)

wire_bondThe logical property of a JOIN object indicating that the object is conductive and is associated with a NET.

wire_color color of wiring for maintenance and ease of assembly

twisted_pair The logical property (of the JOIN) indicating multiple wires are twisted together for better signal integrity; signal names are usually assigned to sort together (e.g., reset_D+ and reset_D-) to designate pairing.

wrap_class The (optional) textual property (of the JOIN); values are "A," "B," "MODIFIED-B", indicating how many wraps around the post, and the "tightness" of wrap. 


\subsubsection{Hierarchy Property}

\section{Description:}

The HIERARCHY object maps directly to the IGES Hierarchy Property Entity (Type 406, Form 10). Please refer to IGES for additional information.

\section{Requirements/Restrictions:}

1. The HIERARCHY object may be referenced from the Property Pointer list of any object that references physically subordinate children.

\section{Translation Usage Notes:}

$\Xi$

General:

Output:

Input:

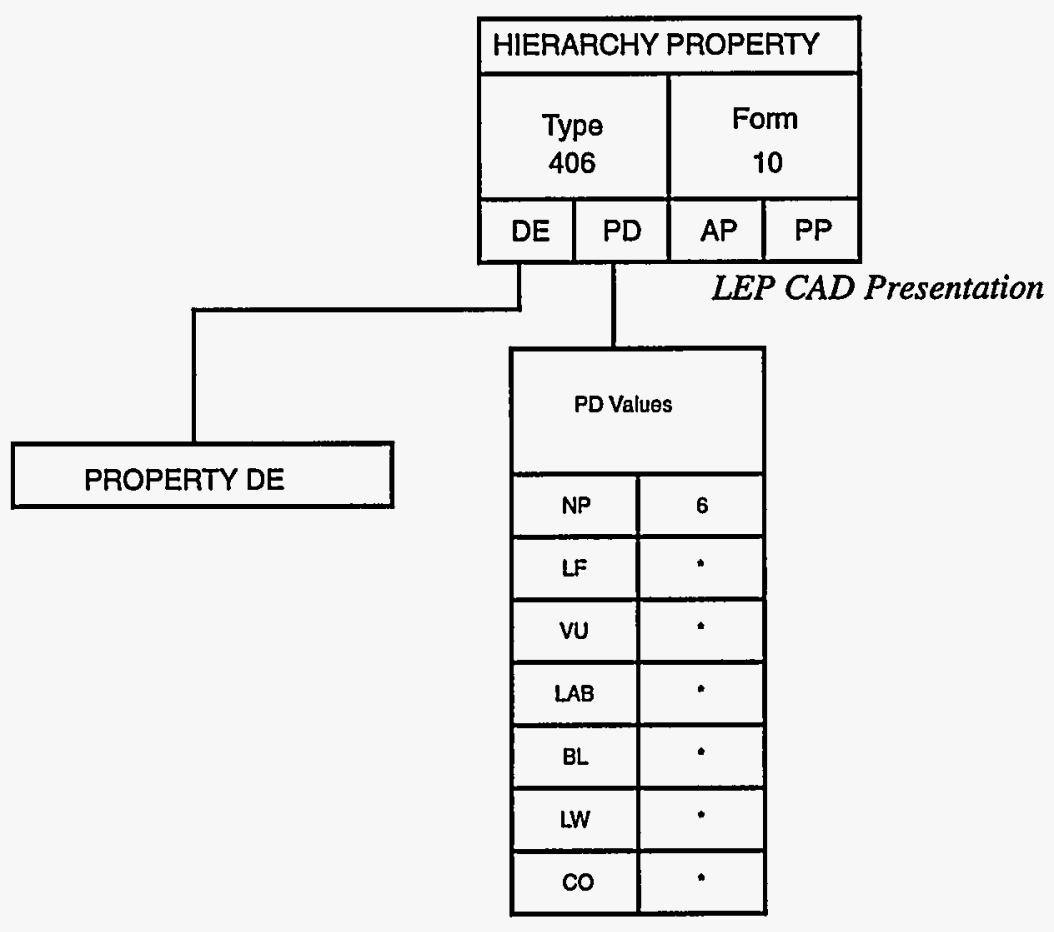




\subsubsection{Name}

\section{Description:}

The NAME object maps directly to the IGES Name Property Entity (Type 406, Form 15). Please refer to IGES for additional information.

\section{Requirements/Restrictions:}

1. The NAME object may be referenced by any other object that does not have an explicit name field in its parameter data.

2. The Name shall be unique within the scope of the file in which it appears.

怘

\section{Translation Usage Notes:}

\section{General:}

\section{Output:}

Input: 


\subsubsection{Network Subfigure Definition}

\section{Description:}

The NETWORK SUBFIGURE DEFINITION object maps directly to the IGES Network Subfigure Definition Entity (Type 320, Form 0). Please refer to IGES for additional information.

\section{Requirements/Restrictions:}

1. The NAME field in the Network Subfigure Definition Entity (Type 320) shall be unique (for all NETWORK SUBFIGURE DEFINITIONs of the same object type) within the scope of the IGES File.

$\vec{\omega}$

\section{Translation Usage Notes:}

\section{General:}

The values for the primary reference designator (PRD) and the pointer to the display of the primary reference designator (DPTR) are defaulted or set to null. Output:

Input:

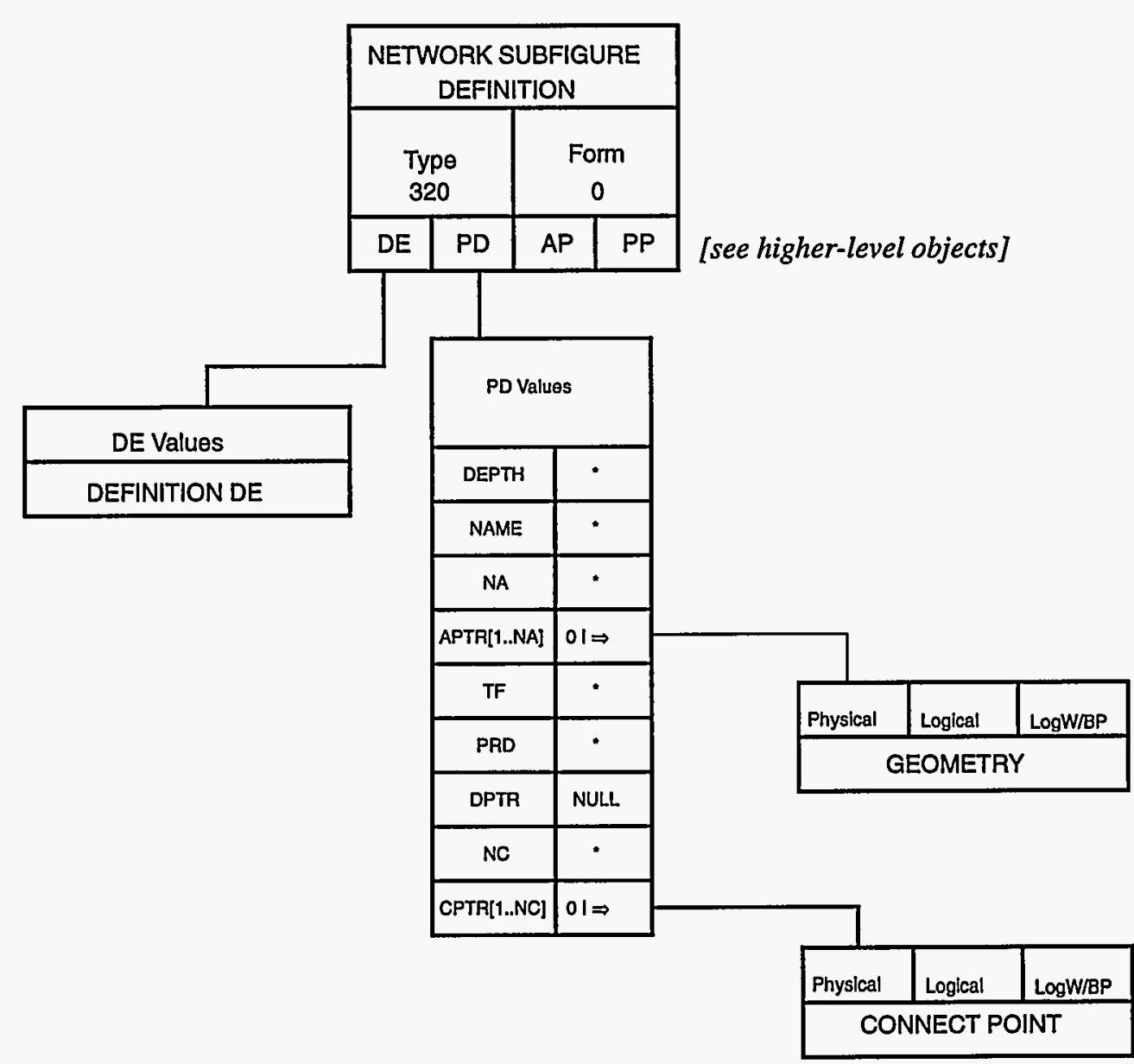

IGES graphic file: NETSFIGD 


\subsubsection{Network Subfigure Instance}

\section{Description:}

The NETWORK SUBFIGURE INSTANCE object maps directly to the IGES Network Subfigure Instance Entity (Type 420, Form 0). Please refer to IGES for additional information.

\section{Requirements/Restrictions:}

1. The reference designator shall be entered in the PD PRD (A TEXT STRING object shall not be used for the reference designator).

2. The pointer (PD DPTR) shall be to the DE of the Text $\vec{A}$ Display Template Entity (Type 312) for display of the primary reference designator(PD PRD).

\section{Translation Usage Notes:}

\section{General:}

\section{Output:}

Input:

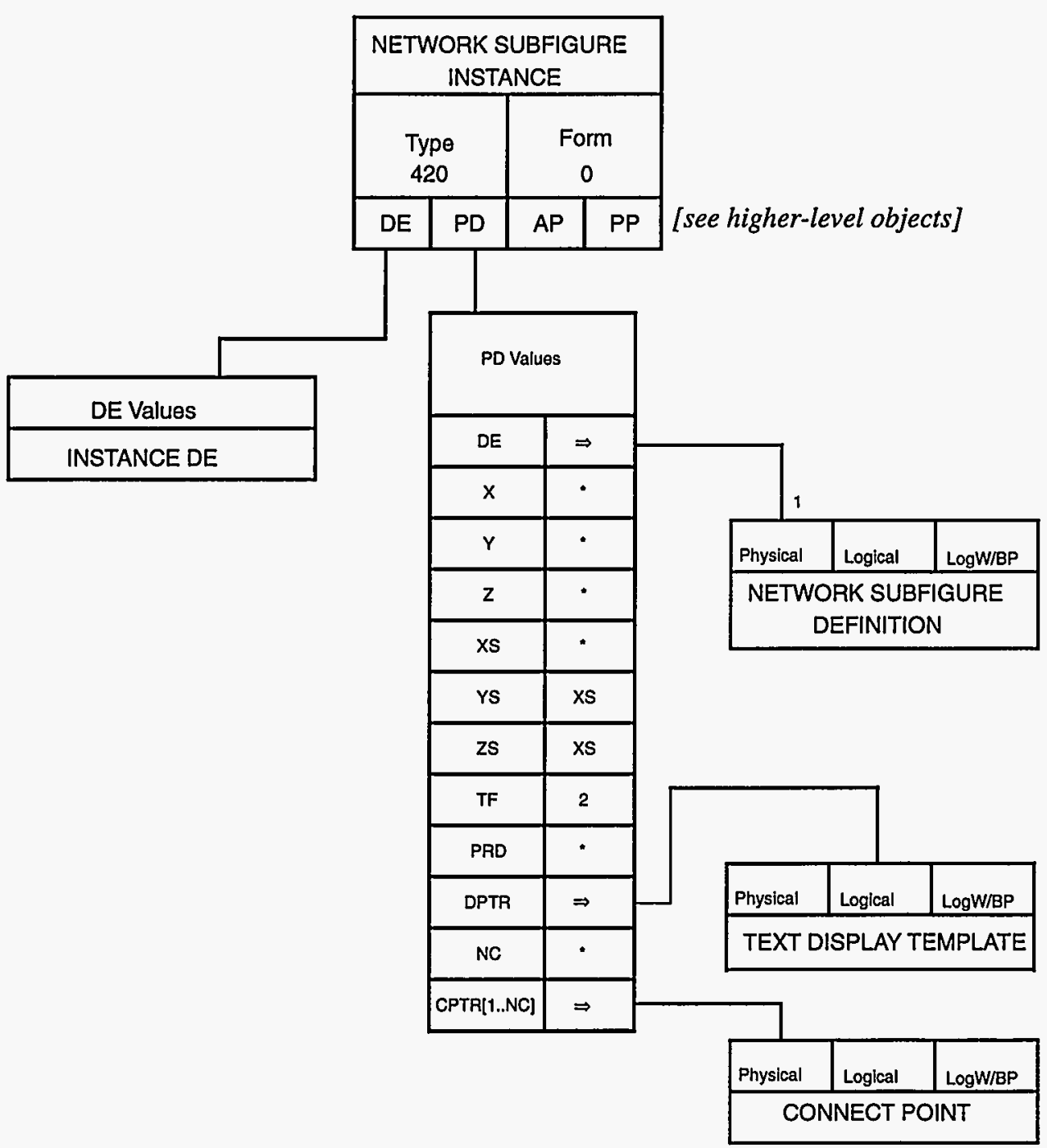

IGES graphic file: NETSFIGI 


\subsubsection{Object Locator}

\section{Description:}

The OBJECT LOCATOR object is any lesser object whcih documents location.

\section{Requirements/Restrictions:}

\section{Translation Usage Notes:}

General:

Output:

ज् Input:

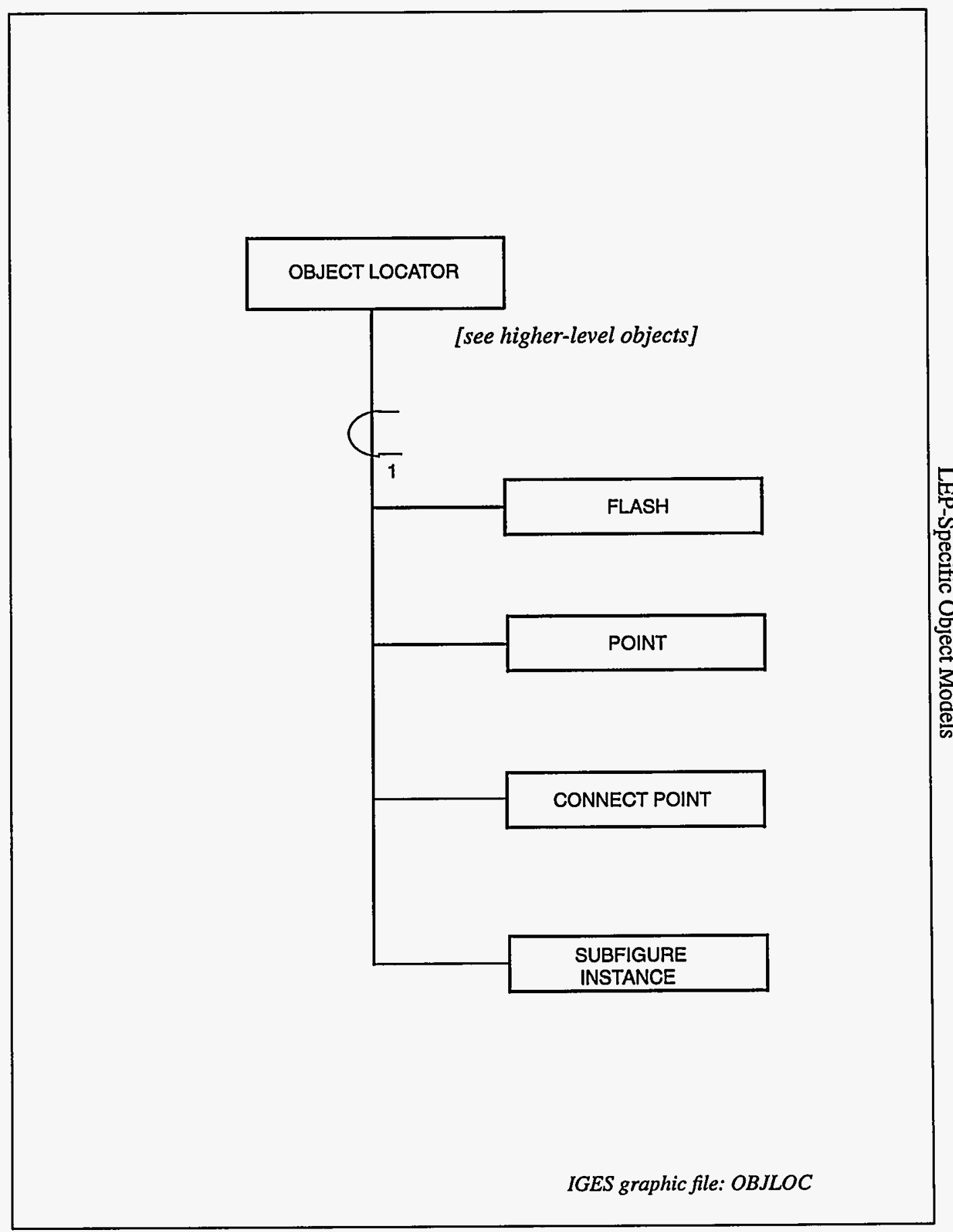




\subsubsection{Part Number Property}

\section{Description:}

The PART NUMBER PROPERTY object maps directly to the IGES Part Number Property (Type 406, Form 9). Please refer to IGES for additional information.

\section{Requirements/Restrictions:}

1. Shall attach to a Subfigure Instance Entity (Type 408) or a Network Subfigure Instance Entity (Type 420).

\section{Translation Usage Notes:}

One or more of four strings may be supplied as the generic part number (GPN), the Military Standard part

$\sigma$ number (MPN), the vendor part number or name (VPN), and/or the internal part number (IPN).

\section{General:}

\section{Output:}

\section{Input:}

PART NUMBER PROPERTY

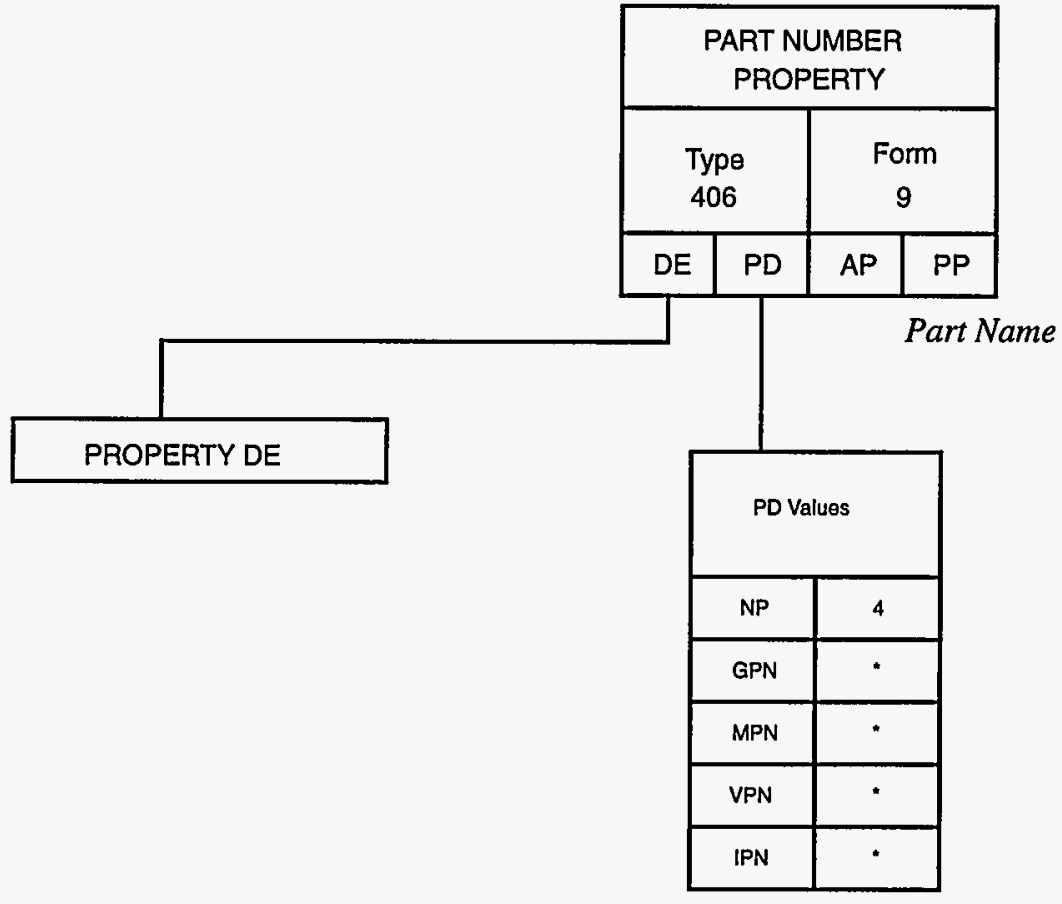




\subsubsection{Sectioned Area Fill Definition}

\section{Description:}

The SECTIONED AREA FILL DEFINITION object enables a CLOSED CURVE entity to be displayed with specific fill characteristics of solid or blank fill.

\section{Requirements/Restrictions:}

The SECTIONED AREA FILL DEFINITION may only be referenced by the following DISPLAY GEOMETRY objects which represent closed planar boundaries.

1. The following DISPLAY GEOMETRY objects may $\Xi \quad$ reference the Sectioned Area Fill Definition.
A) CLOSED CURVE
B) PREDEFINED PLANAR SHAPE
C) POLYGON

2. The fill pattern shall be code 6 or code 19 .

Translation Usage Notes:

General:

Output:

Input:

DE Values

PROPERTY DE
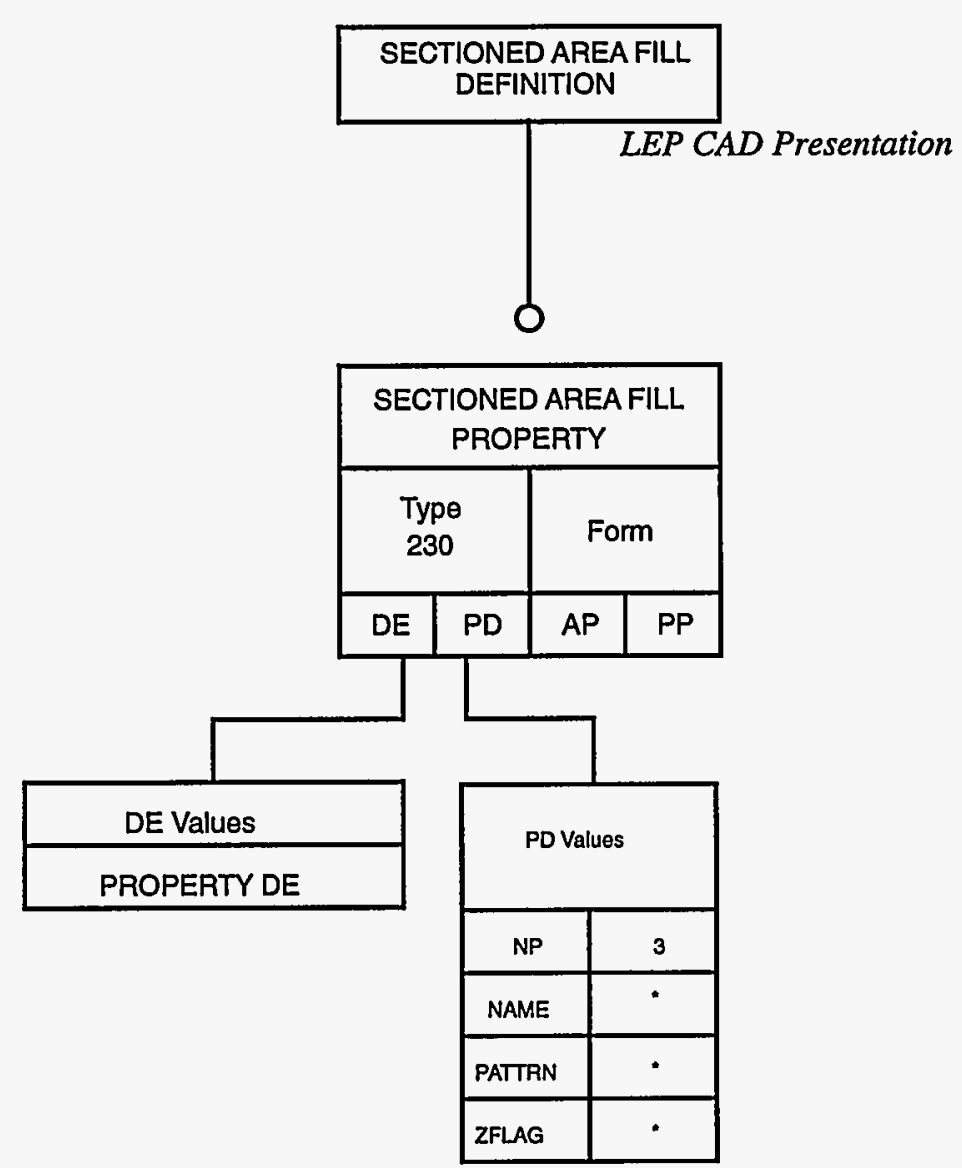


\subsubsection{Subfigure Definition}

\section{Description:}

The SUBFIGURE DEFINITION object maps directly to the IGES Subfigure Definition Entity (Type 308, Form

$0)$. Please refer to IGES for additional information.

\section{Requirements/Restrictions:}

1. The NAME field in the Subfigure Definition shall be unique (for all SUBFIGURE DEFINITIONs of the same object type) within the scope of the IGES File.

\section{Translation Usage Notes:}

$\vec{\infty}$

General:

Output:

Input:

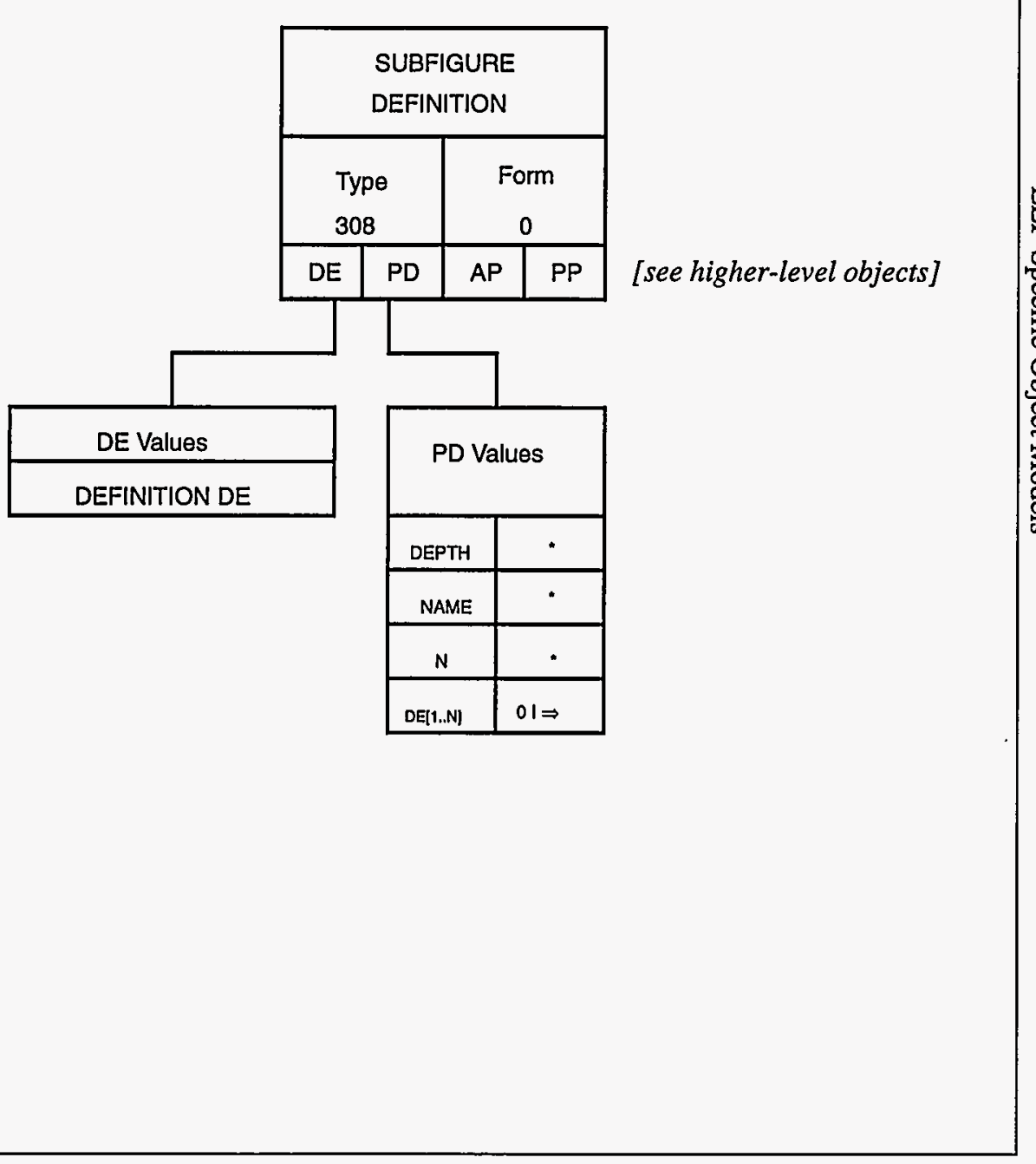




\subsubsection{Subfigure Instance}

\section{Description:}

The SUBFIGURE INSTANCE object maps directly to the IGES Subfigure Instance Entity (Type 408, Form 0). Please refer to IGES for additional information.

\section{Requirements/Restrictions:}

\section{Translation Usage Notes:}

\section{General:}

Б્ Output:

\section{Input:}




\subsubsection{Text String}

\section{Description:}

The TEXT STRING object is a General Note Entity (Type 212) containing one or more related character strings. This enables internal LEP annotation and provision of human interpretable semantics.

\section{Requirements/Restrictions:}

1. The General Note shall be Form $0,1,6,7$, or 8 .

2. The General Note FC field shall be one of $\{1,1001$, 1002 , or 1003 .

3. The General Note shall not be used to display

- The primary reference designator of a

Network Subfigure Instance Entity (Type 420),

- The pin name of a Connect Point Entity

(Type 132),

- The flow name of a Flow Associativity

Entity (Type 406, Form 18).

\section{Translation Usage Notes:}

\section{General:}

\section{Output:}

\section{Input:}

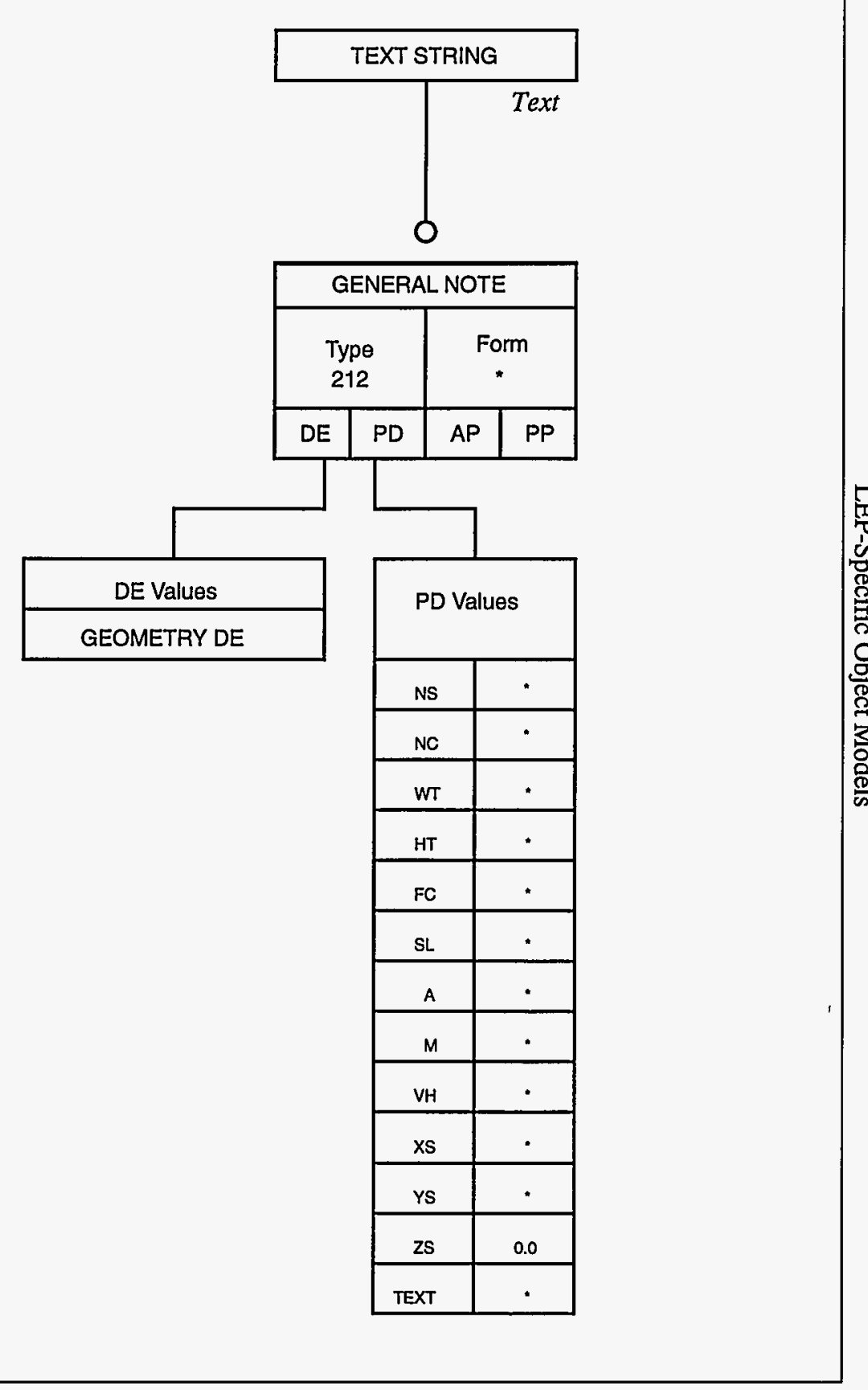




\subsubsection{Semantic Item}

\section{Description:}

The SEMANTIC ITEM describes the purpose for an IGES object in the context of the target system or application.

Requirements/Restrictions:

Translation Usage Notes:

General:

N Output:

Input:

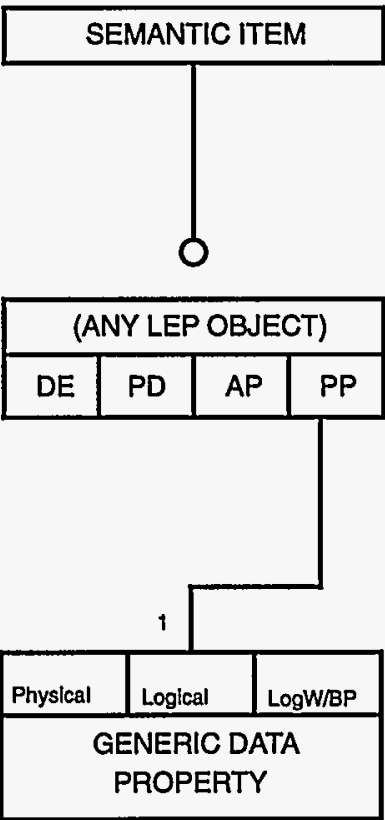



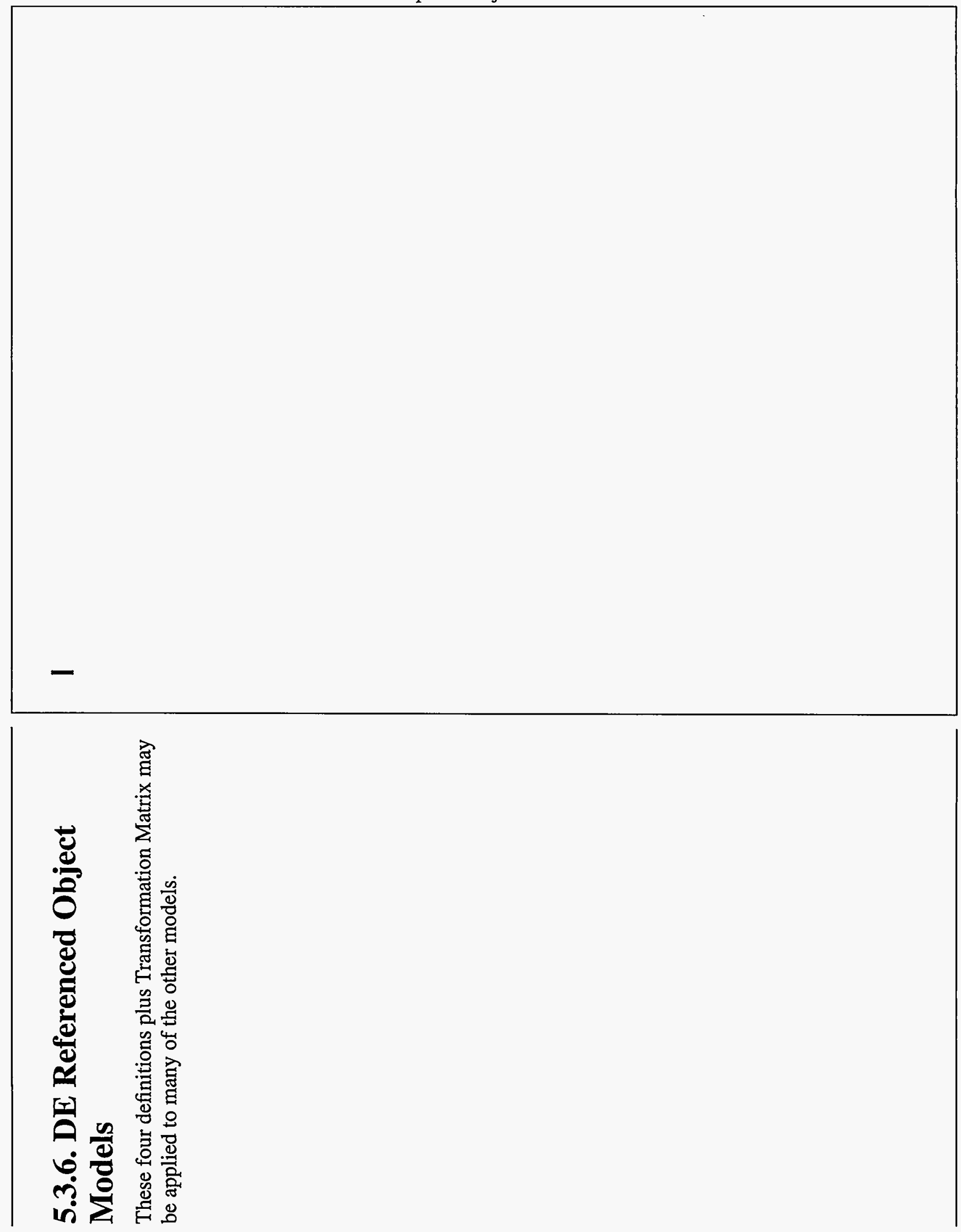


\section{| 5.3.6.1. Level Definition}

\section{Description:}

I The LEVEL DEFINITION object enables an entity to exist on one or more levels.

\section{Requirements/Restrictions:}

1. For the entity to be defined on a single level, it's DE field 5 shall contain that Level number.

I 2. For the entity to be defined on two or more levels, its DE field 5 shall contain a negated pointer to the appropriate Definition Levels Property Entity (Type 406, Form 1).

\section{$\underset{\mathcal{W}}{\mathbb{W}}$ Translation Usage Notes:}

\section{General:}

Output:

\section{Input:}

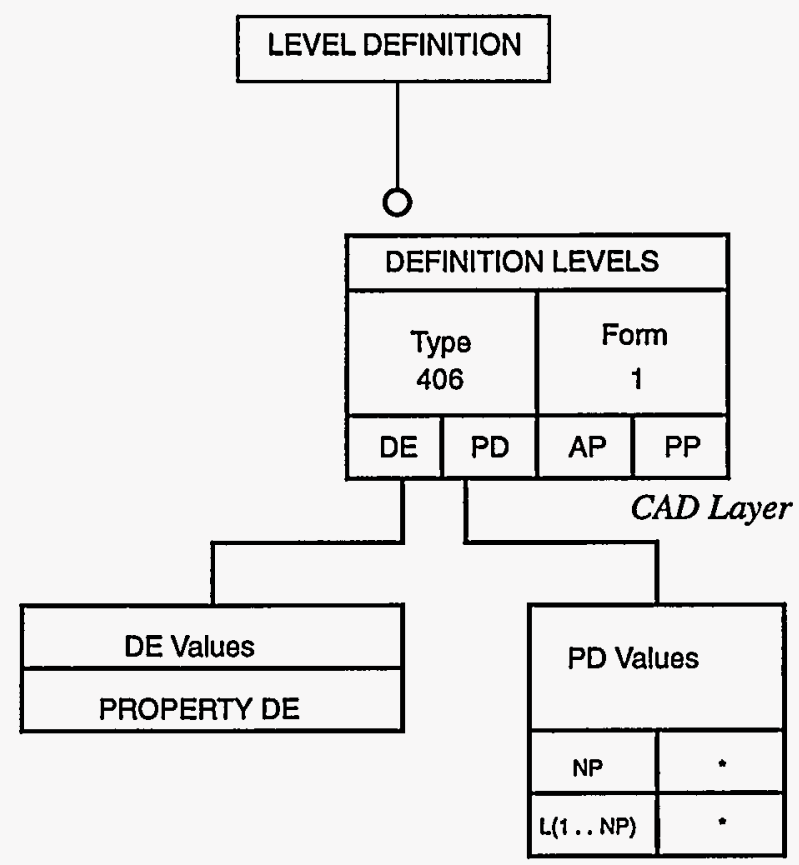




\subsubsection{Line Width Definition}

\section{Description:}

The LINE WIDTH DEFINITION object enables an entity to have specific line width characteristics which include width, justification, and specific end conditions.

\section{Requirements/Restrictions:}

1. The LINE WIDTH DEFINITION shall only be invoked by DISPLAY GEOMETRY objects.

Translation Usage Notes:

$\vec{\sim}$ General:

Output:

Input: 


\section{| 5.3.6.3. Transformation Matrix}

\section{Description:}

The TRANSFORMATION MATRIX object is either a Transformation Matrix (Normal) or a Transformation Matrix (Mirrored) Entity (Type 124).

\section{Requirements/Restrictions:}

1. The determinant of the matrix shall be equal to plus or minus one.

\section{Translation Usage Notes:}

\section{General:}

讪

Output:

Input:

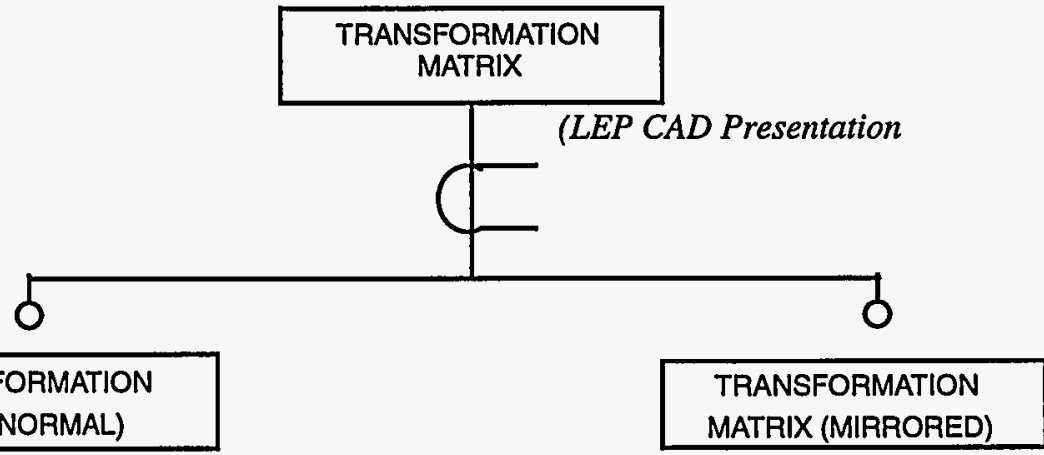


I 5.3.6.4. Transformation Matrix (Mirrored)

\section{Description:}

The TRANSFORMATION MATRIX (MIRRORED) object calls for an X,Y, Z rotation and an X,Y, Z offset in a left-handed coordinate system.

\section{Requirements/Restrictions:}

1. The determinant of the matrix shall be equal to plus or minus one.

\section{Translation Usage Notes:}

సু General:

\section{Output:}

Input:

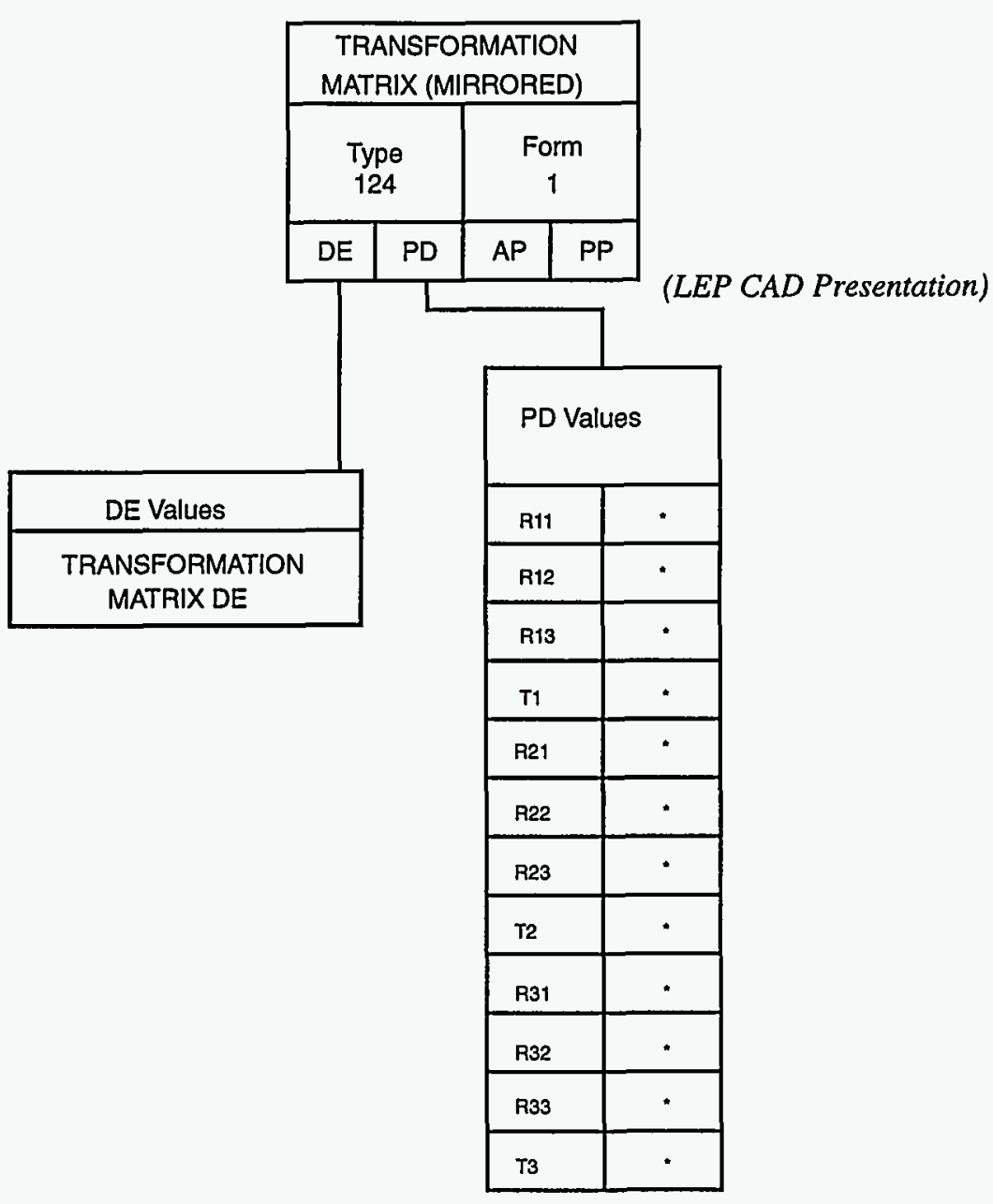




\subsubsection{Transformation Matrix (Normal)}

\section{Description:}

The TRANSFORMATION MATRIX (NORMAL) object calls for an $X, Y, Z$ rotation (and an $X, Y, Z$ offset) in a right-handed coordinate system.

\section{Requirements/Restrictions:}

1. The determinant of the matrix shall be equal to plus or minus one.

\section{Translation Usage Notes:}

స్ General:

\section{Output:}

Input: 


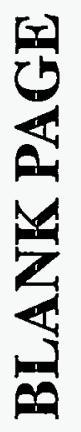




\subsubsection{DE Section Object Models}

Default values for the DE section are provided for seven classes of objects.

\subsubsection{Associativity DE}

\section{Description:}

The ASSOCIATIVITY DE object is an IGES Directory Entry Section template to be used by all associativity entities.

\section{Requirements/Restrictions:}

\section{్ㅠ Translation Usage Notes:}

General:

Output:

Input: 


\subsubsection{Definition DE}

\section{Description:}

The DEFINITION DE object is an IGES Directory Entry Section template to be used by all definition entities.

\section{Requirements/Restrictions:}

1. The Hierarchy Status shall indicate independence.

\section{Translation Usage Notes:}

\section{General:}

Output:

फे Input:

\begin{tabular}{|c|c|}
\hline \multicolumn{2}{|c|}{ DE Values } \\
\hline Entity Type & * \\
\hline Parameters & - \\
\hline Structure & $N / A$ \\
\hline Line Font & $N / A$ \\
\hline Level & $N / A$ \\
\hline Vlew & $\mathrm{N} / \mathrm{A}$ \\
\hline TM & $N / A$ \\
\hline LDis Assoc & N/A \\
\hline Blank & N/A \\
\hline Subord & - \\
\hline Entity Use & 02 \\
\hline Hierarchy & 01 \\
\hline Sequence & .. \\
\hline Entity Type & * \\
\hline Line Welght & N/A \\
\hline Color & VALUE \\
\hline Parm Count & .- \\
\hline Form number & * \\
\hline Reserved & -- \\
\hline Reserved &.- \\
\hline Label & N/A \\
\hline Subscript & $N / A$ \\
\hline Sequence & - \\
\hline
\end{tabular}




\subsubsection{Geometry DE}

\section{Description:}

The GEOMETRY DE object is an IGES Directory Entry Section template to be used by all display geometry entities.

\section{Requirements/Restrictions:}

1. The REGION FILL DEFINITION only applies to the CLOSED CURVE and the PREDEFINED PLANAR SHAPE objects.

2. The Line Width Definition only applies to geometry and Text String objects.

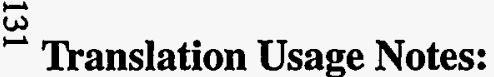

\section{General:}

\section{Output:}

Input:

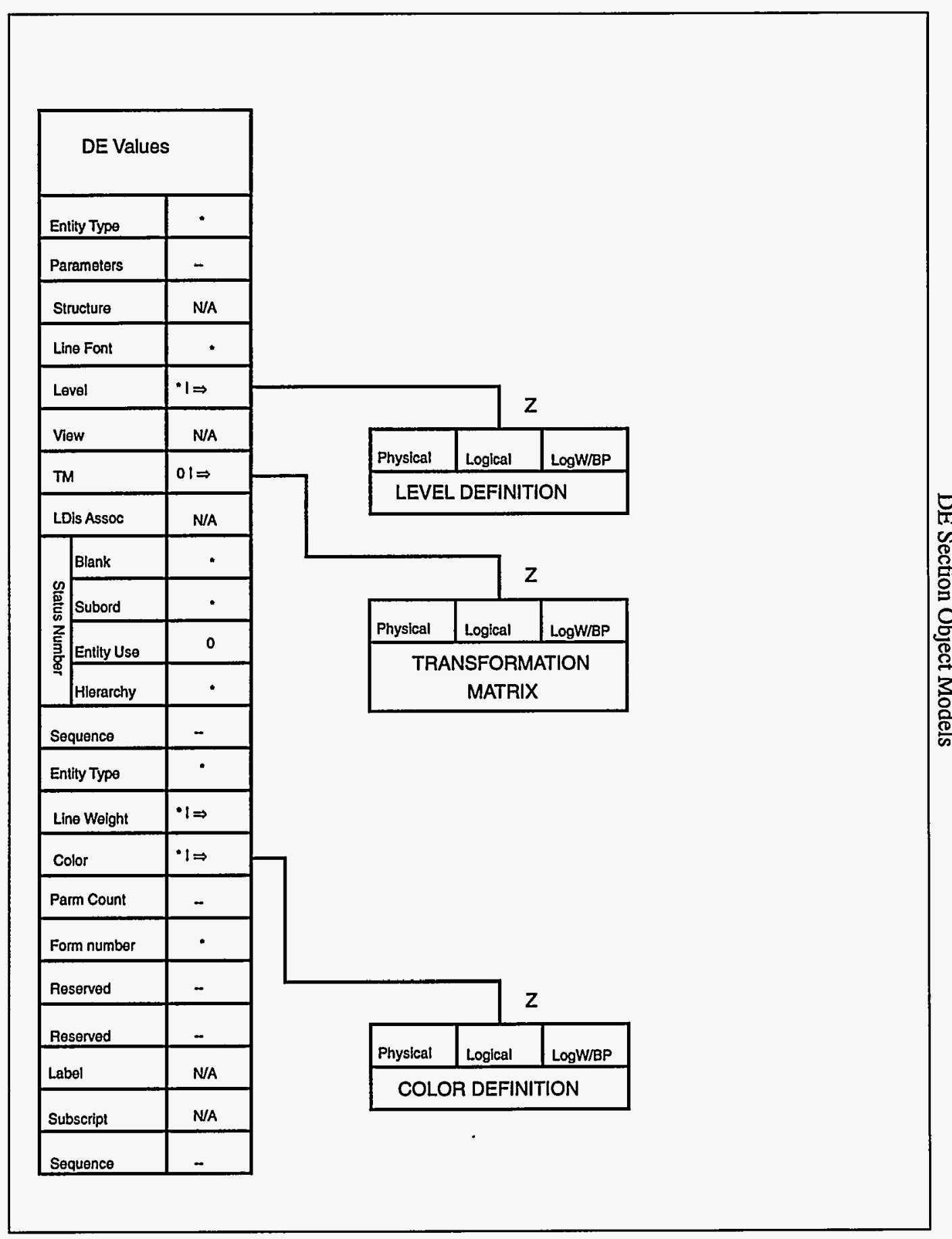




\subsubsection{Instance DE}

\section{Description:}

The INSTANCE DE object is an IGES Directory Entry Section template to be used by all instance entities.

\section{Requirements/Restrictions:}

\section{Translation Usage Notes:}

\section{General:}

\section{Output:}

岕 Input:

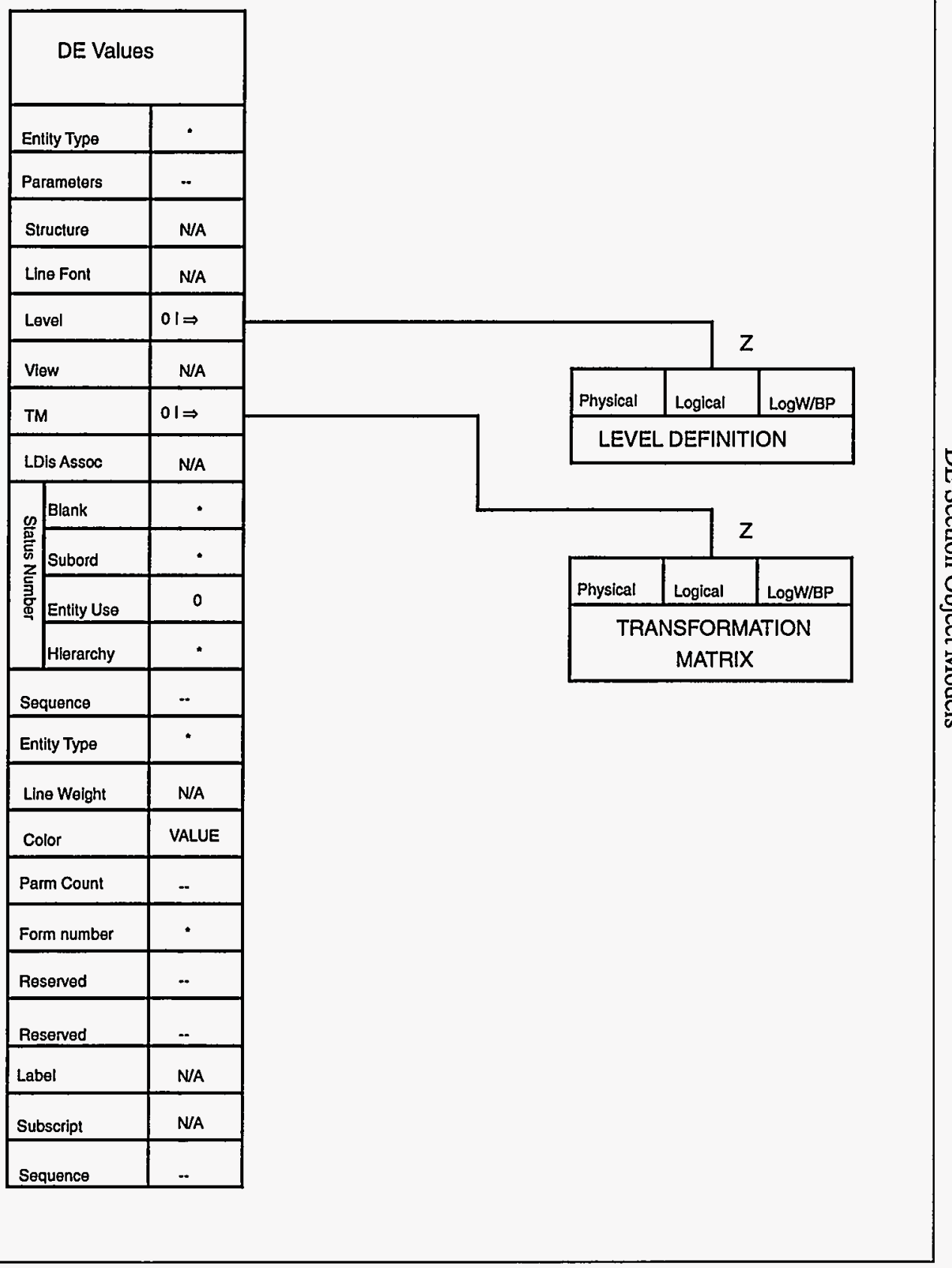




\subsubsection{Property DE}

\section{Description:}

The PROPERTY DE object is an IGES Directory Entry Section template to be used by all property and attribute entities.

\section{Requirements/Restrictions:}

1. For the Level to LEP LAYER MAP PROPERTY, SUB$\mathrm{ORD}=0$

Translation Usage Notes:

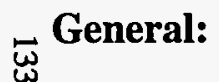

Output:

Input: 


\subsubsection{Transformation Matrix DE}

\section{Description:}

The TRANSFORMATION MATRIX DE object is an IGES Directory Entry Section template to be used by all Transformation Matrix entities.

\section{Requirements/Restrictions:}

Translation Usage Notes:

A Transformation Matrix can reference another Transformation Matrix.

\section{General: \\ $\stackrel{\omega}{\perp}$ \\ Output:}

Input:

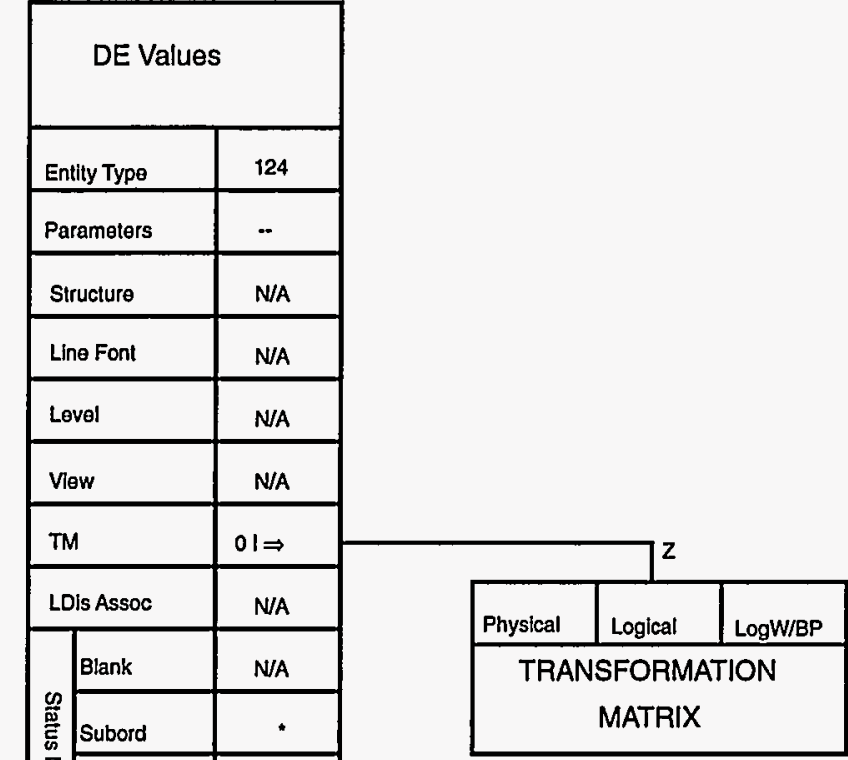

\begin{tabular}{|l|c|}
\hline Entity Use & 3 \\
\hline Hierarchy & N/A \\
\hline Sequence & - \\
\hline Entity Type & 124 \\
\hline Line Weight & N/A \\
\hline Color & N/A \\
\hline Parm Count & - \\
\hline Form number & 011 \\
\hline Reserved & - \\
\hline Reserved & -- \\
\hline Label & N/A \\
\hline Subscript & N/A \\
\hline Sequence & -- \\
\hline
\end{tabular}




\section{Blank Page}

(END OF SECTION 5) 


\section{Blank Page}




\section{IMPLEMENTATIONAND CONFORMANCE TESTING GUIDELINES}

The successful exchange of information using an IGES AP requires the participating organizations to establish information configuration control and software configuration control procedures for their product data creation and exchange systems. It must be understood that the use of IGES AP's will in many cases require organizations to revise their policies and procedures for the creation, exchange, and archival storage of product data. The successful use of an IGES application protocol also requires that the participating IGES processors conform to the AP specification. The purpose of conformance testing is to increase the confidence that different implementations of the AP will be able to exchange information successfully. This AP requires that the functionality of the LEP constructs be preserved in the translation into and out of the IGES format. Therefore, the CAD system for which the processors are being tested must provide this minimum level of functionality for modeling electronic product design and fabrication support systems. This section will define both testing requirements and guidelines for implementation of the functionality defined in the AIM section.

\subsection{PROCESSOR CONFORMANCE REQUIREMENTS}

The conformance requirements for implementations of this AP are enumerated as follows: 1.) All IGES files created by an AP compliant preprocessor shall conform to the IGES specification, PRO-100 and to the constructs specified in this AP. An AP compliant preprocessor shall convert LEP information of the ARM into the IGES constructs specified in the AIM, with all the required attributes and values. The functionality defined for each construct of the ARM shall be preserved. 2.) An AP compliant postprocessor shall interpret files that conform to the IGES constructs specified in this AP. It shall also convert each construct of the AIM into native constructs which match the geometry, attributes, and relationships of the LEP constructs specified in the ARM. The functionality of the LEP constructs shall be preserved.

Development and use of the IGES AP Abstract Test Suite is divided into several Test Groups (TG). Each test group contains discrete Test Purposes (TP). A TP defines the objective of an abstract test case. For a preprocessor, the TP begins with "to test the generation of a(n)...". For a post processor the TP begins with "to test the interpretation of a(n)...". An abstract test case is required for the preprocessor and postprocessor. An abstract test case is derived from a TP and is written in a formal language. When parameter values are provided for the constructs in the abstract case, it can be used to generate an executable test case. An abstract test case contains: TP; test case identifier; reference to specific parts of the AP; definition of constructs required to exercise the TP; statements indicating the construction sequence; and verdict criteria. Abstract test cases are documented in non-system specific procedures and are used to produce comparable results from the conformance testing of multiple implementations.

An executable test case is derived from an abstract test case and is in a form which allows it to be run on the implementation under test. An executable test case contains some or all of the following; TP; test case indentifier, reference to specific parts of the AP; constructs required to exercise the TP together with their associated parameter values; a test script defining the construct sequence; verdict criteria; an IGES file for postprocessor conformance testing; and a pictorial rep- 
resentation of the populated constructs.

\subsubsection{Testing Considerations}

In any situation where there is more than a modicum of information to represent, more complex structures are made from combining less complex structures in a very well defined and specific manner. The depth of such nesting is inconsequential, the relationships between the separate parts at a given nesting level, and the discrete assemblies at adjacent levels, are of prime importance. The IGES Electrical Application Committee defines the minimum level of intelligence as being able to support the concept of connectivity between two or more individual connection points on one:or more individual components using a unique path that connects only these specified objects. Further, each unique path has a name, as does each pin on each component. This functionality is defined in IGES 3.6.3 and is crucial for CALS (MUL-D-2800 Class III).

Each of these constructs is an organized collection of simpler ${ }^{1}$ items, the highest construct may be a fully populated $\mathrm{PCB}$, the lowest item is an individual entity. For example; several individual Line entities may be collected together using a signal list associativity ${ }^{2}$. The signal list associativity may have a name of "reset." The Lines it points to may each have their own properties, such as Line Widening (trace thickness). Each Line may be on a different level, with the thickness of the lines on the inner levels being different from those on the outer levels. These single individual . Lines, with their own properties become one item at the next level of abstraction. A PCB via keepout is simply a collection of contiguous lines or closed curves gathered together with an associativity which has a property of "board via keepout."

\subsubsection{Conformance Considerations}

The Layered Electrical Product Application Protocol defines how different functional constructs are represented. This eliminates the confusion and ambiguity that may result when there is more than one way to do something. However, files may conform to the AP, yet not include every entity in the AP. Conformance simply says that the information in the conformant file is represented using the AP defined entities, in the AP defined manner, so as to get the most information possible exchanged.

For example, a CALS Class III IGES file may well conform to the AP even though it does not contain a thermal outline for each component. It does not conform to the AP if all of the components are represented with simple Subfigure Definition and Instance entities, even if there is a thermal outline included in the subfigure definition. Yet a conforming file may well contain simple Subfigures, indeed, the padstacks may be represented with them.

Determining conformance is not as easy as just looking for the existence or absence of certain entities. Even assuming that all of the right entities are there, they may not be hooked up correctly, and simply noticing that a key entity, such as the signal list (called Flow Associativity in IGES) is missing doesn't mean it is not compliant.

All of the extraction algorithms presented are only valid for LEP AP files. They assume that each

1. Simple in terms of assembling the final product; recognizing that a bare chip integrated circuit, often the "starting point" of a product, may be the most complex LEP assembly component in terms of design.

2. The name of the actual IGES entity here called "signal list" is an IGES "Flow Associativity." The concept of connectivity is shared by both the electrical application, and the piping application. 
component has a reference designator as part of it's make up, or that a signal may be followed through the paths on the board, to each separate connect point (which has a pin identifier), which is part of a unique component instance.

While a MIL-D-28000 Class I picture looks just like a Class III picture, the lack of intelligence of the Class I file precludes most process automation and machine controlled functions. It is just a picture, and as such, only worth about 1,000 words.

Due to the complexity of this AP, it is not feasible to conduct exhaustive testing of processors for all possible combinations of AP constructs. The conformance testing requirements described in this section cover all constructs of the ARM. The enumerated test groups ( $\sec 6.2$ ) are not exhaustive (i.e., they do not cover all possible combinations of ARM and AIM constructs) although the commonly encountered information required for transfer indicated in Figure 6-1 is present.

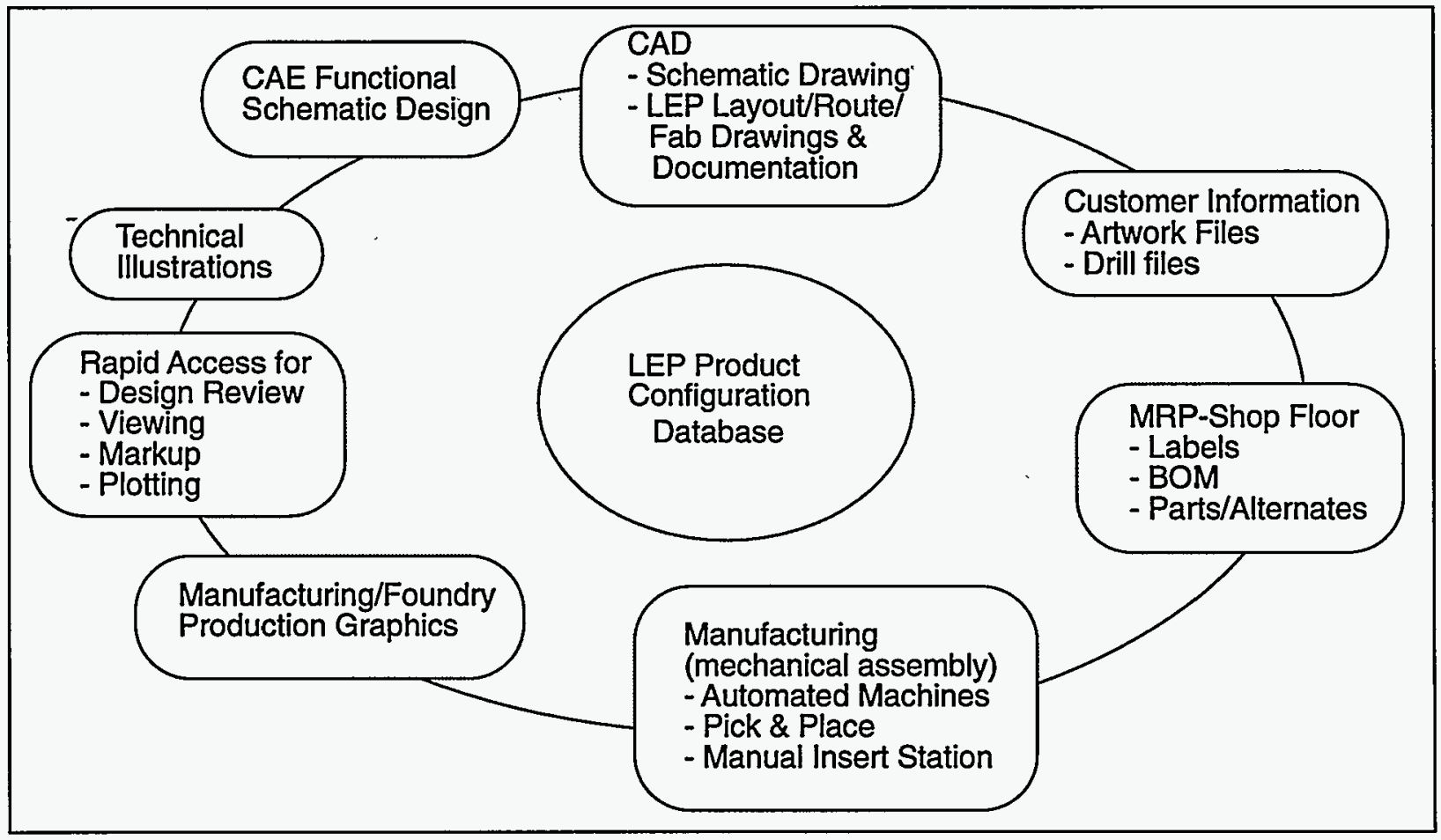

Figure 6-1, LEP Information Interface Requirements

\subsection{Test Purposes and Test Groups}

to test the generation of $a(n)$

to test the interpretation of $a(n)$

LEP Product Model Test Groups:

Test Group 1: Geometry, open and closed.

Test Group 2: Electrical and non-electrical symbols.

Test Group 3: Padstacks.

Test Group 4: Pads, Through-Hole pins, Vias and Testable Points.

Test Group 5: Electrical Component Definition. 
Test Group 6: Electrical Component Instance.

Test Group 7: Stand-alone Vias, Pads.

Test Group 8: Netlist.

Test Group 9: LEP PWB.

Test Group 10: Photolithograhy.

Test Group 11: LEP Fabrication.

Test Group 12: Machine Controlled Processes.

Test Group 13: Production Test.

LEP Special Uses Test Groups:

Test Group 14: Non-electrical Part.

Test Group 15: Panel.

Test Group 16: Production Engineering.

Test Group 17: Technical Publication.

Test Group 18: Dimensions.

Test Group 1: Geometry, open and closed.

Open Geometry is used for ordinary graphics, and electrical Traces. Closed Geometry is used for both Outlines and filled areas.

Test Purposes:

1. Point with no display symbol.

2. Two Point Line, 1.0 inch long.

3. Two Traces, each being 1.0 inch long and 0.010 inches wide and having two Points. The two Traces shall each have one end Point in common with the other. Each Trace shall be on a different Level.

4. Four line segments that define a closed, coplanar square shape (PLEASE NOTE: this is also known as a "Closed Curve" or an "Outline").

5. Grouping of four line segments and four 90 degree Arcs that define a square closed area with 0.25 inch radius rounded corners such that they are treated as a single entity (selected, moved, deleted).

6. Simple filled area. Grouping of four line segments that define a closed area such that they are treated as a single entity and filled with a solid fill.

7. Complex filled area. A filled area in the shape of an upper case character " $T$ ". Each area shall be longer than 2.0 inches, and wider than 1.0 inch. All corners shall be rounded with 0.25 inch radius Arcs.

Test Group 2: Electrical and non-electrical symbols. 
Test Purposes:

1. Simple filled area 0.120 inch extents; a circle, a square, a diamond.

2. Complex filled area 0.120 inch extents; a bow-tie, a bulls-eye.

3. Simple filled area 0.240 inch extents; a circle, a square, a diamond.

4. Fiducial.

Test Group 3: Padstacks.

Test Purposes:

1. Two 0.060 diameter circular conductive areas on two distinct Levels.

2. Two 0.048 diameter circular conductive areas on two other distinct Levels.

3. A closed square Outline with the Geometry being 0.010 inches wide, and 0.100 inch center to center spacing.

4. A 0.046 diameter drilled Hole, passing through the entire LEP, plated to 0.036 diameter.

5. A display symbol, that provides visual identification of the drilled Hole. This symbol shall be on yet another distinct Level.

6. The grouping of items 1-5 such that they appear as 1, addressable.... (PLEASE NOTE: this group is called a "Padstack").

Test Group 4: Pads, Through-Hole Pins, Vias and Test Points.

Test Purposes:

1. 0.060 square surface mount Pad.

2. 0.060 square surface mount Pad that may be used as a Test Point.

3. Padstack that is a Component Pin.

4. Padstack that is a Component Pin that may be used as a Test Point.

5. Padstack that is a Via that may be used as a Test Point.

Test Group 5: Defined Electrical Component:

Test Purposes:

1. Closed Curve defining the $2 \mathrm{D}$ physical Outline of the Component.

2. Closed Curve defining the 2D thermal Outline of the Component.

3. Closed Curve defining the 2D LEP PWB graphics Outline of the Component. (i.e. the Outline on the board; the "silk screen" graphics).

4. A Component of a specific package type (dip, sip, axial,...).

5. An SMT Component.

6. A Through-Hole Component. 
7. The height of the Component.

8. A restriction confining placement to one side only.

9. A restriction that the Component may not be auto-inserted or placed.

10. An association of a Component Pin/Pad to Component.

11. The Padstack referenced by Pin 1.

12. The Padstack referenced by other Pins.

(PLEASE NOTE: may be more than 2 types- quad has 4).

13. All Pins/Pads that are part of this Component.

14. The $\mathrm{x}, \mathrm{y}$ location (relative to Component origin) of pin1.

15. The $x, y$ location (relative to Component origin) of other Pins.:-

16. The alphanumeric tag of each Pin/Pad occurrence.

17. A restriction that a specific Pin/Pad may not be used as a Test Point. (while the Pin/Pad itself may be used as a Test Point, it may be disallowed when packaged due to problems like clearance or neighboring components.

Test Group 6: Placed Electrical Component.

Test Purposes:

1. Component with a unique reference designator.

2. The $x, y$ location of a Component.

3. The part number of a Component.

4. Association of the placed Component to the defined Component.

5. Component which must be inserted manually.

6. Component on the bottom side of the LEP PWB.

7. Component on the top side of the LEP PWB.

8. An association of a Pin/Pad to its parent Component.

Test Group 7: Stand-alone Vias, Pads.

Test Purposes:

1. Through-pin as a stand-alone Via not related to a Component.

2. The $x, y$ location of a stand-alone Via.

3. The alphanumeric identifier of a stand-alone Via occurrence.

4. The signal connected to a stand-alone Via occurrence.

5. Restriction that the Via may not be used as a Test Point.

6. Pad as a stand-alone object not related to a Component.

7. The $x, y$ location of a stand-alone Pad occurrence. 
8. Alphanumeric identifier of a stand-alone Pad occurrence.

9. Signal connected to a stand-alone Pad occurrence.

10. Restriction that the Pad occurrence may not be used as a Test Point.

Test Group 8: Netlist.

Test Purposes:

1. Association of all Pins that share a specific signal.

2. Association of all Pads that share a specific signal.

3. Association of all Vias that share a specific signal.

4. Association of all Test Points that share a specific signal.

5. Association of all interconnect Geometry, such as Traces, jumpers, and filled planes that share a specific signal.

6. Signal name.

7. Association of all objects (Pins, Pads, Vias, Test Points, and interconnect Geometry) to a signal name.

Test Group 9: LEP PWB.

Test purposes:

1. The physical Outline of the PWB.

2. The origin of the PWB.

3. The Outline(s) defining Component Keepin/Keepout regions.

4. The Outline(s) defining Trace Keepin/Keepout regions.

5. The Outline(s) defining Via Keepin/Keepout regions.

6. The Outline(s) defining conductive planes.

7. Default Via.

8. Default Padstack.

9. Padstack that is a Via, and it may be used as an allowable Test Point.

10. SMT Pad that may be used as an allowable Test Point.

11. Alphanumeric identifier such as "Assy 123-456-7890.rev2.1"

12. Three master registration fiducials, one of which is at the origin of the LEP PWB. The symbols shall be unique in appearance, and associated with each other.

13. Three fabrication fiducials, one of which is at the origin of the LEP PWB. The symbols shall be unique in appearance with respect to all other depictions in the file. The symbols shall be associated with each other. 
Test Group 10: Photolithograhy.

Test Purposes:

1. Single two Point Trace that is 1.0 inch long and 0.010 inches wide. The Line ends shall be round, and the 1.0 inch length shall be measured from the center of the Arc defining the round Line ends. (tip/tip length $=1.010 \mathrm{inch}$ ).

2. Single two Point Trace that is 1.0 inch long and 0.025 inches wide. The Line ends shall be square.

3. Two linear Traces 0.025 inches wide, each of which has two Points. One end Point of each Trace shall be connected to the other with an arc so that the lines are 60 degrees apart.

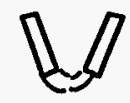

4. Three sets of three square filled shapes. The first set shall be 0.10 inch on each side, and 0.40 inches, center to center, away from each other. The second set shall be $0.070 \mathrm{inch}$ on each side, 0.30 center to center spacing, and the last set shall be 0.040 inch square, 0.20 inch spacing.

5. Three sets of two circular filled shapes. The first set shall be 0.10 inch diameter and 0.40 inches, center to center, away from each other. The second set shall be 0.070 inch diameter, 0.30 center to center spacing, and the last set shall be 0.040 inch diameter, 0.20 inch spacing.

6. Two sets of three fiducials, with different graphics appearance for each set. Each set shall occur on all masters.

7. The orientation of each master (rotated, flipped, mirrored).

Test Group 11: LEP Fabrication.

Test purposes:

1. Buried Via.

2. Unplated tool Hole with a diameter of 0.125 inch.

3. A complex cutout. For example, one that looks like a keyhole. The diameter of the circular part of the cutout shall be 0.400 inch. The width of the straight line portion shall be 0.200 inch. The total length shall be 1.0 inch.

4. The order of each master in the lay-up sequence.

5. Fiducials used for position of stratum registration.

Test Group 12: Machine Controlled Processes.

Component pick/place; insertion; verification.

Test purposes:

1. Identifier at the maximum coordinate along each side.

2. All Components that are between 1.0 and 3.0 inches along one edge of the LEP PWB, and 0.0 and maximum board size along the other edge. 
3. The $x, y$ location of the Component center.

4. The $x, y$ physical size of the Component ( $\mathrm{x} / \mathrm{y} \min / \mathrm{max}$ ).

5. The rotation of the placed Component with respect to the Component definition.

6. The lead span of a leaded, auto-insertable Component.

7. The lead diameter of a leaded, auto-insertable Component.

8. Insertion force required for a leaded, auto-insertable Component.

9. The $x, y$ location of pin1 (used in verification).

Test Group 13: Production Test; PWB.

Note that each signal should have at least one Test Point, preferably an assigned Test Point.

Test purposes:

1. Padstack that is a Component Pin, and is an allowable Test Point.

2. Padstack that is a Component Pin, and is an assigned Test Point.

3. Padstack that is a Via, and is an allowable Test Point.

4. Padstack that is a Via, and is an assigned Test Point.

5. Pad that is an allowable Test Point.

6. Pad that is an assigned Test Point.

7. A unique identifier for each Test Point.

8 The $x, y$ location, relative to the origin of the LEP PWB, of each assigned Test Point.

9. The signal name at each assigned Test Point.

10. A tester assigned identifier for each assigned Test Point.

11. Electrical properties, such as voltage, amperage, frequency.

Test Group 14: Non-electrical part.

Test purposes:

1. The $x, y$ location of a Component.

2. The part number of a Component.

3. Association of the placed Component to the defined Component.

4. Component which must be inserted manually.

5. Component on the bottom side of the LEP PWB.

6. Component on the top side of the LEP PWB.

Test Group 15: Panel; PWB.

Test purposes: 
1. For the next graphical display.

2. Color, linefont, display thickness.

3.A symbol for each different drill size [reference to MIL-D-28000 Class IV].

Test Group 16: Production Engineering (process sheets).

Test purposes:

1. Identify components, component outlines, product outline.

2. Appearance (see IGES Drafting AP).

Test Group 17: Technical Illustrations.

1. Appearance (see IGES Drafting AP).

Test Group 18: Dimensions.

Test purposes:

1. Dimensions, views (see IGES Drafting AP).

2. Appearance (see IGES Drafting AP).

3. Font, color, DE Line weight.

\subsection{LEP Constructs}

The layered electrical product is a complex assembly made up of both graphic and non-graphic information, interrelated through parent/child relations and associations.

\subsubsection{LEP Constructs Supporting Signal Conductivity}

Traces: Traces (JOIN objects)use simple geometry entities such as lines and arcs. They interconnect electronic Components and carry Signals. Physically, they have a specific width. Portions of the resulting Trace may be deposited on more than one layer. Traces usually pass between the different layers by means of VIAS and through-pins.

Jumpers: Jumpers are a physical interconnect which may be implemented as a component or a trace. The jumper and the other JOINS it connects shall bear a single signal name.

Planes: Planes, such as ground and power planes, outline the appropriate (filled) areas.

Vias: VIAS are holes made between two or more layers on the LEP. They are plated so they can carry the Signal. The holes are made by different means such as drilling, milling or cutting. This variability makes it hard to select specific entities which relate to some aspects of Vias. However one makes the hole, the functions of the Via are still the same. Vias may be through, blind, or buried. Vias are often used as Test Points.

Padstacks (through and SMT): Padstacks provide the electrical interface between the LEP PWB and the Component. Padstacks may be either through-hole or surface mount. Each Padstack shall have a LEP defined property to identify which type it is. 


\subsubsection{LEP Constructs Supporting a Physical Representation}

A physical LEP shall contain at least one simple closed planar geometry entity depicting the LEP outline. This LEP CLOSED CURVE may be a KEEPIN/KEEPOUT, tool path, or any other functional type of closed geometry entity.

Several applications require a PDQ LEP representation. Computer controlled equipment may then extract needed data without manual intervention. For example, Component insertion equipment can put a Component at the X, Y coordinates specified by the Network Subfigure Instance Entity (Type 420) locating the Component.

Operations which modify the physical representation of the LEP, such as scaling or rotation, will have adverse affects on some application systems. Constructs which are beyond the scope of an automated operation (such as dimensions and view/draw) could stall or disable the processing system.

\subsubsection{LEP Constructs Supporting a Logical Representation}

A logical LEP element (e.g., a gate or a driver) shall be represented by a Network Subfigure Definition Entity (Type 320). The Definition may or may not reference a CLOSED CURVE depicting a schematic symbol. In either case, the Network Subfigure Definition Entity (Type 320) shall list the ports corresponding to physical connect points. The corresponding Connect Points (Type 132) referenced by the Network Subfigure Instance Entity (Type 420) may participate in logical flow associativities. Physical locations are not needed. If present, they locate symbols and port representations in the "drawing space" of the schematic.

\subsection{Entity Usage in a PDQ LEP Representation}

IGES allows many different ways to add semantics to a file. Among these ways are using properties, associativities, groupings, and even attributes. One way uses the level on which an entity is found. Another uses the color of the entity. These may seem like clever schemes which make it easy for users or vendors to support the semantic convention. However, it actually leads to confusion, frustration, and reduced semantic information exchange when the convention is not both well documented and universally supported.

\section{The Application Protocol for a PDQ LEP will focus on the use of IGES Property Entities} (Type 406) to add more semantic information to the LEP.

This section specifies what entities may appear in conformant files in a PDQ LEP representation. This section also specifies what entities are required in a PDQ LEP. Some of the required entities are Property Entities (type 406). It is not difficult for a pre-processor to include these Properties when writing an IGES file. This approach does assume though, that the data for the Properties do exist in the native database of the sending system. For example, the component_height property is required for several applications. However, if the value is not contained in the part data within the originating CAD system, the component_height property cannot be generated in a conformant way. This may lead to rejection of an IGES file for non-conformance when the fault was not with the IGES preprocessor.

Please remember that all rules and requirements which apply to all IGES entities used still apply to those conformant to the LEP AP. For example, a zero length text string is non-conformant and the maximum pre-defined Color Number is eight. 


\subsubsection{DE Fields are NOT Expected to Convey Meaning}

DE fields shall not convey any semantic information beyond their specific use.

DE fields $1,2,10,11,14,15$ and 20 are artifacts of the physical format of the IGES file, and shall not provide semantic information.

Table 6-1. Overview of DE Settings for PDQ

\begin{tabular}{|l|l|l|l|}
\hline Note & \multicolumn{1}{|c|}{ DE Field Name } & $\begin{array}{c}\text { DE } \\
\#\end{array}$ & Acceptable Values \\
\hline \hline a & Structure & 3 & 0 \\
\hline b & Line Font & 4 & 1 \\
\hline c & Level & 5 & 0 to 1024 \\
\hline d & View & 6 & 0 \\
\hline e & Transformation Matrix & 7 & \#, or pointer \\
\hline f & Status; Blanked & 9.1 & *ee \\
\hline g & Status; Subordinate & 9.2 & *ee \\
\hline h & Status; Entity Use & 9.3 & *ee \\
\hline i & Status; Hierarchy & 9.4 & *ee \\
\hline j & Line Weight & 12 & per IGES \\
\hline k & Color & 13 & 0 to 8 \\
\hline l & Reserved (blank) & 16 & “" \\
\hline l & Reserved (blank) & 17 & “" \\
\hline m & Label & 18 & “" " \\
\hline m & Entity Subscript & 19 & 0 or “" \\
\hline n & Label Display & 8 & 0 or “" \\
\hline
\end{tabular}

(a) Structure (DE 3): DE field 3 has no relevance to any portion of the LEP AP and shall be set to zero.

(b) Line Font (DE 4) No functionality shall be provided through Line Font. Line Font shall be 1 for all entities of a PDQ LEP.

(c) Levels (DE 5): It is a common practice in the design of a layered electrical product to use the system's "layers" to specify functionality. For example, LEP signal Traces may be assigned layers 1 through 24; layer 31 has top surface reference designators, and so on. While the practice is common, the layering convention is not. In fact, there is often a lack of consistency in layering conventions within the same design group. Thus, the layer a particular entity is on, shall convey no functionality in and of itself. Yet, layering must be understood and processed.

Therefore, a Level Function Property (type 406 form 3) shall be applied to each level. The Level Func- 
tion stands alone, the level it applies to need not be in the file.

Please refer to Appendix [A?] for a list of function names and descriptions.

(d) Views (DE 6) The value shall be zero for all entities which are part of the PDQ PWB.

(e) Transformation Matrix (DE 7)

(f) Status; Blanked (DE 9.1)

(g) Status; Subord (DE 9.2)

(h) Status; Entity Use (DE 9.3)

(i) Status; Hierarchy (DE 9.4)

$(* * e)$ These switches vary, refer to specific entity for correct settings.

(j) Line Weight (DE 12) Line Weight shall imply no functionality. Also, the Line Widening Property (type 406 form 5) shall represent Trace thickness.

(k) Color (DE 13): Color shall imply no functionality. One of the eight non-zero Color Numbers shall appear in the proper DE field 13 for every entity having a color attribute. The Color Number may appear in field 13 of the entity itself, or in field 13 of the Color Definition (type 314) Entity referenced. See IGES section 4.72 for additional requirements.

(1) DE fields 16 and 17 have no relevance to any portion of the LEP AP and shall ignored. Both fields shall contain blank characters only.

(m) DE field 18 and 19 taken together uniquely identify the entity within the range of the IGES file. The Entity Label may provide a name to distinguish each particular kind of usage of a given Entity Type from others having the same Entity Type Number and Form number. This AP does not require usage of this construct.

(n) Label Display (DE 8) This field shall contain either a zero or blanks.

\subsubsection{Entities Which MAY Appear in a PDQ LEP Representation}

\subsubsection{LEP Usage of Geometry}

Geometry entities may represent many distinct kinds of LEP objects and features. Examples include a LEP outline, a surface mount Pad, and a strangely shaped power plane. In each case, the geometric shape depicts the object or feature graphically. In some cases, the same IGES entity types may have been used for each object. Thus, no semantic information is provided by most Geometry (type $1 \mathrm{xx}$ ) entities.

Geometry entities may also represent non-electrical objects and features such as tooling holes, FIDUCIALS, LEP outline(s), and heat sinks. Electrical parts such as Vias, Components, edge connectors, paste resistors, SMT Pads, testpoints, Padstacks, Traces, jumpers, and signal planes are represented in like manner.

Most of the geometry (but not all) will be on the X-Y plane, with $\mathrm{Z}$ being 0.0 . Even the geometry representing signals on top, inner, and bottom layers will have $\mathrm{z}=0.0$. The $Z$ depth of each layer is determined by the substrate thickness and the sequence of layer assembly.

The bulk of the LEP Application needs for geometry do not require exotic Geometry entities. So, a reduced set of Geometry entities permitted in an LEP AP conformant file has been defined. This reduced set, the "LEP Geometry Entities," is defined below.

There are two uses of geometry in the LEP AP. One use is to define enclosed areas, the other is to define a non-closed linear path.

Enclosed areas may be defined with a single circle, a multi-segment line, or a combination of 
geometries which are grouped together. A single Geometry Entity shall represent or associate the constituent Geometry Entities that enclose the given area. The Circular Arc Entity (Type 100) and Copious Data Enclosed Planar Curve Entity (Type 106, Form 63) are examples of single entity enclosed areas. Multi-entity paths which form an enclosed area and are subordinate to an occurrence of a Composite Curve Entity (Type 102) typify a multi-entity enclosed area.

Levels may imply grouping, but shall not imply function. The Level Function Property Entity: (Type 406, Form 3) shall assign function.

The following "LEP Geometry Entities" (as restricted in this section) may appear in LEP AP conformant files:

\begin{tabular}{ll} 
Circular Arc Entity & (Type 100) \\
Copious Data Entity & (Type 106, Forms 11,12,13, and 63) \\
Line Entity & (Type 110) \\
Point Entity & (Type 116) \\
Flash Entity & (Type 125) \\
Connect Point Entity & (Type 132) \\
Composite Curve Entity & (Type 102) \\
Plane Entity & (Type 108) \\
Transformation Matrix Entity (Type 124) \\
\multicolumn{2}{c}{ (The determinant of the Transformation Matrix shall be +/-1.0) } \\
(no other scaling shall appear in conformant files.))
\end{tabular}

Filled areas shall use the Sectioned Area Entity (Type 230). Only the (full circle) Circular Arc Entity (Type 100), Composite Curve Entity (Type 102), Flash Entity (Type 125) and Simple Closed Planar Curve Entity (Type 106, Form 63) shall represent areas which are shown filled. The Flash Entity (Type 125) shall not reference a Transformation Matrix.

\subsubsection{LEP Usage of Annotation}

The following annotation entities may appear in conformant files:

General Note Entity (Type 212, Form 0)

Sectioned Area (fill area) (Type 230 fill patterns 0,19 only)

The Sectioned Area Entity (Type 230) shall depict filled areas such as irregularly shaped signal planes, ground planes or power planes. Only the (full) Circular Arc Entity (Type 100), Composite Curve Entity (Type 102), and Simple Closed Planar Curve Entity (Type 106, Form 63) shall be filled by the Sectioned Area Entity. Only the unfilled Fill Pattern (0) and the solid filled Fill Pattern (19) of the Sectioned Area Entity (Type 230) shall appear in conformant files.

No other annotation entities shall appear in conformant files. 
Table 6-2. DE Settings of Annotation Entities in a PDQ IGES File

\begin{tabular}{|ll|l|l|}
\hline \multicolumn{1}{|c|}{ Annotation Entity - DE Field } & \multicolumn{1}{c|}{$\begin{array}{c}\text { General } \\
\text { Note }\end{array}$} & $\begin{array}{c}\text { Sectioned } \\
\text { Area }\end{array}$ \\
\hline \hline Line Font & $(4)$ & 0,1 & 0,1 \\
\hline Level & $(5)$ & 0 to 1024 & 0 to 1024 \\
\hline View & $(6)$ & 0 & 0 \\
\hline Transformation Matrix & $(7)$ & 0, pointer & 0, pointer \\
\hline Status; Blanked & $(9.1)$ & 00 & 00 \\
\hline Status; Subordinate & $(9.2)$ & 01 & 01 \\
\hline Status; Entity Use & $(9.3)$ & 01 & 01 \\
\hline Status; Hierarchy & $(9.4)$ & 01 & 01 \\
\hline Line Weight & $(12)$ & per IGES & per IGES \\
\hline Color & $(13)$ & 0 to 8 & 0 to 8 \\
\hline
\end{tabular}

The following entities may appear in conformant files:

Subfigure Definition Entity $\quad$ (Type 308)

Subfigure Instance Entity (Type 408)

Network Subfigure Definition Entity (Type 320)

Network Subfigure Instance Entity (Type 420)

Text Display Template Entity (Type 312)

Color Definition Entity (Type 314)

Support of this entity (the Type 314 ) is optional for both pre- and post- processors. Please refer to the discussion in section 6.3.1.

\subsubsection{LEP Usage of Existing Specific Property Entities}

One of the easiest and most widely supported methods for adding more semantic information to an entity or group of entities is the Property Entity (Type 406). A property is a well defined mechanism in IGES. One entity may have several Properties. One Property may apply to many entities. An entity may exist with no Properties, and a Property may be in a file but not referenced by any entity. The second group of additional pointers (see IGES section 2.2.4.4.2) provide the mechanism for referencing Properties.

The conformant LEP representation will use Properties in adding both graphic and non-graphic information in the file. Properties are of Type 406. The conformant LEP representation will use existing Properties whenever possible. The Generic Data Properties detailed in this AP shall be used in most other cases. This means that other methods of adding semantic information (such as 
the Attribute Table) will not be conformant.

Definition Level Property Entity (Type 406, Form 1) allows an entity (or entities) to exist on more than one level. The DE Level field of the target entity (or entities) contains a pointer to the Definition Levels Property. The Definition Levels Property will list all the levels on which the target entities exist. For example, the DE field of a Drilled Hole Property may contain a pointer to the Definition Levels Property which lists all the levels penetrated by the drilled hole.

Region Restriction Property Entity (Type 406, Form 2) may identify keepin/keepout areas for Traces, Components and Vias.

Level Function Property Entity (Type 406, Form 3) may describe intent of specific level.

Line Widening Property Entity (Type 406, Form 5) may specify line geometry thickness (e.g. to depict Trace width).

Drilled Hole Property Entity (Type 406, Form 6) may describe a drilled hole size, plating, and affected levels.

Part Number Property Entity (Type 406, Form 9) assigns up to four different unique identifiers for referencing a Component. The property provides for a generic part number, a military standard part number, a vendor part number, and an internal part number. Components (such as those represented by a Network Subfigure Instance) are the primary users of this Property.

Name Property Entity (Type 406, Form 15). The name Property may exist in the file, but shall not convey LEP semantic information. Specifically, it may name a level, but it shall NOT imply a level function.

\subsubsection{LEP Usage of the (Augmented) Generic Data Property Entity}

Several new Properties are introduced in order to simplify and extend the capability of IGES for the exchange of LEP semantic information. These Properties will all share a common form number. Thus, they must use pattern matching to determine the name, and therefore the function, of the Property. While this method increases processing time, it does allow a file to be complete and self contained. See Appendix ?? for the list of these new Generic Properties.

\subsubsection{Entities Which Shall NOT be Used in a PDQ LEP Representation}

The following entities are those which have similar functionality to recommended entities. They shall not be used in LEP AP conformant files.

Attribute Table Definition Entity (Type 322)

Attribute Table Instance Entity (Type 422)

While the Attribute Table Definition does have many attributes already defined, some implementors may think it too onerous.

Reference designator Property Entity (Type 406, Form 7)

Reference designator is assigned in Network Subfigure Instance (type 420)

Pin Number Property Entity $\quad$ (Type 406, Form 8)

Pin number is assigned in Connect Point Entity (Type 132) 


\subsection{LEP Entity Usage for Core Applications}

Some physical representations are common to many different application areas. This section will discuss them.

The LEP application includes engineering and design tasks such as schematic entry, netlist generation, auto-placement, auto-route, and Component engineering.

This LEP application DOES require a PDQ data repository.

All constructs specified in 6.5.1 apply when an LEP product is fully contained in a single file. When the LEP is described with a hierarchy of designs - each design in a separate file-the PWB component of the assembly will contain those features required for the PWB only as described in 6.5.4. When the hierarchy of designs is used, features such as padstacks, traces, drilled holes, and fiducials are found in the PWB file, and the LEP assembly file includes the identification of the PWB design required. NOTE: The use of "PWB" is intended to include the substrate for a MCM or other LEP product-types.

\subsubsection{LEP Entity Usage for Padstack Representation}

A Padstack shall be represented by an ordinary Subfigure Definition Entity (Type 308) as specified in 5.3.2.17.

The Padstack shall contain, at a minimum, one simple Closed Curve which represents a conductive area. That conductive area joins two signal carriers into one. This conductive area shall exist on at least one Level in the IGES file. That level shall be set in the Level field of the geometry entity. [The desired setting may require the use of a Hierarchy Property Entity (Type 406, Form 10).] More than one layer may share the same conductive area by application of the Definition Levels Property Entity (Type 406, Form 1).

Different geometric shapes which perform different functions (such as isolating a through-pin from a signal plane) may appear in the Padstack.

\subsubsection{LEP Entity Usage for Physical Padstack Definition}

\section{Entities required:}

A Subfigure Definition Entity (Type 308) shall define each Physical Padstack. Each such definition shall have a Generic Property. Entity.(Type 406, Form 27) named component_default_padstack.

A CLOSED CURVE shall enclose the area of each Land in the Physical Padstack. See Geometry below for more details.

A Sectioned Area Entity (Type 230) shall fill the CLOSED CURVE which depicts a LAND. Only the Fill Patterns specified by code 0 (blank fill) and code 19 (solid fill) of the Sectioned Area Entity (Type 230) shall appear in conformant files.

\section{Geometry -}

At least one CLOSED CURVE shall define the outline of the area which represents the conductive Land in a Padstack. One of four LEP Geometry Entities may depict each Land. Those four are: the (full) Circular Arc Entity (Type 100), the Composite Curve Entity (Type 102), the Flash Entity (Type 125), or the Simple Closed Planar Curve Entity (Type 106, Form 63). 


\section{Annotation -}

The CLOSED CURVES(s) enclosing the conductive area of the Land(s) in the Physical Padstack shall (each) reference a Sectioned Area Entity (Type 230). That Sectioned Area shall reference a Generic Property Entity (Type 406, Form 27) named component_pkg_type (=pad). No other Annotation Entities shall appear as part of a Physical Padstack representation.

\section{Structure -}

The Subfigure Definition Entity (Type 308) shall depict the Physical Padstack. The Transformation Matrix field of the Subfigure Definition shall be zero. The Subfigure Definition shall reference each CLOSED CURVE described above as annotation. Each Closed Curve shall reference a Sectioned Area Entity (Type 230) with a Fill Pattern of 0 or 19.

\section{Associativities -}

Conformance to the LEP AP does not require any Associativities in the definition of the Physical Padstack.

\section{Properties -}

The Subfigure Definition Entity (Type 308) defining the Padstack shall reference the following properties:

Generic Property Entity (Type 406, Form 27) named physical_padstack_definition

Generic Property Entity (Type 406, Form 27) named component_pkg_type (=pad).

\subsection{Entities Required to Add the Representation of Drilled Holes into a Padstack.}

Entities required:

A Point Entity (Type 116) shall locate the Hole. Each such Point Entity shall reference a Drilled Hole (Type 406, Form 6) Property.

Geometry -

A Point Entity shall provide the $x, y$ location of the Hole(s) within the Padstack definition space.

Annotation -

Conformance to the LEP AP does not require any Annotation Entities in the representation of the Padstack.

\section{Structure -}

The Padstack Subfigure (type 308) shall reference one Point Entity (Type 116) for each hole. The Transformation Matrix field of the Point Entity (type 116) shall be 0.0. The Point Entity (Type 116) shall reference the Drilled Hole Property (Type 406, Form 6).

If the Point Entity (Type 116) references another Subfigure Definition Entity (Type 308) for display purposes, that definition shall exist in the same physical IGES file as the Point. More than one Padstack may use the same display definition.

\section{Associativities -}

Conformance to the LEP AP does not require any Associativities in the representation of the Point. 


\section{Properties -}

The Point Entity (Type 132) locating the Padstack Hole shall reference the following Property:

The Drilled Hole (Type 406, Form 6) Property.

The Drilled Hole Property shall specify the Drill diameter size, the Finish diameter size, and the Plating indication flag of a plated Component Pin or Via hole. The DE Level field of the Drilled Hole Property Entity (Type 406, Form 6) shall be zero for a through-hole which passes through the entire LEP. The DE Level field of the Drilled Hole Property Entity (Type 406, Form 6) shall reference a Definition Levels Property Entity (Type 406, Form 1) for a Hole which does NOT go all the way through the PWB. That Definition Levels Property shall indicate which levels are penetrated.

The Point Entity (Type 132) locating the Padstack Hole may reference either or neither of the following two Properties:

The test_point Property Entity (type 406, Form 27) named not_allowable_test_point or the test_point Property Entity (Type 406, Form 27) named allowable_test_point.

If neither is referenced, the not_allowable_test_point semantic applies.

\subsection{Entities Required to Add Display Appearance of Holes in a Padstack.} Entities required:

A Point Entity (Type 116) locating the Padstack Hole may reference a Subfigure Definition Entity (type 308) to display the presence of the HOLE. If the Point does not reference a Subfigure Definition in the file, the receiving system may display the default system icon for Point graphics instead.

\section{Geometry -}

A Subfigure Definition Entity (Type 308) may depict the presence of the HOLE as illustrated in Figure 6-2. One CLOSED CURVE may define the outline of the area which represents the HOLE in a PADSTACK.

\section{Annotation -}

Conformance to the LEP AP does not require any Annotation Entities in the representation of the HOLE.

\section{Structure -}

The Point Entity (Type 116) may reference one Subfigure Definition (Type 308). The Transformation Matrix field of the Point Entity (Type 116) shall be zero. 
Table 6-3. IGES Data Fragments Showing Padstack Structures

\begin{tabular}{|c|c|l|l|}
\hline Entity Type & Form & DE Status & \multicolumn{1}{|c|}{ Parameter Data } \\
\hline \hline 308 & 0 & 010201 & $308,0,11$ Hpad_060_038,3,3,5,7,0,1,9; \\
\hline 100 & 0 & 010001 & $100,0.0,0.0,0.0,0.09,0.0,0.03,0.0 ;$ \\
\hline 230 & 0 & 010101 & $230,03,19,0.0,0.0,0.0,0.005 ;$ \\
\hline 116 & 0 & 010001 & $116,0.0,0.0,0.0,0,1,11 ;$ \\
\hline 406 & 27 & 020301 & $406,2,3,8$ HPadstack,3,8HThru_Pad; \\
\hline 406 & 6 & 020301 & $406,5,0.046,0.038,1 ;$ \\
\hline 408 & 0 & 000001 & $408,1,0.2,0.2,0.0,1.0 ;$ \\
\hline
\end{tabular}

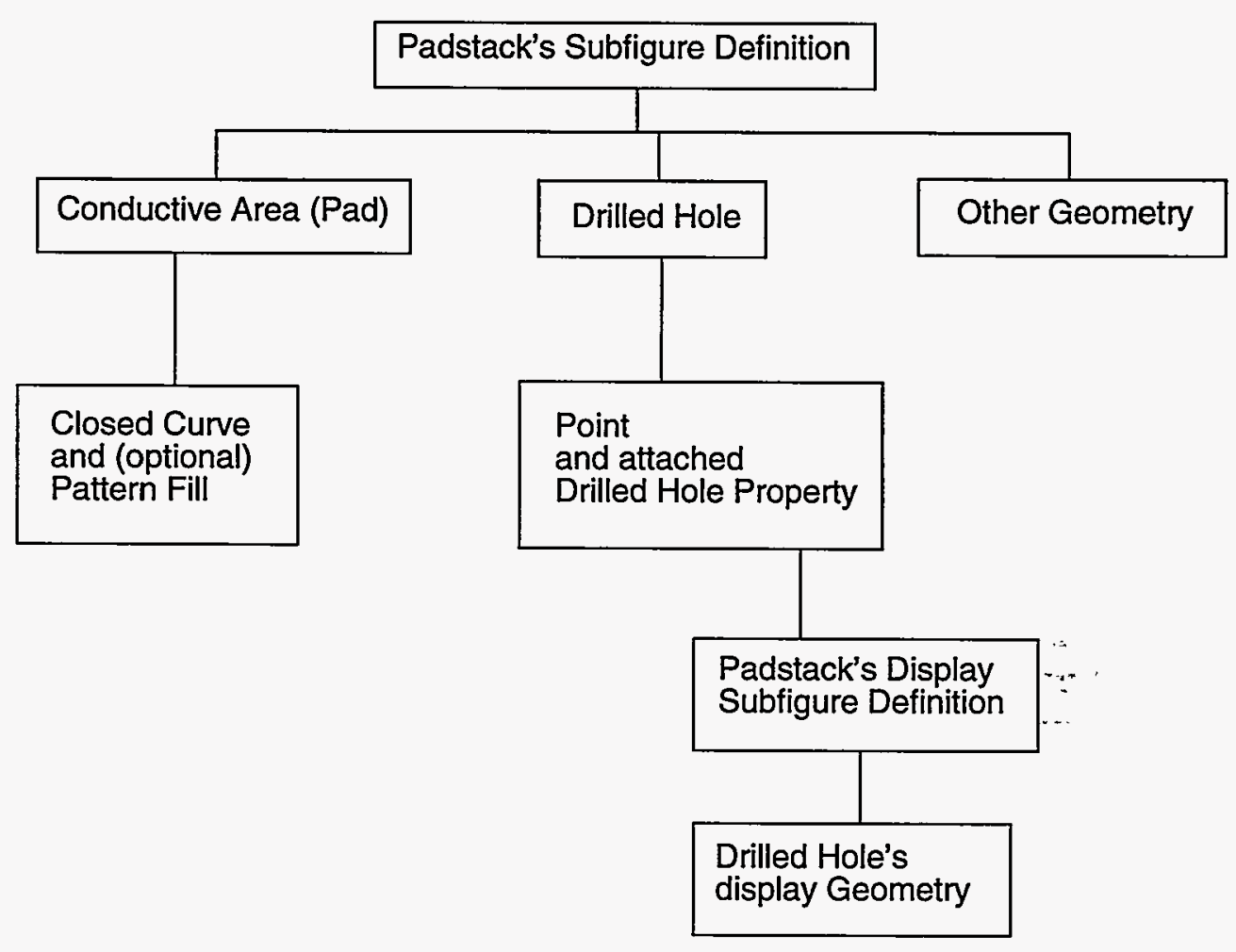

Figure 6-2. General Structure of Objects (See Section 5.2) Found in a PADSTACK Associativities -

Conformance to the LEP AP does not require any Associativities in the representation of the Point 
Entity (Type 116).

Properties -

The Subfigure Definition Entity (Type 308) depicting the Padstack Hole shall reference the following Property:

Generic Property Entity (Type 406, Form 27) named physical_outline.

\subsubsection{LEP Entity Usage for Physical Component Representation}

There are two parts to the representation of Physical Components in an IGES file. The first part is the definition. The second part is the instance. Multiple occurrences of similar Physical Components may share a single Definition. Connect Points in each Instance need to correspond exactly to their counterparts in the Definition. Circuit nodes, or Signals are represented by Flow Associativity Entities (Type 402, Form 18).

\subsubsection{LEP Entity Usage for Physical Component Definition}

The Physical Component itself is simple to represent. It requires a Network Subfigure Instance Entity (Type 420). Although it may be shared with other Instances, a Network Subfigure Definition Entity (Type 320) is also needed. One Connect Point Entity (Type 132) for each PIN or Pad is also needed. The Connect Points of the Instance may each reference a Flow Associativity Entity (Type 402, Form 18). The Flow Associativity groups all the pertinent objects subjected to the same electrical signal (NET).

Entities required:

A Network Subfigure Definition Entity (Type 320) shall define each Physical Component in the AP conformant files. Each Network Subfigure Definition (Type 320) shall reference as many LEP Geometry Entities as needed to depict the Component. Each Network Subfigure Definition Entity (Type 320) may reference many of the properties listed below. Conformance to the LEP AP does not require any Text Display Template Entities (Type 312) in the representation of the Physical Component. At least one Connect Point Entity (Type 320) shall provide a means of connecting signals to Pin 1 of the Component.

\section{Geometry -}

One Closed Curve shall define the outline of the Physical Component in the X, Y plane of the LEP. Each Closed Curve shall have one LEP Property attached to it which defines its function. Examples of LEP functions include: placement_outline, thermal_outline, physical_outline, and footprint. Please refer to Appendix [A?] for a complete list.

Any LEP Geometry Entity may appear as part of the Component Geometry. All LEP Geometry shall lie entirely in the $\mathrm{Z}=0.0$ plane.

\section{Annotation -}

Conformance to the LEP AP does not require any Annotation Entities in the representation of the Physical Component Definition.

\section{Structure -}

The Network Subfigure Definition Entity (Type 320) shall reference one or more LEP Geometry Entities. Each Geometry Entity may reference some properties. The Network Subfigure Defini- 
Implementation \& Conformance Testing

tion Entity (Type 320) shall reference at least one property. The Network Subfigure Definition Entity (Type 320) Type Flag shall indicate $(\mathrm{TF}=2)$ because the type is physical. The Network Subfigure Definition Entity (Type 320) shall reference one (for pin 1) or more Connect Point (Type 132) entities. The Transformation Matrix field of the Network Subfigure Definition Entity (Type 320) shall be zero.

Associativities -

Conformance to the LEP AP does not require any Associativities in the representation of the Component Definition.

Properties -

Mandatory Properties:

The Network Subfigure Definition Entity (Type 320) defining the Physical Component shall reference the following properties:

The Generic Property Entity (Type 406, Form 27) named component_std_name.

The Generic Property Entity (Type 406, Form 27) named component_version.

Optional Properties:

The Physical Component Definition (type 320) may reference the following properties:

The Generic Property Entity (Type 406, Form 27) named component_pkg_type.

The Generic Property Entity (Type 406, Form 27) named component_tech_type.

The Generic Property Entity (Type 406, Form 27) named component_layout_surface.

The Generic Property Entity (Type 406, Form 27) named component_insertion_origin.

The Generic Property Entity (Type 406, Form 27) named component_insertable.

The Generic Property Entity (Type 406, Form 27) named component_height.

The Generic Property Entity (Type 406, Form 27) named component_max_size.

The Generic Property Entity (Type 406, Form 27) named component_outline_overhang.

\subsubsection{LEP Entity Usage for Physical Component Instance}

Entities required:

The Network Subfigure Instance Entity (Type 420) locates the Component occurrence and provides a way to connect to its ports. A Connect Point Entity (Type 132) models each port (Pin or $\mathrm{Pad})$. The Reference Designator shall be provided in the PRD parameter of the Network Subfigure Instance Entity (Type 420). The Part Number(s) shall be provided in the Part Number Property Entity (Type 406, Form 9). Other properties provide such information as whether the Component is attached to the top or the bottom of the LEP and how many 90 degree rotations perpendicular to the surface of the LEP are required of insertion processes.

\section{Geometry -}

A Connect Point Entity (Type 132) having a Generic Property Entity (Type 406, Form 27) named pad_tech_type shall represent each Component Pin or each Component Pad in LEP AP conformant files. That property shall specify whether the Pad or Pin is of type surface mount (SMT) or 
through-hole. One of the Connect Points shall also reference a Generic Property Entity (Type 406, Form 27) named pin1.

One Connect Point Entity (Type 132) shall appear in the Network Subfigure Instance Entity (Type 420) for each Pin in the referenced Network Subfigure Definition, whether the Pin is actually used or not.

\section{Annotation -}

Conformance to the LEP AP does not require any Annotation Entities in the representation of the Physical Component Instance.

\section{Structure -}

The Network Subfigure Instance Entity (Type 420) shall reference one and only one Network Subfigure Definition Entity (Type 320). The Instance shall also specify the location of the Component with respect to the origin of the LEP.

The Network Subfigure Instance Entity (Type 420) shall reference one or more Connect Point Entities (Type 132).

Associativities -

The Connect Points of the Instance may each reference a Flow Associativity Entity (Type 402, Form 18). The Flow Associativity groups all the pertinent objects subjected to the same electrical signal.

Properties -

Mandatory Properties:

The Network Subfigure Instance Entity (Type 420) modeling the Component shall reference the following properties:

Part Number Property Entity (Type 406, Form 9)

Generic Property Entity (Type 406, Form 27) named component_instance_side

Generic Property Entity (Type 406, Form 27) named component_instance_rotation.

Each Connect Point Entity (Type 132) modeling a Component Pad or Pin shall reference the following LEP property:

The Generic Property Entity (Type 406, Form 27) named pad_tech_type. Furthermore, the Connect Point Entity Entity (Type 132) modeling the Component Pad or Pin bearing the "pin1" designation shall reference the following LEP property:

The Generic Property Entity (Type 406, Form 27) named pin1

Optional Properties:

The Network Subfigure Instance Entity (Type 420) may reference any of the following seven LEP Generic Property Entities (Type 406, Form 27):

component_preplace

abs_voltage_max

tolerance

therm_cond

therm_jc 
therm_r

junction_max_t

\subsubsection{LEP Entity Usage for Physical Connect Point}

Entities required:

Connect Point Entities (Type 132) shall locate the place where a connection may occur between the Component and the LEP. One Connect Point Entity (Type 132) shall appear in the Network Subfigure Definition for each Pin in the corresponding Physical Component.

\section{Geometry -}

See Entities required above and Structure below. Any LEP Geometry Entity may appear as part of the Physical Connect Point geometry. All LEP Geometry shall lie entirely in the $\mathrm{Z}=0.0$ plane.

\section{Annotation -}

Conformance to the LEP AP does not require any Annotation Entities in the representation of the Physical Connect Point.

\section{Structure -}

The Connect Point Entity (Type 132) may reference one or more LEP Geometry Entities.

Each Geometry Entity may reference some properties. The CID parameter of the Connect Point shall provide the Pin Number. The Transformation Matrix field of the Connect Point shall be 0.0.

\section{Associativities -}

Each Connect Point Entity may reference a Flow Associativity Entity (Type 402, Form 18). The Flow Associativity groups all the pertinent objects subjected to the same electrical signal.

\section{Properties -}

Each Connect Point Entity modeling a Physical Connect Point may reference the following Properties:

Definition Levels (Type 406, Form 1) Property.

The Generic Property (Type 406, Form 27) named pad_tech_type (smt/through-hole).

The Generic Property (Type 406, Form 27) named pin1.

\subsubsection{LEP Entity Usage for Physical Via}

\section{Entities required:}

The Connect Point Entity (Type 132) models the Via. The Singular Subfigure Instance Entity (Type 408) may depict the shape of the Via. The Connect Point Entity may reference at most one Singular Subfigure Instance Entity in LEP AP conformant files.

\section{Geometry -}

The Connect Point Entity (Type 132) locates the Via within the LEP. A Singular Subfigure Instance Entity (Type 408) having a Generic Property Entity (Type 406, Form 27) named standalone_via may tag each Via. Each such Subfigure Instance may reference any LEP Geometry Entity. 


\section{Annotation -}

Conformance to the LEP AP does not require any Annotation Entities in the depiction of the Via. Structure -

The Connect Point Entity (Type 132) modeling a Via shall not reference an owning entity.

The Connect Point Entity (Type 132) modeling a Via may reference one Singular Subfigure Instance Entity (Type 408) to graphically depict the presence of a Via at the indicated location.

If the Connect Point Entity (Type 132) does not reference a Subfigure Definition in the LEP AP conformant file, the receiving system may display its default icon for Via graphics.

\section{Associativities -}

The Connect Point Entity Entity (Type 132) modeling a Via shall reference the proper Flow Associativity Entity (Type 402, Form 18) to connect the Via to the signal join.

\section{Properties -}

Mandatory Properties:

The Connect Point Entity (Type 132) modeling a Via shall reference the following LEP properties:

The Generic Property Entity (Type 406, Form 27) named stand-alone_via.

Either the Generic Property (Type 406, Form 27) named allowable_test_point or the Generic Property Entity (Type 406, Form 27) named not_allowable_test_point.

Drilled Hole (Type 406, Form 6) Property.

The PWB Drilled Hole Property shall specify the Drill diameter size, the finish diameter size, and the Function Code (5) of a plated Via HOLE. The DE Level field of the Drilled Hole Property shall be zero for a through-hole which passes through the entire LEP. The DE Level field of the PWB Drilled Hole Property shall reference a Definition Levels Property Entity (Type 406, Form 1) for a HOLE which does NOT go all the way through the PWB. That Definition Levels Property (Type 406, Form 1) shall specify which levels are penetrated.

\section{Optional Property:}

Each Connect Point Entity (Type 132) modeling a Via may reference a Definition Levels Property Entity (Type 406, Form 1).

Table 6-4. DE Settings of Via Entities in a PDQ IGES File

\begin{tabular}{|c|c|c|c|c|}
\hline \multicolumn{2}{|c|}{ Component Entity - DE Field } & $\begin{array}{l}\text { Network } \\
\text { Subfigure }\end{array}$ & $\begin{array}{l}\text { Network } \\
\text { Subfigure }\end{array}$ & $\begin{array}{c}\text { Connect } \\
\text { Point }\end{array}$ \\
\hline Line Font & (4) & 0,1 & 0,1 & 0,1 \\
\hline Level & (5) & 0 to 1024 & 0 to 1024 & 0 to 1024 \\
\hline View & (6) & 0 & 0 & 0 \\
\hline
\end{tabular}


Implementation \& Conformance Testing

Table 6-4. DE Settings of Via Entities in a PDQ IGES File

\begin{tabular}{|ll|l|l|l|}
\hline Component Entity - DE Field & $\begin{array}{c}\text { Network } \\
\text { Subfigure } \\
\text { Definition }\end{array}$ & $\begin{array}{c}\text { Network } \\
\text { Subfigure } \\
\text { Instance }\end{array}$ & $\begin{array}{c}\text { Connect } \\
\text { Point }\end{array}$ \\
\hline \hline Transformation Matrix & $(7)$ & 0 & 0 or pointer & 0 or pointer \\
\hline Status; Blanked & $(9.1)$ & 00 & 00 & 00 \\
\hline Status; Subordinate & $(9.2)$ & 01 & 01 & 01 \\
\hline Status; Entity Use & $(9.3)$ & 02 & 00 & 00 \\
\hline Status; Hierarchy & $(9.4)$ & 01 & 01 & 01 \\
\hline Line Weight & $(12)$ & per IGES & per IGES & per IGES \\
\hline Color & $(13)$ & 0 to 8 & 0 to 8 & 0 to 8 \\
\hline
\end{tabular}

Note 1: The preferred method of displaying text found in the $320,420,132$, and 408 is by using the text display template. However, a preprocessor may display text using the General Note Entity (Type 212) instead. The only concern with using this method is that it is possible to get the PDQ intelligence and the General Note Entity (Type 212) text out of agreement.

\subsubsection{LEP Entity Usage for Physical Mechanical Part Representation}

Entities required:

The Singular Subfigure Instance and Subfigure Definition Entities model the Mechanical Part in LEP AP conformant files. The Singular Subfigure Instance Entity (Type 408) shall locate and depict the Mechanical Part. It shall reference one Subfigure Definition (which may also be referenced by other Instances.) The Subfigure Definition Entity (Type 308) shall reference one LEP Closed Curve depicting the X, Y outline of the Mechanical Part. The LEP Closed Curve may reference one or more other LEP Geometry Entities. Various properties shall add the needed semantics.

Geometry -

A Closed Curve having a Generic Property Entity (Type 406, Form 27) “physical_outline" Property shall depict each Mechanical Part.

Annotation -

Conformance to the LEP AP does not require any Annotation Entities in the representation of the Mechanical Part.

Structure -

See the discussion under Required Entities above.

Associativities -

Conformance to the LEP AP does not require any Associativities in the representation of the Mechanical Part. 


\section{Properties -}

\section{Mandatory Properties:}

The Subfigure Definition Entity (Type 308) depicting the Mechanical Part shall reference the following property:

The Generic Property Entity (Type 406, Form 27) named physical_outline.

The Singular Subfigure Instance Entity (Type 408) locating the Mechanical Part shall reference the following three properties:

Part Number Property Entity (Type 406, Form 9),

Generic Property Entity (Type 406, Form 27) named component_instance_side, and

Generic Property Entity (Type 406, Form 27) named component_instance_rotation.

Optional Properties:

The Subfigure Definition Entity (Type 308) depicting the Mechanical Part may reference the following properties:

The Generic Property Entity (Type 406, Form 27) named component_std_name.

The Generic Property Entity (Type 406, Form 27) named component_version.

The Generic Property Entity (Type 406, Form 27) named component_pkg_type.

The Generic Property Entity (Type 406, Form 27) named component_tech_type.

The Generic Property Entity (Type 406, Form 27) named component_layout_surface.

The Generic Property Entity (Type 406, Form 27) named component_height.

The Generic Property Entity (Type 406, Form 27) named component_max_size.

The Generic Property Entity (Type 406, Form 27) named component_outline_overhang.

In addition, the Subfigure Definition depicting the Mechanical Part may reference the following LEP properties:

Region Restriction Property Entity (Type 406, Form 2), and

Drilled Hole Property Entity (Type 406, Form 6).

The Singular Subfigure Instance Entity (Type 408) locating the Mechanical Part may reference any of the following three LEP Generic Properties (Type 406, Form 27):

$$
\begin{aligned}
& \text { component_preplace, } \\
& \text { therm_cond, and } \\
& \text { therm_r }
\end{aligned}
$$

\subsubsection{LEP Entity Usage for Physical PWB Representation}

The PWB from which a PCA is assembled may be an integral part of the assembly file/drawingg or may be a separate file/drawing that is called out (in the drawing hierarchy) by the assembly drawing. This Section is applicable to the latter case, and the traces, vias, drilled holes and other features applicable to the PWB. All components, Traces, Vias, (see Section 5.3.2.) and other entities are subordinate to the LEP PWB. 


\subsubsection{LEP Physical PWB Definition}

Entities required:

A Network Subfigure Definition Entity (Type 320) shall depict the Physical PWB. Each Network Subfigure Definition shall reference as many LEP Geometry Entities as needed to depict the PWB. Each Network Subfigure Definition may reference many of the properties listed below. Conformance to the LEP AP does not require any Text Display Template Entities (Type 312) in the representation of the PWB. At least one Connect Point Entity (Type 320) shall provide a means of connecting signals to "pin1" of the PWB.

\section{Geometry -}

Any LEP Geometry Entity may appear in conformant files. All geometry shall lie entirely in the Z $=0.0$ plane. There shall be one Closed Curve defining the LEP outline. The Closed Curve shall reference the "pwb_physical_outline" property. LEP properties shall assign functionality to any additional curves. Any of the LEP Geometry Entities may represent deposited?-Traces.

Annotation -

The LEP Physical PWB Definition (Type 320) may reference a General Note Entity (Type 212, Form 0).

\section{Structure -}

Network Subfigure Definition Entity (Type 320) depicts the LEP itself. Additional Network Subfigure Definitions and Instances may represent electrical Components, and Vias. Subfigure Definitions and Singular Subfigure Instances may represent Padstacks and mechanical parts.

\section{Associativities -}

Conformance to the LEP AP does not require any Associativities in the representation of the Definition.

\section{Properties -}

Mandatory Properties:

The Closed Curve depicting the LEP Outline shall reference the following properties:

The Generic Property Entity (Type 406, Form 27) named date.

The Generic Property Entity (Type 406, Form 27) named pwb_physical_outline.

The Generic Property Entity (Type 406, Form 27) named schematic_number.

The Generic Property Entity (Type 406, Form 27) named pwb_number.

The Generic Property Entity (Type 406, Form 27) named assembly_number.

Each collector of Geometry representing conductive Joins in the form of Traces shall reference the following property.

Line Widening Property Entity (Type 406, Form 5)

Level Function Property Entities (Type 406, Form 1) shall ascribe functionality (such as silkscreen layer) to various IGES levels.

Optional Properties:

The Network Subfigure Definition Entity (Type 320) modeling the LEP Physical PWB may refer- 
ence the following property. [?]

\subsubsection{LEP Physical PWB Instance}

\section{Entities required:}

The Network Subfigure Instance Entity (Type 420) locates the Physical PWB Instance occurrence and provides a way to connect to its ports. A Connect Point Entity (Type 132) models each port (e.g., Pin or Pad on an edge connector).

\section{Geometry -}

A Connect Point Entity (Type 132) having a Generic Property Entity (Type 406, Form 27) named pad_tech_type shall represent each Component Pin or each Component Pad on the Physical PWB. That property shall specify whether the Pad or Pin is of type edge_finger_pad, connector_socket, or connector_pin.

One Connect Point shall appear in the Network Subfigure Instance for each Pad, Pin or Socket in the referenced Network Subfigure Definition, even if the Pad, Pin or Socket is not used.

Annotation -

Conformance to the LEP AP does not require any Annotation Entities in the representation of the Physical PWB Instance. Stand-alone annotation may denote information on the LEP such as part_number, silk-screen, reference designator, or Pin number.

Structure -

The Network Subfigure Instance Entity (Type 420) shall reference one and only one Network Subfigure Definition Entity (Type 320). The Instance shall also specify the location of the Physical LEP with respect to the origin of Model Space.

The Network Subfigure Instance shall reference one or more Connect Point Entities (Type 132). Associativities -

The LEP Physical PWB Definition (Type 320) shall reference one Flow Associativity Entity (Type 402, Form 18) for each Join on the PWB. The Flow Associativity shall reference each Trace, filled area, Via, and Component Pin that is a member of the Join carrying the corresponding signal.

\section{Properties -}

Mandatory Properties:

The Network Subfigure Instance Entity (Type 420) representing the LEP Physical PWB Instance shall reference the following properties:

Part Number Property Entity (Type 406, Form 9).

Generic Property Entity (Type 406, Form 27) named top_level_assembly_instance.

Optional Properties:

The Network Subfigure Instance Entity (Type 420) may reference any of the following six LEP Generic Property Entites (Type 406, Form 27):

abs_voltage_max

tolerance 
therm_cond

therm_jc

therm_r

junction_max_t

Table 6-5. DE Settings of LEP Instance Entities in a PDQ IGES File

\begin{tabular}{|ll|l|l|}
\hline \multicolumn{1}{|c|}{ LEP Entity - DE Field } & $\begin{array}{c}\text { Network } \\
\text { Subfigure } \\
\text { Definition }\end{array}$ & $\begin{array}{c}\text { Network } \\
\text { Subfigure } \\
\text { Instance }\end{array}$ \\
\hline \hline Line Font & $(4)$ & 0,1 & 0,1 \\
\hline Level & $(5)$ & 0 to 1024 & 0 to 1024 \\
\hline View & $(6)$ & 0 & 0 \\
\hline Transformation Matrix & $(7)$ & 0 & 0, pointer \\
\hline Status; Blanked & $(9.1)$ & 00 & 00 \\
\hline Status; Subordinate & $(9.2)$ & 01 & 00 \\
\hline Status; Entity Use & $(9.3)$ & 02 & 00 \\
\hline Status; Hierarchy & $(9.4)$ & 01 & 01 \\
\hline Line Weight & $(12)$ & per IGES & per IGES \\
\hline Color & $(13)$ & 0 to 8 & 0 to 8 \\
\hline
\end{tabular}

The PDQ LEP representation in the IGES file shall have a Model space scale (Global index 13) of 1.0. A Transformation Matrix (needed for rotation) shall have no translation component.

\subsubsection{LEP Physical Flow Associativity (Signal/netlist)}

Each Flow Associativity Entity (Type 402, Form 18) models a distinct Signal.in a Netlist. Each Signal is insulated from all others. The Netlist is a report of all the signals in atparticular (sub)assembly of a product. Each LEP Connect Point Entity (Type 132) represents a port of a symbol in a schematic or a Pin/Pad of a physical Component. Thus, at least two types of Netlist exist for each LEP. IGES permits both logical and physical Flow Associativities to exist in the same file. The presence of both a logical and a physical netlist in the same file shall not be conformant to this AP. This discussion is limited to the physical realm.

Entities required:

Flow Associativity Entity (Type 402, Form 18), Connect Point Entity (Type 132), and LEP Join Geometry (below).

\section{Geometry -}

In the Physical realm, each Connect Point Entity (Type 132) represents a Component Pin or a 
Component Pad or a stand-alone Via. The Join geometry, representing the electrical connections between and among Connect Points, may be selected from the following LEP Geometry Objects: Line Entity (Type 110), Arc Entity (Type 100), full Circular Arc Entity (Type 100), Composite Curve Entity (Type 102), and Simple Closed Planar Curve Entity (Type 106, Form 63). Each Closed Curve shall reference one LEP Generic Property Entity (Type 406, Form 27) which defines its function. Each Line, Arc, or open Composite Curve shall reference two LEP properties: Line Widening Property Entity (Type 406, Form 5) and one LEP Generic Property Entity (Type 406, Form 27) named conductive_trace_function. All geometry shall lie entirely in the $Z=0.0$ plane.

Annotation -

Conformance to the LEP AP does not require any Annotation Entities in the representation of the Physical Signal.

The Flow Associativity Entity (Type 402, Form 18) collects the Connect Point Entities (Type 132) for each Signal. Network Subfigure Instance Entities (Type 420) shall reference each Connect Point Entity (Type 132) corresponding to a Pin or Pad of the Component represented in the Physical Netlist. LEP Geometry Entities or Flash Entities may depict the location of each Connect Point in the Netlist.

Associativities -

The Flow Associativity Entity (Type 402, Form 18) models each Signal in the Netlist.

Properties -

Mandatory Properties:

Each Closed Curve Join shall reference one LEP Generic Property Entity (Type 406, Form 27) which defines its function. The applicable LEP functions for Join geometry include: planar_shape_function \{signal, power, ground, .. \}. Each Line, Arc, or open Composite Curve Join shall reference two LEP properties: Line Widening Property Entity (Type 406, Form 5) and one LEP Generic Property Entity (Type 406, Form 27) named conductive_trace_function \{signal, power, ground, ... .

Optional Properties:

The Generic Property Entity (Type 406, Form 27) named pad_tech_type attached to the Connect Point may state whether the Pin is of SMT or through-hole design. The "pin1" Generic Property shall appear on those Connect Points bearing that designation.

\subsubsection{LEP Physical LEP Signal Carriers (Traces, Shapes) (1xx, 230)}

The Physical LEP Signal Carriers constitute the Join defined in IGES 3.6.3.2. Taken together, the Joins provide the connectivity that the Logical Netlist requires. Lines having specified width depict conductive Traces of similar width and a specified nominal thickness. Some Joins are irregularly shaped areas of conductive material of a specified nominal thickness.

Entities required:

The Join Geometry, depicting the electrical connections between and among Connect Points, may be selected from: Line Entity (Type 110), Arc Entity (Type 100), full Circular Arc Entity (Type 100), Composite Curve Entity (Type 102), Flash Entity (Type 125), and Simple Closed Planar 
Curve Entity (Type 106, Form 63).

\section{Geometry -}

LEP Join Geometry is defined above under Entities required. Any LEP Join Geometry Entities may appear in conformant files. All geometry shall lie entirely in the $\mathrm{Z}=0.0$ plane. Flash Entity (Type 125) shall not reference a Transformation Matrix.

\section{Annotation -}

The Closed Curve(s) enclosing the conductive areas of LEP Joins shall (each) reference a Sectioned Area Entity (Type 230). That Sectioned Area shall reference a Generic Property Entity (Type 406, Form 27) named planar_shape_function \{signal, power, ground, ... . . No other Annotation Entities shall appear as part of a Physical Signal/netlist representation.

Structure -

LEP Physical PWB Signal Carriers shall reference no structure entities other than the Flow Associativity.

\section{Associativities -}

LEP Physical PWB Signal Carriers shall reference one Flow Associativity Entity (Type 402, Form 18) for each Join.

\section{Properties -}

Mandatory Properties:

Each Closed Curve Join shall reference one LEP Generic Property Entity (Type 406, Form 27) named planar_shape_function which defines its function. Applicable LEP functions for Join geometry include: signal, power, and ground.

Each Line, Arc, or open Composite Curve Join shall reference two LEP properties:

\section{Line Widening (type 406 form 5) and}

LEP Generic Property (type 406 form 27) named conductive_trace_function.

The Generic Property Entity (Type 406, Form 27) named pad_tech_type attached to the Connect Point shall state whether the Pin is of SMT or through-hole design.

The "pin1" Generic Property shall appear on those Connect Points bearing that designation.

\subsubsection{LEP Physical PWB Features: Fiducials}

A Fiducial provides a reference location for accurate positioning of other LEP features.

Entities required:

Any LEP Geometry CLOSED CURVE may depict the FIDUCIAL.

\section{Geometry -}

Any LEP Geometry Entity may appear in conformant files. All geometry shall lie entirely in the Z $=0.0$ plane. The Flash Entity (Type 125) shall not reference a Transformation Matrix.

\section{Annotation -}

Conformance to the LEP AP does not require any Annotation Entities in the representation of the FIDUCIAL. 
Structure -

Conformance to the LEP AP does not require any Structure Entities in the representation of the FIDUCIAL.

Associativities -

Conformance to the LEP AP does not require any Associativity Entities in the representation of the FIDUCIAL.

Properties -

Mandatory Properties:

The following properties shall be referenced by each Closed Curve representing a FIDUCIAL:

The Generic Property Entity (Type 406, Form 27) named fiducial having a first value from among (component/cluster/board/panel) and a second value from among (top/bottom)

\subsection{LEP Entity Usage for Specific Applications}

This section discusses tests of conformant files and their construction. For example, Netlist reports require Flow Associativities.

\subsubsection{LEP Entity Usage for Design/Engineering Applications}

\subsubsection{LEP Entity Usage for Netlist Reports}

The netlist is a report of the interconnection of all Component terminals (Pins or Pads) sharing the same signal. Notice this includes both the logical and physical forms. The logical form relates each signal in the database with all of the unique part_pins which are part of that signal. The physical form adds the $\mathrm{X}$, $\mathrm{Y}$ location of each part_pin, and the connection geometry (Traces). The presence of both a logical and a physical netlist in the same file shall not be conformant to this AP except for this application.

This application does require a PDQ representation.

Entities required:

The Flow Associativity models each Signal. The LEP Geometry Entities depict the shape of the Join entities in the Physical Netlist. The LEP Geometry Entities may each reference one Line Widening Property Entity (Type 406, Form 5) to convey Trace width.

\section{Geometry -}

\section{Logical:}

The logical form of the netlist shall use no geometry. Any geometry present shall be ignored.

Physical:

Any LEP Geometry Entity may appear in conformant files. All geometry shall lie entirely in the $Z=0.0$ plane. The Flash Entity (Type 125) shall not reference a Transformation Matrix. The geometry entities usually depict the Traces. Via depictions may employ geometry. A Connect Point Entity (Type 132) shall model each Via. 
Both:

Component through connections shall appear as Connect Points in Physical netlists. However, the physical characteristics of the Connect Point shall not appear in the logical form of the netlist.

Annotation -

The Flow Associativity Entity (Type 402, Form 18) parameter NAME1 shall provide the Signal name. The Connect Point Entity (Type 132) parameter CID shall provide the Pin name. The Network Subfigure Instance Entity (Type 420) parameter PRD shall provide the Reference Designator of the Component having the participating PIN.

\section{Structure -}

The Flow Associativity Entity (Type 402, Form 18) groups the Connect Point Entities (Type 132) for each Signal. Network Subfigure Instance Entities (Type 420) shall reference each Connect Point Entity (Type 132) corresponding to a PIN or Pad of the Component represented in the Physical Netlist and may do the same in the Logical Netlist. LEP Geometry Entities or Flash Entities may depict the location of each Connect Point in either Netlist.

\section{Associativities -}

The Flow Associativity Entity (Type 402, Form 18) shall model each Signal in either form of Netlist. IGES requires the TF (type of flow flag) to be set to 1 or 2 for each occurrence of the Flow Associativity when both logical (1) and physical (2) flows are present in one file. Corresponding signals reference each other using the Flow Associativity parameters NF and SPTR1 through SPTRNF in such cases.

\section{Properties -}

The Generic Property Entity (Type 406, Form 27) named pad_tech_type attached to the Connect Point may state whether the Pin is of SMT or through-hole design. The pin 1 Generic Property may appear on some of the appropriate Connect Points.

\subsubsection{LEP Entity Usage for Component Placement Reports}

Component placement refers to the assignment of Components to specific $\mathrm{X}, \mathrm{Y}$ locations on the LEP. At times, particular Components must occupy well cooled spots or must adjoin other Components. The LEP component_preplace Property may identify such concerns. Component placement is also known as Component layout or LEP layout.

This Component placement application does require a PDQ representation.

Entities required:

Network Subfigure Definition Entity (Type 320) and Instance Entity (Type 420) represent the electrical parts. Subfigure Definition Entity (Type 308) and Singular Subfigure Instance Entity (Type 408) represent the mechanical parts.

The Network (or Singular) Subfigure Instance Entity (Type 420 or Type 408) locates the LEP Component. The LEP CLOSED CURVE depicts the shape of the LEP Component Outline. The LEP Closed Curve shall reference one Generic Property Entity (Type 406, Form 27) named physical_outline. 


\section{Geometry -}

A LEP Closed Curve having a physical_outline Property shall model each LEP Component Outline. Any LEP Geometry may be part of the LEP Closed Curve subject to the usual IGES constraints.

\section{Associativities -}

Conformance to the LEP AP does not require any associativities in the representation of the LEP Component Outline.

\section{Annotation -}

Conformance to the LEP AP does not require any Annotation Entities in the representation of the LEP Component.

\section{Structure -}

The LEP Closed Curve may reference one or more Generic Property Entities t(ype 406, Form 27) named component_preplace, or component_instance_side in LEP AP conformant files.

\section{Properties -}

\section{Mandatory Properties:}

The Network (or Singular) Subfigure Instance Entity (Types 420 or 408) shall reference the following LEP properties:

The Generic Property Entity (Type 406, Form 27) named component_std_name.

The Generic Property Entity (Type 406, Form 27) named component_version.

The Generic Property Entity (Type 406, Form 27) named physical_outline.

The Generic Property Entity (Type 406, Form 27) named component_tech_type.

Optional Properties:

The Network (or Singular) Subfigure Instance Entities (Types 420 or 408) may reference the following LEP property:

The Generic Property Entity (Type 406, Form 27) named component_preplace, and [?]

\subsubsection{LEP Entity Usage for Bill of Materials Reports}

The Bill of Materials (BOM) report lists the Components which make up the LEP. The BOM lists part numbers, quantity used, and a brief description of the parts. Some BOMs also list reference designators. The report may include other semantic information as well. This BOM application does require a PDQ representation.

Entities required:

Network Subfigure Definition Entity (Type 320) and Instance Entity (Type 420) represent the electrical Components. Subfigure Definition Entity (Type 308) and Singular Subfigure Instance Entity (Type 408) represent the mechanical Components.

The Part Number Property Entity (Type 406, Form 9) attached to the Singular (or the Network) Subfigure Instance Entities (Type 408 or 420 ) provides the part number to the BOM report. The quantity used is calculated by the software. If needed, the PRD parameter of the Network Subfig- 
ure Instance Entity (Type 420) shall provide the Reference Designator data.

\section{Geometry -}

Any LEP Geometry Entities may be referenced by the Subfigure Definitions in the depiction of LEP Components.

\section{Annotation -}

Conformance to the LEP AP does not require any Annotation Entities in the representation of the LEP Component.

\section{Structure -}

The Network Subfigure Instance Entity (Type 420) shall reference one Network Subfigure Definition Entity (Type 320) and one or more BOM Properties for each electrical Component. The Singular Subfigure Instance Entity (Type 408) shall reference one Subfigure Definition Entity (Type 308 ) and one or more BOM Properties for each mechanical Component.

\section{Associativities -}

Conformance to the LEP AP does not require any associativities in the representation of the BOM.

\section{Properties -}

Mandatory Properties:

The Part Number Property Entity (Type 406, Form 9) shall provide part numbers for each Component. This property identifies 4 different part number families; the generic, military, vendor, and internal part numbers. The property shall provide at least one of these four part numbers for each Component.

Each Component instance represented by a Network Subfigure Instance Entity (Type 420) or a Singular Subfigure Instance Entity (Type 408) shall reference the following LEP properties:

The Part Number Property Entity (Type 406, Form 9).

Optional Properties:

Each Component instance represented by a Network Subfigure Instance Entity (Type 420) or a Singular Subfigure Instance Entity (Type 408) may reference the following LEP properties:

The Generic Property Entity (Type 406, Form 27) named:

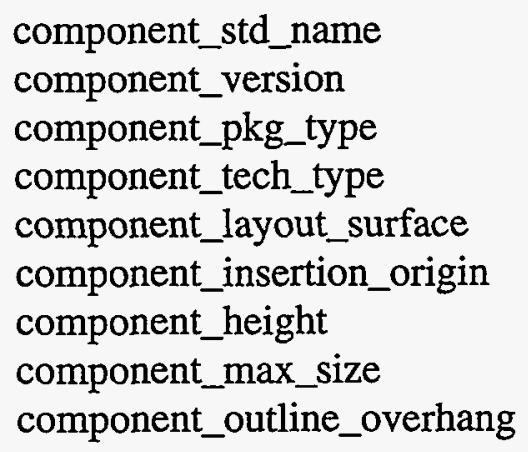




\subsubsection{LEP Entity Usage for Manufacturing Applications}

Usage rules are laid down for providing usable information to support Photo-lithography tools, LEP Testers, Numerically Controlled Component processing and Panelization of PWB details. Concepts for support of Wire Wrap is included and Labelmaker application may be added later.

\subsubsection{LEP Entity Usage for Photo-lithography Tools}

Photo-lithography in this application refers to the creation of the master artwork used to manufacture the physical bare PWB or other LEP. This includes the Traces, planes, and Vias. It also includes annotation such as reference designators, Pin numbers, Component outlines, part numbers, tooling holes, Fiducials, and labels.

This photolithography application does NOT require a PDQ representation.

Entities required:

Any LEP Geometry Entity may depict part of the Traces, Vias, and annotation graphics needed for this application. The General Note Entity (Type 212, Form 0) may provide the text strings needed for other annotation.

\section{Geometry -}

Any LEP Geometry Entity may appear in conformant files. All geometry shall lie entirely in the $Z$ $=0.0$ plane. The Flash Entity (Type 125) shall not reference a Transformation Matrix. Receiving systems may balk at geometry more complex than a Line Entity (Type 110), Circular Arc Entity (Type 100), Copious Data Entity (Type 106), Composite Curve Entity (Type 102), or Flash Entity (Type 125).

Single entities shall depict each Fiducial and each tooling mark. The (full) Circular Arc Entity (Type 100) or the Flash Entity (Type 125) may serve some of these needs. The others may require a Composite Curve Entity (Type 102) or the Copious Data Entity (Type 106, Form 36). The Composite Curve shall gather the constituent entities when multi-entity representations are needed. That Composite Curve shall reference any properties providing semantics such as the Generic Property Entity (type 406, Form 27) named "fiducial".

\section{Annotation -}

Closed Curves may annotate the LEP by stylized depictions of Component outlines. The General Note Entity (Type 212, Form 0) may present Reference Designator and Pin text plus LEP identifiers.

\section{Structure -}

The simpler the structure, the better. Copious Data Entity (Type 106) may depict objects representable by polygons and Composite Curve Entity (Type 102) Entities may group Lines and Circular Arcs.

\section{Associativities -}

Conformance to the LEP AP does not require any associativities in the representation of the photo-tools.

\section{Properties -}

The Composite Curve Entity (Type 102), Circular Arc Entity (Type 100), Copious Data Entity 
Implementation \& Conformance Testing

(Type 106), Flash Entity (Type 125) or the Line Entity (Type 110) shall reference the following LEP properties:

The Line Widening (Type 406, From 5) Property shall specify nominal Trace width. Thus, it shall not widen or narrow as the underlying geometry gets scaled.

The Generic Property Entity (Type 406, Form 27) named "fiducial" shall tag referenced objects.

\subsubsection{LEP Entity Usage for LEP Test Aids}

This application involves provision of information to support automated testing of an LEP. The tester needs probe locations tied to signal names. The tester also needs locations of Components which might interfere with probe insertion or removal. The object descriptions needed include those of the Traces, planes, padstacks and Vias. It also includes annotation such as Reference Designators, Pin numbers, Component outlines, Part Numbers, tooling holes, Fiducials, and labels.

This application does require a PDQ representation.

Entities required:

The Flow Associativity Entity (Type 402, Form 18), the Connect Point Entity (Type 132), and the Network Subfigure Instance Entity (Type 420) provides the "PDQ intelligence" (connectivity information) needed for this application. Network Subfigure Definition Entity (Type 320) and Instance Entities (Type 420) represent the electrical Components. If needed, the PRD parameter of the Network Subfigure InstanceEntity (Type 420) shall provide the Reference Designator data.

Any LEP Geometry Entity may depict part of the Traces, Vias, and annotation graphics needed for this application. The General Note Entity (Type 212, Form 0) may provide the text strings needed for other annotation.

\section{Geometry -}

A LEP Closed Curve having a physical_outline Property shall model each LEP Component Outline. Any LEP Geometry may be part of the LEP Closed Curve. All geometry shall lie entirely in the $Z=0.0$ plane.

Single entities shall depict each Via, each connect point, each Fiducial and each tooling mark. The (full) Circular Arc Entity (Type 100) or the Flash Entity (Type 125) may serve.some of these needs. The others may require a Composite Curve Entity (Type 102) or the Copious Data Entity (Type 106, Form 63). The Composite Curve shall group the constituent entities when multi-entity icons are needed. That Composite Curve shall reference any properties providing semantics such as the Generic Property Entity (Type 406, Form 27) named "fiducial".

The Sectioned Area Entity (Type 230) shall depict filled areas such as irregularly shaped signal planes, ground planes or power planes. Only the (full) Circular Arc Entity (Type 100), Composite Curve Entity (Type 102), and Simple Closed Planar Curve Entity (Type 106, Form 63) shall be filled by the Sectioned Area Entity. Only the unfilled Fill Pattern (0) and the solid filled Fill Pattern (19) of the Sectioned Area Entity (Type 230) shall appear in conformant files.

Annotation -

Network Subfigure Instance Entities (Type 420) will provide information on Component location, Pin location, Signal Name, Reference Designator, Part number, and Pin text. 
The Flow Associativity Entity (Type 402, Form 18) parameter NAME1 shall provide the Signal name. The Connect Point Entity (Type 132) parameter CID shall provide the Pin Name. The Network Subfigure Instance Entity (Type 420) parameter PRD shall provide the Reference Designator of the Component having the participating Pin.

\section{Structure -}

The Network Subfigure Instance Entity (Type 420) shall reference one Network Subfigure Definition Entity (Type 320) and one or more Test Properties for each electrical Component. The Network Subfigure Instance shall also reference one Connect Point Entity (Type 132) for each corresponding Connect Point in the referenced Network Subfigure Definition Entity (Type 320). Each Connect Point Entity (Type 132) shall provide its Pin Name in parameter PRD. Some instanced Connect Points Entities (Type 132) may model Vias rather than Component Pins. (Vias have no owner. In other words, the PSFI parameter of the Tyoe 132 has no valid pointer.) Each owned and instanced Connect Point Entity. (Type 132) shall provide a Pin name. The combination of the Reference Designator and the Pin name shall be unique within the IGES file in which it appears. Each instanced Connect Point Entity (Type 132) may reference a Generic Property Entity (Type 406, Form 27) stating whether test probing is allowable or not. Each instanced Connect Point which does not reference either of those properties should not be probed. Each Flow Associativity Entity (Type 402, Form 18) shall provide a Signal Name (in NAME1). Each Flow Associativity shall reference all the Connect Points carrying the Signal Name found in NAME1.

\section{Associativities -}

The Flow Associativity Entity (Type 402, Form 18) models each Signal.

\section{Properties -}

Mandatory Properties:

Each Connect Point Entity (Type 132) shall reference one of the following two LEP properties:

The Generic Property Entity (Type 406, Form 27) name:

allowable_test_point

or (the default) not_allowable_test_point

The Network Subfigure Instance shall reference the following LEP properties:

The Generic Property Entity (Type 406, Form-27) named: component_instance_side.

Optional Properties:

The Connect Point Entity (Type 132) may reference the following LEP properties:

The Generic Property Entity (Type 406, Form 27) name: pin1.

The Closed Curve may reference the following LEP properties:

The Generic Property Entity (Type 406, Form 27) name: fiducial.

\subsubsection{LEP Entity Usage for Numerically Controlled (NC) Production Files}

Component Insertion, Component Pick and Place, and Component Verification and a concept for Wire Wrap are discussed below. Discussions of Label making may be added at some later date. The LEP AP files described below may be converted to MIL-D-28000 Class IV files as needed. 


\subsection{LEP Entity Usage for Component Insertion}

This Component Insertion application does require a PDQ representation.

Component Insertion refers to the process of inserting through-hole Components of one or more distinct types into the PWB. All Components within each type are the same as far as the inserter is concerned. For example, one type may be a $10 \mathrm{~K} 1 / 4 \mathrm{w} 5 \%$ resistor and another type may be a $22 \mathrm{~K}$ 1/4w $10 \%$ resistor. Each type must be distinctly identified. The most common method to do this is with a Part Number Property Entity (Type 406, Form 9), using the internal Part Number Property Entity (Type 406, Form 9) value.

Automated insertion is concerned with two distinct rotations. The first rotation occurs during physical layout when placing the part from the LEP design library. For example, an axial Component is usually mounted with its axis parallel to the plane of the LEP. With the axis perpendicular to the plane of the LEP, the Component has been rotated 90 degrees (or 270 degrees.) (The amount of rotation (90 or 270) matters little if the Component is non-polarized. For a diode or a polarized capacitor, though, it is important.) These rotations are communicated to the insertion machinery by means of the Generic Property Entity (Type 406, Form 27) named component_placement_angle. The Transformation Matrix Entity (Type 124) is not applicable in such cases because there is no fixed frame of reference.

The other kind of rotation is beyond the scope of this AP. It tracks the motions of the head of the inserter and of the Component in the feeder.

Entities required:

The Network Subfigure Instance Entity (Type 420) models the Component inserted. It shall reference a Network Subfigure Definition containing a CLOSED CURVE which depicts the shape of the Component outline after insertion. It may also reference a Transformation Matrix Entity (Type 124) to model the rotation of the Component into position in model space. The Transformation Matrix shall not apply translations of the Component in the plane of the LEP. The X and Y coordinates within the Network Subfigure Instance Entity (Type 408) shall determine the lateral position of the Component on the LEP. The Network Subfigure Instance Entity (Type 420) shall reference a Generic Property Entity (Type 406, Form 27) named component_instance_side to state whether the Component is mounted on the top or the bottom of the LEP. Else, the default position is on top of the PWB. The Subfigure Instance may also reference a Generic Property Entity (Type 406, Form 27) named component_instance_rotation if needed. The Network Subfigure Instance Entity (Type 420) shall reference other properties to provide semantic information to the inserter.

\section{Geometry -}

One CLOSED CURVE shall define the outline of the Physical Component in the X, Y plane of the LEP. Only the (full) Circular Arc Entity (Type 100), the Composite Curve Entity (Type 102), and the Simple Closed Planar Curve Entity (Type 106, Form 63) may appear as entities which define Component outlines. Each such CLOSED CURVE shall reference one LEP Property which defines its function. For this application, the LEP function needed is the physical_outline.

Any LEP Geometry Entity may appear as part of the Component Geometry. All LEP Geometry shall lie entirely in the $Z=0.0$ plane.

\section{Annotation -}

Conformance to the LEP AP does not require any Annotation Entities in the representation of the 
Physical Component.

Structure -

The Network Subfigure Instance Type (Type 420) shall reference one Network Subfigure Definition Entity (Type 320) and one or more Component Insertion Properties for each electrical Component to be inserted. The Network Subfigure Definition (together with its Transformation Matrix) control the orientation and position of insertion.

Associativities -

Conformance to the LEP AP does not require any associativities in the representation of the Physical Component to be inserted.

Properties -

Mandatory Properties:

The Network Subfigure Definition Entity (Type 320) shall reference the following LEP properties:

The Generic Property Entity (Type 406, Form 27) named:

component_insertable

component_insertion_origin

The Network Subfigure Instance Entity (Type 420) shall reference the following LEP properties:

The Generic Property Entity (Type 406, Form 27) named:

component_insertion_force

component_insertion_height

The Connect Point Entity (Type 132) shall reference one of the following two LEP properties:

The Generic Property Entity (Type 406, Form 27) name:

allowable_test_point

or (the default) not_allowable_test_point

Optional Properties:

The Network Subfigure Definition Entity (type 320) may reference the following LEP properties:

The Generic Property Entity (Type 406, Form 27) named:

component_max_size

The Network Subfigure Instance Entity (Type 420) may reference the following LEP properties:

The Generic Property Entity (Type 406, Form 27) named:

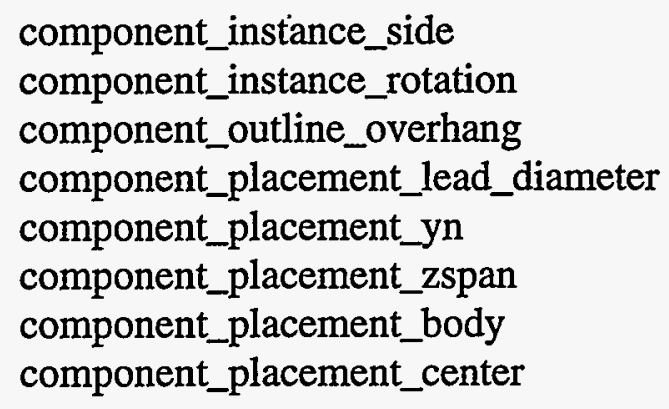




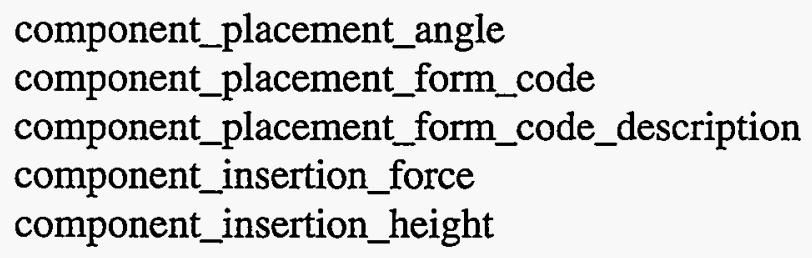

\subsection{LEP Entity Usage for Component Pick and Place}

This application does require a PDQ representation.

Component Pick and Place refers to the process of pasting SMT Components of one or more distinct types onto the LEP. All Components within each type are the same as far as the Pick and Place system is concerned. For example, one Component type may be a memory chip and another Component type may be a multiplexer chip. Each Component type must be distinctly identified. The most common method to do this is with a Part Number Property Entity (Type 406, Form 9), using the internal Part Number Property value.

Automated Pick and Place is concerned with two distinct rotations. The first rotation occurs during physical layout when placing the part from the LEP design library. For example, an MCM chip Component is usually mounted with Pin 1 in the upper left hand corner. With Pin 1 in the upper right hand corner, the Component has been rotated 270 degrees. These rotations are communicated to the placement machinery by means of the Generic Property Entity (Type 406, Form 27) named component_placement_angle. The Transformation Matrix Entity (Type 124) is not applicable in such cases because there is no fixed frame of reference.

The other kind of rotation is beyond the scope of this AP. It tracks the motions of the head of the Pick and Place system and of the Component in the Pick mechanism.

Entities required:

The Network Subfigure Instance Entity (Type 420) models the Component placed. It shall reference a Network Subfigure Definition containing a CLOSED CURVE which depicts the shape of the Component outline after placement. It may also reference a Transformation Matrix Entity (Type 124) to model the rotation of the Component into position in model space. The Transformation Matrix shall not apply translations of the Component in the plane of the LEP. The X and Y coordinates within the Network Subfigure Instance Entity (Type 408) shall determine the lateral position of the Component on the LEP. The Subfigure Instance shall reference a Generic Property Entity (Type 406, Form 27) named component_instance_side to state whether the Component is mounted on the top or the bottom of the LEP. If it does not, the default position is on top of the LEP. The Subfigure Instance may also reference a Generic Property Entity (Type 406, Form 27) named component_instance_rotation if needed. The Subfigure Instance shall reference other properties to provide semantic information to the Pick and Place system.

Geometry -

One Closed Curve shall depict the outline of the Physical Component in the X, Y plane of the LEP. Only the (full) Circular Arc Entity (Type 100), the Composite Curve Entity (Type 102), and the Simple Closed Planar Curve Entity (Type 106, Form 63) may appear as entities which define Component outlines. Each Closed Curve shall have one LEP Property attached to it which defines its function. For this application, the LEP function needed is the physical_outline.

Any LEP Geometry Entity may appear as part of the Component geometry. All LEP Geometry 
shall lie entirely in the $Z=0.0$ plane.

Annotation -

Conformance to the LEP AP does not require any Annotation Entities in the representation of the Physical Component.

\section{Structure -}

The Network Subfigure Instance Entity (Type 420) shall reference one Network Subfigure Definition Entity (Type 320) and one or more Component Insertion Properties for each electrical Component to be placed. The Network Subfigure Definition (together with its Transformation Matrix) control the orientation and position of adhesion.

Associativities -

Conformance to the LEP AP does not require any associativities in the representation of the Physical Component to be placed.

Properties -

Mandatory Properties:

The Network Subfigure Definition Entity (Type 320) shall reference the following LEP properties:

The Generic Property Entity (Type 406, Form 27) named:

component_layout_surface (top/bottom)

component_insertable (yes/no)

The Network Subfigure Instance Entity (Type 420) shall reference the following LEP properties:

Part Number Property Entity (Type 406, Form 9).

The Generic Property Entity (Type 406, Form 27) named:

$$
\begin{aligned}
& \text { component_placement_force } \\
& \text { component_placement_height } \\
& \text { component_placement_angle } \\
& \text { component_placement_depth_stop } \\
& \text { component_placement_yn }
\end{aligned}
$$

Optional Properties:

The Network Subfigure Definition (type 320) may reference the following LEP properties:

The Generic Property Entity (Type 406, Form 27) named:

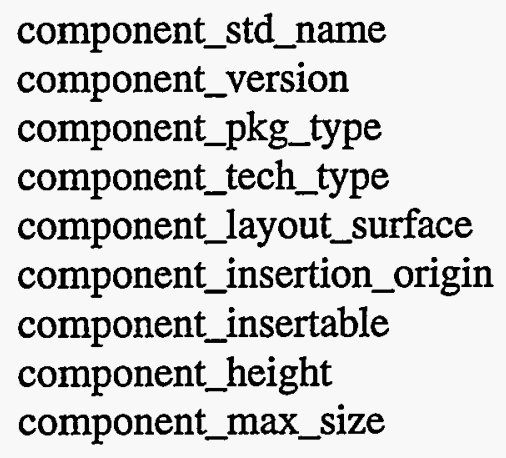


component_outline_overhang

The Network Subfigure Instance Entity (Type 420) may reference the following LEP properties:

The Generic Property Entity (Type 406, Form 27) named:

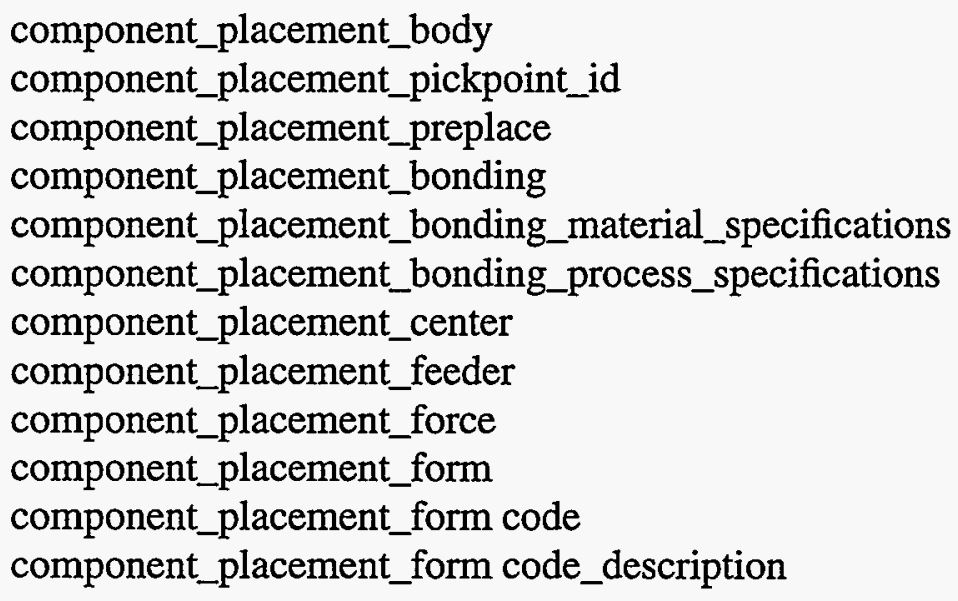

\subsection{LEP Entity Usage for Component Verification}

This application verifies the correct Components, properly oriented, are correctly positioned on the LEP. It needs Component outline (a Closed Curve), Part number, X, Y position, and Pin 1 property for each Component occurrence. This application does NOT require a PDQ representation.

Entities required:

The Network Subfigure Instance Entity (Type 420) models the LEP Component. The CLOSED CURVE depicts the shape of the LEP Component. The Network Subfigure Instance Entity (Type 420) shall reference one or more LEP Component Properties in LEP AP conformant files.

\section{Geometry -}

A Closed Curve having a placement_outline Property shall model each LEP Component in LEP AP conformant files.

Annotation -

Conformance to the LEP AP does not require any Annotation Entities in the representation of the LEP Component representation.

\section{Structure -}

The Network Subfigure Instance Entity (Type 420) shall reference one Network Subfigure Definition Entity (Type 320). The Network Subfigure Definition Entity (Type 320) shall reference a CLOSED CURVE. The Definition shall reference one or more LEP Component Properties in LEP AP conformant files.

\section{Associativities -}

Conformance to the LEP AP does not require any associativities in the representation of the LEP Component. 
Properties -

Mandatory Properties:

The Network Subfigure Instance Entity (Type 420) shall reference the following LEP properties:

Part Number Property Entity (Type 406, Form 9).

The Generic Property Entity (Type 406, Form 27) named placement_outline.

The proper Connect Point Entity (Type 132) of each Component shall reference the Generic Property Entity (Type 406, Form 27) named pin1.

\subsection{LEP Entity Usage for Wire Wrap}

A simplified model of a 2 layer board will suffice for most wire wrap situations; for more complex designs you will see a multilayer PWB (with or without some mounted components) with compliant pins friction-fit into the through hole barrels of the board. Wire wrap will usually extend off the board typically to connectors with wire wrap pins (in most cases, it is illegal or inadvisable to solder the solid wire used for wire wrap). Occasionaly, the motherboard will also include some flex-tape arrangement also (this is infrequent, but a possibility).

For the wire wrap board, the PWB, Section 6.5.4. is applicable; however the traces and associated Line Widening Property Entity (Type 406, Form 5) are omitted. The completed wire wrap representation will contain components whose pins are located only where a wire wrap pin exists. There are various CAE techniques employed to guide the layout of components on the PWB such as grids and wire wrap board graphics.

For the components, Section 6.5.2. is applicable; a padstack may be used, however the usual PWB lands may be omitted.

The netlist is merged such that the component pins are associated with a signal, thus Section 6.6.1.1. is applicable. Routing is routinely done for many wire wrap situations (especially for 3-D harnes situations, where the final assembly has connections in multiple planes and a "twist" has to be thrown in), but seldom is any of the routing described in any CAD data. The wire wrap product definition may be released or archived at this point.

The wire wrap product is completed by extraction (from the CAE system or from an IGES LEP file) of the netlist with the X, Y locations recorded for each "terminal" in the netlist. The extracted list is "post-processed"; sorted into a wire wrap-sequence order as determined to be optimum for the wire wrap machine used, and the wire lengths (usually precut to the machine requirements) selected. The completed wire wrapped board is continuity-tested (see Section 6.6.2.2.), and the components are inserted (for automated insertion Section 6.6.2.3.1. applies) in the identified locations.

Note that there is little control on the "path" taken by the wires; thus the wire wrap method of assembly is usually limited to low production runs, such as prototypes and special purpose test equipment. Some computer backplanes have utilized wire wrap construction because of the relatively low distributed capacitence between wires and the possibility to make field modifications to the connections. 


\subsection{LEP Entity Usage for Labelmaker UNDER CONSTRUCTION.}

\subsubsection{LEP Entity Usage for Panelization}

Panelization involves laying out two or more LEP assembly outlines on the surface of a substrate panel for processing efficiency considerations. Fiducials and test coupons are usually added to the layout design to enable better Quality Control. The information needed is the LEP outline (Singular Subfigure Definition Entity (Type 308) referencing a CLOSED CURVE having a Generic Property Entity (Type 406, Form 27) named pwb_physical_outline Property, and those CLOSED CURVES which reference fiducial/board and fiducial/panel Properties.

This application does NOT require a PDQ representation.

Entities required:

One Closed Curve shall define the outline of the PWB. Two or three other CLOSED CURVES depicting Fiducials are also needed. A Group Associativity Entity (Type 402, Form 7) without backpointers shall reference each entity that is to appear in a given panel.

\section{Geometry -}

Fiducials may be represented by single entities, such as the Flash Entity (Type 125), or multiple entities such as circles depicted by many short lines. Multi-entity Fiducials shall be subordinate to a Composite Curve Entity (Type 102). The single entity or Composite Curve Entity (Type 102) shall reference a Fiducial Property.

\section{Annotation -}

Conformance to the LEP AP does not require any Annotation Entities in the representation of the CLOSED CURVE depicting the PWB.

Structure -

One CLOSED CURVE shall define the outline of the PWB. Only the (full) Circular Arc Entity (Type 100), the Composite Curve Entity (Type 102), and the Simple Closed Planar Curve Entity (Type 106, Form 63) may appear as entities which define the PWB outline. That CLOSED CURVE shall reference the Generic Property Entity (Type 406, Form 27) named top_level_assembly_instance. The other CLOSED CURVES needed shall reference the Generic Property Entity (Type 406, Form 27) named "fiducial" and having the value "board" or the one having the value "panel" as described below.

\section{Associativities -}

A Group Associativity, without backpointers (Type 402, Form 7) shall reference each entity that is to appear in a given panel.

Notice that this associativity does not require backpointers from each entity back to the Group Associativity.

\section{Properties -}

Mandatory Properties:

The CLOSED CURVE(s) depicting fiducials outside the PWB outlines shall reference 
The Generic Property Entity (Type 406, Form 27) named fiducial/panel.

The other CLOSED CURVES depicting fiducials shall reference

The Generic Property Entity (Type 406, Form 27) named fiducial/board.

A Generic Property Entity (Type 406, Form 27) named "panel_offset" shall be attached to each associativity to mark it as a panel associativity. In addition, the panel_offset property has two real values, $\mathrm{X}$ and $\mathrm{Y}$, that specify the offset for this pattern in relation to the panel.

\subsubsection{LEP Entity Usage for Drafting/Documentation Applications}

Documentation of the LEP is primarily a manual operation. However, there are ways to automate parts of the documentation preparation processes. The differences between the information needed for documentation versus that needed for automated and semi-automated processes are discussed below. Following that is usage information about Assembly Documentation and a reference to an AP for Technical Illustrations. For this version, the discussion on entities to support Fabrication documentation is lamentably sketchy.

\subsubsection{Major differences between the documentation and the PDQ model of a conformant LEP AP -}

The PDQ model of a conformant LEP AP is at a specific orientation with a scale of 1.0. The documentation may be at any rotation(s) with any scale (e.g., to show fine detail). The picture may be cut into two or more sheets. One half of a scaled drawing then appears on one sheet, and the other half on the second sheet.

The PDQ model of a conformant LEP AP usually contains only a few annotation entities. The documentation contains many annotation entities, such as dimensioning and labeling.

The PDQ model of a conformant LEP AP exists in model space. The documentation often has many distinct views and may have a drawing space for each drawing entity.

\subsubsection{Changes and additions to PDQ LEPAP}

Changes to the DE for Documentation files:

No hierarchy is needed,

Line font may have more variability than eight-colors,

Line thickness may have more variability, and

Components need not be subordinate.

Entities (Type 1xx) or entity forms beyond the LEP AP may be needed.

Geometry entities have been added:

Unbounded Plane Entity (Type 108) (only as a clipping plane to a View Entity (Type 410) has been added for documentation). Additional forms of the Copious Data Entity (Type

106) include Forms 20 and 21, 31-38, and 40.

Dimensioning entities have been added:

All 200 series entities are permitted in documentation files.

Other entities have been added for documentation files: 
Full color (including the Color Definition Entity (Type 314)); dimensions of various types; the View Entity (Type 410) and Drawing Entity (Type 404); plus various Properties- (e.g., for Units and Size).

The Flow Associativity Entity (Type 402, Form 18) is not needed for documentation files.

Type 420/320/132 entities:

Strictly speaking, these connectivity entities are not needed for documentation files. For some users though, the loss of PDQ information may outweigh the ease of implementation.

Documentation of the LEP includes:

Drafting/Dimensioning usually provides overall LEP dimensions, plus dimensions relating various LEP features such as tooling holes and mounting hardware.

\subsubsection{LEP Entity Usage for Fabrication Documentation}

This documentation includes: Fabrication instructions including layer order, substrate material and thickness, special processing notes, and registration Fiducials.

Production Assembly information tells how to build a specific LEP product using detailed step by step instructions for a given manufacturing process.

\subsubsection{LEP Entity Usage for Assembly Documentation}

Production Assembly information tells how to build a specific LEP product using detailed step by step instructions for a given manufacturing process.

One common method used in manufacturing is the "station/operation" approach. This method divides the task of populating a LEP into several discrete steps. Each step begins with a check of the work done in the previous step. Then the task(s) of the current step are performed and the partial assembly is passed onto the correct location for the next step. For example, step one might inspect the unpopulated LEP for damage or irregularities. If the LEP seems good in that regard, it is placed in a holder, and then five resistors having identical part numbers are inserted into the PWB. Step two begins with an inspection to ensure the five parts supposedly added in step 1 are in position, and that each has (or could have) the correct part number. The operator performing step 2 then inserts six capacitors and four more resistors. Step 3 begins by inspecting the four resistors. Then the six capacitors are checked for polarity as well as correct part number and location.

The elapsed time to perform the tasks at each station may be adjusted to minimize bottlenecks. Each station is furnished with a color coded picture, indicating which parts added at the previous station are to be inspected, and which parts are to be added at this station. Manual creation of these process pictures may take quite a bit of resources and time, or, it may be automated using a PDQ LEP database.

If this process sheet creation application will not be automated, then MIL-D-28000 Class II, the Engineering Drawing subset may be invoked to assure conformance of any IGES drawing files created to provide color coded pictures at each station.

If this process sheet creation application will be automated, a PDQ LEP AP or data base may well 
be employed. Notice that the final IGES files that are generated by the automated process may need to be converted to MUL-D-28000 Class II IGES files. Colors and fill patterns may be assigned during that conversion process.

One additional file is required by the automated process. It is a file that tells which parts are placed at which station.

An algorithm for automating the sheet creation process appears in Appendix B of this AP.

\subsubsection{LEP Entity Usage for LEP Technical Illustrations}

The CALS MIL-D-28000 Class I Technical Illustrations conformance requirements for Technical Publications shall apply for LEP Technical Publications. The LEP Technical Illustrations application shall add no entities, nor impose changes to existing Class I entities.

\subsubsection{LEP Entity Usage for CAD to CAD exchange}

Modifying the LEP AP IGES file to make it more legible to a particular brand or version of receiving CAD system is outside the scope of this AP. Vendors products may exist in this area.

\subsubsection{LEP Entity Usage for Layer assignment}

The assignment of particular LEP features, such as LEP Layers, to specific IGES Levels is outside the scope of this AP. In this AP, most semantic information is conveyed through the use of IGES Properties (Type 406).

\subsubsection{LEP Entity Usage for Color}

The custom of using colors to convey semantic information is outside the scope of this AP. A palette of 8 colors has been adopted to provide minimal visual variety.

\subsubsection{Semantic Properties for LEPApplications}

This AP requires the use of specific properties to communicate the intended use of some of the data encoded by means of the AIM. These properties are gathered into sections for particular Application Information Requirement areas and listed below. Each entry in the list is in the form of a string as it might appear in the Parameter Data of the Property Entity (Type 406). This serves to illustrate how name strings, (e.g., "3, 3Htop" for top) integers, (e.g., " 1,420 " for 420) and real numbers (e.g., "2, 0.250" for 0.25 ) may appear in the IGES file sent to the processor(s). The Generic Property Entities (Type 406, Form 27) are especially versatile in this regard. This versatility was one of the considerations in choosing this property over the older-but seldom implemented by ECAD vendors-Attribute Table (Type 322 and 422) mechanism.

Forms 1-3, 5, 6, 8-10, plus 24-27 of the IGES Property Entity (Type 406, Form nn) may be found in conformant files. This AP specifies which entities are to reference particular Properties whenever that seems feasible. Some Properties may stand alone in the LEP AP conformant files. Many Generic Property Entity (Type 406, Form 27) are introduced in this AP to convey important semantic information and data for LEP applications.

\section{List of New Level Function Properties for LEP Applications}

Processors should treat property text strings, both descriptions and values, as not case sensitive. 
Implementation \& Conformance Testing

$0=$ No value; $1=$ Integer; $2=$ Real; $3=$ Character string; $4=$ Pointer; $5=$ Not used; $6=$ Logical

Table 6-6. Level Function Properties (Type 406, Form3)

\begin{tabular}{|c|}
\hline Parameter Data (fragment) \\
\hline $\begin{array}{l}0,9 \text { Htrace_top } \\
0,9 \text { Htrace_bot } \\
0,7 \text { Htrace_2 } \\
0,7 \text { Htrace_n } \\
0,14 \text { Hsilkscreen_top } \\
0,14 \text { Hsilkscreen_bot }\end{array}$ \\
\hline
\end{tabular}


List of New Generic Properties for LEP Applications

Table 6-7. Generic Properties of Top Level LEP

\begin{tabular}{|c|c|c|c|c|}
\hline \multicolumn{5}{|c|}{ Parameter Data (fragment) } \\
\hline NP & Name & NV & TYP* & Value* \\
\hline $\begin{array}{l}1, \\
4, \\
4, \\
4, \\
6, \\
4, \\
4, \\
4, \\
4, \\
4, \\
4, \\
4, \\
4, \\
4, \\
4, \\
4, \\
4, \\
16,\end{array}$ & $\begin{array}{l}\text { 27Htop_level_assembly_instance ; } \\
\text { 16Hlep_assembly_tag, } \\
\text { 25Hprinted_wire_assembly_tag 1, } \\
\text { 32Hhybrid_microcircuit_assembly_tag, } \\
\text { 4Hdate, } \\
\text { 26Hcomponent_default_padstack, } \\
\text { 16Hschematic_number, } \\
\text { 15Hpwb_part_number, } \\
\text { 15Hhm__part_number, } \\
\text { 15Hmcm_part_number, } \\
\text { 15Hpwa_part_number, } \\
\text { 15Hpcb_part_number, } \\
\text { 15Hpca_part_number, } \\
\text { 17Hflexb_part_number, } \\
\text { 17Hflexc_part_number, } \\
\text { 24Hmicrostrip_b_part_number, } \\
\text { 23Hassembly_drawing_number, } \\
\text { 24Hlep_physical_orientation, } \\
\text { 20Hlep_design_thickness, }\end{array}$ & $\begin{array}{l}1, \\
1, \\
1, \\
1, \\
1, \\
1, \\
1, \\
1, \\
1, \\
1, \\
1, \\
1, \\
1, \\
1, \\
1, \\
7,\end{array}$ & $\begin{array}{l}3, \\
3, \\
3, \\
3, \\
3, \\
3, \\
3, \\
3, \\
3, \\
3, \\
3, \\
3, \\
3, \\
3, \\
3, \\
3, \\
3, \\
3, \\
1, \\
3, \\
2, \\
3, \\
1, \\
3, \\
3, \\
2, \\
3, \\
2, \\
3, \\
2, \\
3,\end{array}$ & 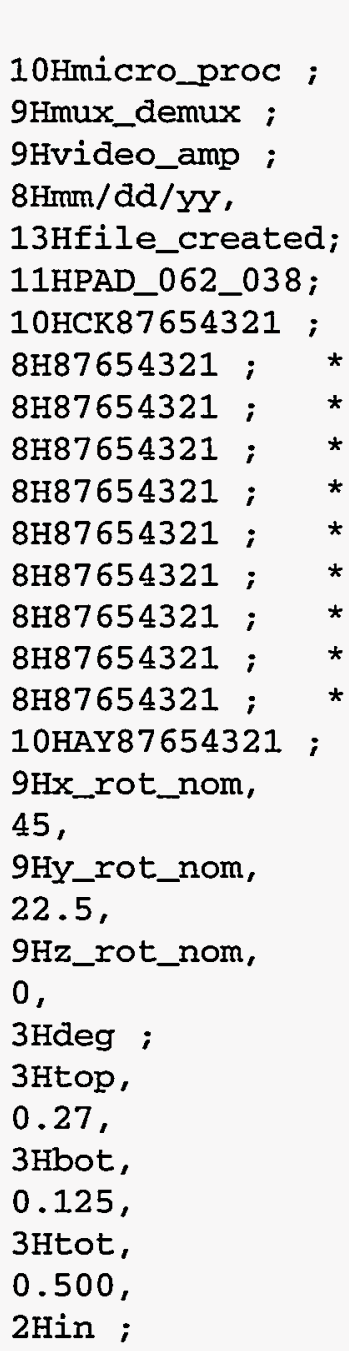 \\
\hline
\end{tabular}

* These fields are repeated as required. 
Table 6-8. Generic Properties of Network

\begin{tabular}{|c|l|l|l|l|}
\hline \multicolumn{5}{|c|}{ Parameter Data (fragment) } \\
\hline NP & \multicolumn{1}{|c|}{ Name } & NV & Type & Value \\
\hline \hline $\begin{array}{l}4, \\
1,\end{array}$ & $\begin{array}{l}\text { 16Hschematic_number, } \\
\text { 14Hstandalone_via; }\end{array}$ & 1, & 3, & 10HCK87654321; \\
\hline
\end{tabular}

Table 6-9. Generic Properties of Substrates

\begin{tabular}{|c|l|l|l|l|}
\hline \multicolumn{3}{|c|}{ Parameter Data (fragment) } \\
\hline NP & Name & NV & TY* & \multicolumn{1}{|c|}{ Value* } \\
\hline \hline 6, & 4Hdate, & 2, & 3, & 8Hmm/dd/Yy, \\
& & & 3, & 13 Hfile_creation \\
4, & 16Hschematic_number, & 1, & 3, & 10 HCK87654321; \\
4, & 10Hpwb_number, & 1, & 3, & 8 H87654321; \\
4, & 15Hassembly_number, & 1, & & 10 HCK87654321; \\
\hline
\end{tabular}

* These fields are repeated as required.

NOTE: Also see Generic Properties of Closed Curve.

Table 6-10. Properties of Component and/or LEP

\begin{tabular}{|c|l|l|l|l|}
\hline \multicolumn{3}{|c|}{ Parameter Data (fragment) } \\
\hline NP & Name & NV & Type & \multicolumn{1}{|c|}{ Value } \\
\hline \hline 4, & 15Habs_voltage_max, & 1, & 2, & $120.0 ;$ \\
4, & 9Htolerance, & 1, & 2, & $0.01 ;$ \\
4, & 10Htherm_cond, & 1, & 2, & $18.0 ; i$ \\
4, & 8Htherm_jc, & 1, & 2, & ww.www ; \\
4, & 7Htherm_r, & 1, & 2, & www.www ; \\
4, & 14Hjunction_max_t, & 1, & 2, & $125.0 ;$ \\
4, & 29Hphysical_component_device_tag, & 1, & 3, & $10 \mathrm{Hquad}$ _nand2 ; \\
4, & 30Helectrostatic_discharge_rating, & 1, & 3, & $3 \mathrm{H} 5 \mathrm{KV} ;$ \\
4, & 16Hcomponent_height, & 1, & 2, & $0.250 ;$ \\
4, & 26Hcomponent_outline_overhang, & 1, & 2, & $0.038 ;$ \\
\hline
\end{tabular}


Table 6-11. Generic Properties of Component Definitions (Type 308 or 320)

\begin{tabular}{|c|c|c|c|c|}
\hline \multicolumn{5}{|c|}{ Parameter Data (fragment) } \\
\hline NP & Name & NV & TYP* & Value* \\
\hline 4, & 18Hcomponent_std_name, & 1, & 3, & 11HSIC.0003.20; \\
\hline 4, & 17Hcomponent_version, & 1, & $3 ，$ & 7HVer1.03; \\
\hline 4, & 18Hcomponent_pkg_type, & 1, & 3, & 3Haxi; \\
\hline 4, & 19Hcomponent_tech_type, & 1, & 3, & 3Hsmt; \\
\hline 4, & 24Hcomponent_layout_surface, & 1, & 3, & 5Hfront; \\
\hline 6, & 26Hcomponent_insertion_origin, & 2, & 2, & $x \cdot \operatorname{xxx}$ \\
\hline 16, & 35Hcomponent_placement_feeder_location, & 7, & $\begin{array}{l}3, \\
2, \\
3, \\
2, \\
3, \\
2, \\
3,\end{array}$ & $\begin{array}{l}1 \mathrm{Hx}, \\
3.75, \\
1 \mathrm{Hy}, \\
5.25, \\
1 \mathrm{~Hz}, \\
2.5, \\
2 \mathrm{Hft}\end{array}$ \\
\hline 4, & 20Hcomponent_insertable, & 1, & 3 & 3Hyes; \\
\hline 4, & 16Hcomponent_height, & 1, & $2 ，$ & $0.25 ;$ \\
\hline 8, & 18Hcomponent_max_size, & 3, & $\begin{array}{l}2, \\
2,\end{array}$ & $\begin{array}{l}\mathrm{xxx} \cdot \mathrm{xxx}, \\
\mathrm{yYY} \cdot \mathrm{yYY}, \\
\mathrm{zzz} \cdot \mathrm{zzz} ;\end{array}$ \\
\hline 4, & 26Hcomponent_out1ine_overhang, & 1, & 2 ， & 0.038 \\
\hline
\end{tabular}

* These fields are repeated as required.

Table 6-12. Generic Properties of Component Instances (Type 408 or 420)

\begin{tabular}{|c|l|l|l|l|}
\hline \multicolumn{3}{|c|}{ Parameter Data (fragment) } \\
\hline NP & Name & NV & Type & \multicolumn{1}{|c|}{ Value } \\
\hline \hline 4, & 23Hcomponent_instance_side, & 1, & 3, & 3Htop; \\
4, & 18Hcomponent_preplace, & 3, & 2, & xx.xxx; \\
& & & 2, & yy.yyy \\
& 13Hcomponent_via; & & 3, & 3Htop; \\
4, & 14Hstandalone_via; & & & \\
& & & & \\
\hline
\end{tabular}


Table 6-13. Generic Properties of Connect Point (Type 132)

\begin{tabular}{|c|l|l|l|l|}
\hline \multicolumn{5}{|c|}{ Parameter Data (fragment) } \\
\hline NP & \multicolumn{1}{|c|}{ Name } & NV & Type & \multicolumn{1}{|c|}{ Value } \\
\hline \hline 1, & 20Hallowable_test_point; & & & \\
1, & 24Hnot_allowable_test_point; & & & \\
4, & 13Hpad_tech_type, & 1, & 3, & 3Hsmt; \\
4, & 26Hcomponent_default_padstack, & 1, & 3, & 11Hpad_062_038; \\
1, & 4Hpin1; & & & \\
\hline
\end{tabular}

Table 6-14. Generic Properties of Closed Curves

\begin{tabular}{|c|c|c|c|c|}
\hline \multicolumn{5}{|c|}{ Parameter Data (fragment) } \\
\hline NP & Name & NV & Type & Value \\
\hline $\begin{array}{l}1, \\
1, \\
4, \\
4, \\
4, \\
1, \\
4, \\
\star 4, \\
1, \\
1, \\
4, \\
8, \\
1, \\
4, \\
6,\end{array}$ & $\begin{array}{l}\text { 17Hplacement_outline; } \\
\text { 15Hthermal_outline; } \\
\text { 22Hcomponent_keep_outline, } \\
\text { 18Htrace_keep_outline, } \\
\text { 16Hvia_keep_outline, } \\
\text { 28Hphysical_padstack_definition; } \\
\text { 21Hplanar_shape_function, } \\
\text { ×25Hconductive_trace_function, } \\
\text { 28Hphysical_padstack_definition; } \\
\text { 26Hsubstrate_physical_outline ; } \\
\text { 23Hcomponent_instance_side, } \\
\text { 18Hcomponent_preplace, } \\
\text { 20Hlep_physical_outline; } \\
\text { 26Hcomponent_default_padstack, } \\
\text { 7Hfiducial, }\end{array}$ & $\begin{array}{l}1, \\
1, \\
1, \\
1, \\
* 1 \\
, \\
1, \\
3, \\
1, \\
2,\end{array}$ & $\begin{array}{l}3, \\
3, \\
3, \\
3, \\
* 3 \\
, \\
2, \\
2, \\
3, \\
3, \\
3, \\
3, \\
3,\end{array}$ & $\begin{array}{l}\text { 3Hout; } \\
\text { 3Hout; } \\
\text { 3Hout; } \\
\text { 3Hsignal; } \\
\text { *3Hsignal; } \\
\text { 3Htop ; } \\
\text { xx.xxx, } \\
\text { yy.yyY, } \\
\text { 3Htop; } \\
\text { 11HPAD_062_038; } \\
\text { 9Hcomponent, } \\
\text { 3Htop; }\end{array}$ \\
\hline
\end{tabular}


Table 6-15. Generic Properties of Open Curves

\begin{tabular}{|c|c|c|c|c|}
\hline \multicolumn{5}{|c|}{ Parameter Data (fragment) } \\
\hline NP & Name & NV & Type & Value \\
\hline $\begin{array}{l}4, \\
4, \\
1, \\
4, \\
1,\end{array}$ & $\begin{array}{l}\text { 25Hconductive_trace_function, } \\
\text { 21Hplanar_shape_function, } \\
\text { 9Htrace_bot; } \\
\text { 7Htrace_n, } \\
\text { 9Htrace_top; }\end{array}$ & $\begin{array}{l}1, \\
1, \\
1,\end{array}$ & $\begin{array}{l}3, \\
3, \\
1,\end{array}$ & $\begin{array}{l}\text { 8HFlowName; } \\
\text { 8HFlowName; } \\
4 ;\end{array}$ \\
\hline
\end{tabular}

Table 6-16. Generic Properties of Feature Definitions

\begin{tabular}{|c|ll|l|l|c|}
\hline \multicolumn{5}{|c|}{ Parameter Data (fragment) } \\
\hline NP & Name & NV & Type & Value \\
\hline \hline 6, & 8Hfiducial, & 2, & 3, & $\begin{array}{l}\text { (see**Iist), } \\
\text { (see*** list); }\end{array}$ \\
\hline
\end{tabular}

** Values: 9Hcomponent, 7Hcluster, 5Hboard, 5Hpanel.

*** Values: 3Htop, 6Hbottom.

Table 6-17. Generic Properties of Vias

\begin{tabular}{|c|l|c|c|c|}
\hline \multicolumn{3}{|c|}{ Parameter Data (fragment) } \\
\hline NP & \multicolumn{1}{|c|}{ Name } & NV & Type & Value \\
\hline \hline 1, & 13Hcomponent_via; & & & \\
1, & 15Hstand-alone_via; & & & \\
1, & 20Hallowable_test_point; \\
1, & 24Hnot_allowable_test_point; & & & \\
4, & 17Htest_point_pwb_id, & 16Hselected_test_point; & 3, & 5HTP321; \\
1, & 16Heled & & & \\
\hline
\end{tabular}


Table 6-18. Production Engineering Generic Properties

\begin{tabular}{|c|c|c|c|c|}
\hline \multicolumn{5}{|c|}{ Parameter Data (fragment) } \\
\hline NP & Name & NV & Type & Value \\
\hline 0 & 20Hpwb_physical_outline; & & & \\
\hline 2 , & 4Hdate, & 2, & $\begin{array}{l}3 \\
3\end{array}$ & $\begin{array}{l}\text { 8Hmm/dd/yy, } \\
\text { 13Hfile_creation; }\end{array}$ \\
\hline 4 & 26Hcomponent_default_padstack, & 1, & 3, & 11Hpad_062_038; \\
\hline 6 & 5Hpanel_offset, & 2 & 2 & $\mathrm{xx} \cdot \mathrm{xx}$ \\
\hline 4 & 14Hstation_number, & 1, & 1, & 420 \\
\hline 4 & 14Hstation_action, & 1, & 3, & 6 Hinsert; \\
\hline
\end{tabular}

Table 6-19. Insertion Machinery Generic Properties

\begin{tabular}{|c|c|c|c|c|}
\hline \multicolumn{5}{|c|}{ Parameter Data (fragment) } \\
\hline NP & Name & NV & Type & Value \\
\hline \hline 4, & 26Hcomponent_insertion_height, & 1, & 2, & $0.025 ;$ \\
\hline
\end{tabular}

Table 6-20. Placement Machinery Generic Properties

\begin{tabular}{|c|c|c|c|c|}
\hline \multicolumn{5}{|c|}{ Parameter Data (fragment) } \\
\hline NP & Name & NV & Type & Value \\
\hline $\begin{array}{l}4, \\
4, \\
4, \\
6, \\
4, \\
4, \\
8,\end{array}$ & $\begin{array}{l}\text { 33Hcomponent_placement_lead_diameter, } \\
\text { 22Hcomponent_placement_yn, } \\
\text { 25Hcomponent_placement_body, } \\
\text { 26Hcomponent_placement_center, } \\
\text { 29Hcomponent_placement_form_code, } \\
\text { 41Hcomponent_placement_form_code_descri } \\
\text { ption, } \\
\text { 25Hcomponent_insertion_force, } \\
\text { 28Hcomponent_physical_thickness, }\end{array}$ & $\begin{array}{l}1, \\
1, \\
1, \\
2, \\
1, \\
1, \\
3, \\
3,\end{array}$ & $\begin{array}{l}2, \\
3, \\
3, \\
2, \\
2, \\
1, \\
3, \\
2, \\
2, \\
2, \\
3, \\
2, \\
3,\end{array}$ & $\begin{array}{l}0.025 ; \\
1 \mathrm{Hn} ; \\
4 \mathrm{HTO} 22 ; \\
0.000 ; \\
0.000 ; \\
7 \text {; } \\
9 \text { Htombstone; } \\
37.54, \\
40.0 ; \\
47.5 ; \\
3 \text { Hmax, } \\
0.040 ; \\
2 \text { hin; }\end{array}$ \\
\hline
\end{tabular}


Table 6-21. Wire Wrap Application Properties

\begin{tabular}{|c|c|c|c|c|}
\hline \multicolumn{5}{|c|}{ Parameter Data (fragment) } \\
\hline NP & Name & NV & Type & Value \\
\hline $\begin{array}{l}1, \\
1, \\
1, \\
4, \\
4, \\
1, \\
4,\end{array}$ & $\begin{array}{l}\text { 27Htop_level_assembly_instance ; } \\
\text { 16Hlep_assembly_tag, } \\
\text { 9wire_wrap; } \\
\text { 5Hgauge, } \\
\text { 10Hwire_color, } \\
\text { 12Htwisted_pair; } \\
\text { 10Hwrap_class, }\end{array}$ & $\begin{array}{l}1, \\
1, \\
1 \\
1\end{array}$ & $\begin{array}{l}3, \\
1, \\
3, \\
3,\end{array}$ & $\begin{array}{l}\text { 12Hproto_wW_134 } \\
26 ; \\
4 \mathrm{Hblue} ; \\
1 \mathrm{Hb} ;\end{array}$ \\
\hline
\end{tabular}

Table 6-22. Production Test Generic Properties

\begin{tabular}{|c|l|l|l|l|}
\hline \multicolumn{5}{|c|}{ Parameter Data (fragment) } \\
\hline NP & \multicolumn{1}{|c|}{ Name } & NV & Type & \multicolumn{1}{c|}{ Value } \\
\hline \hline 1, & 20Hallowable_test_point; & & & \\
1, & 24Hnot_allowable_test_point; & & & \\
8, & 28Hcomponent_physical_thickness, & 3, & 3, & $2 \mathrm{Hmax}$ \\
& & & 2, & 0.040 \\
& & 2, & 3, & 2Hin; \\
6, & 23Hselected_test_point_tag, & 21 HDITMCO_9100, \\
4, & 18Htest_point_pwb_tag, & 1, & 3, & 8Hx312y207; \\
4, & 18Htest_point_nail_id, & 1, & 3, & 5Hpt329; \\
\hline
\end{tabular}

\subsubsection{LEP Entity Usage for Presentation (appearance)}

Presentation aspects of the graphics produced by IGES files are especially important for Engineering Drawing and Technical Illustration applications. Other documents (called out in CALS MIL-D-28000 Classes II and I) provide more detailed guidance for those applications. The principal focus of this AP has been to enable mechanical/electronic exchange of Layered Electrical Product data as simply and unambiguously as possible. 
Implementation \& Conformance Testing

BLANK PAGE 


\section{APPENDIX A. APPLICATION ACTIVITY MODEL}

\section{A. ACTIVITY MODEL CONTENTS}

\begin{tabular}{|c|c|}
\hline A-0 & Model Context \\
\hline A0 & Manage and Manufacture Products \\
\hline A0 Text & Text: Manage and Manufacture Products \\
\hline A2 & Perform Engineering \\
\hline A2 Text & Text: Perform Engineering \\
\hline A3 & Assure Product Quality \\
\hline A3 Text & Text: Assure Product Quality \\
\hline A32 & Perform Quality Appraisal \\
\hline A32 Text & Text: Preform Quality Appraisal \\
\hline A4 & Produce Products \\
\hline A4 Text & Text: Produce Productds \\
\hline A41 & Build Networks \\
\hline A41 Text & Text: Build Networks \\
\hline $\mathrm{A} 42$ & Assemble Devices \\
\hline A42 Text & Text: Assemble Devices \\
\hline A43 & Test Product \\
\hline A43 Text & Text: Test Product \\
\hline
\end{tabular}




\section{APPENDIX B}

\section{APPLICATION PROTOCOL USAGE GUIDE}

This section will include suggested algorithms for extracting various types of data from an IGES file which conforms to this AP. Ten processes have been identified as candidates for algorithms of which the first four are included in this AP version:

1. Drill-Hole / Cutouts

2. Netlists

3. BOM / LOM

4. Schematic Graphics

5. Physical Artwork

6. Automatic Insertion
7. Simulation

8. ATE Data

9. Automatic Inspection Data

10. Process Extractor

11. Wire Wrap

\section{ALGORITHM GUDELINES}

To effectively represent such extractions algorithms a form of "pseudo-code" is employed; much like that used in many of the computer science journals (e.g., Communications of the ACM). The format has few rules, allowing the algorithm writer considerable flexibility. The algorithms are included as an assist to the programmer and are not intended to present requirements for code.

\subsection{Algorithm Format}

Each algorithm will be called a "process" and will have the following major structure:

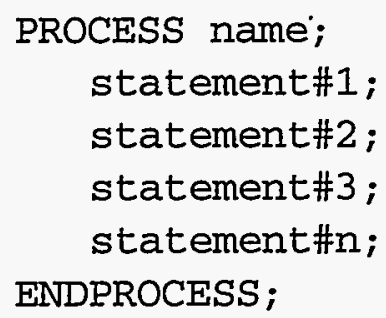

Within each process different statements will be defined to perform operations of iteration, flow control, and decision-making. Each statement will now be described in detail.

\subsubsection{STATEMENT GROUPING.}

A statement is defined to be a single line operation or group of single lines enclosed in a BEGIN/ END group. So whenever we refer to "statement" we either mean a one-line statement or a group of many lines. All statements should be terminated with a semicolon (;). Each line of the statement can be any english-like or programming-like description of an operation. Some examples include:

a. Single Statement Example (four single statements): get next record from IGES directory section; 
$i=i+1$

write out error message;

$j=k / 25.8$;

b. Group Statement Example:

BEGIN

get next record from IGES directory section;

$i=i+1$;

write out error message;

$j=k / 25.8$;

END;

\subsubsection{COMMENTS.}

An algorithm can contain comment lines. A comment line can appear anywhere in the algorithm and must be enclosed in a \{ \} pair as follows:

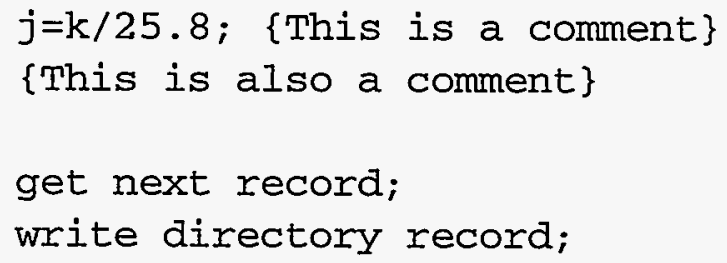

\subsubsection{IF/THEN/ELSE.}

IF some_condition THEN

statement\#1;

ELSE

statement\#2;

The following forms are also valid if each statement is in fact only one line:

IF some_condition THEN statement\#1;

ELSE statement\#2;

IF some_condition THEN statement\#1;

\subsubsection{LABELS.}

Any statement can have a symbolic label to be used as the address of a GOTO.

label : statement\#1;

statement\#2;

GOTO label;

\subsubsection{GOTO.}

The GOTO statement has the form:

\subsubsection{CASE.}

GOTO label;

A multi-way decision "CASE" statement has the following form:

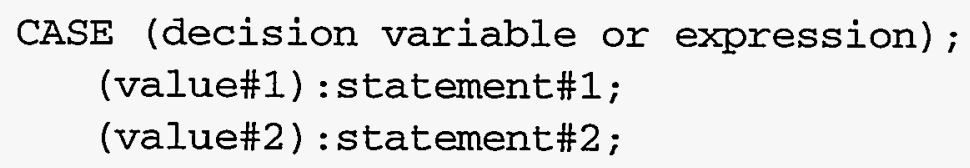




\section{DEFAULT : statement\#3; \\ ENDCASE;}

If the decision has a matching "value" then ONLY the set of statements for that value are executed. The DEFAULT is not required, but if present the default statements are executed if no values match.

\subsubsection{FOR.}

A for-loop construct is provided and it has the form:

FOR index_variable=start_value, end_condition statement\#1;

FOR index_variable+start_variable, end_condition BEGIN

statement\#1;

statement\#2;

statement\#3; END;

\subsubsection{WHILE.}

A while-loop construct is provided and it has the form:

WHILE (condition)

Do statement\#1;

\subsubsection{EXIT.}

The exit statement causes the algorithm to terminate immediately:

EXIT;

\subsection{Guidelines For Writing IGES Extract Algorithms}

When writing an extraction algorithm we suggest one follow a few guidelines to make the algorithm both easy to read and understand.

- Always indent nested constructs

- Try to keep each process short; one to three pages at the most.

- Keep the algorithms at a very abstract level. We assume reader is very familiar with the IGES format and has access to the specification for which the algorithm was written.

\section{IGES EXTRACTION ALGORITHMS}

\subsection{ALGORITHM : Drill Hole \\ IGES VERSION : 3.0}




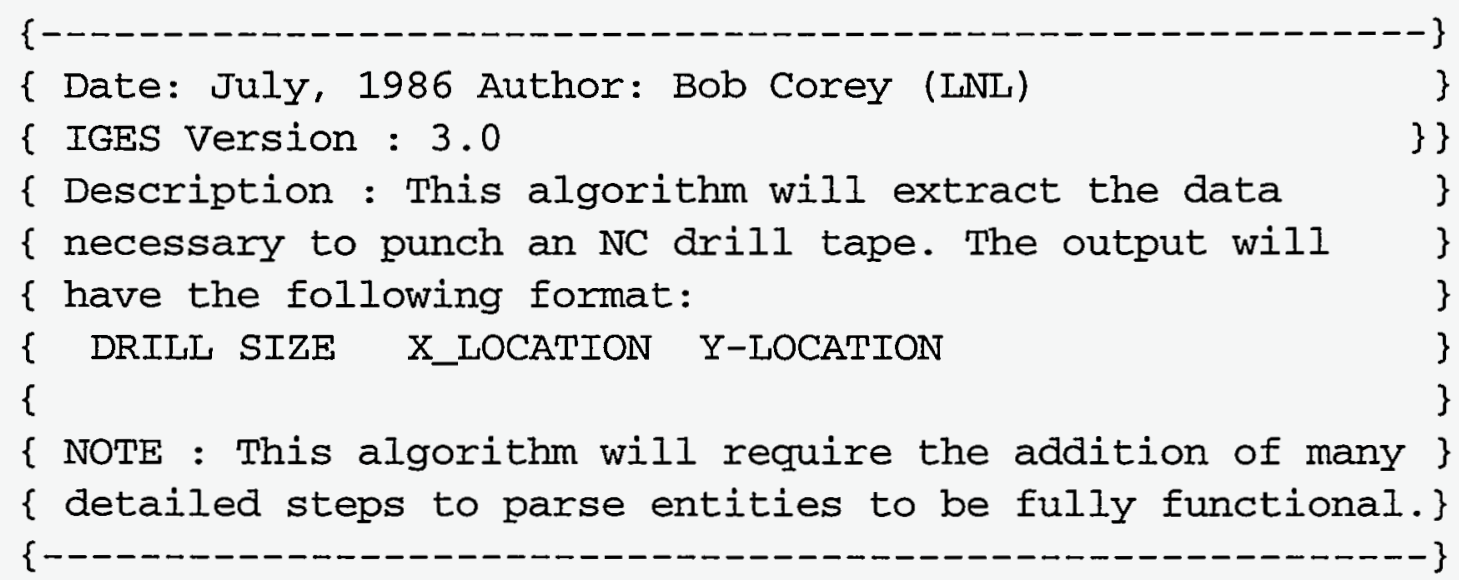

PROCESS Dril1_Hole;

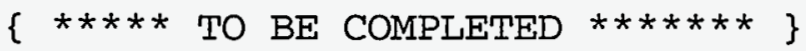

ENDPROCESS; \{Drill_Hole\}

\subsection{ALGORITHM : Netlist Extraction}

IGES Version : 3.0 and above

Purpose: Extract a signal netlist from a LEP AP IGES file.

Description: A netlist lists every signal on the PCB, and lists every connection point on each signal. Connection points include: component through hole pins, vias, and test points.

Information: Signal names, component reference designators, component pins, component pin names, vias, and test points.

$$
\begin{array}{ll}
\text { Reset } & \mathrm{U} 1-4, \mathrm{U} 11-4, \mathrm{U} 11-22, \mathrm{~J} 1-6 \\
\text { XCVI_123 } & \mathrm{R} 1-1, \mathrm{U} 11-6, \mathrm{~J} 1-7, \mathrm{TP} 123
\end{array}
$$

These lines show two different signals, "Reset" and "Xcvr_123". Each signalhas four connection points, one of which is a test point.

Note: input IGES file MUST be CALS Class III

Entities used

420 Network Subfigure Instance: the physical component on the PCB

132 Connect Point

402.18 Flow Associativity Property.

DE information: None of the DE information is used. 


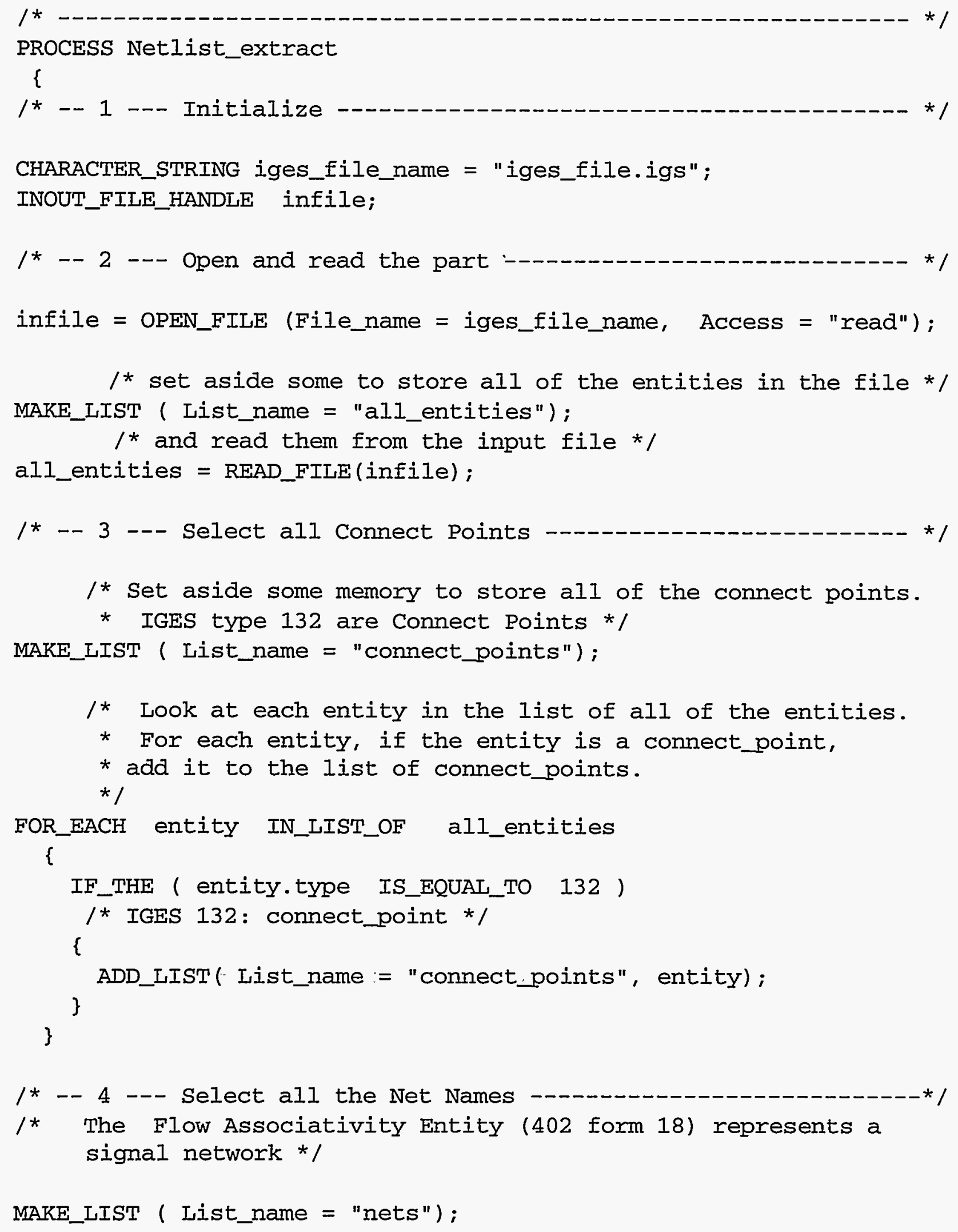




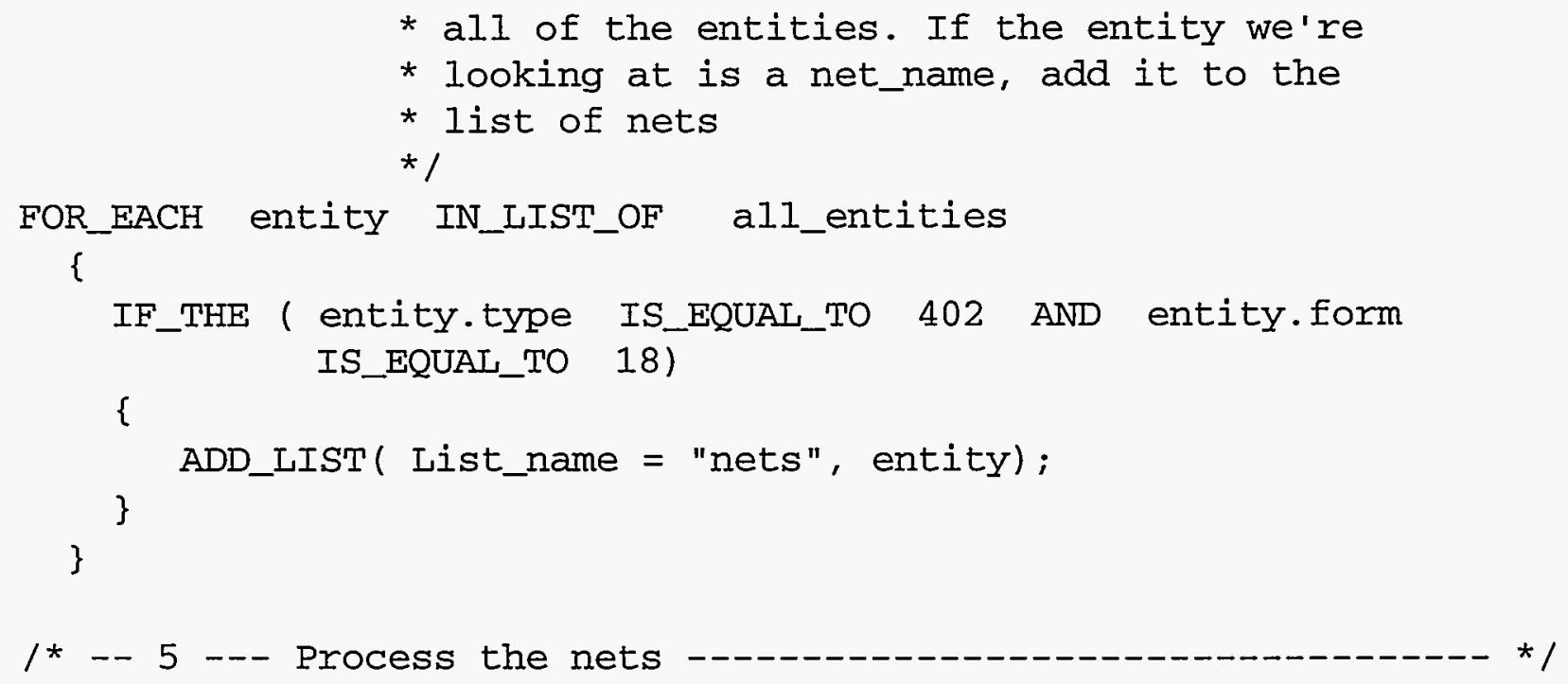




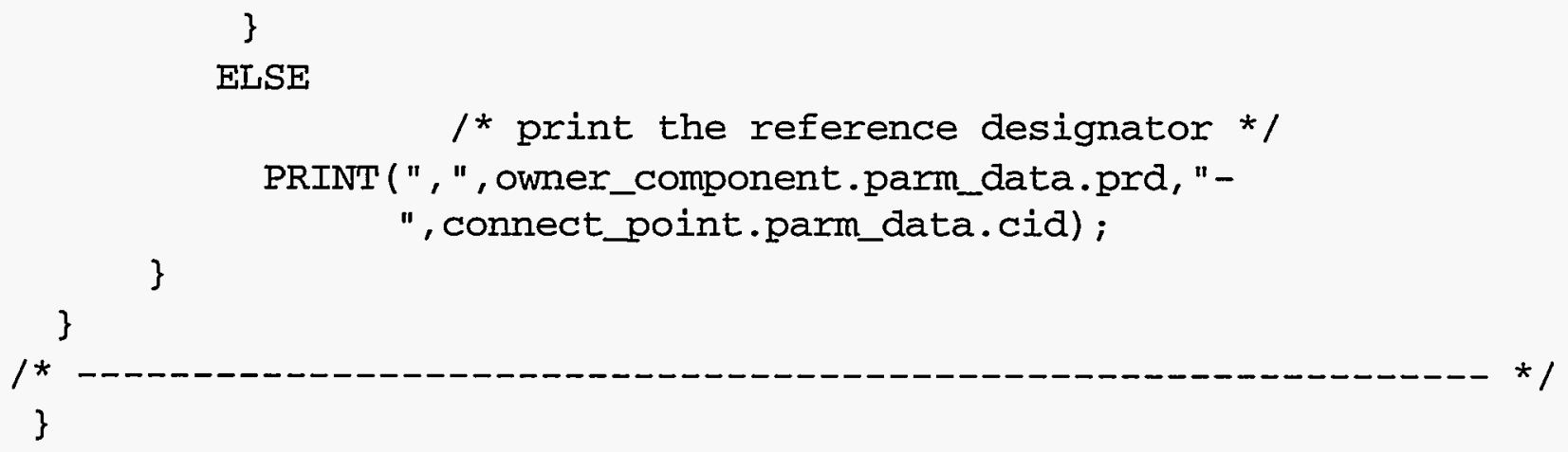

\subsection{ALGORITHM : Bill of Materials}

IGES VERSION : 3.0 and above

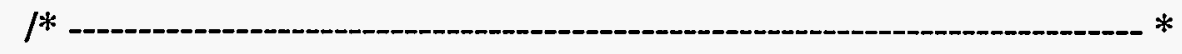

Algorithm for: Bill of Materials

Purpose: Extract a Bill of Materials (BOM) from a LEP AP IGES file.

Description: The bill of materials lists each part_type that is used on the board. For example, the following lines ae taken from a standard bom.

Information: Internal part number, description derived: count of parts for each part number. optional: component reference designators, item_number

ITEM_NUMBER COMPANY PART NO.COUNT DESCRIPTION REFERENCE

$13 \quad 2-60001-2360 \quad 1 \quad$ Resistor, $36 \quad$ R46

$14 \quad 2-60002-3383 \quad 12$ Resistor, $383 \quad$ R1 R6 R8 R9 R21

R22 R31 R36 R44

R45 R47 R48

15 2-60002-3590 8. Resistor, 590 R2 R7 R10 R11 R20

R23 R32 R37

These lines show that there are 3 part_types, all are resistors.

There will only be one part on the board of the part_type "Resistor, 36," (part_number "260001-2360"), and that will be R46. There will be 8 parts on the board of part_type "Resistor, 590" (part_number "2-60001-2360")

part_type: (aka call out) this is the identifyer for each type of part.

This can be thought of as a part number. 


\section{LEP AP Usage Guide}

(These are all of the Network Subfigure Defns 320)

component: This is the physical part stuck on the board.

(These are all of the Network Subfigure Instn 420)

Implementation notes.

Note: input IGES file MUST be CALS Class III

Entities used

320 Network Subfigure Definition: the "library" component

420 Network Subfigure Instance: the physical component on the PCB

406.9 Component part number

DE information: None of the DE information is used in the BOM.

Note: when storing the component definitions (320) and instances (420), any properties they reference must be stored with them. For example, each component instance must also store it's part number, it's description, and any other relevant information.

Geometric processing is not required.

Backpointer processing is not required.

Subfigure processing (reading, storing, and retreival) is not required.

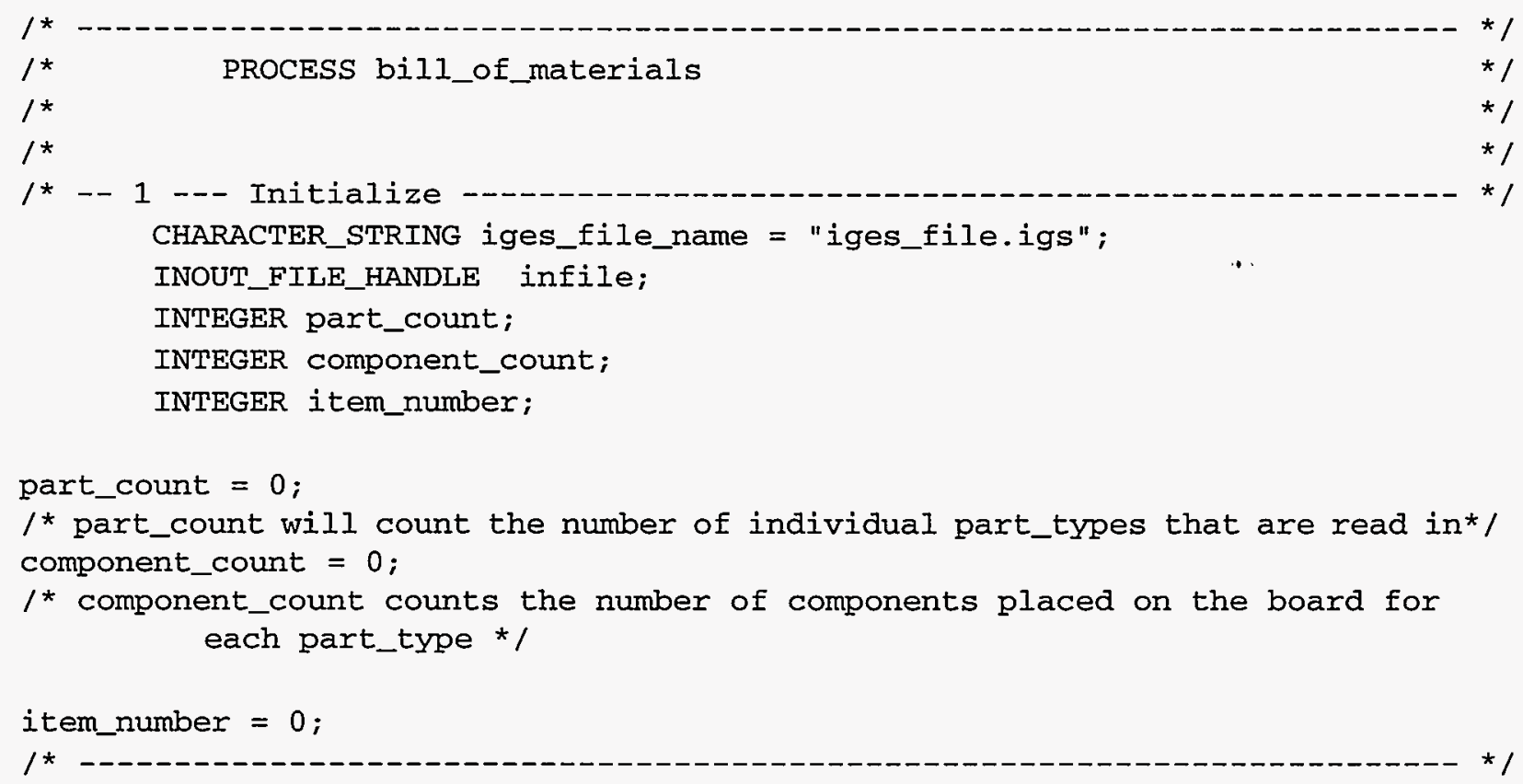




\section{LEP AP Usage Guide}

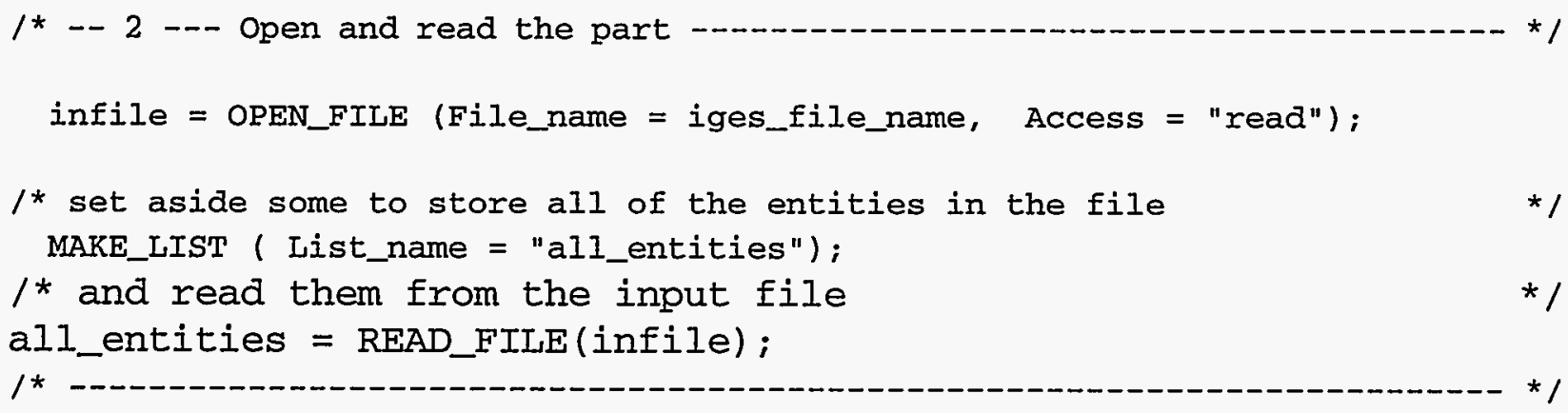



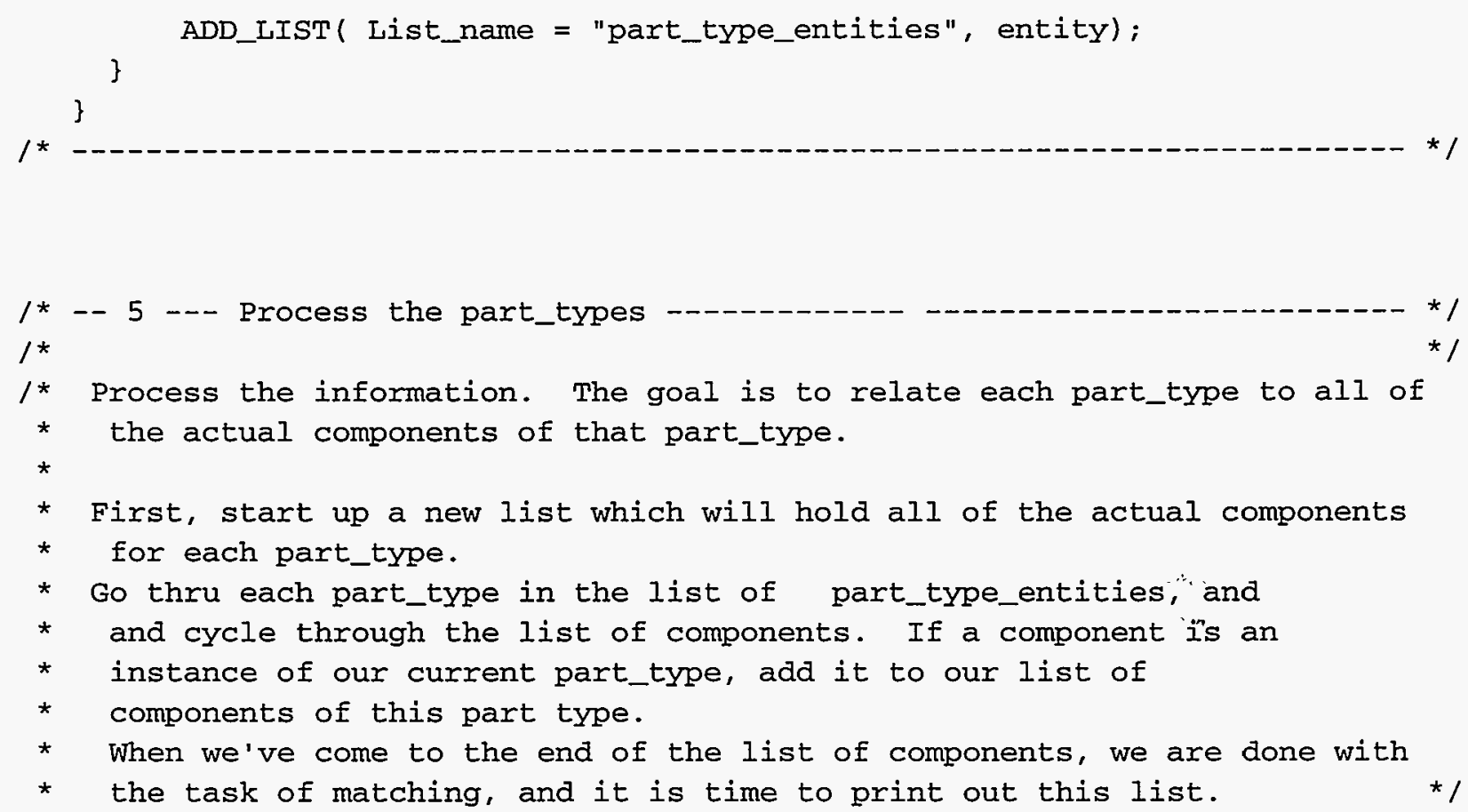


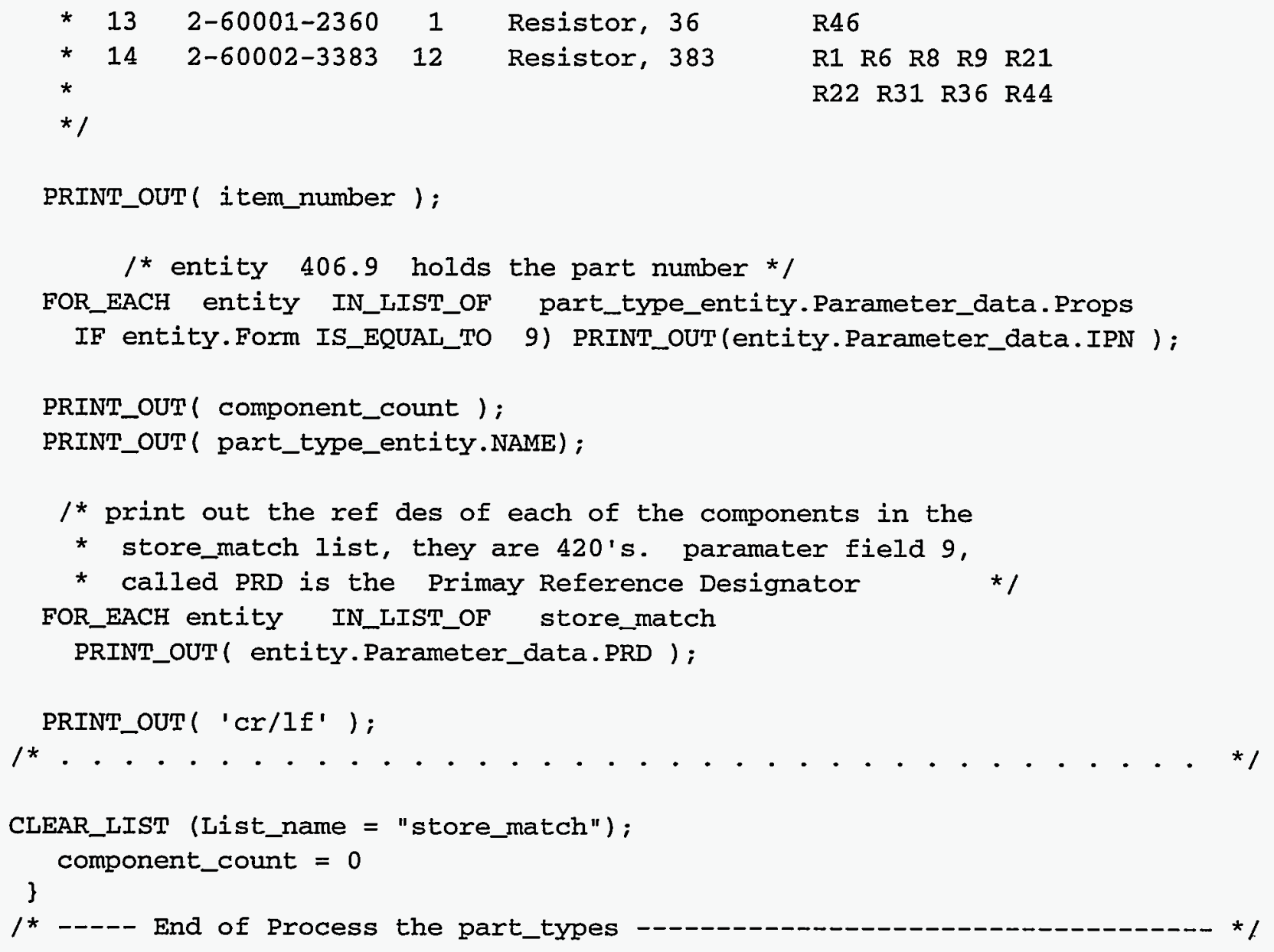

\subsection{ALGORITHM : Schematic (Graphics)}

IGES VERSION : 3.0

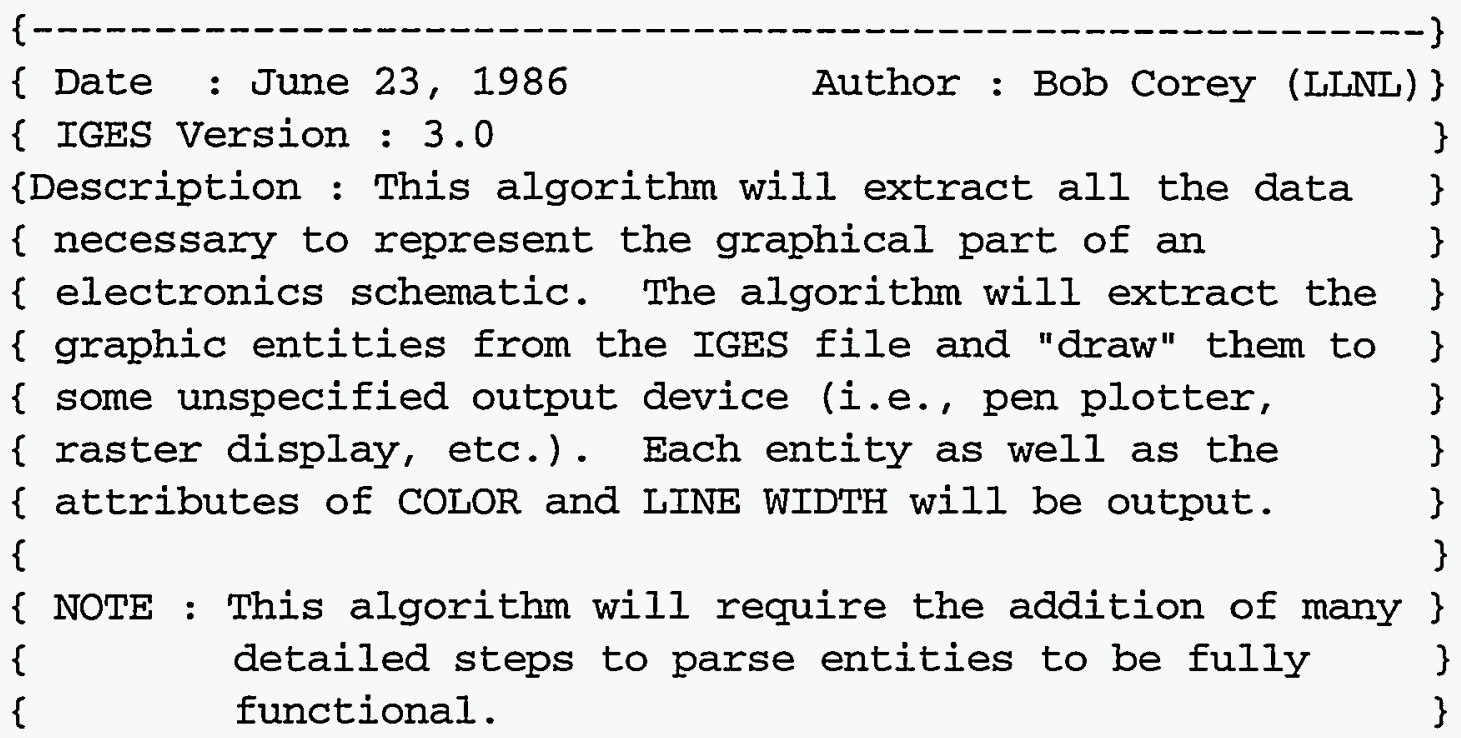




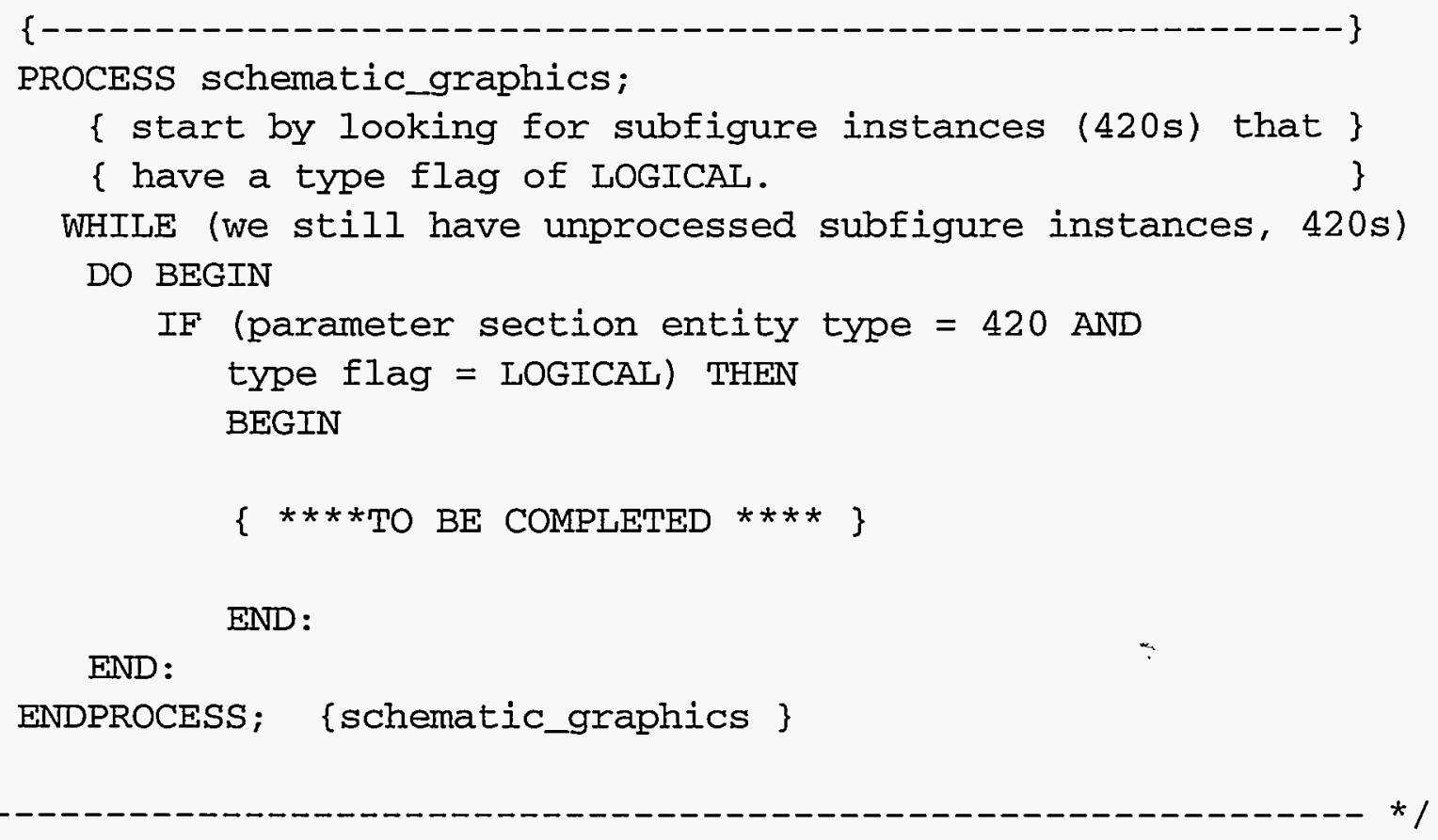

\subsection{Physical Artwork}

(***** UNDER CONSTRUCTION *****)

\subsection{Automatic Insertion;}

an Algorithm for: Pick/Place, and adhesive dispensing.

Purpose: Extract data that can be downloaded directly to surface mount component handling manufacturing equipment.

Description: Automated component handling manufacturing equipment such as pick and place may be driven using electronic data generated by CAE/CAD/CAM machines. This eliminates user entry error and drastically cuts time to market.

Information:

Required: component technology type, component part number, component reference designator, component placement side, component placement origin.

Derived: Component rotation is derived from a transformation matrix, if the component has one. Most component rotations will be orthogonal.

$\begin{array}{ccccc}\text { REF_DES } & X & Y & \text { Rotation } & \text { Part_Number } \\ \text { BAT1 } & 1.600 & 9.400 & 000 & \\ \text { U4 } & 0.100 & 0.600 & 000\end{array}$

Note: input IGES file MUST be CALS Class III 
Entities used:

320 Network Subfigure Definition

(Selection criteria)

420 Network Subfigure Instance (ref des and $x, y$ coords)

124 Transformation Matrix

406.9 Component part number

406.27 Component technology type (smt/thru_hole) *

406.27 Component placement side (top/bottom) *

*406.27 Component placement origin ( $x, y$ coords)

DE information: Transformation Matrix on Network Subfigure Instance.

Note: when storing the component definitions (320) and instances (420), any properties they reference must be stored with them. For example, each component instance must also store it's part number, it's description, and any other relevant information.

Note: The use of 406 form 27 entities reflect a defined and reccommended method of representing information

consistently. This is a new method, as of August 1994, and will not be found in files before this date. The pick/place algorithm is still valid, however, it is left to the user to define the method for defining technology, placement side, and origin.

Note: Component placement origin is used only if the placement origin is different than the the instance origin. For example, a resistor that is .5 inch long may be defined with pin 1 as it's origin, thus pin 2 is .5 inch away, and the center of the component is .25 inches from the origin. When the component is taken from the library and put on the board, pin 1 will be put the specified $x, y$ coordinate. However, the pick and place machine may define it's placement origin as being in the center of the part, thus the placement origin does not coincide with the instance origin.

*

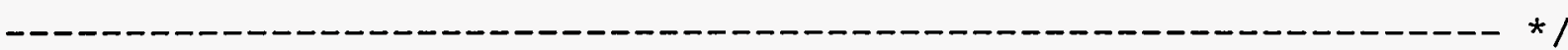

I* -

PROCESS pick_and_place

\{

The pick and place algorithm breaks down into a few simple steps. 


\section{LEP AP Usage Guide}

First, make a list of all of the appropriate part_types. They must be surface mount, and they must be on the side we are extracting data for. Second, make a list of all of the appropriate placed components. They too must be surface mount, and they must be on the side we are extracting data for.

Note: for demonstration purpose, we will assume we are looking for all surface mount components on the bottom side.

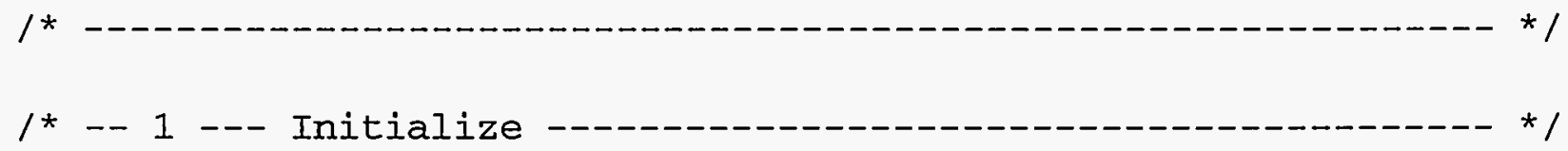

CHARACTER_STRING iges_file_name = "iges_file.igs" ;

INOUT_FILE_HANDLE infile;

INTEGER part_count;

INTEGER component_count;

INTEGER item_number;

part_count $=0 ; \quad I^{*}$ part_count will count the number of

* individual part_types that are read in */

component_count $=0_{i} / *$ component_count counts the number

* of components placed on the board for

* each part_type. */

item_number $=0$;

/* -------------

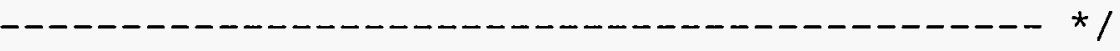

/* -- 2 -- Open and read the part

infile = OPEN_FILE (File_name = iges_file_name, Access = "read");

/* set aside some to store all of the entities

* in the file */

MAKE_LIST ( List_name = "all_ents");

1 * and read them from the input file */

al1_ents = READ_FILE (infile);

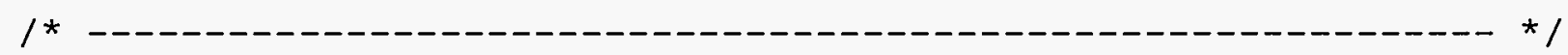

/* -- 3 --- Make a list of the appropriate part_types ---------- */

1 * set aside some memory to store all of the

* part_types IGES type 320 s network subfigure

* definition 
* 1

MAKE_LIST ( List_name = "part_type_entities");

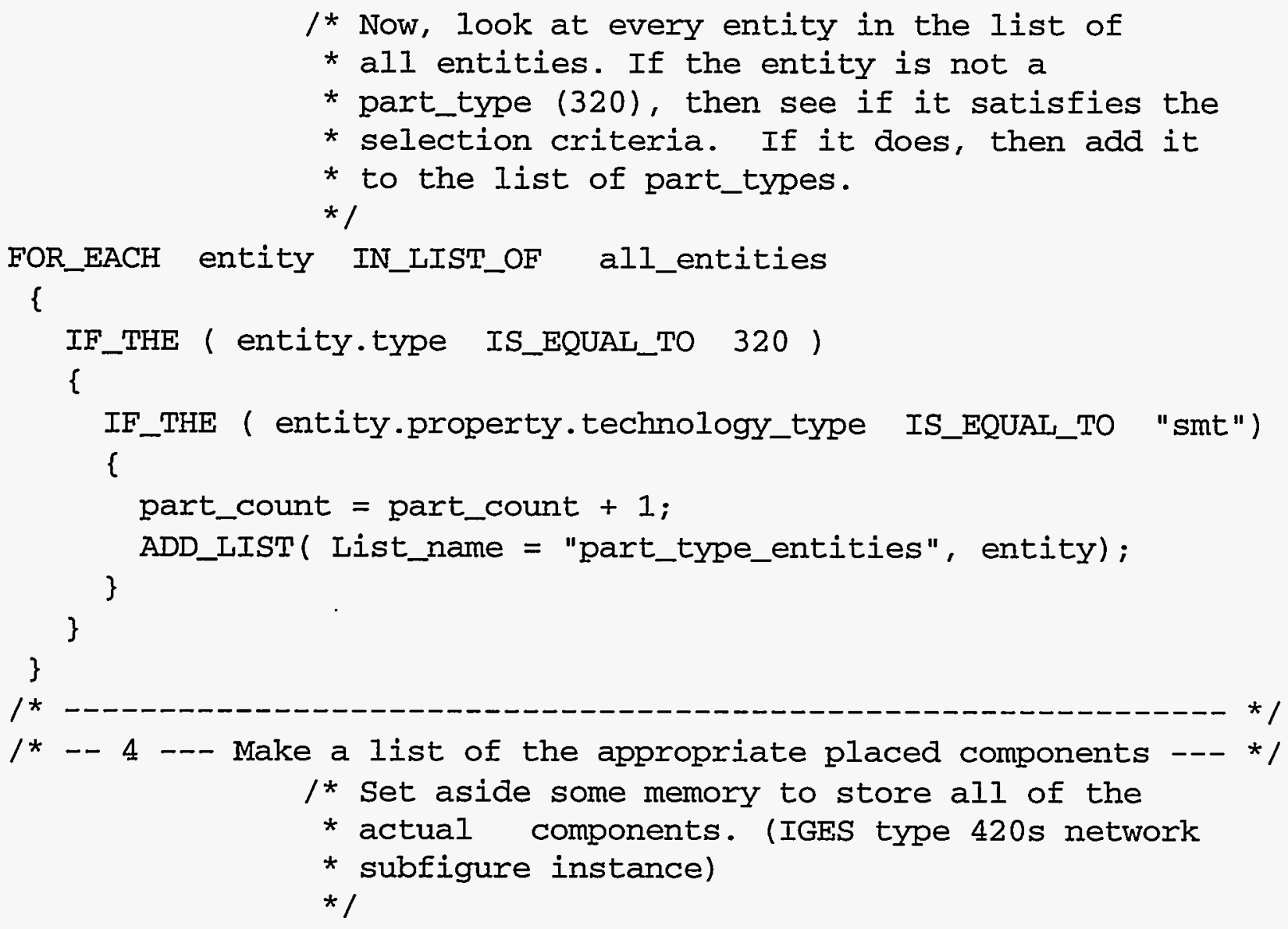




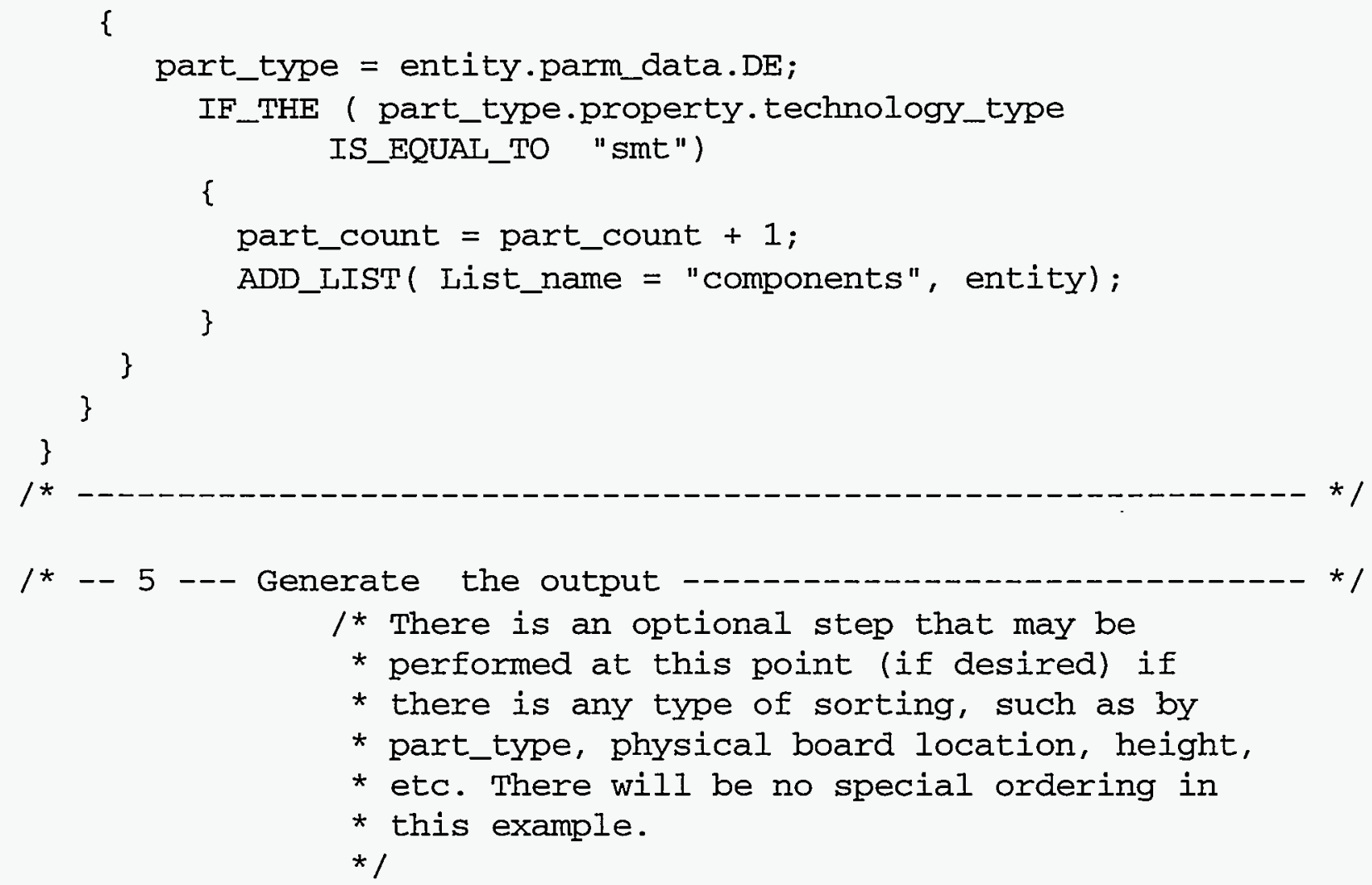




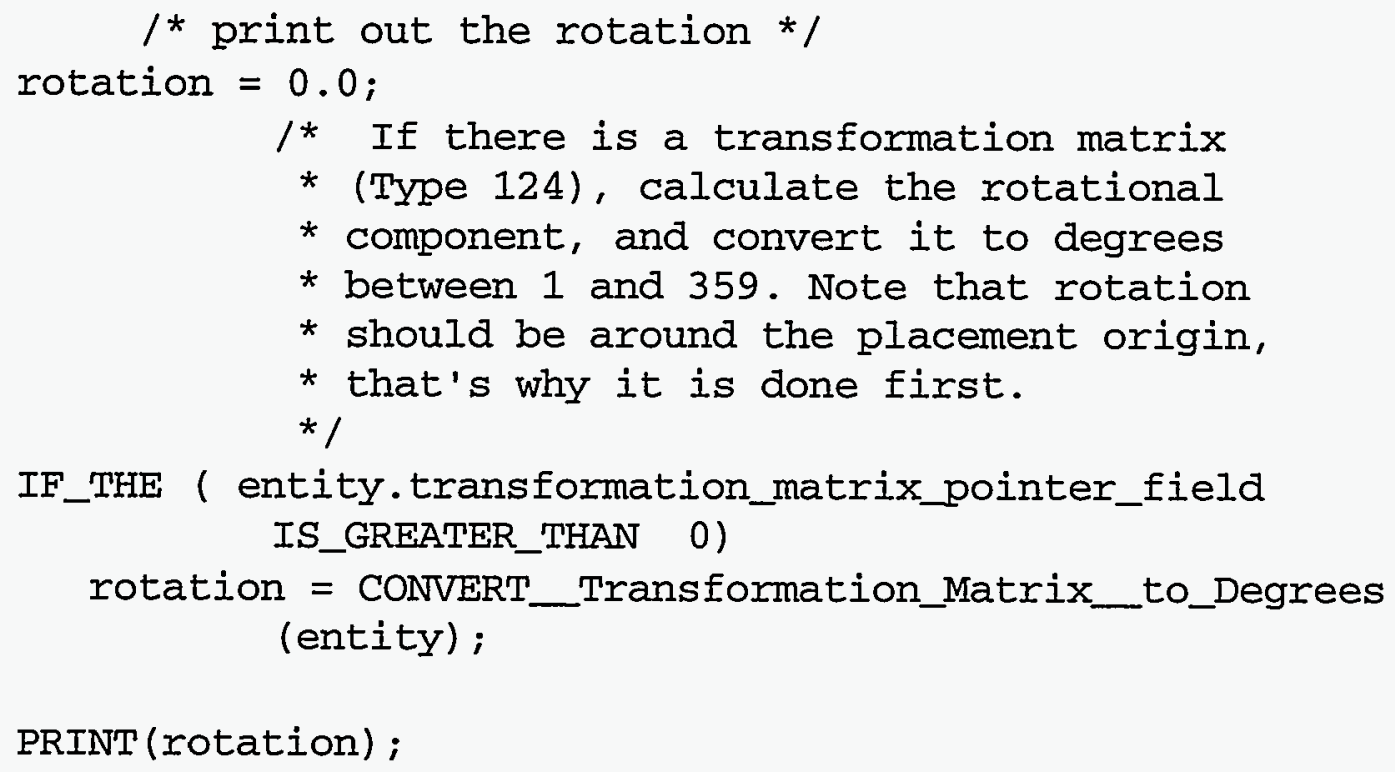

\subsection{Algorithm Simulation}

(***** UNDER CONSTRUCTION *****)

\subsection{Algorithm ATE Data}

(***** UNDER CONSTRUCTION *****)

\subsection{Algorithm Automatic Inspection Data}

(***** UNDER CONSTRUCTION *****)

\subsection{Algorithm Process Extractor}

(***** UNDER CONSTRUCTION *****)

\subsection{Algorithm Wire Wrap}

(***** UNDER CONSTRUCTION *****) 
LEP AP Usage Guide

\section{BLANK PAGE}

Page B- 18 


\section{APPENDIX C}

\section{TECHNICAL DISCUSSIONS}

\section{C.1. A Primary Parent for the ARM}

View 4-1 contains 7 independent entities, as opposed to most models which have a single independent entity. The multiple independent entity situation was recognized in the early STEP modeling, with one of these models proposing the Enterprise as the single independent parent. The MicroCIM team has discussed adding such an entity, however did not do so because the scope defined for the model does not require such a model refinement. It was recognized that each ARM independent entity employs "ID" in the key. ID is defined in the section 3 Glossary to include data which uniquely identifies the source or owning enterprise. Thus an Enterprise parent is implied.

\section{C.2. Netlist Information}

The Net entity on ARM View 4-3 has been recognized as derived information. Typically the Net consists of a Signal Name with the Reference Designators and Pin Numbers to be associated (i.e., connected) with that signal. The source of Net information is the functional design of the circuitry as presented by a schematic. The Schematic in any particular product design may have been "back-annotated" with net list information from the package design. The Schematic entity itself has not been decomposed into its fundamental entities in this version of the ARM.

\section{C.3. Multiple Planning for a Product}

The association of Process information with the Product model presents a problem when "re-planning" takes place. This would occur if the product is not completely processed to a single process definition (e.g., second sourcing or reprocurement). The team view was that the model provides a place for a particular user to record his planning information. Should the Product data be relocated, the site-specific planning would not be populated, and may be re-defined at the next cite.

\section{C.4. Producibility Feedback}

The use of "producibility" data in a concurrent engineering requires much more attention. As yet the team has discovered no commonly agreed to model of how things like "cost penalties for nonstandard designs" or "more easily manufactured trade-offs" are to be placed into a database which engineering could access during layout. These sorts of dispositions seem to take place during "producibility reviews" which requires the knowledge of experienced operations people. As a starting point for capturing such information, several kinds of Rules have been included in the ARM.

\section{C.5. Quality}

Much of the Application Activity Model (AAM) has been reworded and modified to reflect the use of Total Quality Management (TQM) and Quality Function Deployment (QFD) being practiced. The team expects new attributes will be required for some of the ARM entities. 